\title{
On linking the filter width to the boundary layer thickness in explicitly filtered large eddy simulations of wall bounded flows
}

\author{
by \\ Md. Mahfuz Sarwar \\ Centre for Environmental Safety and Risk Engineering (CESARE) \\ College of Engineering and Science \\ Victoria University
}

Submitted in total fulfilment of the requirements of the degree of Doctor of Philosophy

August 2016 


\section{Declarations}

"I, Md. Mahfuz Sarwar, declare that the PhD thesis entitled 'On linking the filter width to the boundary layer thickness in explicitly filtered large eddy simulations of wall bounded flows' is no more than 100,000 words in length including quotes and exclusive of tables, figures, appendices, bibliography, references and footnotes. This thesis contains no material that has been submitted previously, in whole or in part, for the award of any other academic degree or diploma. Except where otherwise indicated, this thesis is my own work".

Signature:

Date: 31-08-2016 


\section{Abstract}

Computational fluid dynamics (CFD)-based three-dimensional predictive fire models are increasingly used for predicting fire growth and spread. The models provide information to fire safety engineers for designing fire protection systems in buildings. Although currently limited, in a generation CFD-based models are expected to be used by emergency organisations to obtain similar information during bushfires under a wide range of topographies, climates and types of vegetation for their resource allocation planning. To increase their accuracy, most promising CFD-based models are embedded with pyrolysis sub-models along with large eddy simulation (LES) schemes to account for turbulence. The fundamental idea of LES is to resolve the large energy containing eddies, and use sub-grid scale (SGS) model to simulate the effect of energy dissipation by small scale eddies on large eddies.

Obtaining grid independent LES can be elusive. The filtering technique, along with the SGS model, plays an important role in grid sensitivity and accuracy of LES. It is known that grid sensitive simulations present a major problem with implicitly filtered LES and this can lead to poor grid convergence. In the last two decades, explicit filtering schemes have appeared as a viable option offer a promising solution, where the model (essentially the filter width) is maintained constant while the discretization error is reduced by refining the grid. Implicit filter tend not to separate discretization error from the modelling error because they do not separate the turbulence model from the grid refinement. This leads to grid sensitive results even at low Reynolds number for wall bounded flows. A growing body of literature is available in which it is argued that explicit schemes are more likely to provide grid independent solutions and reduce the numerical errors significantly compared to implicit schemes. In this study, a systematic approach 
for wall bounded flows is proposed to obtain grid independence by combining an explicit LES scheme with a damped Smagorinsky sub-grid model. The aim is to separate modelling error from numerical error and thereby to obtain a grid independent result more readily. In this approach, emphasis is given to the selection of the filter width as a function of a physical parameter, namely boundary layer thickness (BLT). An analysis of the energy spectrum ensures that grid converged solutions at fixed filter are consistent with LES principles by setting fixed filter width within the inertial sub-range and accurately capturing all energy containing eddies.

In this study, an open source implicitly filtered LES code, Fire Dynamics Simulator (FDS), is taken as the baseline source code. First, the elusiveness of the grid convergence with the baseline four implicitly filtered LES sub-models (standard Smagorinsky, dynamic Smagorinsky, Deardorff and Vremen) is demonstrated. Out of the four, standard Smagorinsky model is found to be most promising for a high Reynolds number $(R e)$ flow. Then, this LES sub-model is modified to implement the explicitly filtered LES. This modified model and approach is successfully applied to two benchmark cases at two different two different $R e_{\lambda}$. A low $R e_{\lambda}$ case is realized in a buoyancy driven turbulent flow in a differentially heated cavity, and a high $R e_{\lambda}$ flow occurs over a backward facing step. The proposed approach provides a possible alternative way of selecting the LES filter in the absence of DNS resolutions as references. This study provides guidelines on the selection of filter width to grid spacing ratio (ultimately BLT to grid spacing ratio) for the proposed numerical scheme for resolving turbulent flows. This study also shows that the performance of explicit LES with coarser resolution is better than the implicit LES with finer resolution to obtain accurate numerical solution. 


\section{Acknowledgements}

I would like to thank my supervisors, Associate Professor Khalid Moinuddin and Professor Graham Thorpe from Victoria University, and Dr Matthew Cleary from the University of Sydney, for their active support, guidance, inspiration and encouragement.

I would also like to thank:

Drs Randall McDermott and Kevin McGrattan from NIST, USA, Dr Jason Floyd from Hughes Associates, USA and Dr Ruddy Mell from the U.S. Forest Service for their active support in providing the technical information required on the FDS fire model through the FDS and Smokeview discussion group.

Dr Duncan Sutherland for his generous support, assistance and sharing of knowledge.

H. M. Iqbal Mahmud and Sk Md Kamal Uddin, post-graduate students at Victoria University and my colleagues, for their active support, motivation and valuable advice throughout this challenging research work.

The Director of Centre for Environmental Safety and Risk Engineering (CESARE), Professor Vasily Novozilov for his kind support in the final phase of my degree.

Bushfire Cooperative Research Centre (BCRC), Australia for their financial assistance and providing a scholarship, and Victoria University for waiving my tuition fees during the $\mathrm{PhD}$ candidature period. 
Syeda Lutfunnesa, my mother, Salma Sultana my sister, Sadek, Saeed, Mahmud, and Murad my brothers, Syed Shahidul Haque my uncle, and other friends and family members for their continuous support, motivation and inspiration during ups and downs of this long journey of research.

Most importantly, above all, Nazia Nawshin, my wife for her endless support, inspiration, cooperation and patience and my two beloved sons Rehan and Nashwan for their understanding as well as their love and affection.

Md. Mahfuz Sarwar

August 2016 


\section{Dedication}

This thesis is dedicated to the memory of my late father Mohammad Yunus Bhuiyan 


\section{Table of Contents}

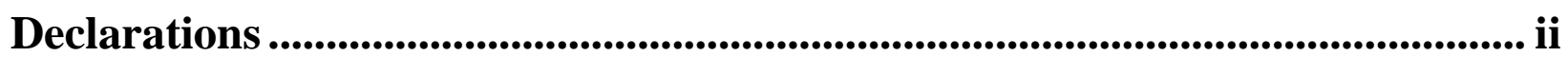

Abstract....................................................................................................................................... iii

Acknowledgements...................................................................................................................

Table of Contents ........................................................................................................... viii

List of figures................................................................................................................................. xii

List of tables.............................................................................................................................. xvi

Nomenclature ...................................................................................................................... xviii

1. Chapter 1 ........................................................................................................................1

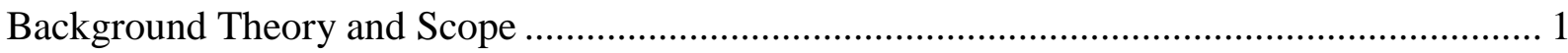

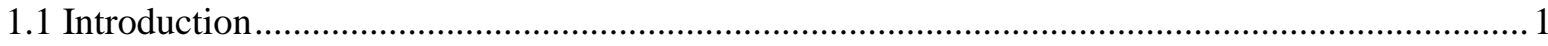

1.2 Numerical approaches to simulating turbulent flows..............................................................

1.2.1 Direct Numerical Simulation (DNS) ............................................................................

1.2.2 Reynolds Averaged Navier-Stokes Simulation (RANS) ………………………………….

1.2.3 Large Eddy Simulations (LES) ………………………………………………………6

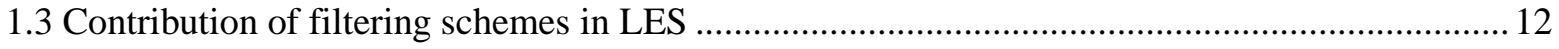

1.3.1 Implicit LES schemes ………………………………………………………………. 14

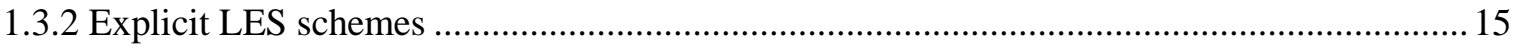

1.3.2.1 Selection of filter width in explicit LES scheme …………………………………...... 16

1.3.2.2 Filter to grid spacing ratio (FGR) in explicit schemes ................................................ 18

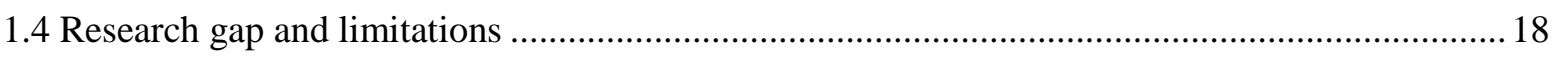

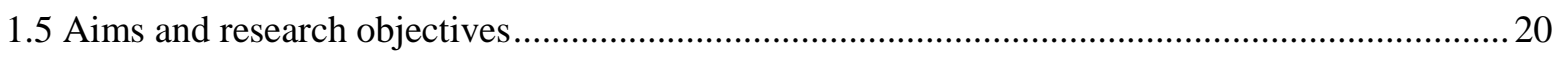

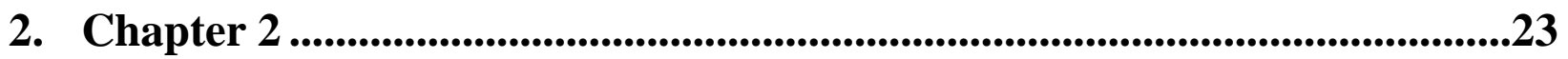

Governing equations and numerical schemes …………………………………………..... 23

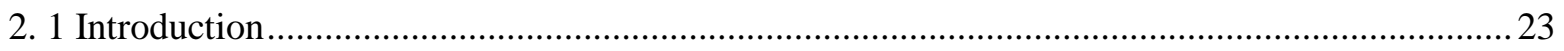

2.2 SGS modelling of turbulence in the baseline code ………….................................................2 


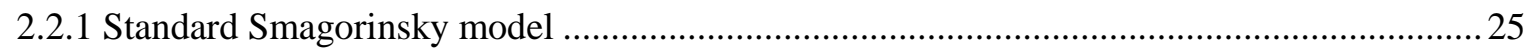

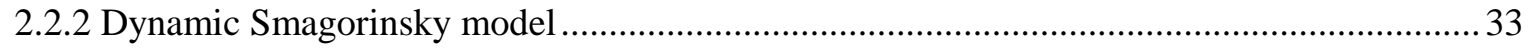

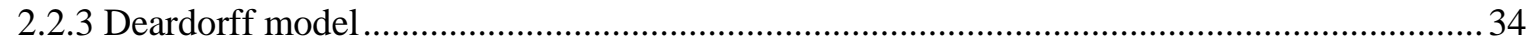

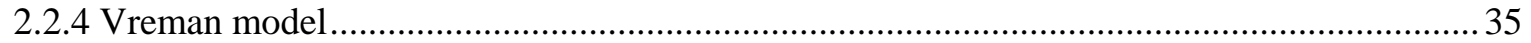

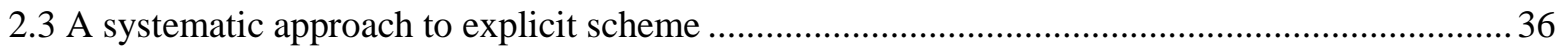

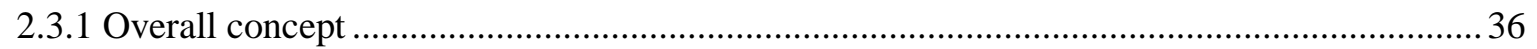

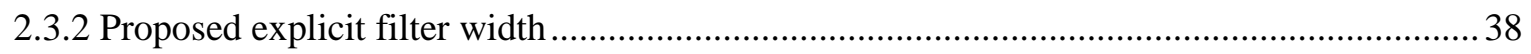

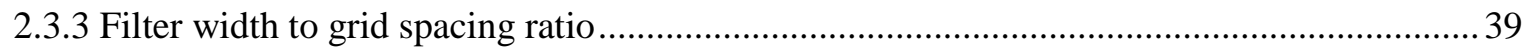

2.4 Governing equations of explicit LES scheme ........................................................................... 39

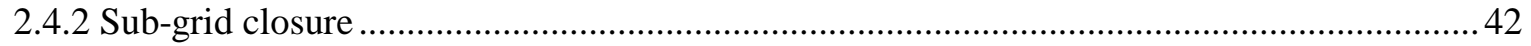

2.4.3 Damping function for model coefficient ............................................................................ 43

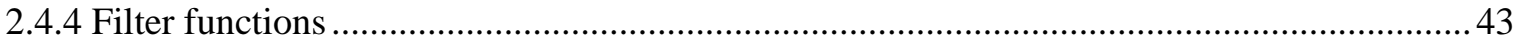

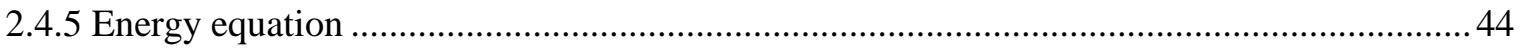

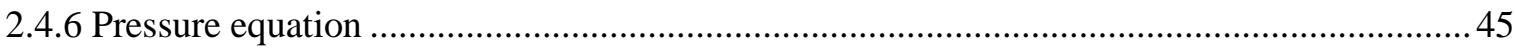

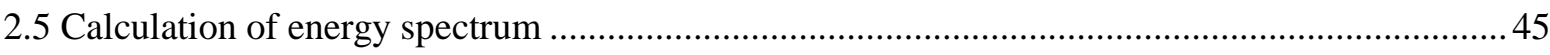

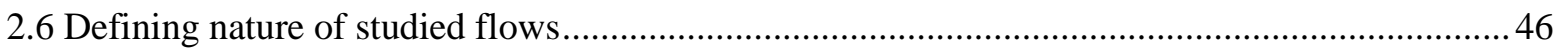

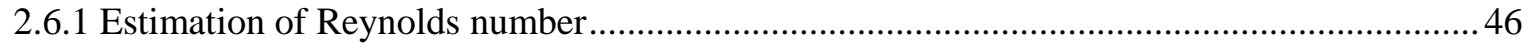

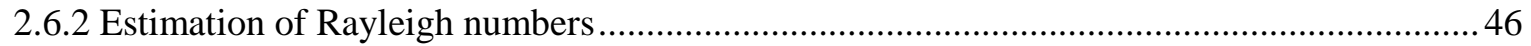

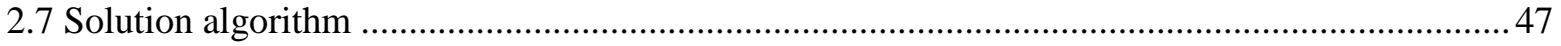

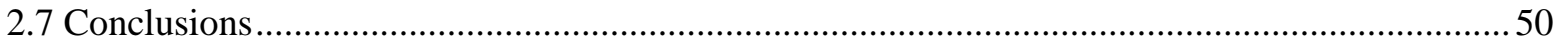

3. Chapter 3 .....................................................................................................................51

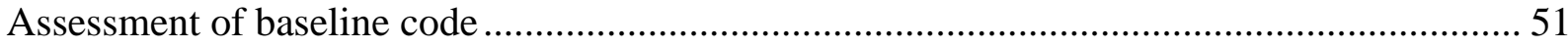

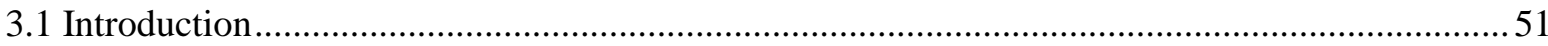

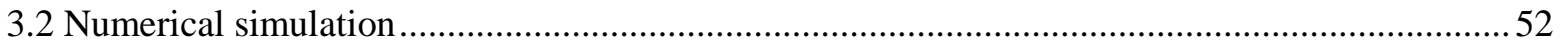

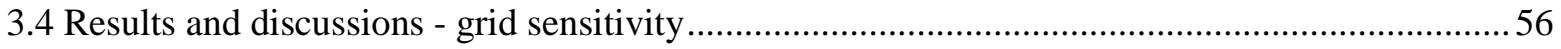

3.4.1 Graphical comparison -mean velocity and turbulence intensity .......................................57

3.4.2 Quantitative comparison - mean velocity and turbulence intensity ......................................59

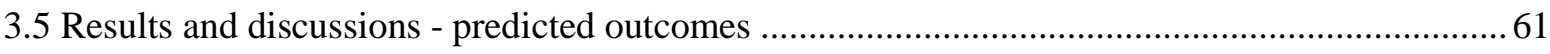

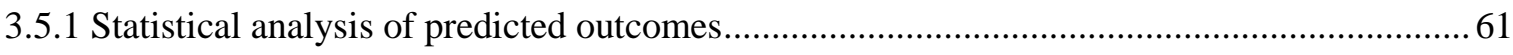

3.6 Further simulation with standard Smagorinsky model .......................................................6 65

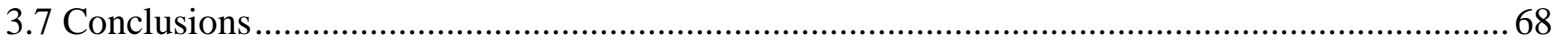

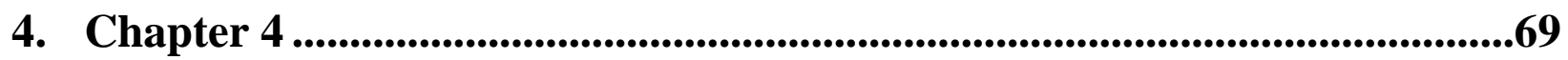




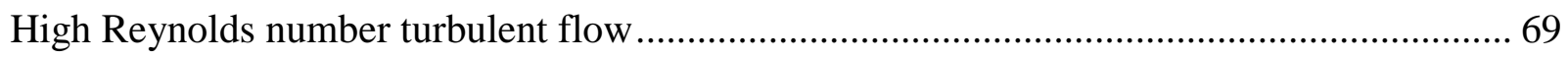

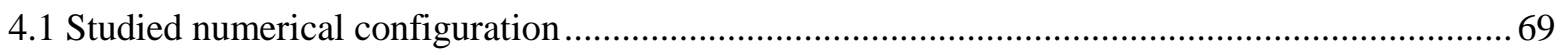

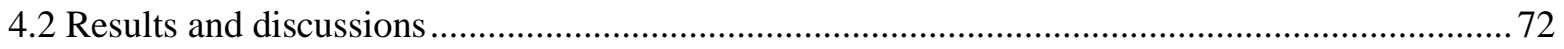

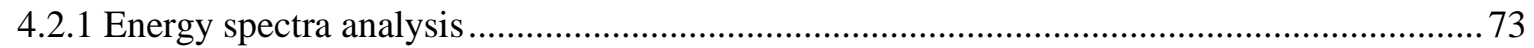

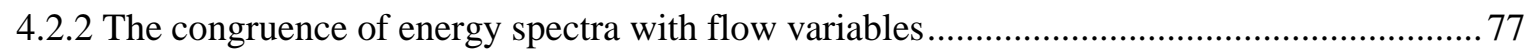

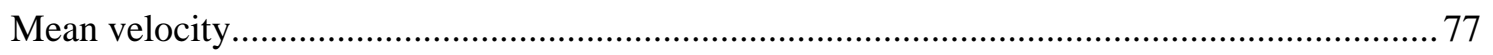

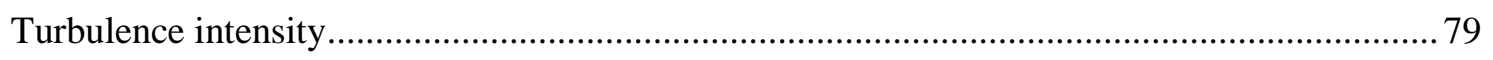

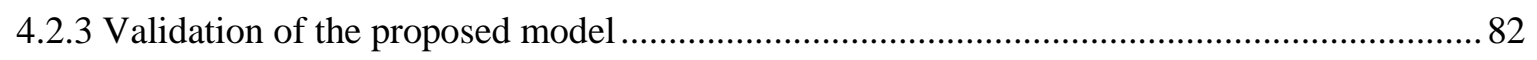

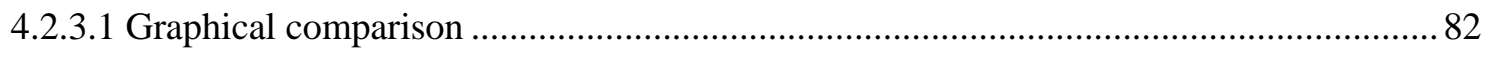

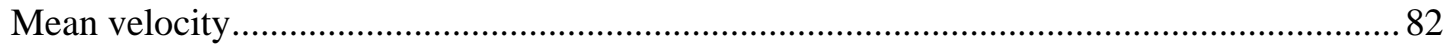

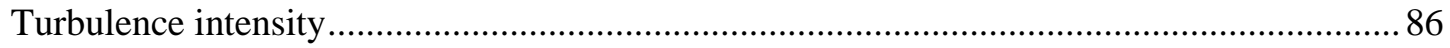

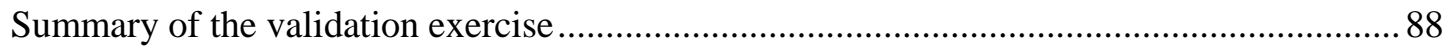

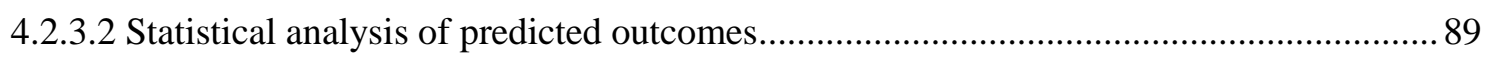

4.2.3.3 Comparison between simulation with implicit and explicit filtered LES ..................... 91

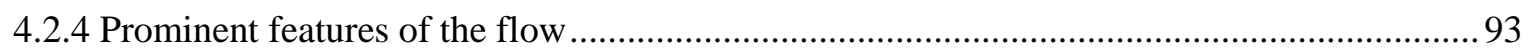

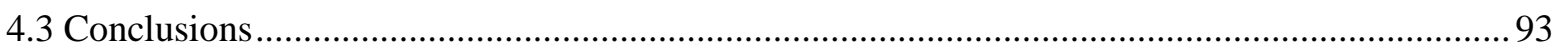

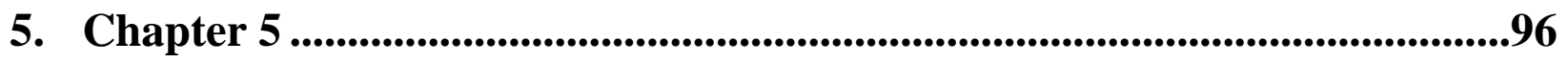

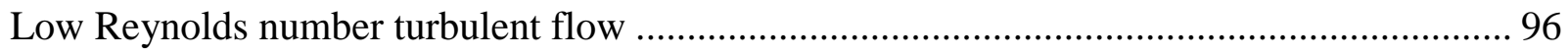

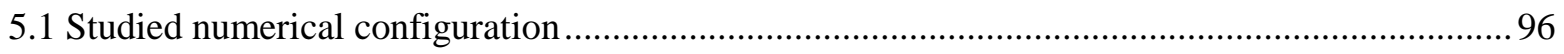

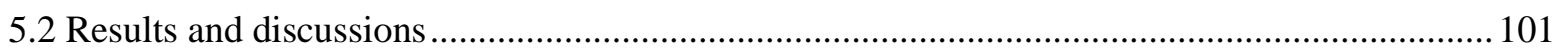

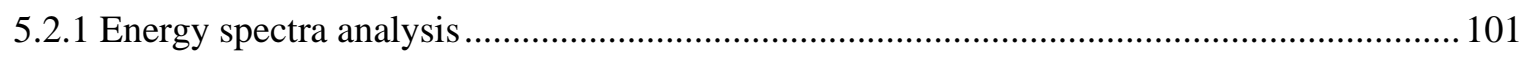

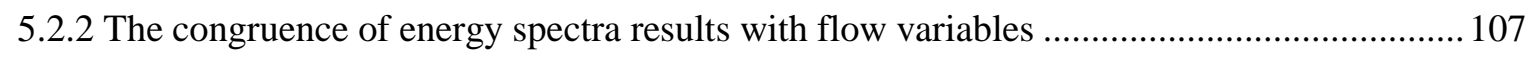

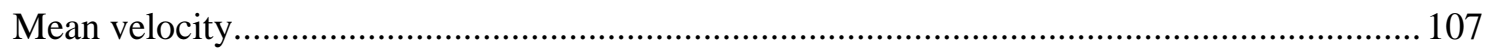

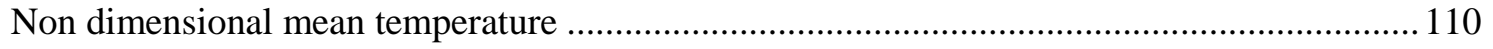

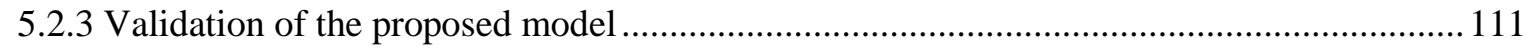

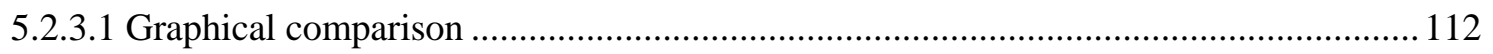

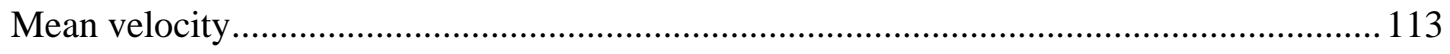

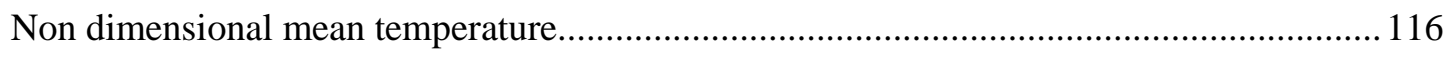

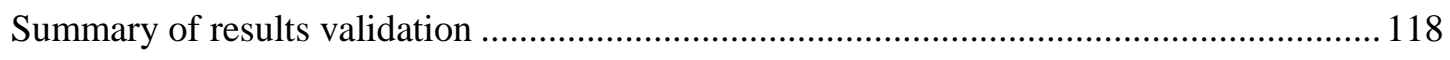

5.2.3.2 Statistical analysis of predicted outcomes.............................................................. 118

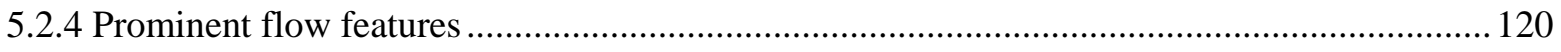

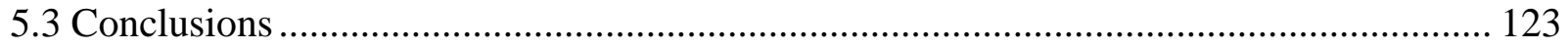




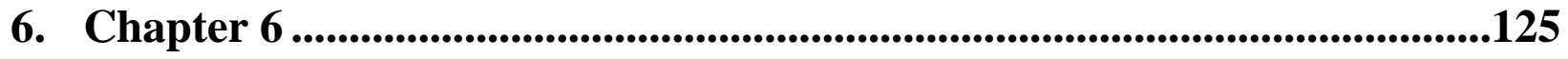

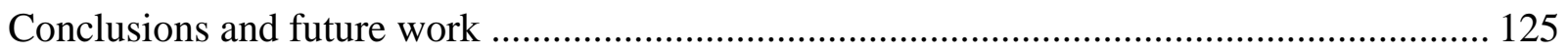

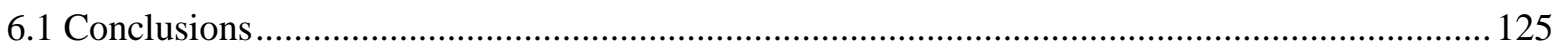

6.2 Future work and recommendations .................................................................................. 127

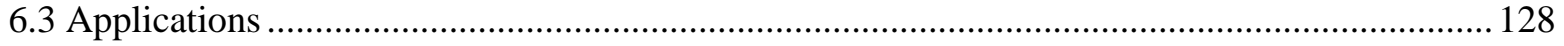

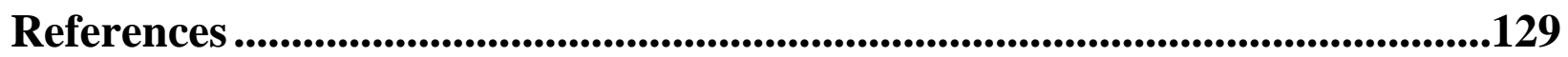

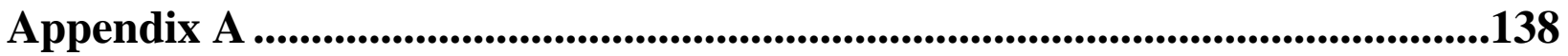

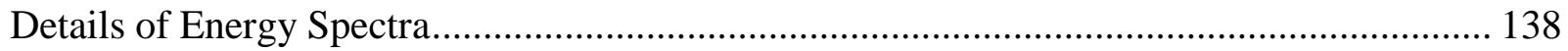

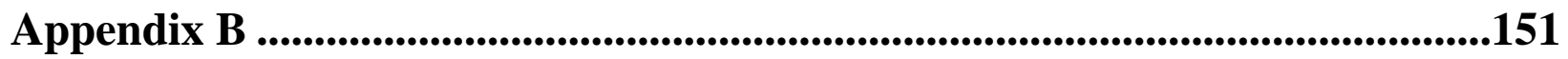

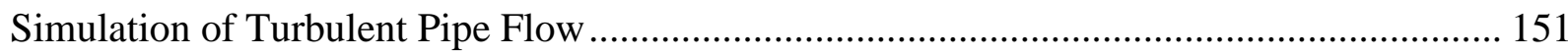

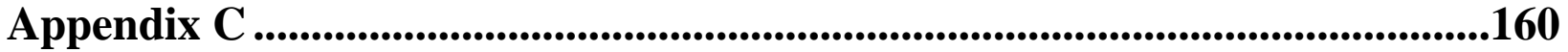

Relation between grid and $U e$.................................................................................... 160 


\section{List of figures}

Figure 1.1. Comparison of DNS, RANS and LES in terms of (a) computational costs and (b)

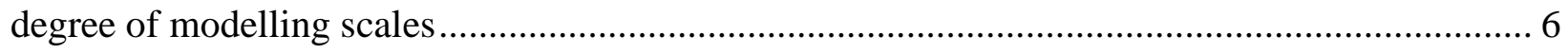

Figure 1.2. Representation of resolved and sub-grid scales (SGS)in LES(Sagaut 2001)............. 7

Figure 1.3. Energy cascading process from largest eddy scales to smallest eddy scales in terms of turbulent kinetic energy (vertical) and wavenumbers (inversely proportional to the length scales) plotted in log-log scales 13

Figure 2.1. Unfiltered and filtered turbulent field showing decomposition used in large eddy simulation. 24

Figure 2.2. Simulation of energy cascading process in DNS, LES and RANS. Vertical dashed line $\left(l_{E I}^{-1}\right)$ demarcates the inertial and the energy containing range and the dashed line $l_{D I}^{-1}$ demarcates the inertial and the dissipation range ............................................................ 26

Figure 2.3. Energy spectrum decomposition process in RANS .......................................... 27

Figure 2.4. Energy spectrum decomposition process in LES ......................................... 28

Figure 2.5. Filtered energy spectra for box and Gaussian filters ............................................. 29

Figure 2.6. Grid convergence of a hypothetical energy spectrum with progressively larger filter to grid ratios (FGR) $\Delta / \delta x_{1}<\Delta / \delta x_{2}<\Delta / \delta x_{3}$. The dashed vertical line indicates the cut-off wavenumber for the fixed filter width $\Delta$ 37

Figure 2.7. Hypothetical scenario of selection of filter width in an explicit scheme. Schematic shows the LES solution for four different filter widths with $\Delta_{1}>\Delta_{2}>\Delta_{3}>\Delta_{4}$ (all grid converged) and vertical lines are corresponding cut-off wavenumbers. 38

Figure 3.1. Schematic view of backward facing step flow configuration (not to scale)............. 52

Figure 3.2. Mean velocity profiles at test section $x / h=4$ for the SGS models ......................... 55 
Figure 3.3. Mean velocity profiles at test section $x / h=6$ for the SGS models 56

Figure 3.4. Turbulence intensities at test section $x / h=4$ for the SGS models 57

Figure 3.5. Turbulence intensities at test section $x / h=6$ for the SGS models 58

Figure 3.6. Comparison of the mean velocity profile at test sections $x / h=4$ and $x / h=6$ for the SGS models 63

Figure 3.7. Comparison of the turbulence intensity profile at test sections $x / h=4$ and $x / h=6$ for the SGS models. 64

Figure 3.8. Comparison of the mean velocity profile at test sections $x / h=4$ and $x / h=6$ for the standard Smagorinsky model for $h / \delta z=20$ 65

Figure 3.9. Comparison of the turbulence intensity profile at test sections $x / h=4$ and $x / h=6$ for the standard Smagorinsky model for $h / \delta z=20$ 66

Figure 4.1. Schematic diagram of grid used in Case 12 for a backward facing step ...... 72

Figure 4.2. Grid-convergence of three-dimensional energy spectra for the high Reynolds number flow with two different fixed filter widths. 73

Figure 4.3. Energy spectra analysis for cases in which the grid size is maintained constant and the filter width is varied. Case $1\left(\Delta_{1}=0.2 \delta\right)$ and Case $2\left(\Delta_{2}=0.1 \delta\right)$ 75

Figure 4.4. Mean velocity at four test sections for fixed filter Case $1\left(\Delta_{1}=0.2 \delta\right) \ldots \ldots \ldots \ldots \ldots \ldots \ldots . . . \ldots$

Figure 4.5. Mean velocity profiles at four test sections for fixed filter Case $2\left(\Delta_{2}=0.1 \delta\right) \ldots \ldots \ldots . .79$

Figure 4.6. Turbulence intensities at selected test sections for fixed filter Case $1\left(\Delta_{1}=0.2 \delta\right) \ldots . .80$

Figure 4.7. Turbulence intensities at selected test sections for fixed filter Case $2\left(\Delta_{2}=0.1 \delta\right) \ldots . .81$

Figure 4.8. Mean velocity grid converged solution at relatively coarser grid resolution for fixed filter Case $1\left(\Delta_{1}=0.2 \delta\right)$ and Case $2\left(\Delta_{2}=0.1 \delta\right)$ 83 
Figure 4.9. Mean velocity obtained with the finest grid resolution for fixed filter Case $1\left(\Delta_{1}=\right.$

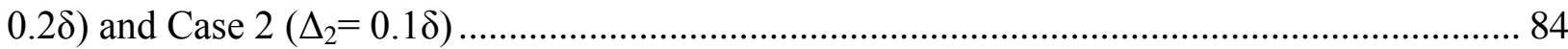

Figure 4.10. Mean velocity grid converged solution at same grid resolution for fixed filter

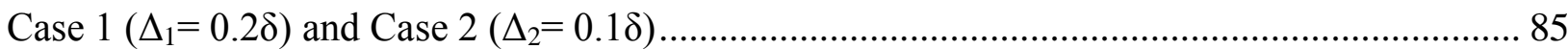

Figure 4.11. Grid converged solution of turbulence intensities at relatively coarse grid resolution

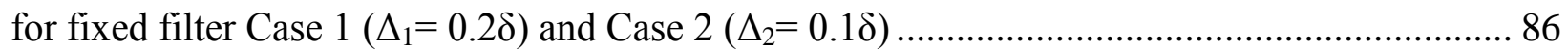
Figure 4.12. Turbulence intensities obtained with the finest grid resolution for fixed filter Case 1 $\left(\Delta_{1}=0.2 \delta\right)$ and Case $2\left(\Delta_{2}=0.1 \delta\right)$ 87

Figure 4.13. Grid converged solution of turbulence intensities at same grid resolution for fixed filter Case $1\left(\Delta_{1}=0.2 \delta\right)$ and Case $2\left(\Delta_{2}=0.1 \delta\right)$ 89

Figure 4.14. Instantaneous streamwise velocity at $12 \mathrm{~s}$ along the midsection of the high Reynolds number flow over a backward facing step. Results are for Case 23 93

Figure 5.1. Schematic of buoyancy driven flow inside a differentially heated rectangular cavity with particular emphasis is given to boundary layer development near the hot wall 97

Figure 5.2. Schematic diagram of grid resolutions for (a) Case 12 (b) Enlarged view near the top wall of rectangular cavity 100

Figure 5.3. Grid-convergence of three-dimensional energy spectra for the low Reynolds number flow with two different fixed filter widths 102

Figure 5.4. Energy spectra analysis with various grid and fixed filter combination for Case 1 $\left(\Delta_{1}=0.2 \delta\right)$ and Case $2\left(\Delta_{2}=0.1 \delta\right)$ 103

Figure 5.5. The model spectra at various Reynolds numbers (Pope 2000) 105

Figure 5.6. Mean velocity at four test sections for fixed filter Case $1\left(\Delta_{1}=0.2 \delta\right) \ldots \ldots \ldots \ldots \ldots \ldots . . \ldots 107$

Figure 5.7. Mean velocity at four test sections for fixed filter Case $2\left(\Delta_{2}=0.1 \delta\right) \ldots \ldots \ldots \ldots \ldots \ldots . . . \ldots 108$ 
Figure 5.8. Mean temperatures at four test sections for fixed filter Case $1\left(\Delta_{1}=0.2 \delta\right)$..... 109

Figure 5.9. Mean temperature at four test sections for fixed filter Case $2\left(\Delta_{2}=0.1 \delta\right)$. 110

Figure 5.10. Mean velocity grid converged solution at relatively coarser grid resolution for fixed filter Case $1\left(\Delta_{1}=0.2 \delta\right)$ and Case $2\left(\Delta_{2}=0.1 \delta\right)$ 112 Figure 5.11. Mean velocity obtained with the finest grid resolution for fixed filter Case $1\left(\Delta_{1}=\right.$ $0.2 \delta)$ and Case $2\left(\Delta_{2}=0.1 \delta\right)$ 113

Figure 5.12. Mean velocity grid converged solution at same grid resolution for fixed filter Case 1 $\left(\Delta_{1}=0.2 \delta\right)$ and Case $2\left(\Delta_{2}=0.1 \delta\right)$ 114

Figure 5.13. Mean temperature grid-converged solutions at relatively coarser grid resolutions for fixed filter Case $1\left(\Delta_{1}=0.2 \delta\right)$ and Case $2\left(\Delta_{2}=0.1 \delta\right)$ 115

Figure 5.14. Mean temperature obtained with the finest grid resolution for fixed filter Case 1 $\left(\Delta_{1}=0.2 \delta\right)$ and Case $2\left(\Delta_{2}=0.1 \delta\right)$ 116

Figure 5.15. Mean temperature grid converged solution at same grid resolution for fixed filter Case $1\left(\Delta_{1}=0.2 \delta\right)$ and Case $2\left(\Delta_{2}=0.1 \delta\right)$ 117

Figure 5.16. Snapshot of the shaded streamline plot (a) and vector plots (b) \& (c) of velocity inside the differentially heated cavity along the midsection at quasi-steady state after approximately $150 \mathrm{~s}$. Results are for Case 23.

Figure 5.17. A snapshot of the isotherms plots obtained after $2 \mathrm{~s}$ of initiating the experiment, (b) depicts the temperature distribution at the quasi steady state. (c) \& (d) that also indicate the quasi-steady state temperature and flow field on the mid-section of a differentially heated cavity. Results are for Case 23 after approximately 150s 122 


\section{List of tables}

Table 2.1. Smagorinsky constant $\left(C_{s}\right)$ for different types of filter.

32

Table 3.1. Considered model coefficients and estimated wall unit $\left(n^{+}\right)$values of SGS models .. 54

Table 3.2. Grid convergence measures (GCI) of the mean velocity profiles at stations $x / h=4$ and

$x / h=6$ for flow over a backward facing step

Table 3.3. Grid convergence measures (GCI) of the turbulence intensity at stations $x / h=4$ and

$x / h=6$ for flow over a backward facing step

Table 3.4. Relative error analysis of the mean velocity profile (fine resolution) at stations $x / h=4$

and $x / h=6$ for flow over a backward facing step

Table 3.5. Relative error analysis of the turbulence intensity (fine resolution) at stations $x / h=4$

and $x / h=6$ for flow over a backward facing step

Table 3.6. GCI of the mean velocity and the turbulence intensity profiles of standard

Smagorinsky model at stations $x / h=4$ and $x / h=6$ with very fine grid $(h / \delta z=20)$.

Table 3.7. Relative errors of the mean velocity and the turbulence intensity profiles of standard Smagorinsky model at stations $x / h=4$ and $x / h=6$ with very fine grid $(h / \delta z=20) \ldots \ldots \ldots \ldots \ldots \ldots \ldots . . . \ldots 7$

Table 4.1. Selected filter sizes $\Delta_{1}$ and $\Delta_{2}$ for a turbulent flow at high Reynolds numbers...... 70

Table 4.2. Grid resolutions for flow over a backward facing step at a high Reynolds number,

$R e_{\lambda}=115$

Table 4.3. Cross-stream averaged relative error of mean streamwise velocity in grid-converged

cases with different filter widths for flow over a backward facing step.

Table 4.4. Cross-stream averaged relative error of streamwise velocity variance in grid-

converged cases with different filter widths for flow over a backward facing step 
Table 4.5. Comparison of relative error of streamwise velocity and velocity variance between

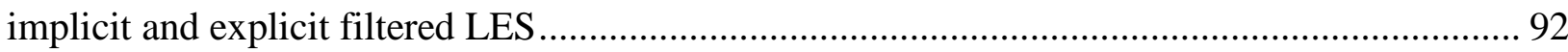

Table 5.1. Filter sizes $\Delta_{1}$ and $\Delta_{2}$ for a turbulent flow at low Reynolds numbers...................... 98

Table 5.2. Grid resolutions for a buoyancy driven differentially heated cavity flow at a low

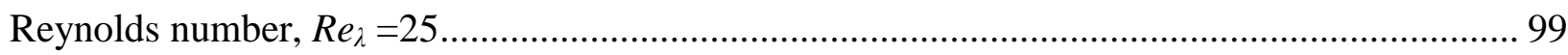

Table 5.3. Relative mean error analysis of mean velocity and dimensionless temperature for

Case 13 and Case 14 for cavity flow

Table 5.4. Relative mean error analysis of mean velocity and dimensionless temperature for

Case 23 and Case 24 for cavity flow 119 


\title{
Nomenclature
}

\author{
A integration constant in energy spectrum \\ $A^{+} \quad$ van Driest constant \\ B integration constant in velocity profile taken to be 5.2 \\ $B_{\beta} \quad$ expansion of velocity field using Taylor series in Vreman's model $\left(\mathrm{m}^{2} / \mathrm{s}^{2}\right)$ \\ C Kolmogorov constant \\ $c \quad$ Vreman's eddy model coefficient $\left(c=2.5 C_{S}\right)$ \\ $C_{\mu} \quad$ turbulent viscosity constant in the standard $k-\varepsilon$ model \\ $C_{\varepsilon 1}, C_{\varepsilon 2} \quad$ model constants in the standard $k-\varepsilon$ model \\ $C_{S} \quad$ Standard Smagorinsky model coefficient \\ $C_{S_{-} \text {Damp }} \quad$ Smagorinsky coefficient $\left(C_{S}\right)$ using the Van Driest damping function \\ $C_{i j} \quad$ cross-stresses $\left(\mathrm{N} / \mathrm{m}^{2}\right)$ \\ $C_{v} \quad$ Deardorff model coefficient \\ $E(\kappa) \quad$ energy spectrum $\left(\mathrm{m}^{3} / \mathrm{s}^{2}\right)$ \\ $\widehat{E}(\kappa) \quad$ filtered energy $\operatorname{spectrum}\left(\mathrm{m}^{3} / \mathrm{s}^{2}\right)$ \\ $E_{f} \quad$ filtered kinetic energy from the filtered velocity field $\left(\mathrm{m}^{3} / \mathrm{s}^{2}\right)$ \\ $e_{i j} \quad$ dissipation tensor $\left(\mathrm{m}^{2} / \mathrm{s}^{3}\right)$ \\ $F_{i} \quad$ external force $\left(\mathrm{N} / \mathrm{m}^{2}\right)$ \\ $F_{\text {Kleb }} \quad$ Klebanoff intermittency function \\ $f_{l}(\kappa l) \quad$ function of wavenumbers based on integral lengthscale \\ $f_{\eta}(\kappa \eta) \quad$ function of wavenumbers normalized by Kolmogorov lengthscale $(\eta)$
}


$G(r) \quad$ filter function in LES

$\widehat{G}(\kappa) \quad$ filter transfer function in LES

$G r_{x} \quad$ Grashofs number

$g \quad$ acceleration due to gravity $\left(9.81 \mathrm{~m} / \mathrm{s}^{2}\right)$

$H(x) \quad$ Heaviside function

$H \quad$ cavity height (m)

$\kappa \quad$ von Karman constant

$\kappa \quad$ wavenumbers $\left(\mathrm{m}^{-1}\right)$

$\kappa_{E I} \quad$ demarcation wave number between energy containing $\left(\kappa>\kappa_{E I}\right)$ and inertial range $\left(\kappa<\kappa_{E I}\right)\left(\mathrm{m}^{-1}\right)$

$\kappa_{D I} \quad$ demarcation wave number between inertial $\left(\kappa>\kappa_{D I}\right)$ and dissipation range $\left(\kappa<\kappa_{D I}\right)\left(\mathrm{m}^{-1}\right)$

$k \quad$ turbulent kinetic energy $\left(\mathrm{m}^{2} / \mathrm{s}^{2}\right)$

$k_{s g s} \quad$ turbulent kinetic energy of SGS model $\left(\mathrm{m}^{2} / \mathrm{s}^{2}\right)$

$k_{r} \quad$ turbulent kinetic energy for the residual part in LES $\left(\mathrm{m}^{2} / \mathrm{s}^{2}\right)$

$L \quad$ width of cavity (m)

l characteristic length scale $(\mathrm{m})$

$l_{0} \quad$ integral length scale $(\mathrm{m})$

$l_{E I} \quad$ demarcation between energy containing $\left(l>l_{E I}\right)$ and inertial range $\left(l<l_{E I}\right)$

$l_{D I} \quad$ demarcation between inertial $\left(l>l_{D I}\right)$ and dissipation range $\left(l<l_{D I}\right)$

$l_{\text {mix }} \quad$ mixing length $(\mathrm{m})$

$l_{s} \quad$ Smagorinsky length scale analogous to mixing length $\left(C_{s} \Delta\right)(\mathrm{m})$ 
$M_{i j} \quad$ model parameter in dynamic Smagorinsky model

$N_{x}, N_{y}, N_{z} \quad$ number of grid points in three spatial directions

$P_{r} \quad$ production rate from the residual kinetic energy $\left(\mathrm{m}^{2} / \mathrm{s}^{2}\right)$

$p \quad$ pressure $\left(\mathrm{N} / \mathrm{m}^{2}\right)$

$\bar{p} \quad$ mean pressure $\left(\mathrm{N} / \mathrm{m}^{2}\right)$

$p^{\prime} \quad$ fluctuating pressure $\left(\mathrm{N} / \mathrm{m}^{2}\right)$

$\hat{p} \quad$ filtered pressure $\left(\mathrm{N} / \mathrm{m}^{2}\right)$

$R \quad$ radius or center line of the pipe (m)

$R_{i j} \quad$ Reynolds stresses $\left(\mathrm{N} / \mathrm{m}^{2}\right)$

$R_{i j}(r, x) \quad$ two-points velocity correlations $\left(\mathrm{m}^{2} / \mathrm{s}^{2}\right)$

$R a \quad$ Rayleigh number

Re Reynolds number

$R e_{x} \quad$ Reynolds number based on the characteristic length $x$

$R e_{\tau} \quad$ Reynolds number based on the frictional velocity

$R e_{\lambda} \quad$ Taylor scale Reynolds number

$R e_{l} \quad$ Reynolds number based on the system or largest eddy length scale

$R e_{\eta} \quad$ Reynolds number based on the Kolmogorov length scale

$\bar{S} \quad$ filtered strain rate $\left(\mathrm{s}^{-1}\right)$

$\bar{S}_{i j} \quad$ filtered rate of stress tensor $\left(\mathrm{s}^{-1}\right)$

$S_{i j} \quad$ rate of strain tensor $\left(\mathrm{s}^{-1}\right)$

$\hat{S} \quad$ filtered strain rate in explicit scheme $\left(\mathrm{s}^{-1}\right)$

$\tilde{S}_{i j} \quad$ filtered strain rate tensor in explicit scheme $\left(\mathrm{s}^{-1}\right)$ 


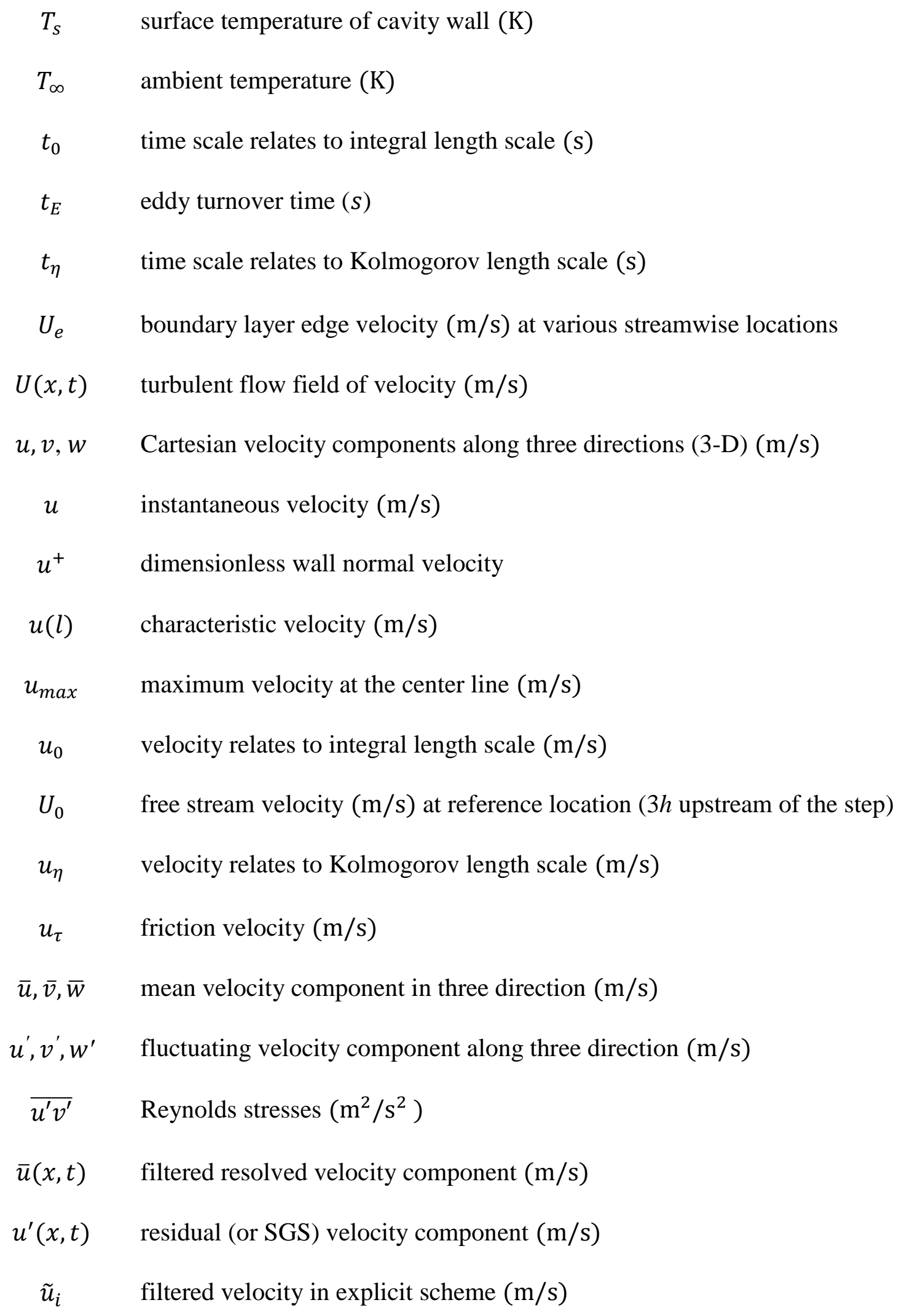


$\left\langle\tilde{u}_{i}\right\rangle \quad$ ensemble average of the filtered velocity $(\mathrm{m} / \mathrm{s})$

$\hat{u} \quad$ filtered velocity in LES $(\mathrm{m} / \mathrm{s})$

$u^{\prime} \quad$ fluctuating part of the velocity in LES $\left(u_{\text {Resolved }}^{\prime}+u_{\text {Residual }}^{\prime}\right)(\mathrm{m} / \mathrm{s})$

$W_{j} \quad$ given weights to the cell within the filter width

$x \quad$ position $(\mathrm{m})$

y Cartesian coordinate

$y^{+} \quad$ dimensionless wall normal unit

$y_{\text {crossover }}$ smallest distance which separates two layers of the boundary layer (m)

\section{Greek symbols}

$\alpha \quad$ test and grid filter width ratio $(\widehat{\Delta} / \Delta)$

$\alpha \quad$ thermal diffusivity of fluid in cavity flow $\left(\mathrm{m}^{2} / \mathrm{s}\right)$

$\alpha \quad$ closure coefficient in Cebeci-Smith model considered as 0.0168

$\alpha, \beta \quad$ arbitrary initial values in turbulent pipe flow

$\alpha_{i j} \quad$ velocity gradient in Vreman's model

$\beta \quad$ thermal expansion coefficient in cavity flow $\left(\mathrm{K}^{-1}\right)$

$\beta, c_{\eta} \quad$ constants in model spectrum based on Taylor scale Reynolds number $\left(R e_{\lambda}\right)$

$\delta \quad$ boundary layer thickness $(\mathrm{m})$

$\delta x, \delta y, \delta z \quad$ grid (or mesh) cell size in three direction (3-D) (m)

$\delta_{i j} \quad$ Kronecker delta

$\delta_{v}^{*} \quad$ velocity thickness $(\mathrm{m})$

$\Delta \quad$ spatial filter width in LES (m)

$\widehat{\Delta} \quad$ explicit filter width larger than the grid size (m) 


\begin{tabular}{|c|c|}
\hline$\varepsilon$ & dissipation of turbulent kinetic energy $(\mathrm{TKE})\left(\mathrm{m}^{2} / \mathrm{s}^{3}\right)$ \\
\hline$\varepsilon_{f}$ & filtered dissipation energy $\left(\mathrm{m}^{2} / \mathrm{s}^{3}\right)$ \\
\hline$\xi$ & relative error at an individual point \\
\hline$\xi_{m}$ & resultant mean relative error \\
\hline$\Phi_{\mathrm{ij}}(\kappa)$ & velocity spectrum $\left(\mathrm{m}^{2} / \mathrm{s}^{2}\right)$ \\
\hline $\mathcal{L}_{i j}$ & Leonard stress term known as 'Germano identitity' $\left(\mathrm{N} / \mathrm{m}^{2}\right)$ \\
\hline$\lambda$ & Taylor microscale (m) \\
\hline$\mu$ & molecular viscosity of the fluid (kg/m.s) \\
\hline$\mu_{T}$ & turbulent molecular viscosity of the fluid $(\mathrm{kg} / \mathrm{m} . \mathrm{s})$ \\
\hline$\eta$ & Kolmogorov length scale (m) \\
\hline$v$ & kinematic viscosity of fluid $\left(\mathrm{m}^{2} / \mathrm{s}\right)$ \\
\hline$v_{T}$ & turbulent kinematic viscosity of fluid $\left(\mathrm{m}^{2} / \mathrm{s}\right)$ \\
\hline$v_{\text {mix }}$ & kinematic viscosity of mixing length $\left(\mathrm{m}^{2} / \mathrm{s}\right)$ \\
\hline$v_{t_{\text {inner }}}$ & viscosity near the wall $\left(\mathrm{m}^{2} / \mathrm{s}\right)$ \\
\hline$v_{t_{\text {outer }}}$ & viscosity outer boundary layer $\left(\mathrm{m}^{2} / \mathrm{s}\right)$ \\
\hline$\rho$ & density $\left(\mathrm{kg} / \mathrm{m}^{3}\right)$ \\
\hline$\sigma_{k}$ & turbulent Prandtl number for kinetic energy \\
\hline$\sigma_{\varepsilon}$ & turbulent Prandtl number for dissipation \\
\hline $\mathcal{R}$ & reference values \\
\hline$\psi$ & predicted values from numerical solution \\
\hline$\tau_{i j}$ & shear stress tensor $\left(\mathrm{N} / \mathrm{m}^{2}\right)$ \\
\hline$\tau_{i j}^{r}$ & residual shear stress $\left(\mathrm{N} / \mathrm{m}^{2}\right)$ \\
\hline
\end{tabular}




$\begin{array}{cl}\tau(l) & \text { characteristic timescale }(\mathrm{s}) \\ \tau_{\eta} & \text { Kolmogorov timescale }(\mathrm{s}) \\ \tau_{0} & \text { integral length scale timescale }(\mathrm{s}) \\ \tau_{w} & \text { wall shear stress }\left(\mathrm{N} / \mathrm{m}^{2}\right) \\ \tau_{i j}^{\text {sgs }} & \text { sub grid scale shear stress }\left(\mathrm{N} / \mathrm{m}^{2}\right) \\ \tau_{i j}^{\text {Resolved }} & \text { resolved stress part of SGS model }\left(\mathrm{N} / \mathrm{m}^{2}\right) \\ \tau_{i j}^{\text {Residual }} & \text { residual stress part of SGS model }\left(\mathrm{N} / \mathrm{m}^{2}\right)\end{array}$

\section{Superscripts}

〈. spatial averaging

\section{Acronyms}

BLT Boundary layer thickness

CFD Computational fluid dynamics

DNS Direct numerical simulation

FGR Filter to grid spacing ratio

LES Large eddy simulation

NS Navier-Stokes equations

$P D E \quad$ Partial differential equation

RANS Reynolds averaged Navier-Stokes simulation

SGS Sub-grid scale

SSM Standard Smagorinsky model

TDMA Tri-diagonal matrix algorithm 


\section{Chapter 1}

\section{Background Theory and Scope}

\subsection{Introduction}

It is vital that fire safety engineers are able to predict the growth and spread of building-fires under a wide range of scenarios in order to design fire protection systems. It is also important for emergency organisations to predict similar effects during bushfires under a wide range of topographies, climates and types of vegetation for their resource allocation planning. Compuational fluid dynamics (CFD)-based three-dimensional predictive fire models are highly desirable for both groups of professionals. While pyrolysis (gasification of solid fuel) and combustion (chemical reaction between gasified fuel and oxygen) sub-models are distinguishing features of a CFD-based fire model, its background sub-models of mass, momentum and energy are fundamentally important.

Numerical simulation becomes more complex when a flow field becomes turbulent. Almost every flow scenario of practical importance is turbulent in nature. Turbulent flow is characterized by random fluid motions from small scale to large scale motions at various frequencies. Although the largest scale in fire scenarios can be ranged from tens of meters (building fires) to many kilometers (bushfires), the phenomena that determine their behaviour and accuracy are dependent on the scales that may be tiny fractions of a millimeter. To capture these tiny motions the spatial and temporal resolutions in numerical simulation need to be extremely fine, which requires enormous computational resources. A simulation scheme to solve mass, momentum and energy equations with such fine resolution is known as direct numerical simulation (DNS). To 
reduce the computational requirements, various numerical schemes have emerged. They fall into two broad categories, Reynolds averaged Navier-Stokes (RANS) simulation, and large eddy simulation (LES). More details of the mathematical formulation of the governing equations and numerical approaches to solving these governing equations in DNS, RANS and LES are discussed in several sections of this chapter.

\subsection{Numerical approaches to simulating turbulent flows}

The main concept of numerical simulation of turbulent flow using CFD is that it approximates fluid motions by solving a set of non-linear partial differential equations (PDEs). These differential equations consist of continuity equations (mass conservation) and momentum balance equations (Navier-Stokes equations) derived from Newton's second law of motion. For compressible flow, an additional energy equation is required to extract the necessary information about the density and temperature (Pope 2000). In the momentum and energy equations, convective non-linear terms appear which make them intractable to solve analytically.

In numerical approaches, these governing partial differential equations are discretized in space and time in the computational domain of a given physical problem using different types of numerical schemes. As mentioned above, there are three broad numerical approaches for solving Navier-Stokes equations: DNS, RANS and LES.

\subsubsection{Direct Numerical Simulation (DNS)}

DNS solves the Navier-Stokes equations by resolving all the spatial and temporal scales of turbulence present in the flow, and this results in particularly accurate solutions. The continuity and the Navier-Stokes equations for incompressible flow are as follows:

$$
\frac{\partial u}{\partial x}+\frac{\partial v}{\partial y}+\frac{\partial w}{\partial z}=0
$$




$$
\begin{gathered}
\rho\left(\frac{\partial u}{\partial t}+u \frac{\partial u}{\partial x}+v \frac{\partial u}{\partial y}+w \frac{\partial u}{\partial z}\right)=F_{x}-\frac{\partial p}{\partial x}+\mu\left(\frac{\partial^{2} u}{\partial x^{2}}+\frac{\partial^{2} u}{\partial y^{2}}+\frac{\partial^{2} u}{\partial z^{2}}\right) \\
\rho\left(\frac{\partial v}{\partial t}+u \frac{\partial v}{\partial x}+v \frac{\partial v}{\partial y}+w \frac{\partial v}{\partial z}\right)=F_{y}-\frac{\partial p}{\partial y}+\mu\left(\frac{\partial^{2} v}{\partial x^{2}}+\frac{\partial^{2} v}{\partial y^{2}}+\frac{\partial^{2} v}{\partial z^{2}}\right) \\
\rho\left(\frac{\partial w}{\partial t}+u \frac{\partial w}{\partial x}+v \frac{\partial w}{\partial y}+w \frac{\partial w}{\partial z}\right)=F_{z}-\frac{\partial p}{\partial z}+\mu\left(\frac{\partial^{2} w}{\partial x^{2}}+\frac{\partial^{2} w}{\partial y^{2}}+\frac{\partial^{2} w}{\partial z^{2}}\right)
\end{gathered}
$$

Here, $u, v$ and $w$ are the velocity components along three directions; $F$ is the external force, $p$ is the pressure and $\mu$ represents the molecular viscosity of the fluid. In tensor form, these governing equations can be simply expressed as

$$
\begin{gathered}
\frac{\partial u_{i}}{\partial x_{i}}=0 \\
\rho\left(\frac{\partial u_{i}}{\partial t}+u_{j} \frac{\partial u_{j}}{\partial x_{j}}\right)=F_{i}-\frac{\partial p}{\partial x_{i}}+\mu\left(\frac{\partial^{2} u_{i}}{\partial x_{j} \partial x_{j}}\right)
\end{gathered}
$$

In DNS, neither turbulence modelling of effects of small scales on large scales nor the averaging approach to obtain mean and fluctuating components is involved. Since it is the most accurate numerical approach to capture the turbulence, in many cases it is considered as an alternative to physical experimentation. For instance, where experimental results are not available, the DNS results are considered as the most accurate numerically obtained solutions for validating the results from numerical simulations with turbulence modelling.

The major issue that affects the practical use of DNS results is the higher computational requirements. In DNS the grid spacing must be on the same lengthscale as the smallest eddies. Hence, details of turbulent flows that occur in many practical situations can be captured only by using a very large number of grid points. This is computationally resource intensive. In addition, the required grid resolutions depend on the Reynolds number $(R e)$ which is a dimensionless quantity defined as the ratio of inertial forces to viscous forces based on the system lengthscale. 
When the $R e$ increases, the ratio between the integral lengthscale and smallest lengthscale also increases (Pope 2000) (for more details about scales please refer to Section A.3 of Appendix A). To date the largest DNS performed of an idealised channel flow are of order $R e_{\tau} \sim 5200$ (Lee and Moser 2015).

In DNS, all of the smallest scales that are responsible for energy dissipation are captured. In those cases, the computational grid size should be small enough to capture these viscous effects of flow. The grid size should be smaller than the size of the smallest eddies and this ranges from less than a millimetre (George 2005) in all practically important flows; the smallest length scale present in turbulent flows is known as the Kolmogorov scale, $\eta$. If we consider $L$ as the characteristic length of the computational domain, so for DNS, the number of grid points in one direction will be in the order of,

$$
N \sim \frac{L}{\eta}
$$

The Kolmogorov lengthscale is represented in terms of the kinematic viscosity $v\left(\mathrm{~m}^{2} / \mathrm{s}\right)$ and dissipation (the rate at which turbulent kinetic energy is converted into internal energy) per unit mass of the fluid $\varepsilon\left(m^{2} / s^{3}\right)$. Therefore $\eta$ is represented as; $\eta=\left(v^{3} / \varepsilon\right)^{1 / 4}$. The dissipation per unit mass can be approximated as $\varepsilon \sim u^{3} / L$, where $u$ is the characteristic velocity of the flow. Using these relations, the number of grid points in a domain required for DNS can be estimated as,

$$
N \sim\left(\frac{L}{\eta}\right)^{3} \sim\left(\frac{u L}{v}\right)^{9 / 4}=R e^{9 / 4}
$$

For this reason, DNS is not computationally feasible for high Reynolds numbers and is restricted to relatively low Reynolds numbers and is mostly used for comparatively simple fluid flow cases such as isotropic turbulence, channel and pipe flows etc. $R e$ that are encountered in most 
practical cases and complex engineering applications, DNS will not be able to provide numerical solutions by using high performance computational power that is presently available. For example, in atmospheric applications the $R e$ is of order $R e_{\tau} \sim 2.5 \times 10^{6}$ and the geometries can be quite complex such as flow over buildings (Bou-Zied et. al 2009).

\subsubsection{Reynolds Averaged Navier-Stokes Simulation (RANS)}

The Reynolds averaged Navies-Stokes simulation (RANS) is one of the most widely used numerical approaches to simulate turbulent flows. It takes the time averaged solution of NavierStokes equations to solve the fluid flow. This idea was first introduced by Reynolds (1895). The underlying concept behind RANS is the Reynolds decomposition which separates instantaneous fluid motions into two components, namely time averaged mean values and fluctuating quantities. The auto-correlation of fluctuating components are termed as 'Reynolds stresses' which are modelled using empirically determined relationships. The time averaged NavierStokes equations in all three spatial directions can be written as,

$$
\begin{gathered}
\rho\left(\frac{\partial \bar{u}}{\partial t}+\bar{u} \frac{\partial \bar{u}}{\partial x}+\bar{v} \frac{\partial \bar{u}}{\partial y}+\bar{w} \frac{\partial \bar{u}}{\partial z}\right)=F_{x}-\frac{\partial \bar{p}}{\partial x}+\mu\left(\frac{\partial^{2} \bar{u}}{\partial x^{2}}+\frac{\partial^{2} \bar{u}}{\partial y^{2}}+\frac{\partial^{2} \bar{u}}{\partial z^{2}}\right)-\rho\left(\frac{\partial \overline{u^{\prime} u^{\prime}}}{\partial x}+\frac{\partial \overline{u^{\prime} v^{\prime}}}{\partial y}+\frac{\partial \overline{u^{\prime} w^{\prime}}}{\partial z}\right) \\
\rho\left(\frac{\partial \bar{v}}{\partial t}+\bar{u} \frac{\partial \bar{v}}{\partial x}+\bar{v} \frac{\partial \bar{v}}{\partial y}+\bar{w} \frac{\partial \bar{v}}{\partial z}\right)=F_{y}-\frac{\partial \bar{p}}{\partial y}+\mu\left(\frac{\partial^{2} \bar{v}}{\partial x^{2}}+\frac{\partial^{2} \bar{v}}{\partial y^{2}}+\frac{\partial^{2} \bar{v}}{\partial z^{2}}\right)-\rho\left(\frac{\partial \overline{u^{\prime} v^{\prime}}}{\partial x}+\frac{\partial \overline{v^{\prime} v^{\prime}}}{\partial y}+\frac{\partial \overline{v^{\prime} w^{\prime}}}{\partial z}\right) \\
\rho\left(\frac{\partial \bar{w}}{\partial t}+\bar{u} \frac{\partial \bar{w}}{\partial x}+\bar{v} \frac{\partial \bar{w}}{\partial y}+\bar{w} \frac{\partial \bar{w}}{\partial z}\right)=F_{z}-\frac{\partial \bar{p}}{\partial z}+\mu\left(\frac{\partial^{2} \bar{w}}{\partial x^{2}}+\frac{\partial^{2} \bar{w}}{\partial y^{2}}+\frac{\partial^{2} \bar{w}}{\partial z^{2}}\right)-\rho\left(\frac{\partial \overline{u^{\prime} w^{\prime}}}{\partial x}+\frac{\partial \overline{v^{\prime} w^{\prime}}}{\partial y}+\frac{\partial \overline{w^{\prime} w^{\prime}}}{\partial z}\right)
\end{gathered}
$$

In tensor form, thee equations can be expressed as

$$
\frac{\partial \bar{u}_{i}}{\partial t}+\bar{u}_{j} \frac{\partial \bar{u}_{j}}{\partial x_{j}}=F_{i}-\frac{1}{\rho} \frac{\partial \bar{p}}{\partial x_{i}}+v \frac{\partial^{2} \bar{u}_{i}}{\partial x_{j} \partial x_{j}}-\underbrace{\frac{\partial \overline{u_{\imath}^{\prime} u_{j}^{\prime}}}{\partial x_{j}}}_{\begin{array}{c}
\text { Reynolds } \\
\text { Stesses }
\end{array}}
$$

where $v$ represents the kinematic viscosity; $v=\mu / \rho$.

The process of averaging inevitably results in loses of information. As a result, RANS models lose detailed information of the flow, more specifically the details of the flow smeared as it involves averaging to derive the governing equations. 


\subsubsection{Large Eddy Simulations (LES)}

In LES, turbulence contained in large lengthscales is resolved and small scale turbulence is modelled. Comparing computational costs, LES lies between DNS and RANS. The levels of turbulence modelling employed in three numerical approaches in terms of computational cost are shown in Figure 1.1. DNS captures all turbulence down to small scales, and provides fully resolved solutions which increases the computational time and cost and has been shown in Figure 1.1 (a). On the other hand, RANS takes the shortest time for the simulation compared to LES and DNS, but is unable to give detailed information on turbulence. However, LES lies between DNS and RANS in terms of computational cost and time illustrated in Figure 1.1 (b). LES is capable of simulating complex flows and geometries such as flow over bluff bodies where flow separation and rotation both take place (You et. al. 2007).

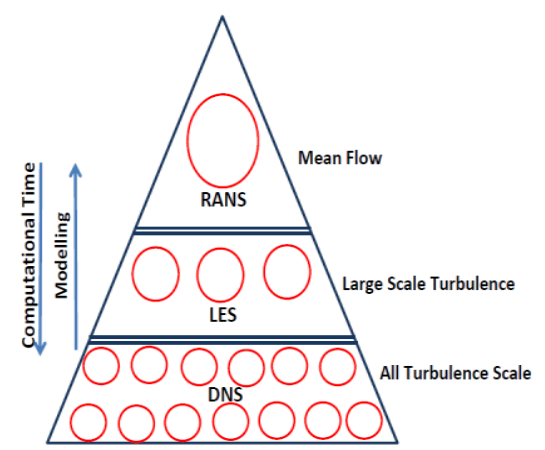

(a)

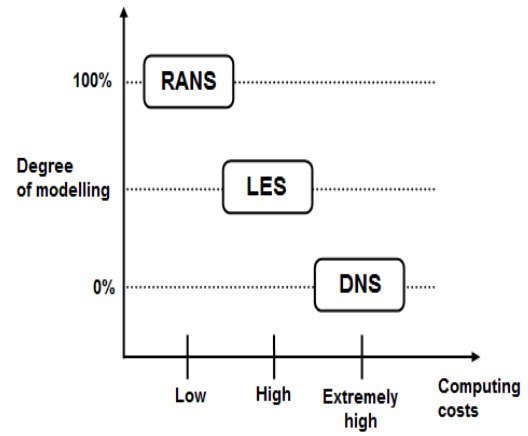

(b)

Figure 1.1. Comparison of DNS, RANS and LES in terms of (a) computational costs and (b) degree of modelling scales

As capturing small scales is computationally very expensive, in LES, large scale eddies are resolved over a number of computational cells and small scale eddies are filtered by filter width which is generally of the same as the grid size. Figure 1.2 represents how LES separates large and small eddies in physical space by using a filtering technique through the filter width $(\Delta)$. It appears from the illustrated figure that the large eddies larger than filter width (width is the same 
as grid size here) are resolved and the dissipative motions of small eddies are filtered. Effects of dissipative motion of eddies (which are smaller than the filter size) on energy containing large eddies are modelled by sub-grid scaling which saves computation time and cost compared to DNS. The resolved length scales are directly captured by the numerical scheme. On the other hand, various turbulence models (e.g. Smagorinsky model, dynamic models etc.) account for the effect that the unresolved scales has on the resolved scales. These models are also known as subgrid scale (SGS) models (Gullbrand 2004).

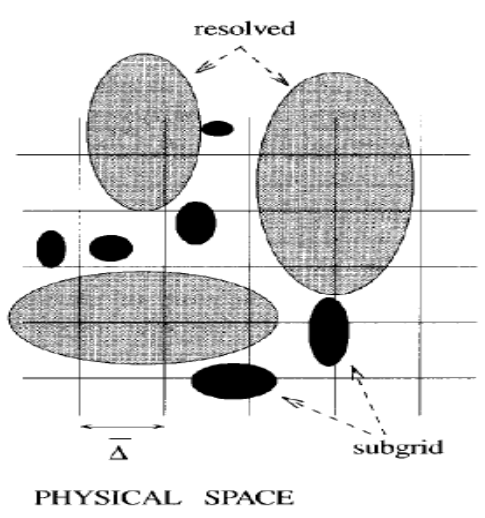

Figure 1.2. Representation of resolved and sub-grid scales (SGS)in LES (Sagaut 2001) The filtering process plays an important role in LES. Generally, LES can be characterised by two types of filtering: implicit (where filter width is same as the grid size) and explicit (where filter width is explicitly set by the modeller). However, implicit LES has a significant implication; it directly depends on the grid size. If the grid changes the model changes with grid which results in different solutions. Moreover, it has less control over the discretization errors (Mahesh et. al. 2006, Youet. al. 2007). Thus, grid convergence becomes elusive using implicit LES. Unless grid converged solutions are obtained the results of CFD simulations are of uncertain value.

\section{Subgrid-scale (SGS) models in LES}

Over the last two decades SGS models in LES have advanced significantly for complex geometries and wide variety of flows (boundary layers, wall jets, wakes, etc.). LES without SGS 
models (referred to as coarse DNS) generate unphysical results due to the accumulation of energy at the high wavenumbers. Therefore, an appropriate SGS model ensures the fidelity of LES.

The concept of SGS models was first introduced in meteorological simulations. Smagorinsky (1963) proposed an eddy viscosity model to simulate atmospheric boundary layer turbulence which was based on the Boussinesq hypothesis or approximation. This model captured the principal effects of the SGS stresses in flows. It relates turbulent shear stress to the mean flow strain rate. In some cases it is termed as gradient transport (Tennekes and Lumley 1972, Libby 1996).

$$
\overline{u^{\prime} v^{\prime}} \sim \frac{1}{2}\left(\frac{\partial \bar{u}}{\partial y}+\frac{\partial \bar{v}}{\partial x}\right)
$$

This approximation is suggested in analogous to Newton's law of viscosity with the statement that 'shear stress is proportional to strain rate', where viscosity is the proportionality constant.

$$
\tau=\mu\left(\frac{\partial u}{\partial x}\right)
$$

where $\tau$ is the local shear stress. The important fact is that this statement holds for laminar flow and is well supported by experimental results. Turbulent flow generates additional mixing of momentum and turbulent shear stress can be expressed as

$$
-\overline{u^{\prime} v^{\prime}}=v_{T}\left(\frac{\partial \bar{u}}{\partial y}+\frac{\partial \bar{v}}{\partial x}\right)
$$

where, $v_{T}$ is the turbulent viscosity. From this expression it appears that the Boussinesq hypothesis relates the comparatively small scale statistical behaviour known as Reynolds stress to large scale behaviour (statistical information extracted from mean flow behaviour). Smagorinsky used an analogous idea to develop an eddy viscosity subgid-scale model based on this Boussinesq approximation which relates subgrid-scale stresses to the eddy viscosity. It is discussed in detail in chapter 2 section 2.2.1. The model coefficient (known as the Smagorinsky 
constant) was derived by Lilly (1966) for homogeneous and isotropic turbulence. Performance of the Smagorinsky model was first explored by Deardorff (1970) for three-dimensional channel flow driven by a uniform pressure gradient at high Reynolds number $\left(R e_{H} \approx 240,000\right.$ based upon the channel height). From the study, he concluded that the agreement between the computed statistics and the experimental data of Laufer (1954) was in poor agreement and the accuracy of the numerical approach was expected to be improved with an increase of numerical resolution.

One of the challenges associated with LES is to model the effects of small scale eddies and to derive governing equations inclusive of them. To address the modelling problem, Leonard (1974) proposed the derivation of LES governing equations using the filtering concept. The concept of filter kernel is to separate small and large scale eddies by applying a spatial filter to the velocity field. A volume averaged filter was formulated by Schumann (1975) and applied to the simulation of incompressible turbulent channel flows. In his proposed model, SGS stresses are divided into two parts, one is accounting for isotropic turbulence and the other for anisotropic effects of turbulence. Schumann concluded that the results with the SGS model were in good agreement with the channel flow experimental data. The concept of spectral eddy viscosity was presented by Kraichnan (1976) and applied to isotropic turbulence. Using this model, he showed the limitations of the eddy viscosity concept to represent the effects of small scale turbulence. The extension of Kraichnan's work was carried out by Leslie and Quarini (1979) where they presented a theoretical formulation of SGS modelling procedures. From this study, they supported Kraichnan's findings that the effective eddy viscosity varies with the wavenumbers associated with different eddy lengthscales. They tested the SGS model for isotropic flow and found the results were consistent with that of Kraichnan (1976). Clark et al. (1979) studied SGS 
tensor formulations for periodic homogeneous isotropic turbulence at low Reynolds number. They restricted their study to Reynolds number (based on the Taylor microscale) in the range of $R e_{\lambda} \leq 40$ due to lack of computational capacity. Simulation results showed that large scale eddies were well captured by energy spectrum analysis but the performance of the SGS model was only moderately good. The idea of scale-similarity was first introduced by Bardina et al. (1980). This idea helps to express the sub-grid field quantities in terms of filtered quantities. The study indicates that information in the resolved scales is sufficient to describe some characteristics of the fluid flow and the argument 'production equals dissipation' does not applicable to small scale turbulence decay. Another feature of this model is that it produces backscatter (energy transfer from small scale to large scale eddies) of energy. However, from simulations it is found that the model does not dissipate enough energy which leads to inaccurate results. This problem was addressed by Germano et al. (1991), and they proposed a dynamic SGS modelling approach where a model coefficient is not prescribed but it is computed dynamically. LES with the proposed dynamic SGS model was used to simulate a fully developed turbulent channel flow. Germano et al. (1991) found the results were in good agreement with the DNS results of Kim et al. (1987). However, the mathematical formulation of dynamic SGS model has some inconsistencies and it is restricted to flows that are statistically homogenous in at least one direction, a fact identified by Goshal and Moin (1995). They rectified the inconsistency of the mathematical formulation of Germano's work and studied filter inhomogeneity for isotropic turbulence and found the obtained results are in good agreement with experiments. Goshal (1996) addressed the issues of discretization error (generated from finite differencing) and aliasing errors (generated from non-linear terms in SGS) in LES using a dynamic SGS model to simulate isotropic turbulence. As remedies for the problem of clipping 
(cutoff or discard negative model coefficients) negative values and to minimise the singularities in dynamic modelling, Meneveau et al. (1996) proposed a dynamic SGS model in which SGS stresses are formulated in Lagrangian way by using first-order Euler time integration and linear interpolation in space. The constant and dynamic Smagorinsky models and their variations are most widely used SGS models. The interested reader can refer to the books of Sagaut (2001), Pope (2000) and Wilcox (1993) for an extensive review of SGS models used in LES.

\section{Numerical methods in LES}

Numerical methods play an important role in ensuring the accuracy of simulations. Numerical schemes that are used for LES often rely on finite-difference methods or spectral methods as they are computationally less expensive than finite element and finite volume methods (Goshal 1996). LES is prone to numerical errors that arise from discretization and aliasing, that are directly dependent on the numerical schemes.

Goshal (1996) studied numerical errors in LES for various finite difference methods (from $2^{\text {nd }}$ order to $8^{\text {th }}$ order) and spectral methods. He found that higher order schemes lead to reduction in the numerical errors. Kravchenko and Moin (1997) studied the effects of numerical errors in LES by performing numerical simulation of turbulent channel flow using finite difference methods and spectral methods. Numerical and analytical studies show that aliasing errors are more destructive for spectral and fourth and sixth-order difference than for second-order finitedifference simulations. They assumed the probable reason is aliasing errors (generated from the non-linear terms with finite differencing) may cause numerical instability and excessive turbulence decay for both spectral and higher-order numerical schemes compared to lower-order numerical scheme for the test case. Further investigations of Goshal (1996) were carried out by Moin and Chow (2003) where they gave emphasis to numerical as well as modelling errors that arise from LES. The authors studied decaying isotropic turbulence using a finite difference 
method. Findings of their study are consistent with those of Goshal (1996) that higher order numerical schemes have more control over numerical errors. Gullbrand and Chow (2003) presented the effects of numerical errors of second and fourth order finite difference schemes to assess the performance of implicitly and explicitly filtered LES (discussed in Section 1.3.1 and 1.3.2, respectively) for various SGS models. They observed the difference between the simulation results and reference data for different SGS models was larger for the fourth order than for the second order code. They assumed this may be due to the coarser resolution used in the fourth-order code compared to the resolution of the second-order code. Kempf et al. (2011) studied numerical error of the turbulent non-premixed bluff-body flame using the Smagorinsky model in LES, where the error is defined with respect to experimental data. From their study they suggested that second-order scheme can be used in anisotropic turbulence in complex geometries to obtain reasonable LES solutions. Keskinen et al. (2016) carried out numerical investigations using four different SGS approaches for a three-dimensional, turbulent pipe flow $\left(R e_{\tau}=360\right)$. SGS models are the standard Smagorinsky, linear interpolation (non-dissipative), the Gamma limiter (dissipative), and the scale-selective discretization (slightly dissipative). In their study, they have used a second-order numerical scheme for the SGS models they considered. Out of four SGS models, the authors found that that Smagorinsky model shows the best agreement with the reference data.

\subsection{Contribution of filtering schemes in LES}

According to the classical theory of Kolmogorov, lengthscales of eddies in turbulent flows can be divided into three regions (Pope 2000). It is considered that the largest eddies are the same size as the characteristic lengthscales of the flow geometry, and should generally depend on 


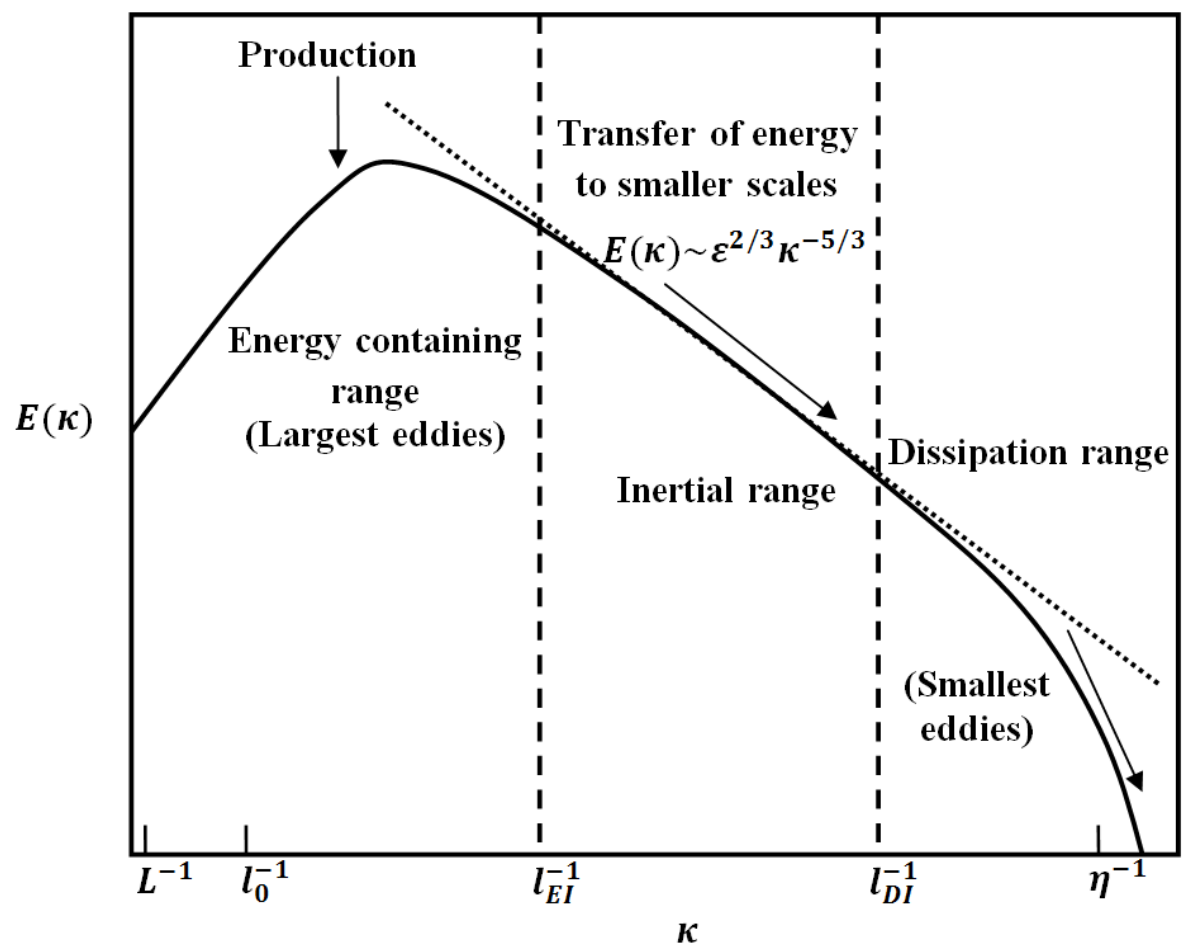

Figure 1.3. Energy cascading process from largest eddy scales to smallest eddy scales in terms of turbulent kinetic energy (vertical) and wavenumbers (inversely proportional to the length scales) plotted in log-log scales

the fluid properties. This part in the energy spectrum of turbulence is known as the energy containing range. Kolmogorov lengthscales are the smallest eddy scales. In these scales the influence of viscosity is dominant and energy is transferred to surroundings by dissipation in the form of heat: the local Reynolds number (Reynolds numbers calculated on the basis of eddy length scales) is of the order of unity. Between these largest and smallest scales, other scales of eddy exist and these eddy length scales can be expressed by comparatively large local Reynolds numbers: they are independent of viscous effects. This intermediate class is known as inertial subrange scales; they depend on the dissipation rate only and are fully independent of the types of flow (whether wall bounded or free shear flow).

The intermediate and smallest eddy length scales are universal and they show an isotropic nature in all types of flow. Illustrations of these three types of lengthscales are presented in Figure 1.3 
in terms of wavenumbers (related to wavelength or eddy length scales and inversely proportional to the characteristic lengthscale of an eddy) and the specific kinetic energy spectrum plotted on logarithmic axes. This process of transferring energy from large eddies to smaller eddies is known as an energy cascading process. It illustrates how energy is transferred between eddies over a wide range of lengthscales. It was first introduced by Richardson (1922). Energy is produced in the largest scale $\left(l_{0}\right)$ eddies and is transferred via the inertial range to the Kolmogorov scale $(\eta)$ where it is dissipated in the form of heat. The dissipation range is highly influenced by viscous effects and occurs in the smallest scales. Whereas $l_{E I}$ and $l_{D I}$ presents the demarcation between the energy containing and dissipation range from the inertial subrange during the energy cascading process. According to Kolmogorov, the energy transferred from the large energy containing range to the inertial range is equal to the energy transferred from the inertial range to the dissipation range or Kolmogorov scales and further into heat (Pope 2000). Advantage of this energy cascading property is taken in LES of turbulent flows where the effects of the smallest scales on the largest scales are modelled as turbulent dissipation (Sagaut 2001). Extended studies of LES and their most important aspects to resolve turbulence in many practical and complex situations are discussed in the later sections of this chapter.

\subsubsection{Implicit LES schemes}

A large number of authors showed that implicit schemes are highly grid sensitive and fail to control numerical errors (more specifically discretization errors). Higher order numerical schemes in implicit LES were studied by Lund and Kaltenbench (1995) for turbulent flow inside a pipe. They observed grid sensitivity even with a high order numerical scheme applied to implicitly filtered LES. Sensitivity of implicitly filtered LES to the numerical grid due to the inherent dependence of the filtering operation on the discretized operators is assessed by 
Kravchenko and Moin (2000) in their numerical studies of flow over a circular cylinder at a low Reynolds number $\left(R e_{D}\right) 3900$. They used both the dynamic and the standard Smagorinsky model to conduct the implicitly filtered LES simulations. After comparing their results with experimental data, they concluded that grid converged solutions are difficult to obtain by using implicit LES even at very low Reynolds number fluid flow. Meyers \& Sagaut (2007) conducted a numerical study of channel flow at low Reynolds numbers $\left(R e_{\tau}=298\right)$ using implicitly filtered LES, and compared their results with DNS. After evaluating the performance of a number of SGS models, they concluded that grid sensitivity remained, even when the grid was very finely resolved.

\subsubsection{Explicit LES schemes}

Implicitly filtered LES can certainly converge to the limit of a DNS resolution, but it will in general not converge to that limit monotonically thus complicating convergence analysis as the literature reference above demonstrates. In the last two decades, explicit filtering schemes have been investigated by various researchers in which the filter width is maintained constant thus keeping the model unchanged while the numerical error is reduced by refining the grid (Carati et al. 2001; Winckelmans et al. 2001). Explicitly filtered LES converges monotonically allowing for a systematic approach to testing the grid sensitivity of the solutions (Matthew et al. 2003). Ghosal and Moin (1995) and Ghosal (1996) discuss the advantages of explicit filtering over implicit filtering in LES for isotropic turbulence inside a cubic configuration with periodic boundary conditions. Najjar and Tafti (1996) simulated turbulent channel flow using an explicit scheme. In their study, the focus was on the control of numerical errors that are generated due to using explicit LES. They used different test filters (top hat and Fourier cut-off filters) in a dynamic eddy viscosity model to account for the turbulence. The test filters were based on grid 
spacing obtained from DNS resolution of a channel flow study conducted by Kim et al. (1987).

Simulated results were compared with DNS where explicit LES was observed to provide more accurate solutions than implicit LES.

Gullbrand (2002) studied grid independent LES for turbulent channel flow using explicit filtering. She concluded that an explicit filter is able to provide grid independent results for turbulent channel flow and to significantly reduce the numerical errors which are associated with high wavenumbers generated from the convective term of the Navier-Stokes equations. The case study was further expanded by Gullbrand and Chow (2003) to assess the errors associated with different numerical schemes as well as SGS turbulence closures. They provided guidelines on filter width to grid spacing ratio (FGR) for a number of numerical schemes. It was found that for fourth-order finite difference schemes, an explicit filter width should be at least twice that of the grid cell, and for second-order schemes it should be at least four times. These findings are

consistent with those of Chow and Moin (2003). More recently, Radhakrishnan and Bellan (2012, 2013, 2015) have extensively studied grid independence of explicitly filtered LES for single- and two-phase compressible fluid flows with evaporation inside an internal combustion chamber. Prior to conducting grid independence tests, a DNS solution database was created which the authors termed a 'trusted template' for validating the LES results. A DNS study was carried out because it was not possible to conduct experiments of the proposed test cases due to complex boundary conditions.

\subsubsection{Selection of filter width in explicit LES scheme}

The selection of the filter width in explicit LES schemes has often been either inadequately addressed, or in some cases completely ignored by previous researchers. Some authors do, however, mention the selection of the filter width. Gullbrand (2002) proposed a 'true' LES 
approach which means that a grid-converged LES solution is obtained by refining the grid while the filter width (taken from a DNS study) is unchanged. From her study, it appears that she took the filter width as being eight times the DNS grid resolution $\left(\Delta=8 \partial x_{D N S}\right)$ although the rationale for this is unclear. There were two computational grid resolutions selected for the simulation: one was taken as $\partial x_{\text {coarse }}=4 \partial x_{D N S}$ corresponding to an FGR of two, and the second was $\partial x_{\text {fine }}=2 \partial x_{D N S}$ corresponding to an FGR of four. A similar case study was performed by Gullbrand and Chow (2003) to assess the effect of numerical errors arising from different numerical schemes. They ran implicit LES simulations with four coarse grid resolutions: $6 \partial x_{D N S}, 4 \partial x_{D N S}, 3 \partial x_{D N S}$ and $2 \partial x_{D N S}$ finding that $3 \partial x_{D N S}$ was the coarsest resolution that gave the same results as those given by DNS with the grid size resolved to the Kolmogorov length scale, and it was selected as the grid size required for explicit LES. However, based on the assumption that a fourth-order numerical scheme would be more accurate than the second-order scheme, a coarser grid, i.e. $4 \partial x_{D N S}$ was selected for the simulation in which a fourth order scheme was implemented, whereas for the second order scheme it was maintained at $3 \partial x_{D N S}$. In the cases of both numerical schemes they kept the filter width fixed at $\left(8 \partial x_{D N S}\right)$, leading to an FGR of two, and nearly three for the fourth- and second-order schemes, respectively. Radhakrishnan and Bellan $(2012,2013,2015)$ followed a very similar approach in selecting the filter width in explicit LES schemes. They selected three different meshes (coarse, $\partial x_{\text {coarse }}=$ $4 \partial x_{D N S}$ with an FGR of two, medium, $\partial x_{\text {medium }}=2 \partial x_{D N S}$, with an FGR of four and fine, $\partial x_{f i n e}=\partial x_{D N S}$, with an FGR of eight). For higher order numerical schemes the filter width was taken as 8 times that of the DNS grid resolution. The literature indicates that a similar approach has been adapted in almost every explicit scheme test case where filter widths are selected from existing or created DNS solutions. 


\subsubsection{Filter to grid spacing ratio (FGR) in explicit schemes}

Filter to grid spacing ratio (FGR) plays an important role in providing appropriate solutions in explicit LES schemes. This issue has been reported by several authors by performing various fluid flows using explicit schemes. Ghosal (1996) reported that significant numerical errors were generated when performing explicit LES for isotropic turbulence decay inside a cubic box. The author focused on the errors that were generated due to spatial discretization arising from finite differencing schemes of different orders. One of the notable findings of his study was that a significant portion of the numerical errors arose from the non-linear and sub-grid terms in LES. According to their approximation the aliasing errors (errors from non-linear terms) are independent of finite difference schemes. An extension of the work of Goshal (1996) was carried out by Chow and Moin (2003). They investigated the numerical errors for a similar case study using both low order (second order) and higher order (fourth and sixth order) explicit LES codes. Their suggestions were that for a second-order finite difference scheme, the desired filter-grid ratio is at least four and for a higher order (i.e. fourth or sixth order) numerical scheme an filtergrid ratio of two is sufficient. Turbulent channel flow using explicit filtering was investigated by Gullbrand and Chow (2003) to assess the effect of numerical errors for second- and fourth-order numerical schemes. Furthermore, the case study was also investigated for various turbulence models and grid resolutions. In this work, for fourth-order finite difference schemes the explicit filter width an filter-grid ratio of two was found to be sufficient; for the second order it was four, which supported the findings of Chow and Moin (2003).

\subsection{Research gap and limitations}

From the above survey of the literature, it is found that almost all the case studies with explicit LES have been conducted for very simple fluid flow cases (such as channel flow, pipe flow, flow 
over a circular cylinder, etc.) and have not been used in any practical cases or in engineering applications (Lee and Moser 2015). For simple flow cases, well-developed DNS reference data are either available or can be generated without much difficulty. Therefore, it was straightforward to choose the grids and/or filter width (explicit scheme) for LES, based on the available/required DNS grid resolutions. Furthermore, there are no guidelines available to select the filter width for other complex fluid flow cases where no reference resolutions (DNS) are available. DNS cannot be easily performed to determine the filter width for explicit LES for practical cases. An important question in the absence of DNS resolution is how to select the filter width which is a purely model parameter in explicitly filtered LES. Can it be based on a percentage of a physical parameter such as the boundary layer thickness (BLT), which is considered the same size as the largest eddy (Tennekes and Lumley 1972)? It then raises the second question: how can we select the percentage of a physical parameter and select an FGR consistent with the principles of LES and grid convergence? The main objective of this current work is to address the above issues and to develop an appropriate scheme to obtain a simulation result consistent with the principles of LES which are (a) the filter width lies within inertial range and (b) all energy containing eddies are adequately captured and grid convergence is obtained in explicit schemes (Pope 2004).

The CFD-based model fire dynamics simulator (FDS) is a promising model for simulating turbulent flows, arising from fire, with a reasonable degree of accuracy when the flow field is highly resolved (Abubakar 2015). FDS is a time-dependent, three-dimensional computational fluid dynamics CFD-based fire model used to solve the Navier-Stokes equations for turbulent flows. It is an open source LES code developed by McGrattan et al.(2011). In this study, FDS has been considered as the baseline code to simulate the test cases which is a non-iterative 
pressure solver to couple the pressure and velocity fields using a predictor and corrector method. It employs a kinetic energy preserving, second-order central differencing scheme for spatial discretization of the momentum equation. FDS uses block structured staggered Cartesian grids and data storage for primitive variables (Harlow and Welch 1965) where scalar quantities are stored at cell centres and velocity components are stored at their respective face centres (Mcdermott 2014).

It is known that higher order numerical schemes such as fourth order or sixth order numerical schemes increase the accuracy of LES by minimizing numerical errors (Ghosal 1996). However, as the higher numerical scheme is computationally very expensive, in CFD based fire models usually only a second-order scheme is used (McGrattanet al. 2011). This is fairly common throughout LES studies (Goshal 1996, Kravchenko and Moin 2000, Moin and Chow 2003, Chow and Gullbrand 2003, Radhakrishnan and Bellan 2013, Keskinen et al. 2016), the reasoning is it provides adequate solutions which converge significantly faster than higher-order numerical schemes.

However, its default mode of operation is an implicit LES scheme and results are highly dependent on grid sizes making it very difficult to carry out validation studies for practical flows (Moinuddin et al. 2010). This research is aimed at reducing grid dependency by implementing an explicit LES scheme within it so that reliable validation studies of FDS can be carried out in order to use it as a predictive tool for fire risk, so that effective mitigation strategies can be undertaken.

\subsection{Aims and research objectives}

The principal objective of this work is to develop an explicit filtering scheme to obtain an appropriate solution in LES. In this way it will become feasible to simulate wall-bounded 
turbulent flows that are practically important. This is achieved by defining the width of the filter as some function of the wall boundary layer. The aims of this research are achieved by implementing the following methodology:

- Investigate four SGS models with a second-order numerical scheme and implicit filtering to identify the most promising SGS model despite the elusiveness of grid convergence. In the process, evaluate the adequacy of the selection of the baseline code. This is achieved through simulation of a selected benchmark case (flow over a backward facing step).

- Develop an appropriate explicit filtering scheme and implement in FDS with special attention to:

a. Selection of initial filter width as a function of a physical parameter.

b. Identification of filter width to grid ratio for a second-order numerical scheme.

- Apply a systematic approach to obtain an appropriate LES solution for wall-bounded flows by integrating an SGS model with an explicit LES scheme in FDS at two Reynolds $\operatorname{numbers}\left(R e_{\lambda}\right)$ : namely, buoyancy driven flow inside a differentially heated rectangular cavity and flow over a backward facing step have been considered as low and high Reynolds number flow test cases respectively. The models have been validated against published experimental data.

FDS is appropriately modified (wherever needed) in this study to achieve these aims. Of the two cases studied, the first test case is flow over a backward facing step at a relatively high Reynolds number $\left(R e_{\lambda}=115\right)$ based on Taylor's microscale $(\lambda)$. The appropriate LES solution that is obtained is compared against the experimental work of Jovic and Driver (1992). The second case is a bouyancy driven rectangular cavity flow at a low Reynolds number $\left(R e_{\lambda}=25\right)$. The results of these simulations are compared with experimental results of Cheesewrightet al. (1986). In 
both cases, the selection of the filter width for the explicit LES scheme is taken as a function of the boundary layer thickness (BLT). With detailed analysis of energy spectra for explicit LES results, a systematic approach is proposed for obtaining a simulation result consonant with the principles of LES and grid convergence in an LES explicit scheme. 


\section{Chapter 2}

\section{Governing equations and numerical schemes}

\section{1 Introduction}

The physics of fluid flow can be characterized by equations representing the conservation of mass, momentum and energy. The equations generally assume the form of partial differential or integral equations that are solved numerically at discrete points in time and space (or computational domain). In this study, an LES scheme has been developed and used to simulate turbulent flows. In LES, the resolved scales that account for the production of energy are obtained by solving the Navier-Stokes equations directly: this allows the temporal and spatial evolution of eddies to be captured. However, the effect of unresolved small scale eddies, that account for the effect of dissipation of energy, on resolved scales are modelled. These models are known as sub-grid scale (SGS) models.

\subsection{SGS modelling of turbulence in the baseline code}

Turbulence contains a wide range of length and time scales in the flow. In LES, SGS modelling considers the effect of small scale eddies that are computationally intensive to be resolved on the computational mesh. SGS modelling represents the effect of these small scale motions on the resolved large scale fluid motions (Meneveau 1994). In LES, scales are resolved down to a predefined lengthscale $\Delta$.

The resolved and unresolved fluid flow motions are delimited by spatial filtering $(\Delta)$ in the velocity field. Spatial filtering decomposes the velocity $u(x, t)$ into resolved (or filtered) component $\hat{u}(x, t)$ and residual (or SGS) component $u^{\prime}(x, t)$ as shown in Figure 2.1. The filtered 
velocity component is time dependent and represents the motion of large eddies. When spatial filtering is applied to the Navier-Stokes equations, the momentum equation produces a new term known as a residual stress tensor (or SGS stress tensor) which accounts for the interaction of small scale fluid motions. This SGS stress tensor is accounted for by modelling the eddy viscosity.

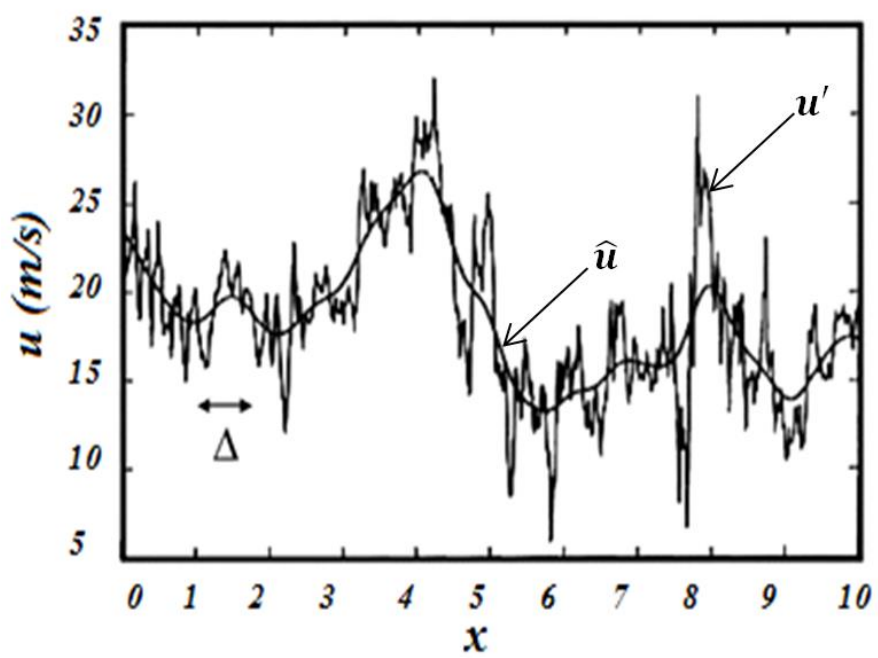

Figure 2.1. Unfiltered and filtered turbulent field showing decomposition used in large eddy simulation

The most widely used eddy viscosity model in LES was proposed by (Smagorinsky 1963). Deardorff (1972) also explored a Smagorinsky type model in meteorological applications. The main characteristic of the Smagorinsky model is its capacity to quantify the rate of energy transfer from large to small scale phenomena, and then to the smallest scales until viscous effects dominate. Moin et al. (1991) has identified several limitations of the Smagorinsky (1963) model, namely:

1. The Smagorinsky constant is not universal and it varies with the nature of the turbulent flow being modelled

2. The model does not display the correct limiting behaviour near the wall 
3. Even in the limit of laminar flow, the Smagorinsky model suggests that turbulence continues to contribute to the effective viscosity of the fluid. As a result the Smagorinsky model is too dissipative in the laminar/turbulent regime

4. The model does not account for backscatter of energy from small scales to large scales, which has been shown to be important in the transition regime

5. Compressibility effects are not included in the model

These limitations were focused on by many authors in their studies and they proposed modifications of the Smagorinsky model. Germano et al. (1991) introduced a dynamic Smagorinsky model which is capable of accounting for dissipative energy and change of model coefficients throughout the computational domain with flow variation (such as laminar to turbulent or the transition regime between laminar and turbulent flow). Vreman et al. (1994) noted that under certain flow condition the dynamic Smagorinsky coefficient goes negative. Germano et al. (1991) dealt with this condition by a clipping process that ensures the Smagorinsky coefficient remains positive. Vreman (1994) proposed an alternative eddy viscosity model that obviates this problem.

The above mentioned LES models, namely the standard Smagorinsky model, the dynamic Smagorinsky model, and the Deardorff and Vreman models are available in the default version of FDS to simulate turbulent flows. Detailed descriptions to compute eddy viscosity using these SGS models are discussed in the following sections.

\subsubsection{Standard Smagorinsky model}

The standard Smagorinsky model was developed based on the concept of the Boussinesq hypothesis (see section 1.2.3) which is extensively used in RANS models to solve turbulence. The sub-grid stress model is expressed by, 


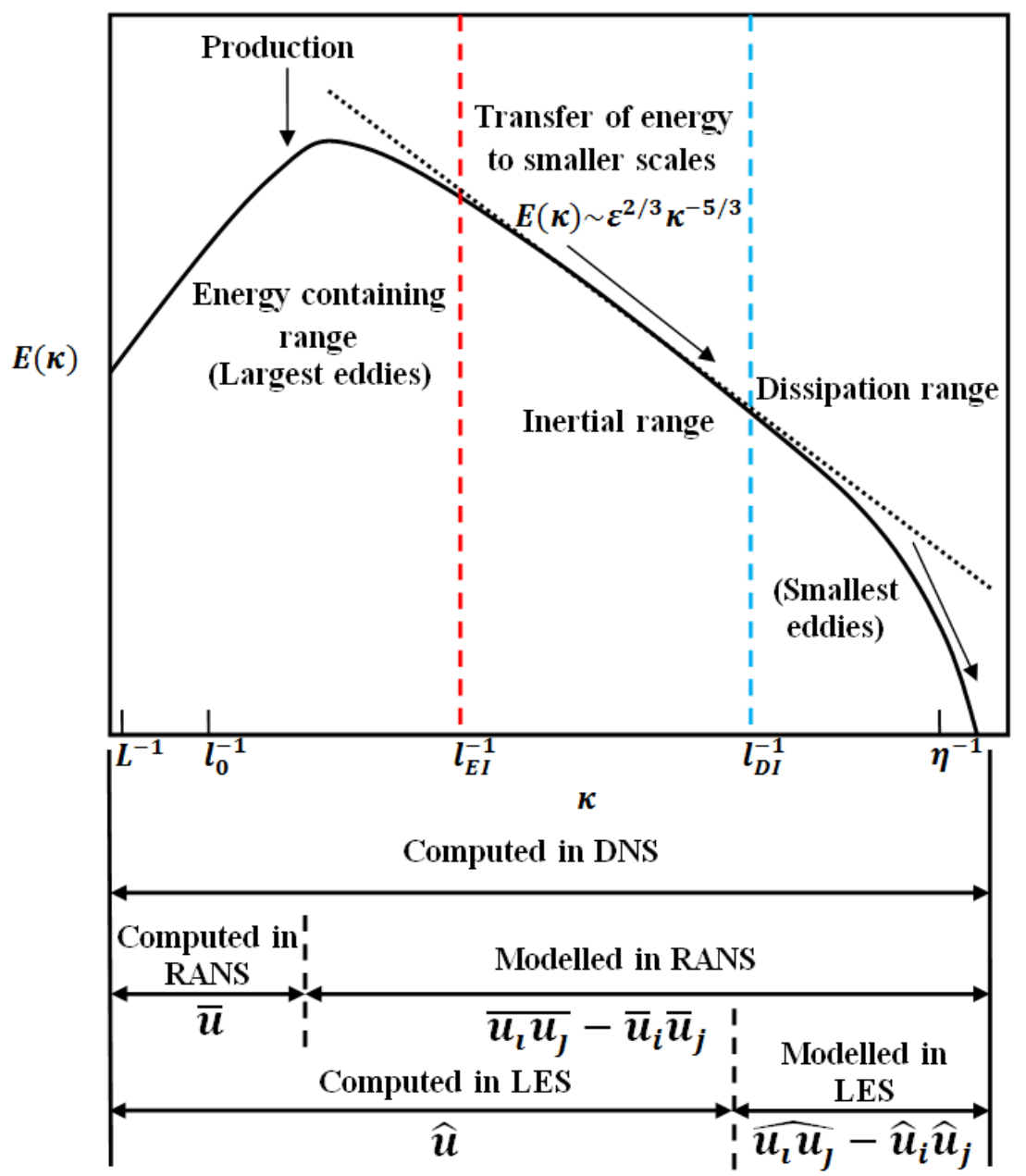

Figure 2.2. Simulation of energy cascading process in DNS, LES and RANS. Vertical dashed line $\left(\boldsymbol{l}_{\boldsymbol{E} I}^{-\mathbf{1}}\right)$ demarcates the inertial and the energy containing range and the dashed line $\left(\boldsymbol{l}_{\boldsymbol{D} I}^{-\mathbf{1}}\right)$ demarcates the inertial and the dissipation range

$$
\tau_{i j}^{s g s} \approx-2 v_{t} \hat{S}_{i j}=-2\left(C_{S} \Delta\right)^{2}|\hat{S}| \hat{S}_{i j}
$$

In this model, the SGS stress tensor $\tau_{i j}^{s g s}$ is related to filtered rate of strain (analogous to the Boussinesq approximation) where

$$
\hat{S}_{i j}=\frac{1}{2}\left(\frac{\partial \hat{u}_{i}}{\partial x_{j}}+\frac{\partial \hat{u}_{j}}{\partial x_{i}}\right)
$$

and,

$$
|\hat{S}| \equiv\left(2 \hat{S}_{i j} \hat{S}_{i j}\right)^{1 / 2}
$$

where $v_{t}$ is the eddy viscosity, $\hat{S}$ is the resolved component of the strain rate, and $\Delta$ is the filter width, which is expressed as a function of the local grid spacing. The model coefficient, $C_{S}$, 
assumes typical values in the vicinity of 0.2 . This value can be theoretically estimated for isotropic turbulence (Lilly 1966). However, the value of this model coefficient also depends on the types of filter and fluid flow. In the context of the energy cascading approach, the standard Smagorinsky model is completely dissipative in nature and assumes that the energy produced in large scales is completely transferred to small scales and that they dissipate the energy in the form of heat.

The energy cascade within the fluid follows the process shown in fig 2.2. Ideally this should be captured by physical models such as RANS, LES, or DNS, used to simulate the flow (which is an expanded version of Figure 1.3). In Figure 2.2, vertical dashed lines $l_{E I}^{-1}$ and $l_{D I}^{-1}$ in different colours, demarcate the inertial sub-range from the energy containing range and from the dissipation range.

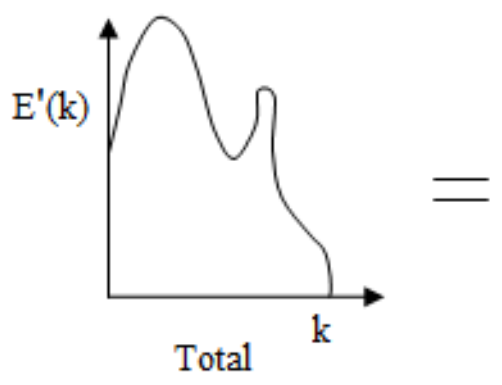

$u$

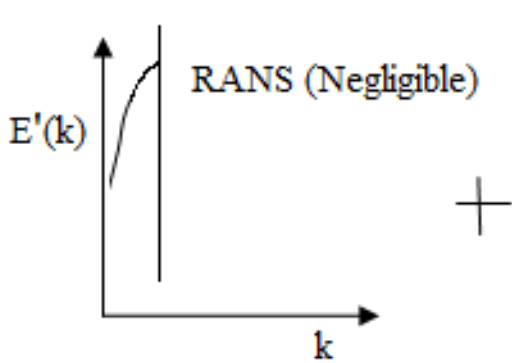

Resolved

$\bar{u}$

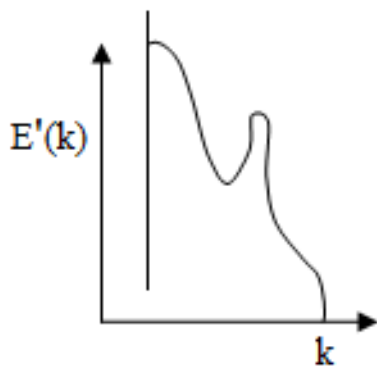

Modelled

$u^{\prime}$

Figure 2.3. Energy spectrum decomposition process in RANS

As RANS has been used for several decades since the 1970's, it may be useful describe SGS modelling (of LES) with respect to RANS. The difference between RANS and LES schemes can be demonstrated through Figures 2.3 and 2.4. The Reynolds shear stress in RANS can be constructed and expressed as,

$$
\tau_{i j}=\left\langle u_{i} u_{j}\right\rangle-\left\langle u_{i}\right\rangle\left\langle u_{j}\right\rangle=\overline{u_{\imath} u_{\jmath}}-\bar{u}_{i} \bar{u}_{j}
$$


where, $\bar{u}_{i}$ and $\bar{u}_{j}$ are ensemble averages of the velocity components which can be decomposed into $\bar{u}$ (the mean part) and $u^{\prime}$ (fluctuating part) as $u=\bar{u}+u^{\prime}$.

The instantaneous energy decomposition of RANS is shown in Figure 2.3. In the case of RANS, only the energy containing scales are solved for by the Navier-Stokes equation; the temporal average of which are manifested as mean velocities and the remaining energy is dissipated through Reynolds stresses. There is a plethora of models of these stresses.

In LES, sub-gird stress model in terms of velocity can be expressed as

$$
\tau_{i j}^{s g s}={\widehat{u_{l}}}_{J}-\hat{u}_{i} \hat{u}_{j}
$$

For LES, velocities can be decomposed into,

$$
u=\hat{u}+u_{\text {Residual }}^{\prime}=\overline{\hat{u}}+u_{\text {Resolved }}^{\prime}+u_{\text {Residual }}^{\prime}
$$

where, $u$ denote the velocity, $\hat{u}$ represents the filtered velocity, $\overline{\hat{u}}$ represents mean part of the filtered velocity and $u^{\prime}$ fluctuating part of the velocity for LES. It may be noticed that, in the case of LES, the fluctuating part of the velocity is further decomposed into components:

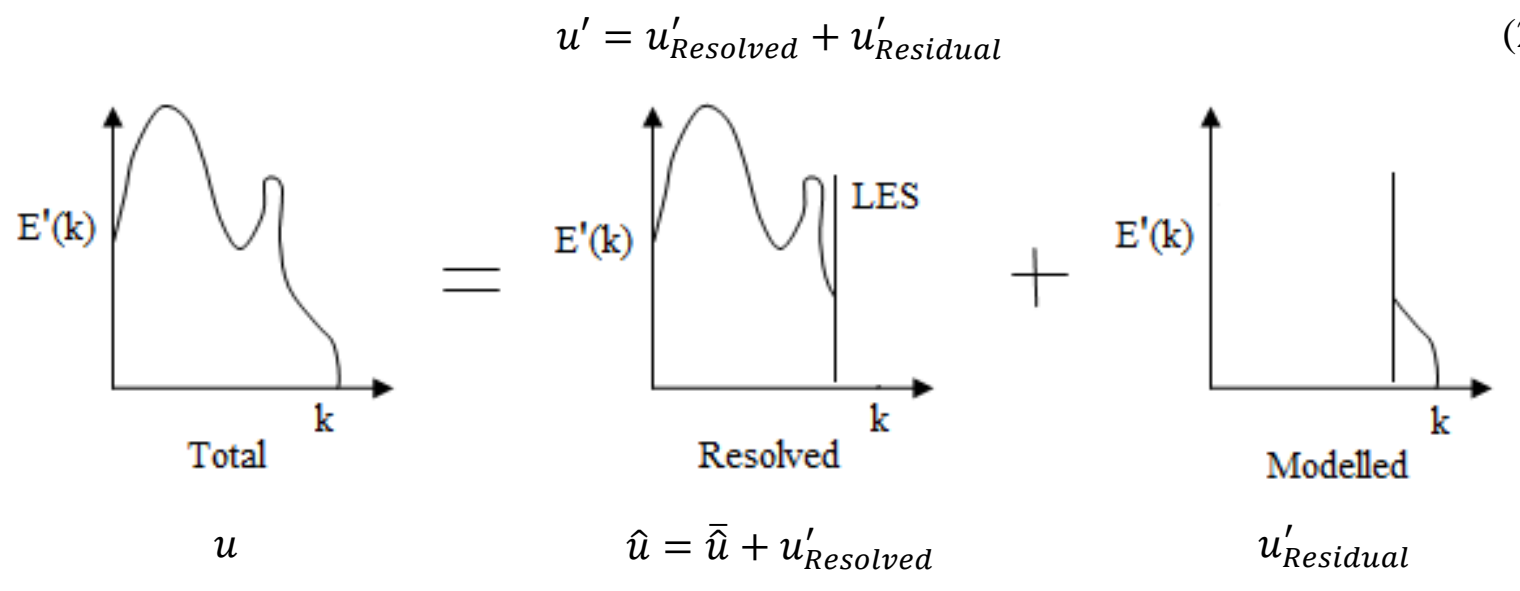

Figure 2.4. Energy spectrum decomposition process in LES

The instantaneous energy decomposition of LES is shown in Figure 2.4. The scales are directly computed up to the inertial sub-range representing the filtered velocity field. The rest is modelled by means of SGS models. The filtered velocity field obtain by convolution (Pope 2000) is as follows, 


$$
\hat{u}(x)=\int_{-\infty}^{\infty} G(r) u(x-r) d r
$$

Here, $G(r)$ is the filter function.

For homogeneous turbulence flow, $E(k)$ is the energy spectrum function when all scales are resolved (such as DNS). The energy spectrum function for LES can be denoted as $\widehat{E}(\kappa) . \widehat{G}(k)$ is the LES transfer function for converting the spatial domain into Fourier space expressed in terms of wavenumber $k$. So, the filtered energy spectrum can be written as (Pope 2000),

$$
\begin{gathered}
\widehat{E}(\kappa)=[\widehat{G}(\kappa)]^{2} E(\kappa) \\
\widehat{G}(\kappa)=\left[\frac{\widehat{E}(\kappa)}{E(\kappa)}\right]^{1 / 2}
\end{gathered}
$$

The filter functions for the box and Gaussian filters are,

$$
\begin{aligned}
\text { Box filter: } & \hat{G}(r)=\frac{1}{\Delta} H\left(\frac{1}{2} \Delta-|r|\right) \\
\text { Gaussian filter: } \hat{G}(r) & =\left(\frac{6}{\pi \Delta^{2}}\right)^{1 / 2} \exp \left(\frac{6 r^{2}}{\Delta^{2}}\right)
\end{aligned}
$$

here, $H$ is the Heaviside function and $r$ is the radial coordinate in physical space.

A comparison of filtered energy spectra for the box and the Gaussian filter is shown in Figure 2.5.

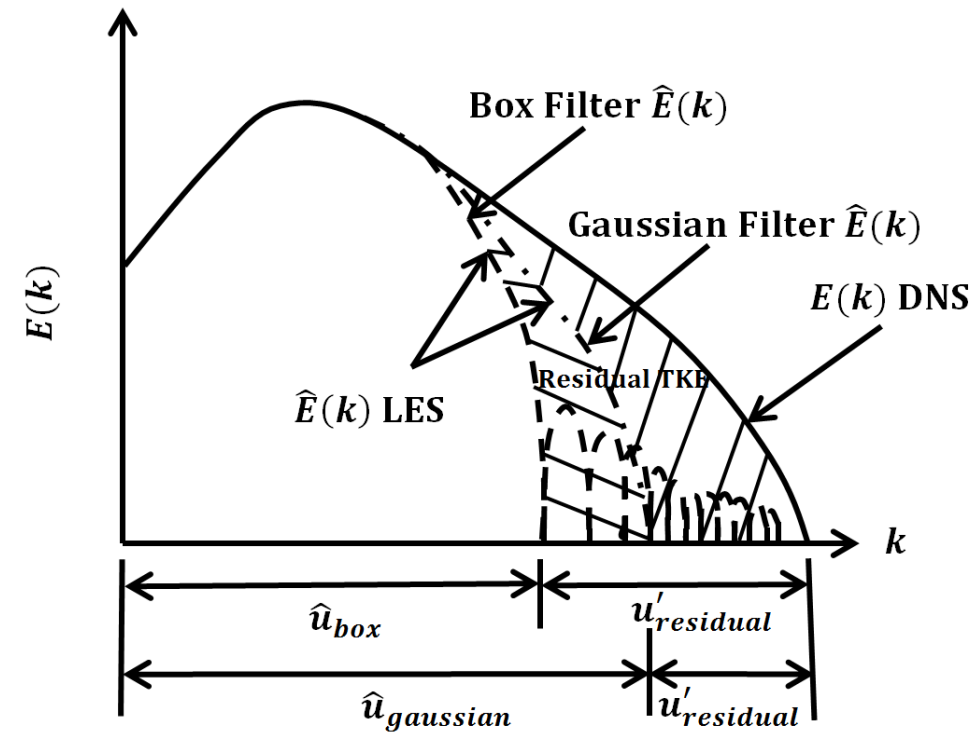

Figure 2.5. Filtered energy spectra for box and Gaussian filters 
The turbulent kinetic energy (TKE) of the residual term in LES can be expressed as,

$$
\frac{1}{2} \tau_{i i}^{\text {Residual }}=\frac{1}{2} \tau_{i i}^{R}=T K E=k_{\text {Residual }}=\int_{0}^{\infty}\left[1-\widehat{G}(\kappa)^{2}\right] E(\kappa) d \kappa
$$

From the filtered Navier-Stokes equation the turbulence stress for the resolved and residual parts can be written as,

$$
\begin{gathered}
\tau_{i j}^{\text {Resolved }}=\widehat{u}_{\imath} \widehat{u}_{J}-\overline{\hat{u}}_{i} \overline{\hat{u}}_{j} \\
\tau_{i j}^{\text {Residual }}=\overline{\hat{u}_{\imath} \hat{u}_{J}}-\overline{\hat{u}}_{i} \overline{\hat{u}}_{j}=\tau_{i j}^{R}
\end{gathered}
$$

Figure 2.5 represents the energy spectra comparison between the box and the Gaussian filtered LES as well as with DNS. The energy spectrum curve of LES is divided into two parts; the resolved portion and the residual portion. The resolved as well as the residual portion of the box and the Gaussian filtered LES are represented in terms of the turbulent kinetic energy $(k)$. Now, the anisotropic stress tensor for the actual residual part is,

$$
\tau_{i j}^{r}=\tau_{i j}^{R}-\frac{2}{3} k_{r} \delta_{i j}
$$

Here, $\tau^{r}$ represents the deviatoric stress tensor and $\tau^{R}$ represents the residual stress tensor respectively, $k_{r}$ is the turbulent kinetic energy for the residual part, $k_{r}=\frac{1}{2} \tau_{i i}^{R}$, and $\delta_{i j}$ is the Kronecker delta included in residual part of the shear stress.

The transfer of the kinetic energy between filtered velocity field and the residual motions can be expressed as follows,

The above equation can be decomposed into,

$$
\hat{E}=\frac{1}{2} \overline{\hat{u}_{\imath} \hat{u}_{\jmath}}
$$

$$
\widehat{E}=E_{f}+k_{\text {residual }}
$$

Here, $E_{f}$ is the filtered kinetic energy from the filtered velocity field and $k_{\text {residual }}$ is the residual turbulent kinetic energy obtained from the residual motions of the velocity field. The filtered and the residual kinetic energy can be expressed as follows, 


$$
\begin{gathered}
E_{f}=\frac{1}{2} \overline{\hat{u}}_{i} \overline{\hat{u}}_{i} \\
k_{r}=\frac{1}{2} \tau_{i i}^{R}=\frac{1}{2}\left(\overline{\hat{u}_{\imath} \hat{u}_{\imath}}-\overline{\hat{u}}_{i} \overline{\hat{u}}_{i}\right)
\end{gathered}
$$

The filtered kinetic energy $E_{f}$ is obtained from the conservation of energy and can be expressed as (Pope 2000),

$$
\frac{\widehat{D} E_{f}}{\widehat{D} t}-\frac{\partial}{\partial x_{i}}\left[\hat{u}_{j}\left(v \hat{S}_{i j}-\tau_{i j}^{r}-\frac{\hat{p}}{\rho} \delta_{i j}\right)\right]=-\varepsilon_{f}-P_{r}
$$

where, $\hat{S}_{i j}$ is the filtered strain rate that can be expressed as

$$
\hat{S}_{i j}=\frac{1}{2}\left(\frac{\partial \hat{u}_{i}}{\partial x_{j}}+\frac{\partial \hat{u}_{j}}{\partial x_{i}}\right)
$$

From the conservation of energy, the viscous dissipation from the filtered field will be,

$$
\varepsilon_{f}=2 v \hat{S}_{i j} \hat{S}_{i j}
$$

And the production rate from the residual kinetic energy is,

$$
\begin{gathered}
P_{r}=-\tau_{i j}^{r} \hat{S}_{i j} \\
P_{r}=2 v_{\text {Residual }} \hat{S}_{i j} \hat{S}_{i j}
\end{gathered}
$$

where, the residual shear stress $\tau_{i j}^{r}$ (Pope 2000) can be expressed as,

$$
\tau_{i j}^{r}=-2 v_{\text {Residual }} \hat{S}_{i j}
$$

Production rate $\left(P_{r}\right)$ acts as a sink for $E_{f}$ and as a source for $k_{r}$. The residual viscosity (Pope 2000) is accounted for by the Smagorinsky model,

$$
v_{\text {Residual }}=l_{s}{ }^{2}|\hat{s}|=\left(C_{s} \Delta\right)^{2}|\hat{s}|
$$

Here, $l_{s}$ is the Smagorinsky lengthscale which is analogous to mixing length and equivalent to $C_{s} \Delta$. In addition, for the filtered pressure term, the residual part of the turbulent kinetic energy is included in it, 


$$
\hat{p}=\bar{p}+\frac{2}{3} \rho k_{r}
$$

The filtered momentum equation is obtained from Eq 2.21 by substituting Eqs 2.22-28 into it

$$
\frac{\widehat{D} \hat{u}_{j}}{\widehat{D} t}=v \frac{\partial^{2} \hat{u}_{j}}{\partial x_{i} \partial x_{j}}-\frac{\partial \tau_{i j}^{r}}{\partial x_{i}}-\frac{1}{\rho} \frac{\partial \hat{p}}{\partial x_{j}}
$$

After mathematical manipulation the momentum equation (Pope 2000) can be written as,

$$
\frac{\partial \hat{u}_{j}}{\partial t}+\hat{u}_{i} \frac{\partial \hat{u}_{j}}{\partial x_{i}}=-\frac{1}{\rho} \frac{\partial \hat{p}}{\partial x_{j}}+\frac{\partial}{\partial x_{i}}\left[\left(v+v_{\text {Residual }}\right) \frac{\partial \hat{u}_{j}}{\partial x_{j}}\right]
$$

where, $v+v_{\text {Residual }}$ is the effective viscosity, and contains the kinematic viscosity $v$ and residual SGS viscosity $v_{\text {Residual }}$.

Now a question arises, does the standard Smagorinsky closure coefficient remain constant for all types of filters, or does it vary? From the literature (Pope 2000), it is found that the value of the Smagorinsky constant $\left(C_{s}\right)$ depends on the types of filter. The recommended $C_{s}$ values for the

\begin{tabular}{|c|c|c|}
\hline \multicolumn{3}{|c|}{ Standard Smagorinsky model } \\
\hline Types of filter & Equation for $C_{s}$ value & $C_{S}$ value \\
\hline Box filter & $\begin{array}{l}\text { Equation for } C_{s}(\text { Pope } 2000) \\
\qquad C_{s}=\frac{l_{s}}{\Delta} \\
\text { Here, filter width } \Delta=V_{c}^{1 / 3}, \\
\text { cell volume } V_{c}=\delta x \delta y \delta z\end{array}$ & 0.20 \\
\hline Gaussian filter & 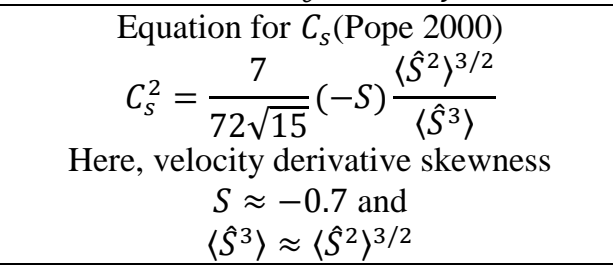 & 0.13 \\
\hline Sharp spectral filter & $\begin{array}{c}\text { Equation for } C_{s}(\text { Pope 2000) } \\
\qquad C_{s}=\frac{l_{s}}{\Delta}=\frac{1}{\pi}\left(\frac{2}{3 C}\right)^{3 / 4} \\
\text { Here, } C \text { is Kolmogorov constant, } C \approx 1.5\end{array}$ & 0.17 \\
\hline Pao filter & $\begin{array}{l}\text { Equation for } C_{S}(\text { Pope 2000) } \\
\qquad C_{S}=\frac{l_{s}}{\Delta}=\frac{\pi^{1 / 2}}{(18 C)^{3 / 4}} \\
\text { Here, } C \text { is Kolmogorov constant, } C \approx 1.5\end{array}$ & 0.15 \\
\hline
\end{tabular}
different filter types for isotropic turbulence are listed in table 2.1.

Table 2.1. Smagorinsky constant $\left(C_{s}\right)$ for different types of filter 


\subsubsection{Dynamic Smagorinsky model}

Germano et al. (1991) introduced a dynamic procedure which eliminates the necessity to specify the model coefficients at the beginning of the simulation. In this SGS model two types of filtering are involved. The first one is known as a grid filter $(\Delta)$ where the filter width is expressed as a function of local grid spacing. The second one is termed as a test filter $(\breve{\Delta})$ that needs to be larger than the grid filter. According to Germano et al. (1991), both filters are required to lie within the inertial sub-range.

The sub grid stress from the filtered Navier-Stokes equation can be expressed as,

$$
\tau_{i j}=\widehat{u_{\imath} \widehat{u}_{J}}-\hat{u}_{i} \hat{u}_{j}=-2\left(C_{S} \Delta\right)^{2}|\hat{S}| \hat{S}_{i j}
$$

After applying the test filter in the Navier-Stokes equation, the residual stress at the test scale,

$$
T_{i j}={\breve{u_{\imath}}}_{J}-\breve{\hat{u}}_{i} \breve{\hat{u}}_{j} \approx-2\left(C_{S} \breve{\Delta}\right)^{2} \mid \breve{\hat{S}}_{\mid} \breve{\hat{S}}_{i j}
$$

where, the notation $(\because)$ is adoped for the test filter and $\breve{\Delta}$ represents the test filter width which is $\breve{\Delta}>\Delta$, and the strain rate can be defined as,

$$
\breve{\hat{S}}_{i j}=\frac{1}{2}\left(\frac{\partial \breve{\hat{u}}_{i}}{\partial x_{j}}+\frac{\partial \breve{\hat{u}}_{j}}{\partial x_{i}}\right)
$$

By test filtering the sub-grid stress term $\left(\tau_{i j}\right)$ and combining it with the residual stress at a test scale, a new Leonard stress term $\left(\mathcal{L}_{i j}\right)$ can be constructed; this is also known as the 'Germano identity' and can be written as,

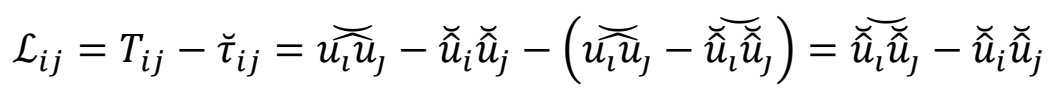

The above equation for the Leonard stress term can also be expressed as,

$$
\mathcal{L}_{i j}=2\left(C_{S} \Delta\right)^{2}\left|\widehat{\hat{S}} \widehat{S}_{i j}-2\left(C_{S} \breve{\Delta}\right)^{2}\right| \breve{\hat{S}} \mid \breve{S}_{i j}
$$

The model coefficient $C_{S}$ can be easily extracted by applying test filtering to the sub-grid stress. After taking the average of both sides of the above equation, the length scale (Lilly 1992) of the dynamic model can be expressed as, 


$$
\left(C_{S} \Delta\right)^{2}=\frac{\overline{\mathcal{L}_{l J} M_{l j}}}{\overline{M_{\imath \jmath} M_{l \jmath}}}
$$

where, $M_{i j}$ represents a model parameter, after some mathematical manipulations it can be written as,

$$
M_{i j}=2\left(\left|\widehat{\hat{S} \mid \hat{S}_{i j}}-\alpha^{2}\right| \breve{\hat{S}} \mid \breve{\hat{S}}_{i j}\right)
$$

Here, $\alpha=\breve{\Delta} / \Delta$, the test to grid filter width ratio, usually takes a value of 2 .

Here, the bar $(\cdot)$ represent spatial averaging. In addition, the value of the eddy viscosity of the dynamic eddy model can be negative. The negative values of the mixing lengthscale of the model indicate a backscatter of energy; that means that the energy due to viscous effects may bounce back from the sub-grid scale to the resolved scales. Moreover, from the literature, it is found that the dynamic Smagorinsky model may produce large negative values of $C_{S}$ which render the simulation unstable (Sagaut 2001). The 'clipping' method is the common practice for the elimination of this problem. In this method, the closure coefficient of the dynamic Smagorinsky model is set zero when the denominator is zero or the numerator $\left(\overline{\mathcal{L}_{l \jmath} M_{l \jmath}}\right)$ is negative.

\subsubsection{Deardorff model}

FDS6 incorporates the Deardorff (1973) eddy model as the default LES model. The eddy viscosity of the Deardorff model can be expressed as,

here,

$$
v_{t}=C_{v} \Delta \sqrt{k_{s g s}}
$$

$$
k_{s g s}=\frac{1}{2}\left((\overline{\hat{u}}-\hat{\hat{u}})^{2}+(\overline{\hat{v}}-\hat{v})^{2}+(\overline{\widehat{w}}-\widehat{\widehat{w}})^{2}\right)
$$

where, $\overline{\hat{u}}$ is the average value of the filtered velocity $\hat{u}$ at the grid cell centre and $\hat{\hat{u}}$ is a weighted average of $\overline{\hat{u}}$ over the adjacent cells that can be expressed as, 


$$
\begin{gathered}
\overline{\hat{u}}_{i j k}=\frac{\hat{u}_{i j k}+\hat{u}_{i-1, j k}}{2} \\
\hat{\hat{u}}_{i j k}=\frac{\overline{\hat{u}}_{i j k}}{2}+\frac{\overline{\hat{u}}_{i-1, j k}+\overline{\hat{u}}_{i+1, j k}}{4}
\end{gathered}
$$

The Deardorff model coefficient is defined as $C_{v}=0.1$.

\subsubsection{Vreman model}

Along with the other three eddy models, FDS also has the option of the Vreman model to simulate turbulent flows. To eliminate the drawback of 'backscatter' in the Smagorinsky model (Smagorinsky 1963), Vreman (2004) proposed a different kind of eddy viscosity model for LES. Vreman's eddy viscosity is expressed as,

$$
v_{t}=c \sqrt{\frac{B_{\beta}}{\alpha_{i j} \alpha_{i j}}}
$$

where,

$$
\begin{gathered}
\alpha_{i j}=\frac{\partial u_{j}}{\partial x_{i}} \\
\beta_{i j}=\Delta_{m}^{2} \alpha_{m i} \alpha_{m j} \\
B_{\beta}=\beta_{11} \beta_{22}-{\beta_{12}}^{2}+\beta_{11} \beta_{33}-\beta_{13}{ }^{2}+\beta_{22} \beta_{33}-\beta_{23}{ }^{2}
\end{gathered}
$$

Vreman's constant, $c$ is closely related to the Smagorinsky constant $C_{S}$ and can be defined as $c \approx 2.5 C_{S}$. The eddy viscosity is easy to compute as it only depends on local filter width and the first-order derivative of the velocity field. The basic concept behind the Vreman model is that the velocity field is expanded using the Taylor series, and the test filtering operation is done analytically to avoid the complexity of applying the fixed test filter as is done in the dynamic Smagorinsky model. Since the model does not require an additional fixed filtering operation, it is inexpensive compared to the dynamic Smagorinsky model for simulating turbulent flows. 
LES is a powerful tool to simulate turbulence. In the context of LES applications, the SGS eddy models must accurately model the rate of dissipation. Recent version of FDS 6.2 .0 consists these above mentioned four eddy models. Detailed review on wide variety SGS models are referred to section 1.2.3 where most of the authors adopted standards Smagorinsky model for their study as it is commonly used eddy viscosity model in practical applications and for research purposes. In the present study, we have selected the Smagorinsky eddy model to implement an explicit LES scheme based on the comparative study conducted using these four LES models which is presented in Chapter 3.

\subsection{A systematic approach to explicit scheme}

\subsubsection{Overall concept}

The main objective of this work is to develop an explicit LES scheme which fulfils LES principles and for which the numerical error converges to an acceptably small value. According to LES principles, filter widths need to lie within the inertial sub-range of the energy spectra and the scheme needs to accurately capture the energy containing eddies (Chow and Moin 2003). Kolmogorov's universal theoretical slope in the inertial sub-range (proportional to wavenumbers $\kappa^{-5 / 3}$ ), provides a criterion for identifying this range and helps to demarcate the energy containing and dissipation ranges. However, in low Reynolds number flows the universal theoretical slope is scarcely observed, but this theoretical relationship becomes more apparent as the Reynolds number increases (Pope 2000).

A systematic and sequential approach to obtaining an appropriate LES solution using explicit filtering is proposed. Firstly, a fixed filter width is determined based on a physical parameter for which an analytical expression is available in the literature. Here, the BLT is considered as a suitable parameter as it characterizes the size of large scale eddies in wall bounded flows 


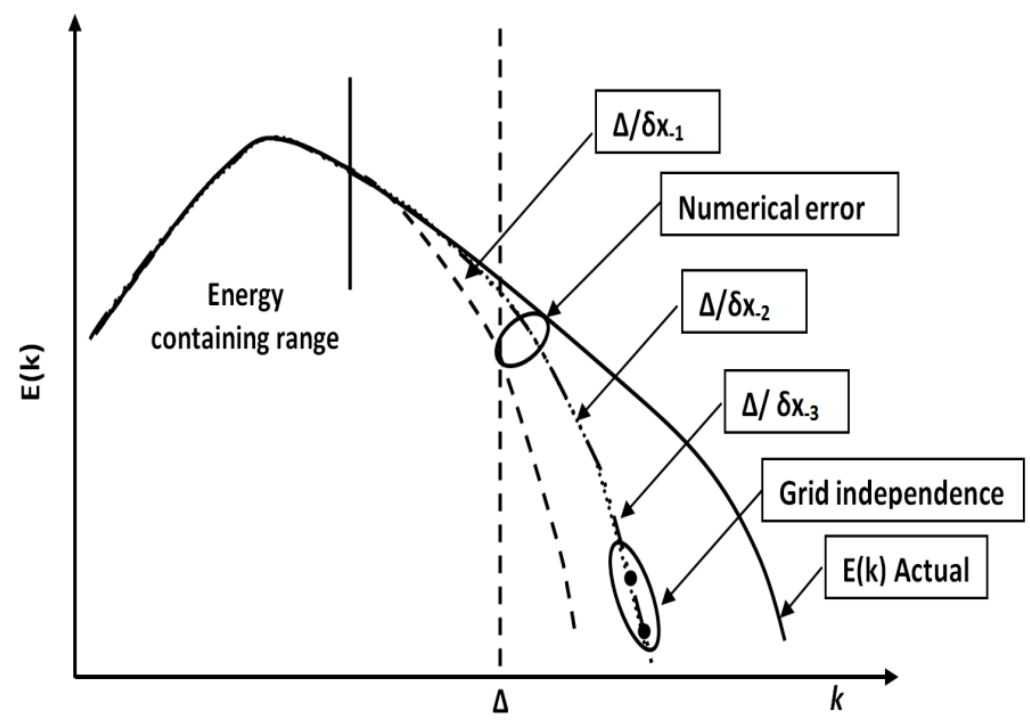

Figure 2.6. Grid convergence of a hypothetical energy spectrum with progressively larger filter to grid ratios (FGR) $\Delta / \delta x_{1}<\Delta / \delta x_{2}<\Delta / \delta x_{3}$. The dashed vertical line indicates the cut-off wavenumber for the fixed filter width $\Delta$

(Tennekes and Lumley 1972). BLT can be estimated from an analytical solution of idealized cases or empirical correlations. To reduce numerical errors (due to grid size) arising in the explicit schemes with respect to the fixed filter, the grid needs to be refined until the solution converges. A hypothetical example is shown by conducting a spectrum analysis (presented in Figures 2.6 and 2.7). In Figure 2.6 fixed filter width is chosen and the effect of reducing the grid size is investigated to obtain a grid-converged solution. The simulation is grid converged when further reductions in grid size lead to insignificant changes in the result, and the filter width remains constant. When grid-converged the example shown in Figure 2.6 effectively captures the energy containing range as the turbulence is resolved well into the inertial subrange denoted by the region of the spectrum with constant slope. A larger explicit filter than the one used in Figure 2.6 may not capture the energy containing range even when grid-converged. The effect of introducing a progressively smaller explicit filter is shown schematically in Figure 2.7. Energy spectra for four different filter widths with $\Delta_{1}>\Delta_{2}>\Delta_{3}>\Delta_{4}$ are shown, each being gridconverged. The LES $\left(\Delta_{1}\right)$ filter width lies just within the inertial sub-range but the simulation is 
unable to fully capture the energy containing eddies. Each of the three progressively smaller filter widths capture the energy containing range effectively and thus fulfils the LES principle. In this hypothetical example the spectra with filter widths $\Delta 3$ and $\Delta 4$ are the same; however, the pursuit of such filter-convergence can be elusive, computationally expensive and of minor importance. Therefore, when implementing explicit filtering a filter width should be selected which lies within the inertial sub-range and its grid-converged solution should be able to capture all energy containing scales. Although LES $\left(\Delta_{3}\right)$ and LES $\left(\Delta_{4}\right)$ may provide more accurate results than LES $\left(\Delta_{2}\right)$, the LES $\left(\Delta_{2}\right)$ results can be deemed acceptable in that it conforms to LES principles.

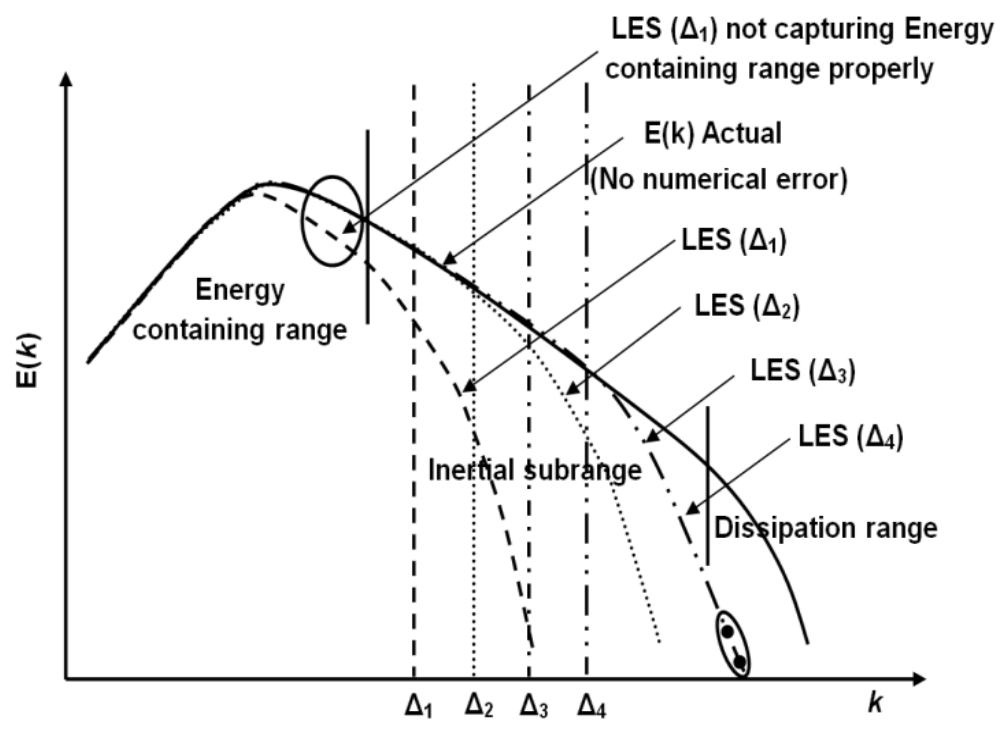

Figure 2.7. Hypothetical scenario of selection of filter width in an explicit scheme. Schematic shows the LES solution for four different filter widths with $\Delta_{1}>\Delta_{2}>\Delta_{3}>\Delta_{4}$ (all grid converged) and vertical lines are corresponding cut-off wavenumbers.

\subsubsection{Proposed explicit filter width}

In this study, the filter widths $(\Delta)$ in the explicit scheme are taken as a function of the BLT $(\delta)$. Two filter widths are selected as being $20 \%$ and $10 \%$ of the BLT, respectively. These values have been chosen according to the suggestions of Wilcox (1997) and Pope (2000) that at least ten grid points are required to accurately capture features of the boundary layer. The grid size 
$\delta x=0.1 \delta$ is therefore taken as the reference point for the coarsest grid width and the base filter width as twice that, $\Delta=2 \delta x$, which is commonly adopted in contemporary literature (Gullbrand 2002, Gullbrand and Chow 2003, Radhakrishnan and Bellan 2012, 2013 and 2015). If necessary the filter width is refined and taken as $10 \%$ of the BLT to ensure the principles of LES (i.e. filter is within the inertial subrange and energy containing eddies are fully captured) are satisfied. Although not necessary in the present modelling, in general the filter could be further reduced by half (i.e. 5\% of BLT) and so on. In the contemporary literature on implicitly filtered LES it is now common to see 20 or more grid points within the boundary layer; a situation that has been made possible by the growth in computing power. Such simulations can produce very detailed data sets of great value to the community. Here our approach is somewhat different. Our primary objective is to demonstrate a systematic approach to filter width selection in explicitly filtered LES. Accordance with LES principles is ensured but we do not attempt to produce super resolved LES, but acknowledge that higher resolution is possible and in some cases desirable.

\subsubsection{Filter width to grid spacing ratio}

As mentioned in Chapter 1, Gullbrand and Chow (2003) and Chow and Moin (2003) reported the importance of the FGR. They observed that higher order numerical schemes (fourth and sixth order), require two grids within the filter, while for second-order schemes at least four grids within the filter width are required. In the present work using a second-order code FGR values of 2, 4, 8 and 16 are used and the suitability of this choice is discussed.

\subsection{Governing equations of explicit LES scheme}

\subsubsection{Mass and momentum}

The LES equations are now rederived using an explicit filtering scheme. The instantaneous continuity and Navier-Stokes equations for variable density flow are (White 2006) 


$$
\begin{gathered}
\frac{\partial \rho}{\partial t}+\frac{\partial \rho u_{i}}{\partial x_{i}}=0 \\
\frac{\partial \rho u_{i}}{\partial t}+\frac{\partial}{\partial x_{j}}\left(\rho u_{i} u_{j}\right)=-\frac{\partial p}{\partial x_{i}}+\mu \frac{\partial}{\partial x_{j}}\left(\frac{\partial u_{i}}{\partial x_{j}}+\frac{\partial u_{j}}{\partial x_{i}}\right)+\rho g_{i}
\end{gathered}
$$

where, $u, x, \rho, p, \mu$ and $g$ denote the velocity, position coordinate, density, pressure, molecular viscosity and gravitational acceleration, respectively. The subscripts $i$ and $j$ are direction indices. In LES the governing Navier-Stokes and continuity equations are filtered in space, effectively applying a low pass filter to separate the large scale and small scale eddies (Sagaut 2001). The filtered velocity field can be defined as

$$
\hat{u}_{i}(x)=\int_{-\infty}^{\infty} \mathcal{G}\left(x-x^{\prime}, \Delta\right) u_{i}\left(x^{\prime}\right) d x^{\prime}
$$

where, $\mathcal{G}$ is the filter function and $\Delta$ is the filter width. Applying the spatial filter to the instantaneous aforementioned governing equations (2.46) and (2.47) yields

$$
\begin{gathered}
\frac{\partial \hat{\rho}}{\partial t}+\frac{\partial \widehat{\rho u_{\imath}}}{\partial x_{i}}=0 \\
\frac{\partial \widehat{\rho u_{\imath}}}{\partial t}+\frac{\partial}{\partial x_{j}}\left(\widehat{\rho u_{\imath} u_{\jmath}}\right)=-\frac{\partial \hat{p}}{\partial x_{i}}+\mu \frac{\partial}{\partial x_{j}}\left(\frac{\partial \hat{u}_{i}}{\partial x_{j}}+\frac{\partial \hat{u}_{j}}{\partial x_{i}}\right)+\hat{\rho} g_{i}
\end{gathered}
$$

In compressible flows, correlations of fluctuations of density and velocity are eliminated by applying a Favre (mass-weighted) filter defined for the velocity as $\tilde{u}_{i}=\widehat{\rho}_{\imath} / \hat{\rho}$ so that equations (2.49) and (2.50) become

$$
\begin{gathered}
\frac{\partial \hat{\rho}}{\partial t}+\frac{\partial \hat{\rho} \tilde{u}_{i}}{\partial x_{i}}=0 \\
\frac{\partial \hat{\rho} \tilde{u}_{i}}{\partial t}+\frac{\partial}{\partial x_{j}}\left(\hat{\rho} \widetilde{u_{\imath} u_{\jmath}}\right)=-\frac{\partial \hat{p}}{\partial x_{i}}+\mu \frac{\partial}{\partial x_{j}}\left(\frac{\partial \tilde{u}_{i}}{\partial x_{j}}+\frac{\partial \tilde{u}_{j}}{\partial x_{i}}\right)+\hat{\rho} g_{i} .
\end{gathered}
$$


Since the mean of the square is not equal to the square of the mean a closure model is required for the convective term in equation (2.52) involving the non-linear correlation of velocity $\widetilde{u_{\imath} u_{\jmath}}$. A subgrid-scale (SGS) stress $\tau_{i j}^{s g s}$ is introduced defined as

$$
\tau_{i j}^{s g s}=\hat{\rho}\left(\widetilde{u_{\imath} u_{\jmath}}-\tilde{u}_{i} \tilde{u}_{j}\right)
$$

and after substitution equation (2.52) becomes

$$
\frac{\partial \hat{\rho} \tilde{u}_{i}}{\partial t}+\frac{\partial}{\partial x_{j}}\left(\hat{\rho} \tilde{u}_{i} \tilde{u}_{j}\right)=-\frac{\partial \hat{p}}{\partial x_{i}}+\mu \frac{\partial}{\partial x_{j}}\left(\frac{\partial \tilde{u}_{i}}{\partial x_{j}}+\frac{\partial \tilde{u}_{j}}{\partial x_{i}}\right)-\frac{\partial \tau_{i j}^{s g s}}{\partial x_{j}}+\hat{\rho} g_{i}
$$

Traditionally the filter width is defined implicitly and directly linked to the grid spacing. In that approach, the kernel function $\mathcal{G}$ is not explicitly defined but is implicit within the discretized equations and the Favre filtered quantities in equations (2.51) and (2.54) may be obtained directly on a mesh (i.e. they are grid-based quantities) provided that a suitable SGS closure is available for $\tau_{i j}^{s g s}$. As discussed in the review presented in section 1.3.2, such a linkage entangles modelling and numerical issues, making it difficult to obtain grid independent solutions. One can reformulate the LES equations by incorporating an explicit filter that is independent of the grid. This impacts on the non-linear convective terms so that the explicitly filtered version of equation (2.54) becomes (Radhakrishnan and Bellan 2012, 2013 and 2015)

$$
\frac{\partial \hat{\rho} \tilde{u}_{i}}{\partial t}+\frac{\partial}{\partial x_{j}}\left(\hat{\hat{\rho} \tilde{u}_{\imath} \tilde{u}_{J}}\right)=-\frac{\partial \hat{p}}{\partial x_{i}}+\mu \frac{\partial}{\partial x_{j}}\left(\frac{\partial \tilde{u}_{i}}{\partial x_{j}}+\frac{\partial \tilde{u}_{j}}{\partial x_{i}}\right)-\frac{\partial \tau_{i j}^{\xi}}{\partial x_{j}}+\hat{\rho} g_{i}
$$

where the new quantity $\tau_{i j}^{\xi}$ is the explicitly filtered SGS term

$$
\tau_{i j}^{\xi}={\widehat{\rho u_{\imath} u_{j}}}_{-\widehat{\rho} \tilde{u}_{\imath} \tilde{u}_{j}}
$$

where the overhat denotes an explicitly filtered quantity, while the tilde now denotes grid-based (or implicitly filtered) quantities. According to Lund (1997), if the explicit filter width is larger 
than the grid spacing the filter will damp fluid motions at scales which are smaller than the filter width but are captured by the grid. It thus ensures that the definition of the resolved length scales of fluid motions do not vary with the refinement of the grid.

\subsubsection{Sub-grid closure}

The filtered Navier-Stokes equations require closure as the sub-grid stress tensor cannot be expressed in terms of resolved scales (Gullbrand 2002). In this study, a damped standard Smagorinsky model accounts for the effect of the small scale eddies on the resolved scales. Other models, such as the dynamic Smagorinsky model (DSM), dynamic mixed model (DMM) and dynamic reconstruction model (DRM) (Gullbrand and Chow 2003) are commonly reported in the literature.

In conventional LES that uses an implicit filtering scheme the standard Smagorinsky (1963) model SGS stresses are modelled as

$$
\tau_{i j}^{s g s}=-2 \hat{\rho} v_{t} \tilde{S}_{i j}
$$

where the eddy viscosity may be defined as

$$
\begin{gathered}
v_{t}=\left(C_{S} \Delta\right)^{2}|\tilde{S}| \\
|\tilde{S}| \equiv\left(2 \tilde{S}_{i j} \tilde{S}_{i j}\right)^{1 / 2}, \quad \tilde{S}_{i j}=\frac{1}{2}\left(\frac{\partial \tilde{u}_{i}}{\partial x_{j}}+\frac{\partial \tilde{u}_{j}}{\partial x_{i}}\right)
\end{gathered}
$$

where $C_{S}$ is a constant model coefficient, $\Delta=(\delta x \delta y \delta z)^{1 / 3}$ is taken as the implicit (grid-based)

filter width, $|\tilde{S}|$ is the magnitude of the strain rate and, repeating to avoid ambiguity, $\tilde{u}_{i}$ is the resolved scale velocity on the grid. On the other hand, the explicit LES SGS stresses can be modelled as

$$
\tau_{i j}^{\xi}=-2 \hat{\rho} v_{t} \hat{\tilde{S}}_{i j}
$$

where the eddy viscosity of the explicit LES scheme may be obtained as 


$$
\begin{gathered}
v_{t}=\left(C_{S} \widehat{\Delta}\right)^{2}|\hat{\tilde{S}}| \\
|\hat{\tilde{S}}|=\left(2 \hat{\tilde{S}}_{i j} \hat{\tilde{S}}_{i j}\right)^{1 / 2}, \quad \hat{S}_{i j}=\frac{1}{2}\left(\frac{\partial \hat{\tilde{u}}_{i}}{\partial x_{j}}+\frac{\partial \hat{\tilde{u}}_{j}}{\partial x_{i}}\right)
\end{gathered}
$$

where the explicit filter width $\widehat{\Delta}$ is independently specified but larger than the grid size (here it is taken as a function of the BLT), $\widehat{\tilde{u}}$ is the explicitly filtered velocity obtained from the grid velocities (see section 2.4.4 below).

\subsubsection{Damping function for model coefficient $C_{S}$}

Turbulence is damped in proximity to solid walls. This is accounted for by replacing the Smagorinsky constant, $C_{S}$, with the Van Driest damping (Van Driest 1956) function $C_{S_{-} D a m p}$, given by

$$
C_{S_{-} \text {Damp }}=C_{S 1}\left[1-e^{-y^{+} / A^{+}}\right]
$$

where, $A^{+}=26$ and model coefficients $C_{S 1}=0.2$. The non-dimensional distance normal to the

wall is defined as $y^{+}=y u_{\tau} / v$ where $u_{\tau}$ is the friction velocity calculated as $u_{\tau}=\sqrt{\tau_{w} / \rho}$; $\tau_{w}$ is the local shear stress at the wall. The value $C_{S_{-} \text {Damp }}$ replaces the value of $C_{S}$ in the constant Smagorinsky model.

\subsubsection{Filter functions}

In this study, the filtered velocity in the explicit LES scheme is obtained using a onedimensional filter function following the method developed by Lund (1997). The general formulation of the filter and weighting function can be expressed as

$$
\begin{gathered}
\hat{\tilde{u}}_{i}=\sum_{j=-(n-1) / 2}^{(n-1) / 2} w_{j} \tilde{u}_{i+j} \\
\sum_{j=-(n-1) / 2}^{(n-1) / 2} w_{j}=1
\end{gathered}
$$


where $j$ is the cell index, $n$ is the number of grid points (or nodes) within the filter and $w_{j}$ represents the weighting factors given to the velocities in each node. Weighting factors are defined such that at the central grid point $w_{0}=\frac{1}{2}$, and the remaining half is equally distributed among neighbouring grid points.

Half to the central grid point is applied as the trapezoidal rule is used. In effective filter width $\Delta=2 d x$ Case, weighting $(1 / 4,1 / 2,1 / 4)$ is applied (Lund 1997) and the actual filter width is calculated as $\sqrt{6} \approx 2.44$ using the following equation,

$$
\Delta=\sqrt{12 \sum_{j=-(n-1) / 2}^{(n-1) / 2} j^{2} w_{j}} d x
$$

Therefore, in this study when filter widths of 2, 4, 8 and 16 are stated, these effectively mean $\sqrt{6}$, $\sqrt{15}, \sqrt{45}$ and $\sqrt{153}$. Effective filter width is in fact increased by 1.6-1.85 times instead of 2 . Similar procedure was adopted by Gullbrand and Chow (2003) for FGR 2 and 4.

\subsubsection{Energy equation}

Since one of the test cases we consider is a buoyancy driven flow in a differentially heated rectangular cavity, a filtered energy equation is required. The implicitly filtered (grid-based) equation for energy is

$$
\frac{\partial\left(\hat{\rho} C_{p} \tilde{T}\right)}{\partial t}+\nabla \cdot\left(\hat{\rho} \tilde{u} C_{p} \tilde{T}-\frac{\hat{\rho} \mu_{t} C_{p}}{P r_{t}} \tilde{T}\right)=\frac{\mathrm{D}(\bar{p})}{\mathrm{D} t}-\nabla \cdot \tilde{q}^{\prime \prime}
$$

where $C_{p}$ is the specific heat at constant pressure, $\tilde{T}$ is the Favre filtered temperature, $\bar{p}$ is the Reynolds filtered thermodynamic pressure based on the ideal gas equation of state

$$
\frac{\bar{p}}{\widehat{\rho}}=\frac{R}{\bar{W}} \tilde{T}
$$


where $R$ is the universal gas constant and $\bar{W}$ is the Reynolds filtered molecular weight (here taken as the value for standard air), and $\tilde{q}^{\prime \prime}$ is a source representing the resolved conductive, diffusive and radiative heat fluxes. Note that grid based filtering of equation 2.67 suffices because the subgrid thermal diffusivity is modelled from the subgrid viscosity and the turbulent Prandtl number as shown in the second term inside brackets. The subgrid viscosity is closed with an explicit filter according to equation 2.61.

\subsubsection{Pressure equation}

In this study, pressure is calculated by solving the Poisson equation given in equations (2.69-70).

$$
\begin{gathered}
\nabla^{2} H=-\frac{\partial(\nabla \cdot \tilde{u})}{\partial t}-\nabla \cdot F \\
F=-\tilde{u} \times \omega-\tilde{p} \nabla\left(\frac{1}{\hat{\rho}}\right)-\left(\frac{1}{\hat{\rho}}\right)\left[\left(\hat{\rho}-\rho_{0}\right) g+f_{b}+\nabla \cdot \tau_{i j}^{\xi}\right]
\end{gathered}
$$

here $H$ is the total pressure (also known as the stagnation energy per unit mass; $\left|\tilde{u}^{2}\right| / 2-\tilde{p} / \hat{\rho}$ ), $F$ is referred to collectively as momentum flux. $\tilde{p}$ is the perturbation pressure (which is calculated from $H$ value from the previous time step), while $\omega$ represents vorticity, $\hat{\rho}$ is the instantaneous density and $\rho_{0}$ represents density at initial temperature, $g$ is the acceleration of gravity and $f_{b}$ is the external force vector (excluding gravity), $\tau_{i j}^{\xi}$ is the explicitly filtered SGS stress tensor. The above approximation allows to have efficient solutions with fast, direct solvers optimized for uniform grids.

\subsection{Calculation of energy spectrum}

Energy spectra are a fundamental indicator of the conformance of the simulations to the principles of LES. Energy spectra $E(\kappa)$ can be computed at a single instant in time by taking kinetic energies integrated throughout the system domain and plotting them against wavenumbers $(\kappa)$ to carry out the energy spectrum analysis. Energy spectrum analysis of any turbulent flow system provides information on energy distribution among eddies with different length scales. The energy spectrum $E(\kappa)$ is obtained by calculating the energy contained in the 
wavenumber range $(0, \infty)$ through $\int_{0}^{\infty} E(\kappa) d \kappa=T K E$. The turbulent kinetic energy are calculated as $T K E=0.5\left(\overline{u^{\prime 2}}+\overline{v^{\prime 2}}+\overline{w^{\prime 2}}\right)$; where $u^{\prime}, v^{\prime}$ and $w^{\prime}$ are the velocity fluctuations along three Cartesian directions (see section 2.6.1) and the wavenumber is computed as $\kappa=$ $\sqrt{\kappa_{x}^{2}+\kappa_{y}^{2}+\kappa_{z}^{2}}$. For more details on the energy spectrum $E(\kappa)$ and its rigorous mathematical formulations, the reader is referred to the seminal text of LES (Pope 2000). A Matlab program is used to calculate the energy spectrum of test cases studied in this work.

\subsection{Defining nature of studied flows}

\subsubsection{Estimation of $\operatorname{Reynolds} \operatorname{number}\left(\operatorname{Re}_{\lambda}\right)$}

The Reynolds number is an important parameter to quantify the nature of flows and whether they are laminar or turbulent. A Taylor scale Reynolds number $\left(R e_{\lambda}\right)$ can be used to quantify the nature of the flow. The literature of Mansour et al. (1994), Sreenivasan (1994) and Djenidiet al. (2013) considered the Reynolds number $\left(R e_{\lambda}\right)$ of 50 as being of critical value. These authors considered $R e_{\lambda} \leq 50$ as a low Reynolds number flow and $R e_{\lambda}>50$ as a high Reynolds number flow. The following equation has been used to estimate the Reynolds number,

$$
R e_{\lambda}=\frac{u^{\prime} \lambda}{v}
$$

The Taylor microscale $\lambda$ in the inertial sub range can be computed using the following equation,

$$
\lambda=\left(\frac{10 v k}{\varepsilon}\right)^{1 / 2}
$$

with $k$ being the turbulent kinetic energy and $\varepsilon$ its dissipation rate. In the inertial range the eddies are expected to be isotropic (Batchelor 1953; Saffman 1967) and the turbulence intensity scales according to $u^{\prime}=(2 / 3 k)^{1 / 2}$.

\subsubsection{Estimation of Rayleigh numbers $(R a)$}

The Rayleigh number is a dimensionless number associated with buoyancy driven flow. A Rayleigh number $(R a)$ is valuable insofar as it provides information about the form of heat 
transfer such as whether conduction is dominant over convection, or vice versa. Typically it is

assumed that the flow becomes turbulent when the Rayleigh number is between $10^{8}$ and $10^{9}$ (Lau et al. 2013). It is defined as

$$
R a=\frac{g \beta}{v \alpha}\left(T_{s}-T_{\infty}\right) L^{3}
$$

where $L$ is a characteristic length, $g$ is the acceleration due to gravity, $\beta$ is the thermal volumetric expansion coefficient, $v$ and $\alpha$ is the kinematic viscosity and thermal diffusivity of the fluid and, $T_{s}$ and $T_{\infty}$ are the temperatures of the surface and the bulk of the fluid respectively. In the simulations is $R a=4.68 \times 10^{10}$ which is the same as in the experiment by Cheesewright et al. (1986).

\subsection{Solution algorithm}

The boundary conditions for the explicit LES scheme, such as velocity $\left(\tilde{u}^{n}\right)$, density $\left(\hat{\rho}^{n}\right)$, and the fluctuating stagnation energy per unit mass $\left(H^{n}\right)$ are known for a specified grid cell and at a the $n$th time step. The temperature $\left(\tilde{T}^{n}\right)$ is calculated in each grid cell using the equation of state. Furthermore, the background pressure $\left(\hat{p}^{n}\right)$ is known for given computational domain. Let the superscripts, $n, n+1$, denote discrete time values, $t^{n+1}=t^{n}+\Delta t$. The superscript $(*)$ denotes a predicted value at some intermediate $t^{n}<t^{*}<t^{n+1}$. The governing equations are solved using an explicit second-order predictor/corrector scheme is given as follows:

\section{Predictor}

Step 1. transport and equation of state

At the following time step the density $\left(\hat{\rho}^{*}\right)$ is calculated using an explicit Euler method. The density is calculated by the following procedure,

$$
\hat{\rho}^{*}=\hat{\rho}^{n}+\Delta t\left[\nabla \cdot \hat{\rho}^{n} \tilde{u}^{n}\right]
$$

The asterisk denotes a first order accurate estimate at the next time step.

The thermodynamic pressure $\left(\hat{p}^{*}\right)$ which is link to equation of sate is calculated as 


$$
\hat{p}^{*}=\hat{p}^{n}+\Delta t\left(\frac{\partial \hat{p}}{\partial t}\right)^{n}
$$

The temperature $\left(\tilde{T}^{*}\right)$ are computed using the equation of state.

$$
\tilde{T}^{*}=\frac{\widehat{p}^{*} \bar{W}}{\widehat{\rho}^{*} R}
$$

Step 2. compute the divergence

The divergence $(\nabla \cdot \tilde{u})^{*}$ is calculated from the equation (2.67) using the above estimated thermodynamic quantities. Here the parentheses is used to donate that the velocity vector $\left(\tilde{u}^{*}\right)$ has not yet been computed at the following time step, only its divergence is computed.

Step 3. solve the Poisson equation

The poisson equation is solved for the pressure term at the previous time level

Step 4. update the velocity field

$$
\nabla^{2} H^{n}=-\frac{(\nabla \cdot \tilde{u})^{*}-\nabla \cdot \tilde{u}^{n}}{\Delta t}-\nabla \cdot F^{n}
$$

The velocity is calculated at the intermediate time level using the following equation;

$$
\tilde{u}^{*}=\tilde{u}^{n}+\Delta t\left[F^{n}+\nabla H^{n}\right]
$$

Note that this procedure guarantees that the divergence of the estimated velocity field, $\nabla \cdot \tilde{u}^{*}$, is identically equal to the divergence that is derived from the estimated thermodynamic quantities, $(\nabla \cdot \tilde{u})^{*}$, in Step 2.

Step 5. Checking the CFL stability condition for the time step

$$
C F L=\Delta t \max \left(\frac{\tilde{u}_{i}}{\delta x_{i}}\right)<1
$$

If the CFL condition is not satisfied the timestep is reduced and the procedure returns to step 1 (Transport and equation of state). If the CFL condition is satisfied, the procedure continues to the corrector step.

\section{Corrector}

Step 1. transport and equation of state for intermediate temperatre 
The density is corrected at the next time step;

$$
\hat{\rho}^{n+1}=\frac{1}{2}\left(\hat{\rho}^{*}+\hat{\rho}^{n}\right)-\frac{1}{\Delta t}\left[\nabla \cdot \hat{\rho}^{*} \tilde{u}^{*}\right]
$$

The background pressure is also corrected in the same way

$$
\hat{p}^{n+1}=\hat{p}^{*}+\Delta t\left[\left(\frac{\partial \hat{p}}{\partial t}\right)^{*}+\left(\frac{\partial \hat{p}}{\partial t}\right)^{n}\right]
$$

The intermadiate temperature $\left(\tilde{T}^{*}\right)$ are calculated from the equation of state.

$$
\tilde{T}^{*}=\frac{\widehat{p}^{n+1} \bar{W}^{*}}{\widehat{\rho}^{n+1} R}
$$

Step 2. update the temperature field

The final temperature $\left(\tilde{T}^{n+1}\right)$ is calculated using the equation of state considering the updated density and composition.

$$
\tilde{T}^{n+1}=\frac{\widehat{p}^{n+1} \bar{W}^{n+1}}{\hat{\rho}^{n+1} R}
$$

Step 3. compute the divergence

The divergence $(\nabla . \tilde{u})^{n+1}$ is calculated using the corrected thermodynamic quantites from the energy equation.

Step 4. solve the Poisson equation

The pressure is calculated using the following equation

$$
\nabla^{2} H^{*}=-\left[\frac{(\nabla \cdot \tilde{u})^{n+1}-\frac{1}{2}\left((\nabla \cdot \tilde{u})^{*}+(\nabla \cdot \tilde{u})^{n}\right)}{\Delta t / 2}-\nabla \cdot F^{*}\right]
$$

Step 5. update the velocity field

The velocity is corrected at the following time step,

$$
\tilde{u}^{n+1}=\frac{1}{2}\left(\tilde{u}^{*}+\tilde{u}^{n}\right)-\frac{1}{\Delta t}\left[F^{*}+\nabla H^{*}\right]
$$

Note again that the divergence of the corrected velocity field is identically equal to the divergence that was computed in Step 3. 


\subsection{Conclusions}

In LES, sub-grid scales (SGS) or eddy viscosity models play an important role predicting turbulent flows accurately. A recent version of FDS 6.2 .0 contains four eddy models, namely a standard Smagorinsky model, a dynamic Smagorinsky model, and the Deardorff and Vreman models. Mathematical formulations of these models to compute eddy viscosity to account for turbulence have been reviewed in this chapter. Subsequently, a proposed overall concept of simulating wall-bounded turbulent flows, a selection of filter width as a function of the physical parameter of fluid flow such as boundary layer thickness (BLT), the importance of a filter to grid spacing ratio (FGR) in explicit LES schemes and the solution procedure of the governing equations have been reported. 


\section{Chapter 3}

\section{Assessment of baseline code}

\subsection{Introduction}

The development of FDS was motivated by the need to simulate building-fires. It is a CFD based fire model initially developed to simulate low speed thermal buoyancy driven flows, namely flows of smoke and hot gases generated by fires. Subsequently, pyrolysis and combustion submodels have been added making it a state of the art fire model. FDS is an open source code which employs a second-order numerical scheme and it incorporates an LES model that is invoked by default. The version, FDS 6.2.0, offers the user a choice of four SGS models, namely the Standard Smagorinsky, the dynamic Smagorinsky, the Deardorff and the Vreman models. As all of these are implicitly filtered schemes, it poses challenges to the adequacy and efficiency of these SGS models and their sensitivity to grids. The FDS source code (more specifically its standard Smagorinsky model) is chosen for implementing the explicit LES scheme described in Chapter 2. The reason for nominating this SGS model for conversion to an explicit LES scheme is demonstrated in this chapter. In this chapter, the grid sensitivity of FDS' SGS models has been studied. In the process, the adequacy of the second-order numerical scheme (accurate in time and space) has also been assessed. A well-defined problem is studied namely turbulent flow over a backward facing step to compare the predicted flow fields with published experimental results. Those presented by Jovic and Driver (1994) for flow over a backward facing step are used for evaluating the various sub-grid scale models.

The simulation of flow over a backward facing step was chosen for its simple geometry, but it contains relatively complex flow physics. This configuration is well suited to study the fluid flow behaviour that display separation, recirculation and reattachment phenomena, which have 
important implication in many practical and engineering applications such as in internal flow systems like diffusers, combustors and channels with sudden expansions, and in external flows such as those around aerofoils and building (Le, Moin and Kim 1997). Moreover, a plethora of well-established numerical and experimental results are available in the literature (Kuehn 1980, Isomoto and Honami 1989, Neto, Metias and Lesieur 1993, Jovic and Driver 1994, Le, Moin and Kim 1997) for the case studied.

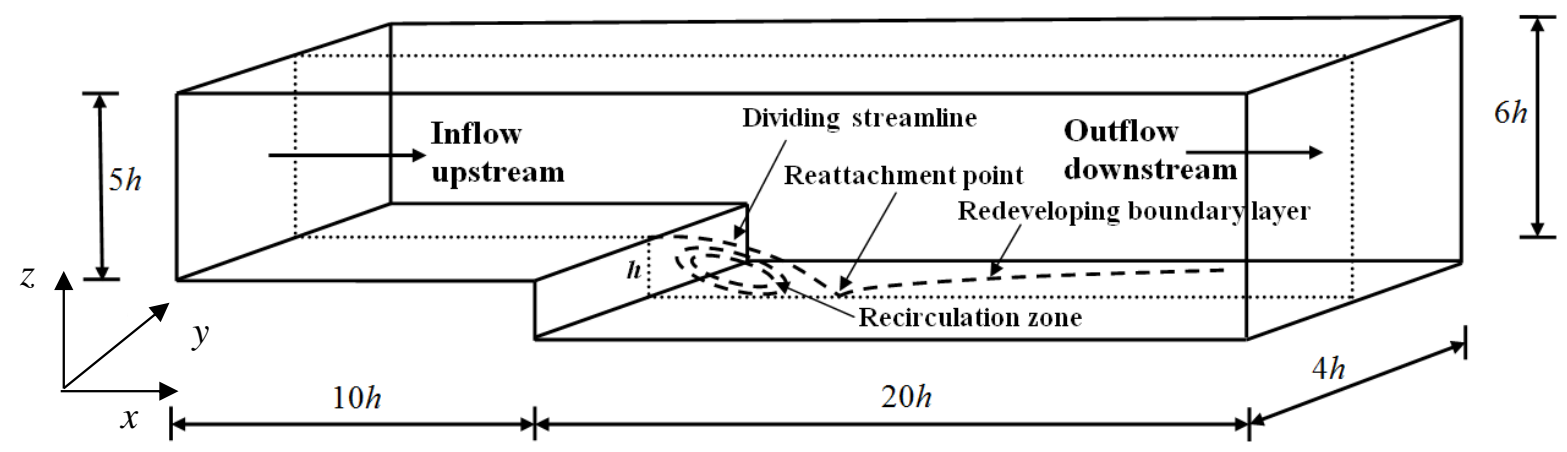

Figure 3.1. Schematic view of backward facing step flow configuration (not to scale)

\subsection{Numerical simulation}

The computational domain of the numerical simulation of the backward facing step is set up exactly according to the well-established numerical study of Le Moin and Kim (1997). According to their study, it is found that the configuration under consideration is sufficient for capturing all the prominent flow features of turbulent flow over a backward facing step as also in the experiment conducted by Jovic and Driver (1992) and requires less computational resources.

Figure 3.1 shows a schematic of the configuration, indicating the dividing streamline and recirculation zone that forms as the fluid flows over the step. It also shows the reattachment zone and redevelopment of the boundary layer after the fluid passes the reattachment zone downstream of the step. These complex flow features render it a useful and demanding benchmark case for validation of LES schemes. 
The fluid is air at a temperature of $20^{\circ} \mathrm{C}$, and the kinematic viscosity $(v)$ and density $(\rho)$ are taken to be $1.53 \times 10^{-5} \mathrm{~m}^{2} / \mathrm{s}$ and $1.2 \mathrm{~kg} / \mathrm{m}^{3}$, respectively. The step height $(h)$ is $9.6 \times 10^{-3} \mathrm{~m}$. The streamwise length of the upstream portion is $10 \mathrm{~h}$ and the length of the downstream outflow section is $20 h$. The flow width is $4 h$. The height of the upstream and downstream sections are $5 h$ and $6 h$, respectively, corresponding to an expansion ratio of 1.2 and calculated as $E R=L_{z} /\left(L_{z}-h\right)$. The Reynolds number $\left(R e_{h}=h U_{0} / v\right)$ is 5100 based on the step height $h$ and the reference velocity $U_{0}$. In the experiment the reference flow speed $\left(U_{0}\right)$ was maintained at $7.72 \pm 0.03 \mathrm{~m} / \mathrm{s}$ at section $3 h$ upstream of the step. The difficulty to obtain desired reference velocity in the numerical simulation is well known. In this study, the overall velocity is maintained $7.96 \mathrm{~m} / \mathrm{s}$ for different viscosity models which is almost $2.5 \%$ higher than ideally desired at location $3 h$ upstream of the step. The streamwise velocities are normalized using the upstream free stream reference velocity $U_{0}$. The reference velocity fluctuation for different viscosity models is maintained within $\pm 0.02 \mathrm{~m} / \mathrm{s}$. Mean velocities and turbulence statistics are analysed at two different stations $(x / h=4$ and $x / h=6)$ along the streamwise direction. The dimensionless mean $\left(u / U_{o}\right)$ velocity components and turbulence intensities or Reynolds stresses $\left(\overline{u^{\prime} u^{\prime}}\right)$ normalized by the reference velocity $U_{0}^{2}$ are compared against the vertical axis $(z / h)$ in order to obtain agreement between the simulated and experimental data. Generally, energy is transferred from the largest eddies to the smallest ones on a timescale of about one large eddy turnover. In this flow case, one recirculation time $\left(t_{E}\right)$ near the step height $(h)$ is calculated as $5 \times 10^{-3} s$ which is considered as unit eddy turnover time. The flow achieved statistically steady state at approximately $800 t_{E}$. After an initial run of $800 t_{E}$, the flow velocity is averaged over the time for another $2000 t_{E}$. The average velocity data was obtained at $0.01 \mathrm{~s}$ interval (that means nearly two eddy turnover time) and statistical average of 1000 data points was taken which is considered adequate.

Three uniform computational resolutions namely coarse, medium and fine, are used along the streamwise $\left(N_{x}\right)$, spanwise $\left(N_{y}\right)$ and vertical $\left(N_{z}\right)$ directions. The grid resolved quantities are 
physically interpreted as cell means. In implicit LES, the filter width is taken as the cube root of the cell volume, $\Delta=V_{c}^{1 / 3}$, the cell volume $V_{c}=\delta x \delta y \delta z$; where $\delta x, \delta y$ and $\delta z$ are the grid spacings along the three directions of the Cartesian coordinate system. The filter width to grid spacing $(\Delta / \delta x)$ is considered as one since the filter width $(\Delta)$ is equivalent to the grid spacing $(\delta x)$. For the grid sensitivity study, instead of random grid selection, for the convenience grids are refined in terms of the step height to grid spacing ratios $(h / \delta z)$ in this study. This grid selection process is adopted from the LES study of flow over a backward facing step by Panjwani (2010) and Toms (2015). The step height to grid spacing ratios are considered as $h / \delta z=3,5$ and 10. Table 3.1 presents the selected grid resolutions, considered model coefficients of SGS models implemented in the numerical simulation along with the measured wall unit (see section 2.4.2) $n_{\text {min }}^{+}$values which represents the minimum grid spacing in the wallnormal direction at the outflow test section. It can be observed that despite the same grid resolution, resolution in terms of nominal minimum wall can be different.

Table 3.1. Considered model coefficients and estimated wall unit $\left(n^{+}\right)$values of SGS models

\begin{tabular}{|c|c|c|c|c|c|}
\hline \multicolumn{2}{|c|}{ SGS models } & Smagorinsky & Dynamic Smagorinsky & Deardorff & Vreman \\
\hline Model coefficients $\left(\boldsymbol{C}_{*}\right)$ & 0.20 & -- & 0.10 & 0.07 \\
\hline Grid & $N_{x} \times N_{y} \times N_{z}$ & $n_{\text {Wall }}^{+}$ & $n_{\text {Wall }}^{+}$ & $n_{\text {Wall }}^{+}$ & $n_{\text {Wall }}^{+}$ \\
\hline Coarse $(\mathrm{C})$ & $90 \times 40 \times 60$ & $\leq 3.80$ & $\leq 3.98$ & $\leq 4.34$ & $\leq 4.45$ \\
\hline Medium $(\mathrm{M})$ & $150 \times 60 \times 100$ & $\leq 2.17$ & $\leq 2.32$ & $\leq 2.56$ & $\leq 2.71$ \\
\hline Fine $(\mathrm{F})$ & $300 \times 120 \times 200$ & $\leq 0.83$ & $\leq 1.02$ & $\leq 1.17$ & $\leq 1.28$ \\
\hline
\end{tabular}

It is to be noted that in the DNS study of Le, Moin \& Kim (1997) the wall unit ranged from $n_{\min }^{+} \approx 0.3$ to $n_{\max }^{+} \approx 31$. Furthermore, while Akselvoll and Moin (1993) performed a LES of backward facing step with $R e_{h}=5100$ (based on the step height, $h$ ) which is similar to this study, they refined the resolution to a nominal wall unit, $n^{+}=0.8$.

\subsection{Boundary conditions}

Boundary conditions play an important role in any numerical simulations and this is particularly true for LES to obtain appropriate solution. Inlet boundary conditions are important as in most of 
the cases the downstream flow development is largely influenced by the inlet flow behavior. The boundary conditions for the backward facing step simulations are as follows. In figure 3.1, at the inflow on the left end of the domain a constant and uniform atmospheric pressure is applied. At the outlet on the right end of the domain gradients of pressure and velocity are prescribed as zero. The lower boundary is a solid wall with the near wall behaviour modelled by the WernerWengle approximation to the log-law (Werner and Wengle 1991). Zero pressure gradient and a no-slip boundary condition is applied at the walls (side and bottom) while at the upper boundary the vertical velocity is $w=0$ while streamwise and vertical velocity gradients are $\partial u / \partial z=$ $\partial w / \partial z=0$ which is consistent with Le, Moin and Kim (1997). The turbulence was initialized by some random perturbation.

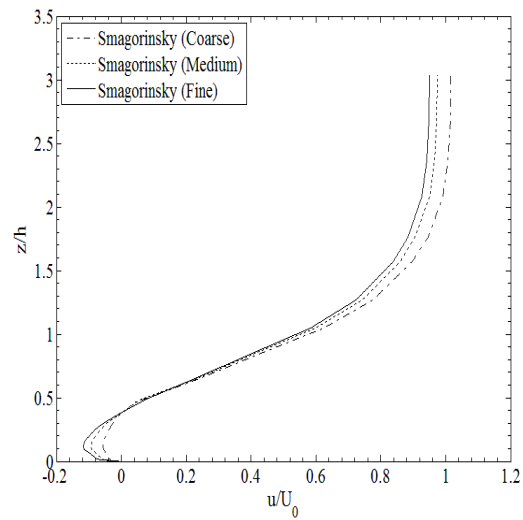

(a) Constant Smagorinsky

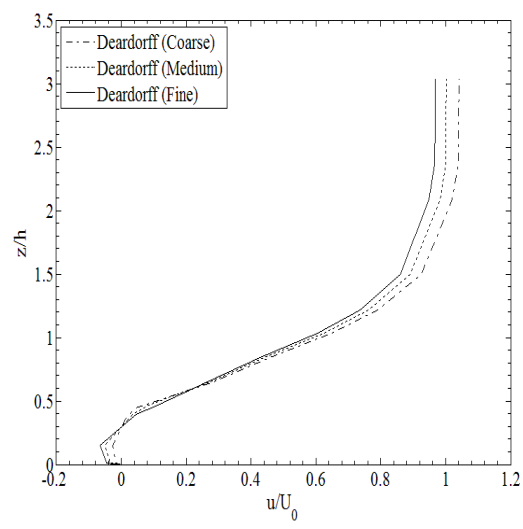

(c) Deardorff

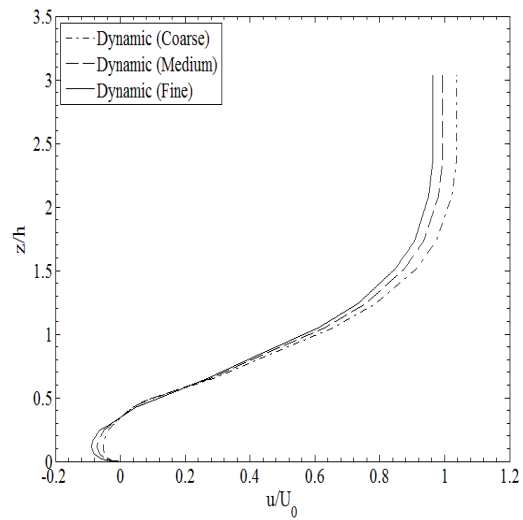

(b) Dynamic Smagorinsky

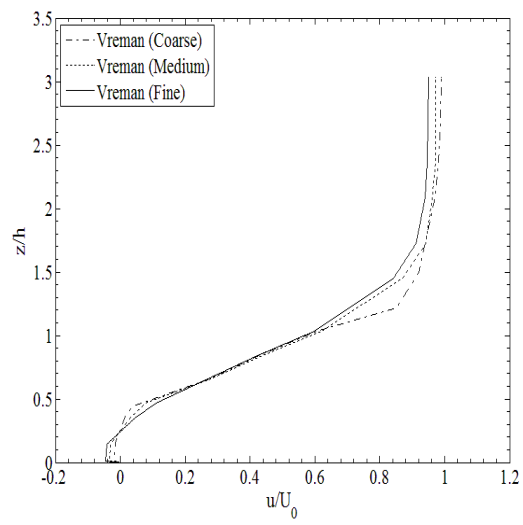

(d) Vreman

Figure 3.2. Mean velocity profiles at test section $x / h=4$ for the SGS models 


\subsection{Results and discussions - grid sensitivity}

With the implicitly filtered SGS models (the standard Smagorinsky, the dynamic Smagorinsky, the Deardorff and the Vreman SGS models), simulations of flow over a backward facing step were performed for the three grid resolutions (coarse, medium and fine) as shown in Table 3.1. All the simulations were carried out to examine the influence of refinement of grids on their solutions. The simulated results are analysed based on the mean velocity and the turbulence intensity. Results are compared at two different test locations $x / h=4$ and 6 . The grid sensitivity is analysed both graphically and quantitatively. The main purpose of this analysis is to identify the most promising SGS model among the four for the conversion to an explicit LES model.

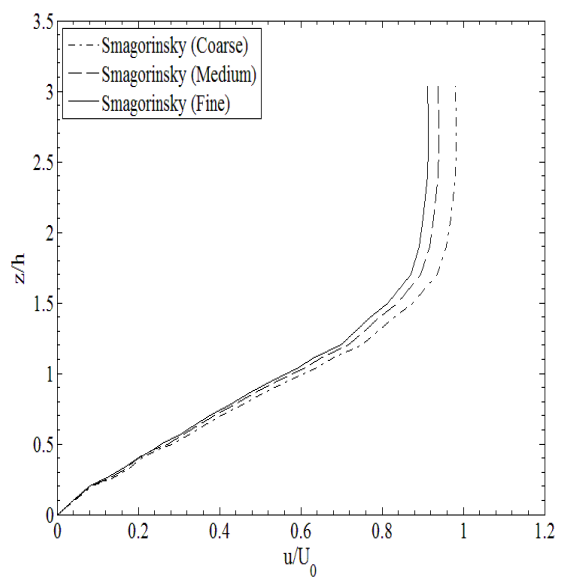

(a) Constant Smagorinsky

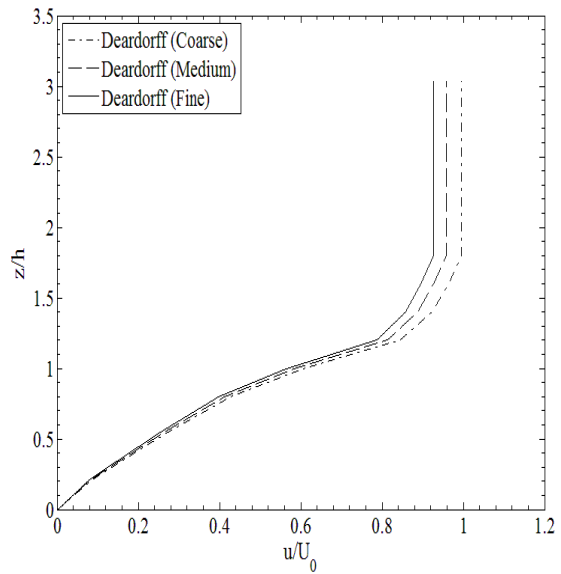

(c) Deardorff

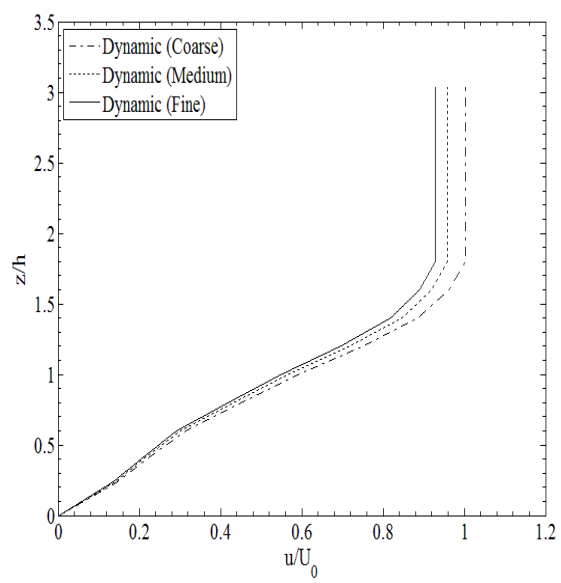

(b) Dynamic Smagorinsky

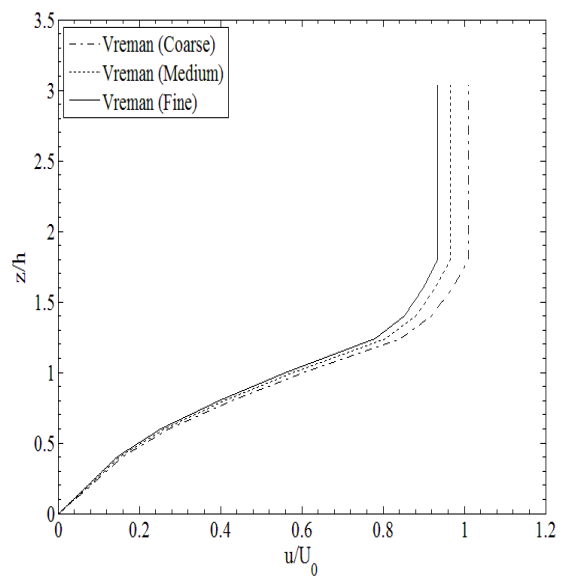

(d) Vreman

Figure 3.3. Mean velocity profiles at test section $x / h=6$ for the SGS models 


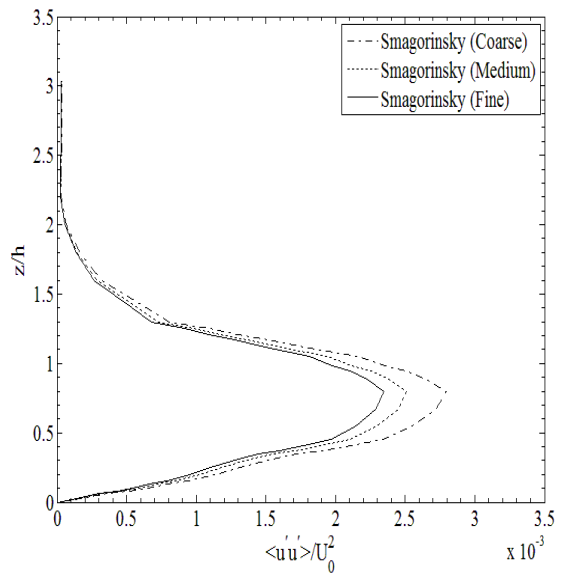

(a) Constant Smagorinsky

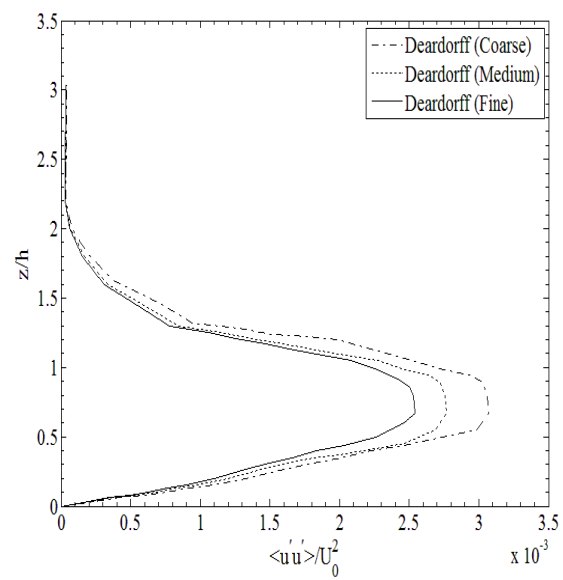

(c) Deardorff

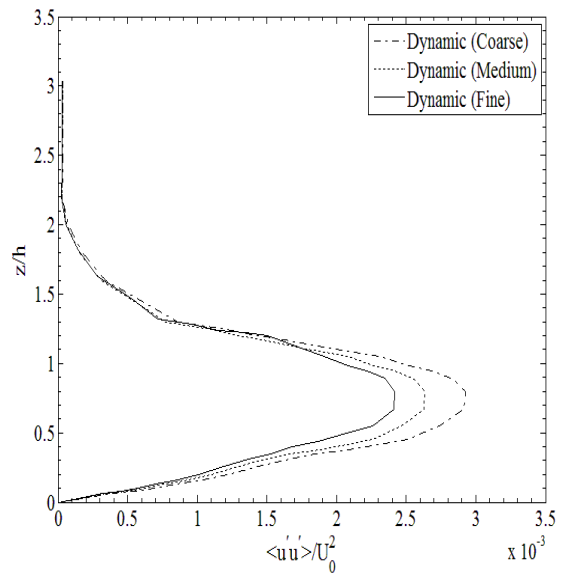

(b) Dynamic Smagorinsky

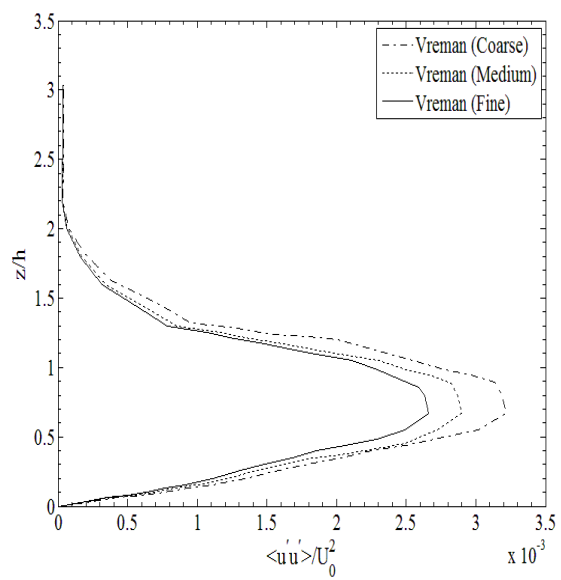

(d) Vreman

Figure 3.4. Turbulence intensities at test section $x / h=4$ for the SGS models

\subsubsection{Graphical comparison -mean velocity and turbulence intensity}

Figures 3.2 and 3.3 present the comparison of the mean velocity profiles from simulations with four SGS models at stations $x / h=4$ and $x / h=6$. This parameter was averaged in time and non-dimensionalised with the free stream reference velocity $U_{0}$. As shown in Table 3.1, at fine grid resolutions, the wall units $\left(n_{\min }^{+}\right)$along the vertical direction are reported in the range of 0.8 to 1.3 for all the four models. It also appears from the figures that the tendency of numerical convergence of the standard Smagorinsky model is relatively better compared to other three SGS models at both locations. It is to be noted that when a coarser grid is used, a thicker boundary layer is predicted near the surfaces compared to the simulations where a finer grid is used. An 
example of contour plots is given as Figure C.1. in Appendix C. As a result to maintain the flow continuity, higher boundary layer edge velocity is predicted for coarser grid.

Turbulent flows are highly diffusive in nature due to continuous three-dimensional fluctuations of the fluid throughout the computational domain. Turbulence intensity arises due to the nonlinear diffusive nature of fluid flow. It is an important flow parameter to quantify the nature of the turbulent flow.

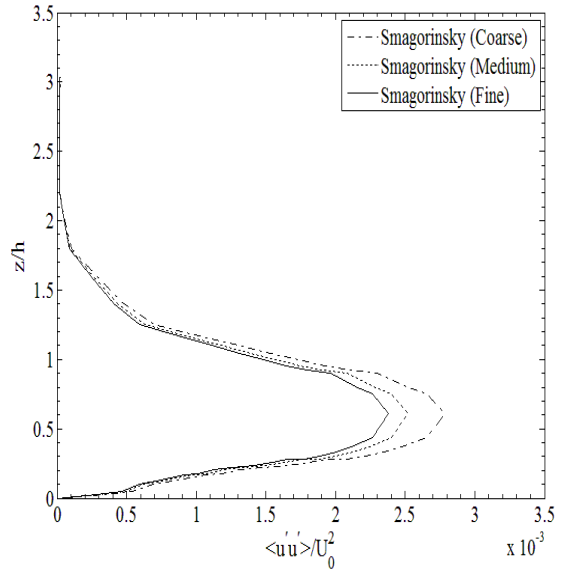

(a) Constant Smagorinsky

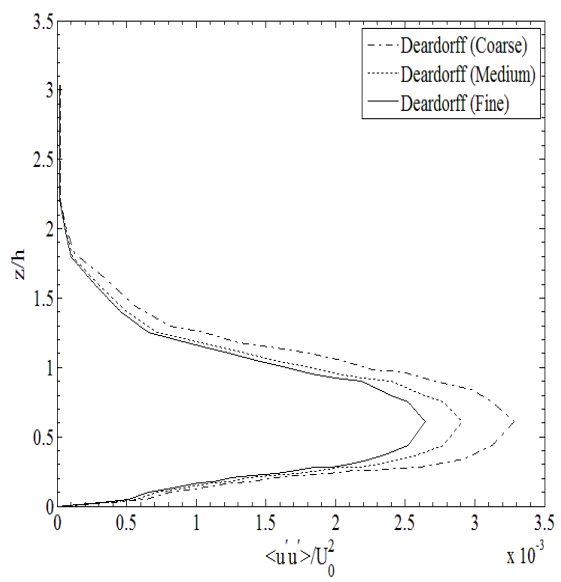

(c) Deardorff

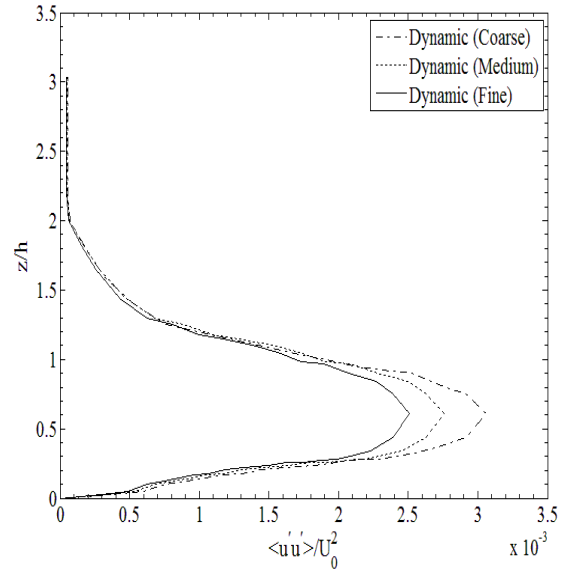

(b) Dynamic Smagorinsky

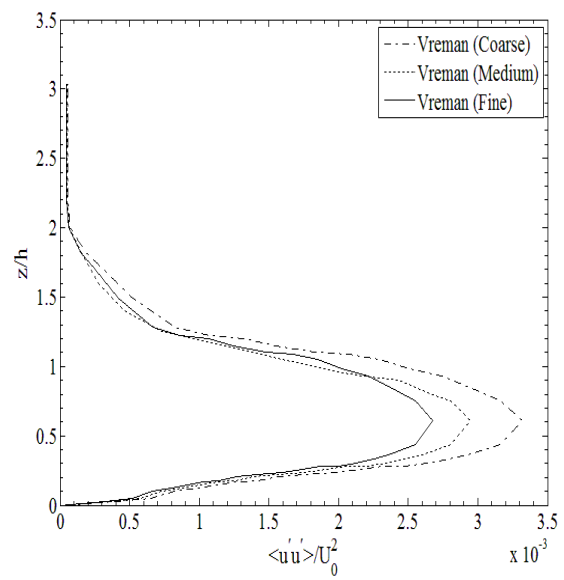

(d) Vreman

Figure 3.5. Turbulence intensities at test section $x / h=6$ for the SGS models

The rms profiles of the turbulence intensities or Reynolds stresses $\left(\overline{u^{\prime} u^{\prime}}\right)$ at different locations along the streamwise direction are normalized by the reference velocity $U_{0}^{2}$. Figures 3.4 and 3.5 present the turbulence intensities at two test locations $x / h=4$ and $x / h=6$ for the SGS models. 
Similar to the mean velocity profile, it appears from the figure that SGS models turbulence intensity profiles at selected test locations are unable to achieve grid convergence. Out of four models, it also appears the tendency of grid convergence of the standard Smagorinsky model is relatively better compared to the other three models which is consistent with figures 3.1 and 3.2. From these figures, it is observed that although the grids are sufficiently refined ( up to $n_{\min }^{+} \approx$ 1) but none of the SGS models is able to attain the numerical convergence.

\subsubsection{Quantitative comparison - mean velocity and turbulence intensity}

From the above graphical presentation of the grid sensitivity study of the mean velocity and turbulence intensity, it appears that none of the flow variables is able to attain grid convergence despite sufficient refinement of the grid resolution. Hence, it is important to evaluate the grid convergence tendency of the four models to identify the most promising one. In this section, the focus was given on estimation of grid convergence error of the mean velocity and the turbulence intensity to quantify their tendency towards the grid convergence.

The grid convergence method was proposed by Roache (1992) to estimate the grid convergence error which is based on the Richardson extrapolation. They point out that a grid convergence study requires a minimum of three grid solutions. The grid convergence index $(G C I)$ provides a measure of convergence for grid refinement studies. GCI (\%) can be expressed as follows

$$
G C I(\%)=F_{S} \frac{\epsilon_{r m s}}{r^{p}-1} \times 100
$$

Where, $p$ is the order of the numerical scheme and for second-order scheme $p=2, F_{s}$ is the factor of safety and the recommended value is $F_{s}=3$ (Roache 1992), $r$ represents the grid refinement ratio, can be calculated as $r=\left(N_{\text {fine }} / N_{\text {coarse }}\right)^{1 / 3}$, and $\epsilon_{r m s}$ is the $r m s$ value of the relative error which provide an initial measure of grid convergence for individual points $n$ as 


$$
\epsilon_{r m s}=\left(\frac{\sum_{i=1}^{n} \epsilon_{i}^{2}}{n}\right)^{1 / 2}
$$

where, the relative error $\left(\epsilon_{i}\right)$ at a certain point for a flow variable (say velocity $u_{i}$ ) can be calculated as the magnitude between the coarse and fine solutions

$$
\epsilon_{i}=\left|\frac{u_{i, \text { coarse }}-u_{i, \text { fine }}}{u_{i, \text { fine }}}\right|
$$

GCI has been calculated for three grid resolutions presented in Table 3.1. Results of this comparison in the form of grid convergence values (GCI) of the mean velocity and the turbulence intensity are reported in Tables 3.2 and 3.3. From the comparison, it is found that there is a reduction in $G C I$ values in the successive grid refinements $\left(G C I_{M / C}<G C I_{F / M}\right)$ for each of the two variables for the SGS models. The GCI of finer grid $\left(G C I_{F / M}\right)$ is relatively low compared to the coarser grid $\left(G C I_{M / C}\right)$ which indicates that the dependency of the numerical solutions of the simulations on the grid size have been reduced.

Table 3.2. Grid convergence measures (GCI) of the mean velocity profiles at stations $x / h=4$ and $x / h=6$ for flow over a backward facing step

\begin{tabular}{|c|c|c|c|c|c|}
\hline \multirow{2}{*}{ Grids } & Test & \multicolumn{4}{|c|}{ Grid convergence index (\%) } \\
\cline { 3 - 6 } & $\begin{array}{c}\text { sections } \\
(x / h)\end{array}$ & Smagorinsky & $\begin{array}{c}\text { Dynamics } \\
\text { Smagorinsky }\end{array}$ & Deardorff & Vreman \\
\hline Medium- Coarse (M/C) & 4 & 12.65 & 16.19 & 19.33 & 22.16 \\
\hline Fine- Medium (F/M) & 4 & 6.32 & 10.03 & 15.17 & 14.52 \\
\hline Medium-Coarse (M/C) & 6 & 13.12 & 15.94 & 16.87 & 19.57 \\
\hline Fine-Medium (F/M) & 6 & 6.45 & 10.31 & 12.68 & 14.11 \\
\hline
\end{tabular}

Table 3.3. Grid convergence measures (GCI) of the turbulence intensity at stations $x / h=4$ and $x / h=6$ for flow over a backward facing step

\begin{tabular}{|c|c|c|c|c|c|}
\hline \multirow{2}{*}{ Grids } & Test & \multicolumn{4}{|c|}{ Grid convergence index (\%) } \\
\cline { 3 - 6 } & $\begin{array}{c}\text { sections } \\
(x / h)\end{array}$ & Smagorinsky & $\begin{array}{c}\text { Dynamics } \\
\text { Smagorinsky }\end{array}$ & Deardorff & Vreman \\
\hline Medium-Coarse (M/C) & 4 & 15.18 & 22.67 & 27.06 & 31.02 \\
\hline Fine-Medium (F/M) & 4 & 7.58 & 14.04 & 21.24 & 20.33 \\
\hline Medium-Coarse (M/C) & 6 & 15.74 & 22.32 & 23.62 & 27.40 \\
\hline Fine-Medium (F/M) & 6 & 7.38 & 14.43 & 14.75 & 19.75 \\
\hline
\end{tabular}


From the comparison of $G C I$, it can be seen that the GCI value is almost halved for the standard Smagorinsky model between $\left(G C I_{M / C}\right)$ and $\left(G C I_{F / M}\right)$, whereas other three SGS models only reduced the error by roughly one-third. This quantitatively shows that among the four SGS models the grid convergence tendency of the standard Smagorinsky model is relatively high compared to the other three models. Roy (2003) recommended that GCI is should be $\leq 4 \%$ for both higher and lower order numerical schemes to confirm the quality of simulations. However further grid refinement may provide reduction in GCI leading to grid converged solutions (especially for the standard Smagorinsky model) which may fall in the DNS resolution range $\left(n_{\min }^{+} \approx 0.3\right)$.

\subsection{Results and discussions - predicted outcomes}

The quality and efficiency of the SGS models can be justified by comparing the best converged LES solution (based on the grid convergence study shown above) with experimental results of Jovic and Driver (1994). This is done quantitatively and graphically in this section.

\subsubsection{Statistical analysis of predicted outcomes}

The results using the fine mesh have been selected to determine the statistical relative error between the numerical solution and the experimental data of Jovic and Driver (1994). The method of Ierardi et al. (2003) has been followed. According to this approach, the relative error $(\xi)$ can be calculated as,

$$
\xi=\frac{(\mathcal{R}-\psi)}{\psi}
$$

where, $\mathcal{R}$ and $\psi$ are the reference and predicted values respectively. The mean relative error $\left(\xi_{m}\right)$ can be calculated by the absolute values of error in individual points $n$. Therefore, the overpredictions or under-predictions are of the same magnitude and the resultant error will not be zero, 


$$
\xi_{m}=\frac{\sum_{i=1}^{n}\left(\left|\frac{\mathcal{R}-\psi}{\psi}\right|\right)}{n}
$$

The relative errors are presented in Tables 3.4 and 3.5 for the mean velocity and turbulence intensity profiles. Relative errors for the SGS models considered are calculated by taking average of the relative mean errors at each of the two stations. From the Table 3.4, as can be seen, the relative error of the Smagorinsky model at fine resolution is almost two fold less than those of the dynamic Smagorinsky and the Deardorff models and almost three folds less than the Vreman model. Therefore, on the basis of mean relative error analysis, it can be said that standard Smagorinsky is the most efficient model (in terms of obtaining closest to experimental results with the same grid resolution) among the four SGS models for the considered test case. Table 3.5 results show a sharp increase of relative error in the turbulence intensities than the mean velocity components which indicate that the turbulence intensities are more sensitive to the SGS models. However, once again the standard Smagorinsky model is found to be the most efficient model, this time in relation to the prediction of turbulence intensities.

Table 3.4. Relative error analysis of the mean velocity profile (fine resolution) at stations $x / h=4$ and $x / h=6$ for flow over a backward facing step

\begin{tabular}{|c|c|c|c|c|}
\hline $\begin{array}{c}\text { Test Locations } \\
(x / h)\end{array}$ & Smagorinsky $(\%)$ & $\begin{array}{c}\text { Dynamics } \\
\text { Smagorinsky }(\%)\end{array}$ & Deardorff $(\%)$ & Vreman $(\%)$ \\
\hline 4 & 9.32 & 18.18 & 22.21 & 27.59 \\
\hline 6 & 12.56 & 19.45 & 23.58 & 28.93 \\
\hline Average & 10.94 & 18.81 & 22.90 & 28.26 \\
\hline
\end{tabular}

Table 3.5. Relative error analysis of the turbulence intensity (fine resolution) at stations $x / h=4$ and $x / h=6$ for flow over a backward facing step

\begin{tabular}{|c|c|c|c|c|}
\hline $\begin{array}{c}\text { Test Locations } \\
(x / h)\end{array}$ & Smagorinsky $(\%)$ & $\begin{array}{c}\text { Dynamics } \\
\text { Smagorinsky }(\%)\end{array}$ & Deardorff $(\%)$ & Vreman $(\%)$ \\
\hline 4 & 15.04 & 25.45 & 31.09 & 39.62 \\
\hline 6 & 17.62 & 28.33 & 33.01 & 36.50 \\
\hline Average & 16.33 & 26.89 & 32.05 & 38.06 \\
\hline
\end{tabular}




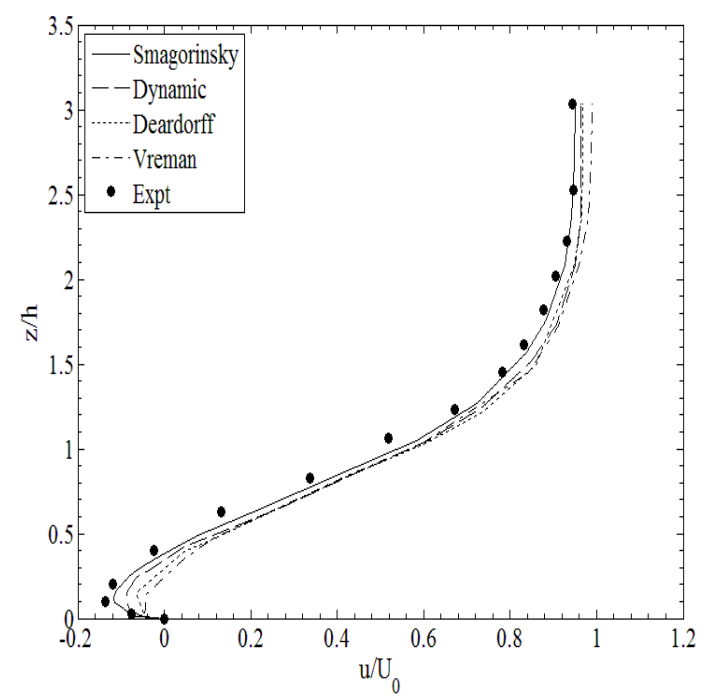

(a) at section $x / h=4$

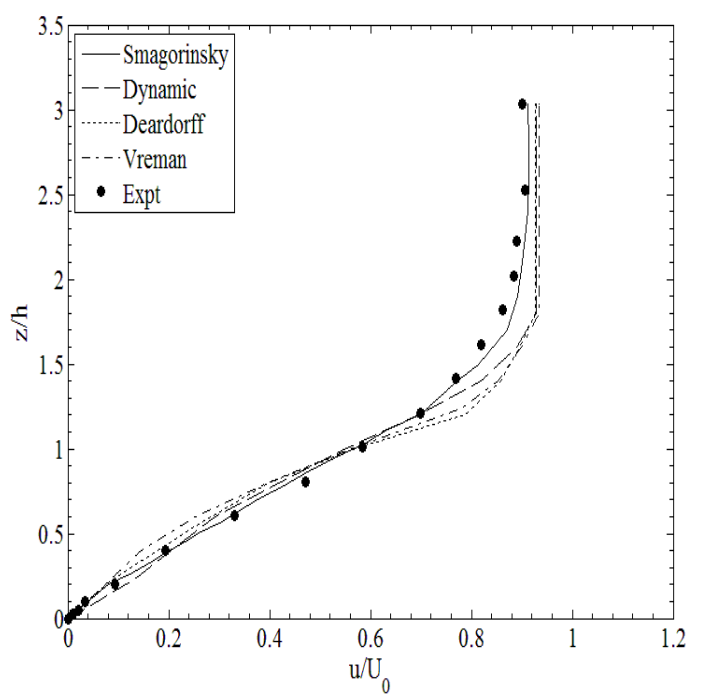

(b) at section $x / h=6$

Figure 3.6. Comparison of the mean velocity profile at test sections $x / h=4$ and $x / h=6$ for the SGS models

\subsubsection{Graphical Comparison}

\section{Mean velocity}

Figure 3.6 represents the results for the dimensionless mean streamwise velocity at test sections $x / h=4$ and $x / h=6$ at a fine grid resolution. In Figure 3.6 (a), at section $x / h=4$, it appears that a reverse flow takes place between the step height level and wall region. It seems that the standard Smagorinsky model captures the secondary vortices near the step relatively better than other models and shows relatively better agreement with the experimental data. At the same location, among the other three eddy models, the Deardorff and the dynamic Smagorinsky model display almost the same phenomena except near the step height, but the Vreman model demonstrates poor agreement with experimental values compared to the other two models in the region near the step height and away from the wall. At section $y / h=6$ (Figure $3.6(b)$ ), it is appears that the flow is attached to the wall. In that section the standard Smagorinsky model shows relatively better agreement with experimental data but other models exhibit moderate agreement along the 
vertical axis. From the comparison, as can be clearly seen, the most accurate model (for the given absolute spatial resolution) overall is the standard Smagorinsky model, the dynamic Smagorinsky model is the intermediate and the Deardorff and the Vreman model are the least well performing SGS models.

\section{Turbulence intensity}

Figure 3.7 represents the results comparison for turbulence intensities at selected test locations at fine grid resolutions. At test sections $x / h=4$ (Figure 3.7(a)) and $x / h=6$ (Figure 3.7(b)), it is found that similar to the mean velocity profiles the overall agreement of the turbulence intensity profiles of the standard Smagorinsky model with experimental data is relatively better compared to the other models. From the comparison, it can be concluded that the most accurate model (for the given absolute spatial resolution) overall is the standard Smagorinsky model, the dynamic Smagorinsky model is the intermediate and the Deardorff and the Vreman model are the least performing SGS models at both test locations for the turbulent intensities which is consistent with the mean velocity profiles in figure 3.6.

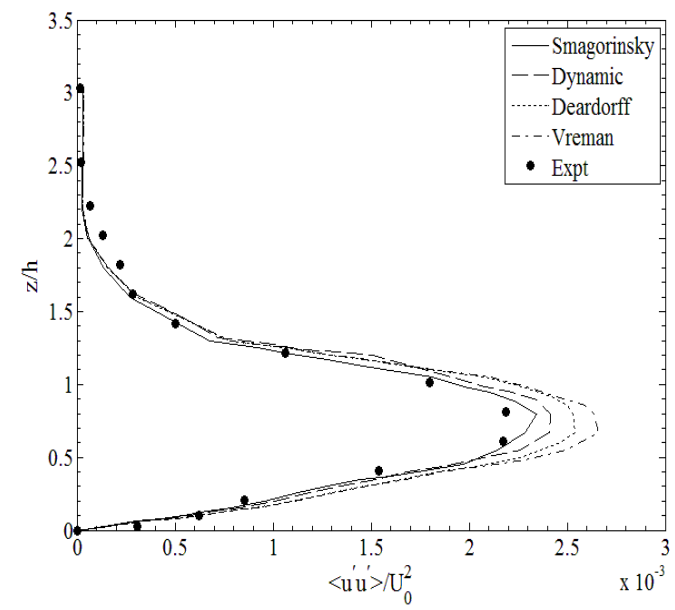

(a) at section $x / h=4$

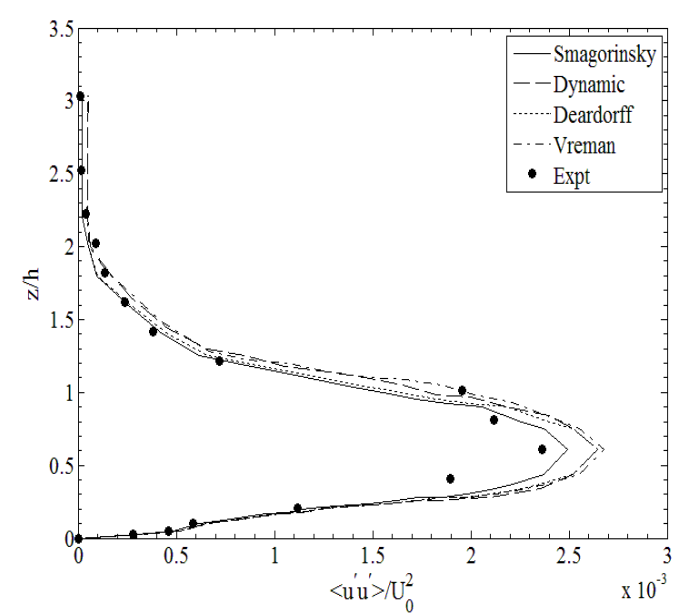

(b) at section $x / h=6$

Figure 3.7. Comparison of the turbulence intensity profile at test sections $x / h=4$ and $x / h=6$ for the SGS models 
In summary, from the grid convergence study and the statistical relative error analysis along with graphical comparison of the mean velocity and the turbulence intensity profiles with experimental data, it appears that the standard Smagorinsky model is the most promising SGS model in FDS out of four despite it is unable to achieve grid convergence. Akselvoll and Moin (1993) application of LES to a backward facing step with $R e_{h}=5100$ (based on the step height; $h$ ) shows that even with $n_{w a l l}^{+}=0.8$ resolution, they were unable to achieve a grid converged solution with implicitly filtered LES. It seems the results of this present study are consistent with the findings of Akselvoll and Moin (1993) and Toms (2015) which also exhibit the elusive nature of grid convergence in implicitly filtered LES. However another simulation is conducted with the most promising standard Smagorinsky model when the grid resolution is further refined to $h / \delta z=20$. This is to assess the adequacy of the second-order scheme.

\subsection{Further simulation with standard Smagorinsky model}

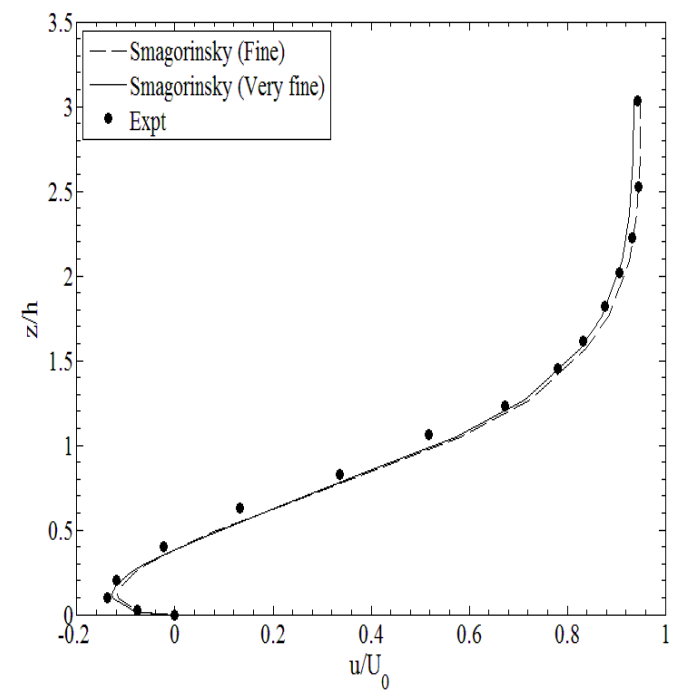

(a) at section $x / h=4$

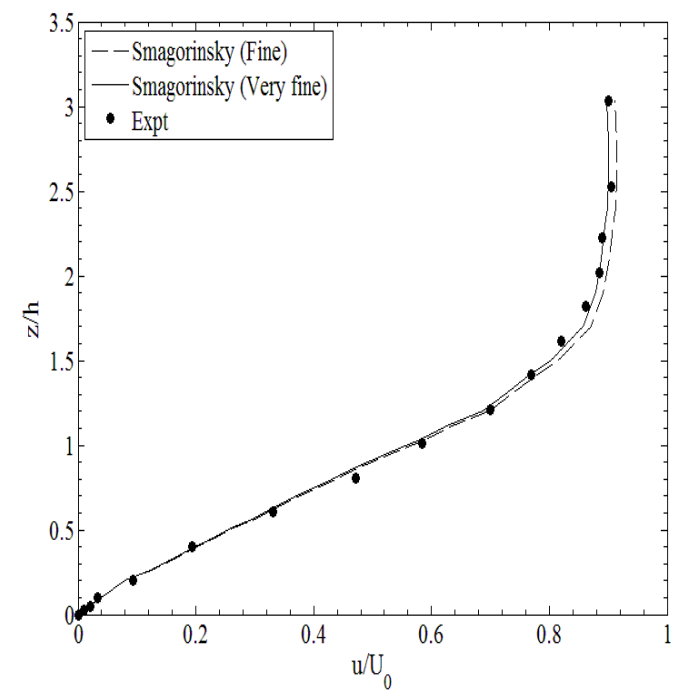

(b) at section $x / h=6$

Figure 3.8. Comparison of the mean velocity profile at test sections $x / h=4$ and $x / h=6$ for the standard Smagorinsky model for $h / \delta z=20$ 


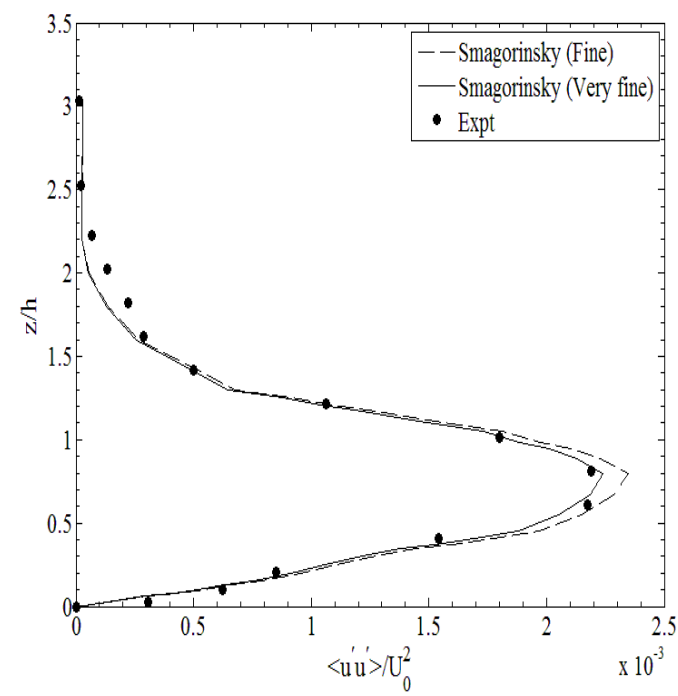

(a) at section $x / h=4$

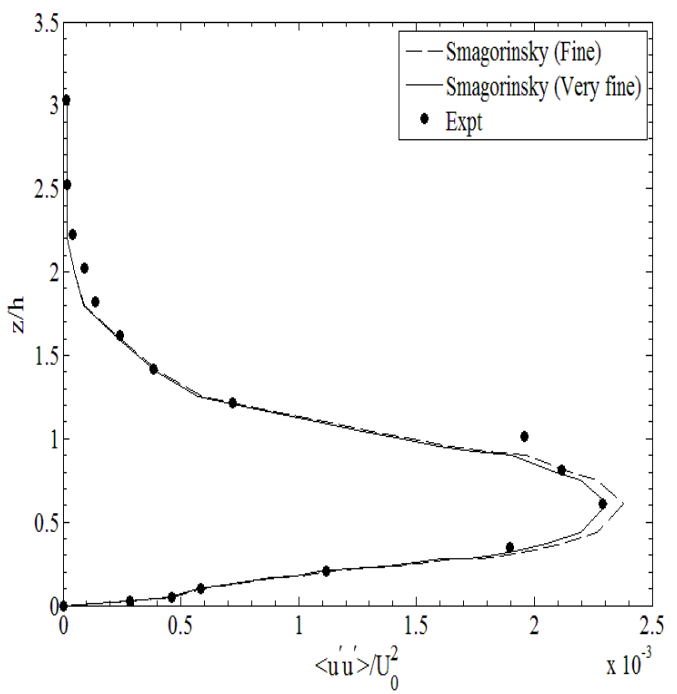

(b) at section $x / h=6$

Figure 3.9. Comparison of the turbulence intensity profile at test sections $x / h=4$ and $x / h=6$ for the standard Smagorinsky model for $h / \delta z=20$

To assess the adequacy of second-order scheme, the grid is further refined as $h / \delta z=20$ (very fine) where the grid resolution is $240 \times 600 \times 400$ and the nominal wall unit is measured as $n_{\text {min }}^{+} \approx 0.46$. Results of the mean velocity and the turbulence intensity of very fine grid (VF) are presented in the Figures 3.8 and 3.9 along with fine grid $(F)$ to show VF's tendency of grid converge and also compared with the experimental data. From the figures, it can be said that for both mean velocity and turbulence intensity, very fine grid shows relatively better agreement with experimental data compared to fine grid. In the similar way, grid convergence index and relative errors of the mean velocity and the turbulence intensity have been calculated to assess the grid convergence tendency and the relative accuracy of the model with VF grid that are presented in the Tables 3.6 and 3.7 respectively.

As mentioned earlier Roy (2003) recommended a GCI value of $\leq 4 \%$ to confirm the quality of simulations. From the Table 3.6, it appears that GCI $(\%)$ of the flow variables are around this range, but still some grid sensitivity remains. 
Table 3.6. GCI of the mean velocity and the turbulence intensity profiles of standard

Smagorinsky model at stations $x / h=4$ and $x / h=6$ with very fine grid $(h / \delta z=20)$

\begin{tabular}{|c|c|c|c|}
\hline \multirow{2}{*}{ Grid } & Test section & \multicolumn{2}{|c|}{ Grid convergence index (\%) } \\
\cline { 3 - 4 } & $(x / h)$ & Mean velocity & Turbulence intensity \\
\hline \multirow{2}{*}{ Very fine-Fine $(\mathrm{VF} / \mathrm{F})$} & 4 & 3.27 & 4.18 \\
\cline { 3 - 4 } & 6 & 3.43 & 4.07 \\
\hline
\end{tabular}

Table 3.7. Relative errors of the mean velocity and the turbulence intensity profiles of standard Smagorinsky model at stations $x / h=4$ and $x / h=6$ with very fine grid $(h / \delta z=20)$

\begin{tabular}{|c|c|c|}
\hline \multirow{2}{*}{$\begin{array}{c}\text { Test section } \\
(x / h)\end{array}$} & \multicolumn{2}{|c|}{ Relative errors (\%) } \\
\cline { 2 - 3 } & Mean velocity & Turbulence intensity \\
\hline 4 & 4.38 & 6.58 \\
\hline 6 & 5.73 & 7.12 \\
\hline Average & 5.05 & 6.85 \\
\hline
\end{tabular}

Accuracy of the obtained solution of the mean velocity and the turbulence intensity is presented in the Table 3.7. Table 3.7 shows that average relative errors of the mean velocity and the turbulence intensity at two stations are approximately 5\% and 7\%. According to Roache (1998) and Roy (2003), for the numerical validation of turbulent flow cases errors are expected to be within $5 \%$. Therefore it can be observed that obtained results of mean flow from very fine grid (VF) simulation are almost within acceptable range. The turbulence intensity failed to satisfy this condition. However from the trend, it can be expected that further refinement of grid may provide more accurate results but it will be similar or higher than that of grid resolution considered in DNS (Le, Moin and Kim 1997) in terms of nominal $n_{\min }^{+}$. This shows the elusiveness of grid convergence. This particular simulation using standard Smagorinsky model with very fine grid reduces the relative errors of both flow variables within the acceptable range and shows very good agreement with the experimental data which demonstrates the adequacy of the second-order numerical scheme. In the process, this study also shows the elusiveness of the grid convergence of implicitly filtered LES. 


\subsection{Conclusions}

For similar inlet boundary conditions fluid flow over a backward facing step is simulated using four different SGS models in FDS to identify the most promising SGS model and to assess the adequacy of a second-order numerical scheme. This chapter shows that grid resolutions have a stronger influence on the performance of the SGS models considered in implicitly filtered LES. According to the results of the GCI analysis, the standard Smagorinsky eddy viscosity model is found to be the most converging model compared to other three LES models considered. Based on this, an extra simulation with finer resolution (nominal $n^{+} \approx 0.46$ ) with standard Smagorinsky model is conducted. Analysis of the results of the further simulation with standard Smagorinsky model in terms of relative error and tendency of GCI justifies the adequacy of second-order numerical scheme. This study also demonstrates the elusiveness of grid convergence of implicitly filtered LES. A particular finding of this study is the justification of the modification of LES's standard Smagorinsky eddy model to an explicitly filtered LES scheme as outlined in Chapter 2. 


\section{Chapter 4}

\section{High Reynolds number turbulent flow}

The literature suggests that highly turbulent flows exhibit an inertial subrange in the energy spectrum (Mansour et al. 1993, Pope 2004). However, it is important to ensure that the explicit scheme can effectively capture this range. It is also important that filter widths selected on the basis of BLT in explicit schemes fulfil the criteria of the principle of LES for obtaining appropriate numerical solutions. The effectiveness of explicit filtering in high Reynolds number turbulent flow will be demonstrated in this chapter. A well-defined problem is studied, namely turbulent flow over a backward facing step, which has been studied in Chapter 3 with implicit LES schemes, to provide a comparator for investigating the effectiveness of the explicit scheme.

\subsection{Studied numerical configuration}

In this study, turbulent flow over a backward facing step is considered to study high Reynolds number flows. The Reynolds number based on Taylor's scale $\left(R e_{\lambda}\right)$ is calculated to be approximately 115 . The computational domain and the boundary conditions of the numerical simulation of the backward facing step were set up as done in Chapter 3 and the rationale is provided there. In this chapter, the numerical results of turbulent flow over a backward facing step obtained using an explicit LES scheme are compared with the existing experimental results.

\section{Filter to grid spacing ratio (FGR)}

In this study, for the high Reynolds number flow over a backward facing step, the filter to grid spacing ratios (FGR) have been taken as 2, 4, 8 and 16. In Table 4.1, it is shown that a total of eight simulations need to be performed for two filter widths $(\Delta)$ that are selected as $10 \%$ and 
$20 \%$ of the boundary layer thickness (BLT). The intention is to select $5 \%$ of BLT as the filter width for further simulations if the selected two do not provide results consistent with the LES principles discussed in Chapter 2.

The explicit filter width is directly linked to the BLT, the value of which must be estimated. The BLT is conventionally defined as the normal distance from the wall at which the local streamwise flow velocity $(u)$ reaches $99 \%$ that of the free stream velocity $\left(U_{e}\right)$. In the absence of experimental or DNS data, the BLT can be estimated from analytical expressions. In the absence of similar expressions for flow over a backward facing step this expression is used and a characteristic point is selected a distance $3 h$ upstream of the step which corresponds to the experimental reference point. The analytical expression for a flat plate boundary layer given by Schlichting (1979)

$$
\frac{\delta}{x}=\frac{0.382}{R e_{x}^{1 / 5}}
$$

where $x$ is the streamwise distance from the start of the boundary layer and $R e_{x}=U_{0} x / v$. It is recognized that the BLT is a function of the streamwise coordinate, however for the simplicity of the scheme, the BLT at a single reference point is considered in this study. The characteristic BLT is analytically estimated as $\delta=2.1 \times 10^{-2} \mathrm{~m}$ which is close to the experimentally observed value of $\delta=2.4 \times 10^{-2} \mathrm{~m}$ by Jovic and Driver (1994).

Table 4.1. Selected filter sizes $\Delta_{1}$ and $\Delta_{2}$ for a turbulent flow at high Reynolds numbers

\begin{tabular}{|c|c|c|c|c|}
\hline \multicolumn{2}{|c|}{ Case 1} & \multicolumn{2}{|c|}{ Case 2 } & Filter/grid ratio \\
\hline \multicolumn{2}{|c|}{ Fixed Filter width $\Delta_{1}=0.2 \delta$} & \multicolumn{2}{|c|}{ Fixed Filter width $\Delta_{2}=0.1 \delta$} & $\Delta / \delta \mathrm{x}$ \\
\hline Case 11 & $\delta \mathrm{x}=0.5 \Delta_{1}$ & Case 21 & $\delta \mathrm{x}=0.5 \Delta_{2}$ & 2 \\
\hline Case 12 & $\delta \mathrm{x}=0.25 \Delta_{1}$ & Case 22 & $\delta \mathrm{x}=0.25 \Delta_{2}$ & 4 \\
\hline Case 13 & $\delta \mathrm{x}=0.125 \Delta_{1}$ & Case 23 & $\delta \mathrm{x}=0.125 \Delta_{2}$ & 8 \\
\hline Case 14 & $\delta \mathrm{x}=0.0625 \Delta_{1}$ & Case 24 & $\delta \mathrm{x}=0.0625 \Delta_{2}$ & 16 \\
\hline
\end{tabular}


The rationale behind the selection of the two filter widths as a fraction of the BLT was previously discussed in section 2.3. For each filter the base case FGR is 2 because this ratio has been commonly adopted (Gullbrand 2002, Gullbrand and Chow 2003, Radhakrishnan and Bellan 2012, 2013 and 2015), and it provides a convenient starting point for our investigations. We subsequently refine the FGR to 4, 8 and 16 as we seek converged solutions.

\section{Grid resolutions of flow over a backward facing step}

For the high Reynolds number test case described in the previous section, a comprehensive set of eight explicitly filtered LES are conducted. This involves two different filter widths and four different FGR. These are summarised in Table 4.1. Table 4.2 presents the grid resolutions implemented in the numerical experiments on high Reynolds number flows. Grids presented in the Table 4.2 are different from the grids used in the Chapter 3 due to the grid selection procedure. In Chapter 3 grids are based on step height to grid spacing ratio $(h / \delta z)$ whereas here grids are seleted based on a certain percentage of BLT.

Table 4.2. Grid resolutions for flow over a backward facing step at a high Reynolds number,

$$
R e_{\lambda}=115
$$

\begin{tabular}{|c|c|c|}
\hline \multicolumn{3}{|c|}{$\begin{array}{c}\text { High Reynolds number turbulent flow over a backward facing step } \\
\text { (Estimated Reynolds number, } R e_{\lambda}=115 \text { ) }\end{array}$} \\
\hline Test cases & $N_{x} \times N_{y} \times N_{z}$ & $n_{\text {Wall }}^{+}$ \\
\hline Case 11 & $60 \times 20 \times 40$ & $\leq 4.23$ \\
\hline Case 12 & $120 \times 40 \times 80$ & $\leq 2.49$ \\
\hline Case 13 & $240 \times 80 \times 160$ & $\leq 1.13$ \\
\hline Case 14 & $480 \times 160 \times 320$ & $\leq 0.93$ \\
\hline Case 21 & $120 \times 40 \times 80$ & $\leq 2.32$ \\
\hline Case 22 & $240 \times 80 \times 160$ & $\leq 1.04$ \\
\hline Case 23 & $480 \times 160 \times 320$ & $\leq 0.79$ \\
\hline Case 24 & $960 \times 320 \times 640$ & $\leq 0.43$ \\
\hline
\end{tabular}

$N_{x}, N_{y}$ and $N_{z}$ represents the number of grids (or meshes) along the spanwise $\left(L_{x}\right)$, streamwise $\left(L_{y}\right)$ and wall normal $\left(L_{z}\right)$ length directions. The resolutions are given in the Table is in the downstream of the step of the test case. A rectangular grid was used in all simulations. The 
dimensionless wall units $\left(n^{+}\right)$are also presented in Table 4.2. It can be noted that due to the non-stretched grid, Case 23 and Case 24 have almost 3 and 20 times more grid cells than Le, Moin and Kim (1997).

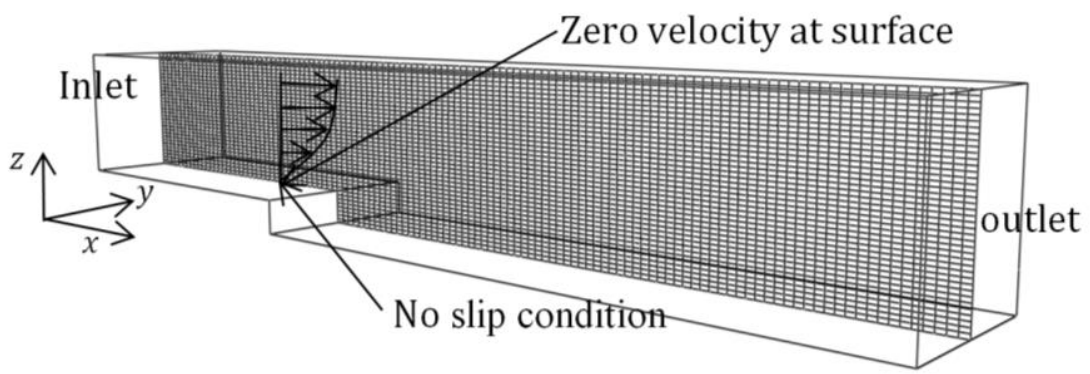

Figure 4.1. Schematic diagram of grid used in Case 12 for a backward facing step In their DNS study grid resolution was $786 \times 64 \times 192$ and non-uniform grid distribution is used along the vertical direction. They used fine grid near the bottom wall and coarse grid away from the wall and the wall unit ranging from $n_{\min }^{+} \approx 0.1$ to $n_{\max }^{+} \approx 31$. However, we have used uniform grid of equal sizes in all directions. Figure 4.1 presents the schematic of the computational domain and grid resolutions of Case 12 (presented in Table 4.2) to show the grid arrangement inside the test configuration during numerical simulation.

\subsection{Results and discussions}

The explicitly filtered LES solution is analysed in two ways. Firstly the energy spectra are examined to determine the explicit filter settings and grid resolutions required to achieve grid independent LES that captures the large scale energy containing range and the correct scaling in the inertial subrange. Unlike GCI in Chapter 3, the energy spectrum analysis is used for its robustness. Gullbrand (2002) adopted the similar techniques. Secondly the quality of the simulations is analysed by their ability to capture the prominent features of the flow and through a statistical comparison of the LES predictions against available experimental data for mean velocity and turbulent intensity. 


\subsubsection{Energy spectra analysis}

Figure 4.2 shows the grid convergence of energy spectra for the two sets of cases with different fixed filter widths: Case 1 with $\Delta_{1}=0.2 \delta$ and Case 2 with $\Delta_{2}=0.1 \delta$. The inertial subrange is identified in the figures by comparison to Kolmogorov's universal scaling line with slope of $-5 / 3$. The probable demarcation of the energy-containing and inertial ranges is also indicated by the vertical dotted line. Results obtained from the four values of FGR presented in Table 4.1 are compared. It can be seen that the energy spectra obtained in Case 13 with FGR $=8$ and Case 14 with FGR $=16$ are closely matched. Hence, it is considered that for a fixed filter $\Delta_{1}$ a gridconverged solution is obtained in Case 13. Similarly, convergence for the cases with a filter width $\Delta_{2}$ occurs when the FGR is increased to 8 (Case 23). It seems that the grid converged spectra for both cases with filter widths at $10 \%$ and $20 \%$ of the characteristic BLT satisfy the LES principles in that the energy containing range is captured and the filter widths are within the inertial subrange. For this high Reynolds number flow the grid converged energy spectra indeed conform to the $-5 / 3$ relationship as expected.

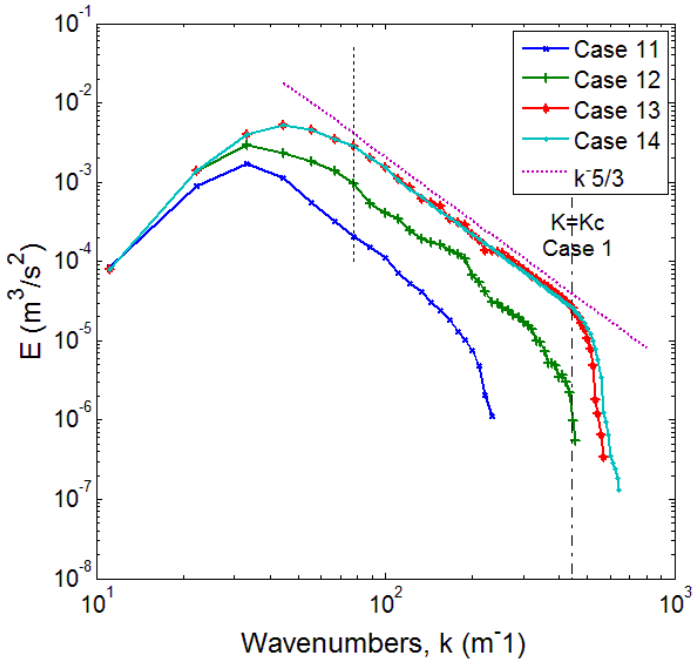

(a) Case $1\left(\Delta_{1}=0.2 \delta\right)$

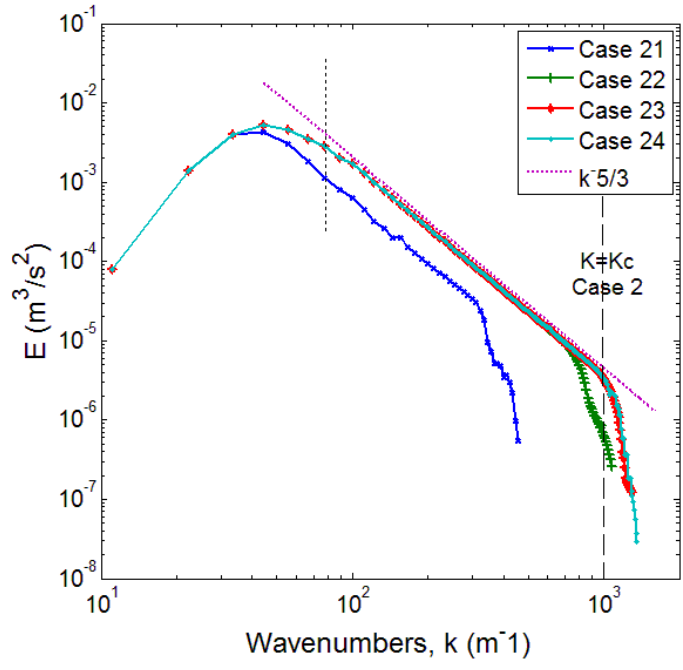

(b) Case $2\left(\Delta_{2}=0.1 \delta\right)$

Figure 4.2. Grid-convergence of three-dimensional energy spectra for the high Reynolds number flow with two different fixed filter widths. 
This apparent need for FGR $=8$ is at odds with some of the literature. As discussed earlier Gullbrand and Chow (2003) found that FGR $=4$ was sufficient in their second-order scheme. However, we see in Figure 4.2 that although Case 12 with FGR $=4$ is a long way from the gridconverged spectra, Case 22 also with FGR $=4$ is actually quite close to being grid-converged although the effective cut-off wavenumber (corresponding to the point where the spectrum tail shows a sudden decrease) is slightly less than in Case 23 and Case 24. This more rapid convergence with increasing FGR for the finer filter width cases suggests that the required minimum FGR is filter width dependent, and that smaller explicit filter widths may not require as intensive a grid resolution as do wider filter width simulations. It is also interesting to compare the nominal cut-off wave numbers corresponding to the filter given by $\kappa_{c}=\pi / \Delta$, which are indicated by vertical dashed-dotted lines in Figure 4.2 with the effective cut-off wave numbers. On the coarsest grid (Case 11$)$, it is found that the first grid point $\left(n^{+}\right)$at selected test sections are located 4 plus-units (see Table 4.2) above the wall. It appears on such a grid it is not possible to obtain reasonable results which is evident from the Figure 4.2(a). We see for both cases that when FGR $=4$ (Case 12 and Case 22) the effective cut-off is quite close to the nominal cut-off $\left(\kappa_{c}=\pi / \Delta\right)$ but at the same time some of the large energy containing eddies are also being filtered out as indicated by a failure of the Case 12 spectra to reach the peak low wave number energy. On the other hand when the FGR is increased, although the peak energy levels are being reached, the effective cut-off wave numbers are slightly above the nominal cut-off. This larger than expected cut-off is an artefact of the filtering method and the need to increase FGR to a sufficiently high level to capture the energy containing range at the expense of over-resolving the inertial subrange represents an inefficiency in the approach; albeit quite a small and tolerable inefficiency in these cases shown here. It is well known that spatial filtering operations are not 
"sharp" and that some of the turbulent motions captured on the grid remain unfiltered due to the nature of the filtering (here that is a weighting of grid values). Spectral filters, although potentially difficult to implement, are more efficient as they may achieve a desired sharp cut-off (Ghosal 1996).

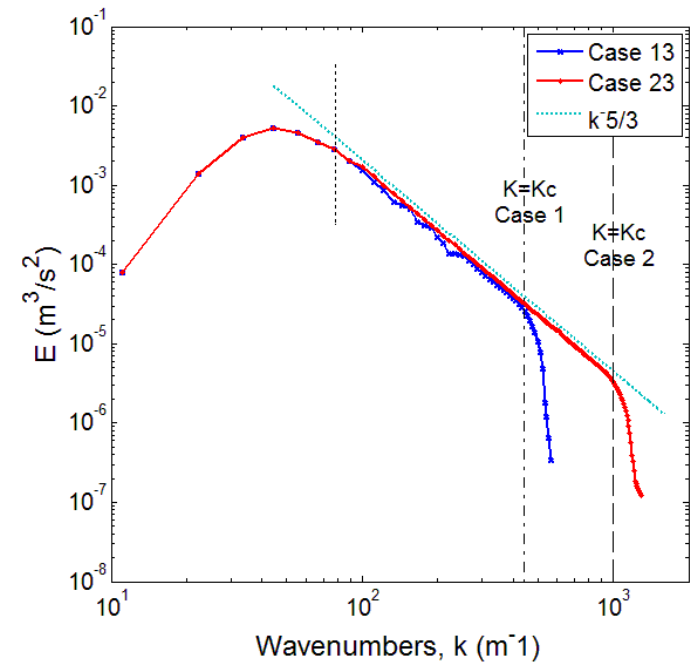

(a) Grid converged energy spectra of Case 1 $\left(\Delta_{1}=0.2 \delta\right)$ and Case $2\left(\Delta_{2}=0.1 \delta\right)$

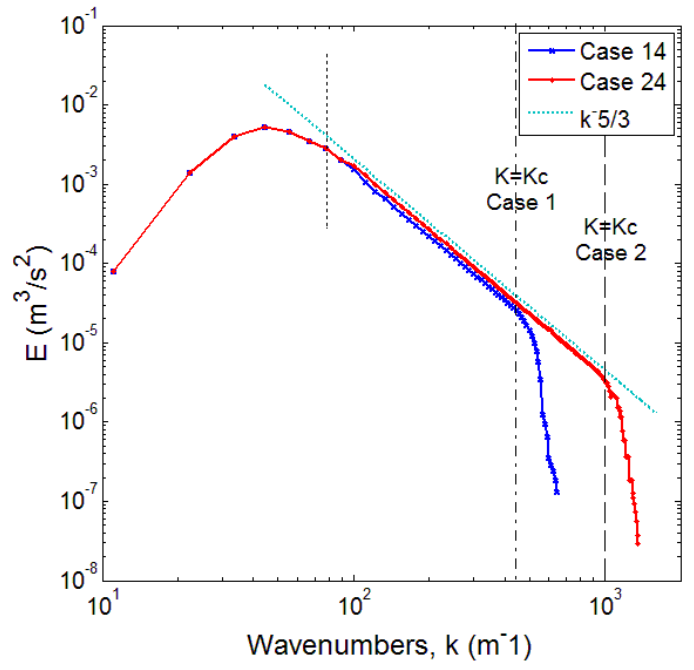

(b) Energy spectra obtained using the finest grids of Case $1\left(\Delta_{1}=0.2 \delta\right)$ and Case $2\left(\Delta_{2}=0.1 \delta\right)$

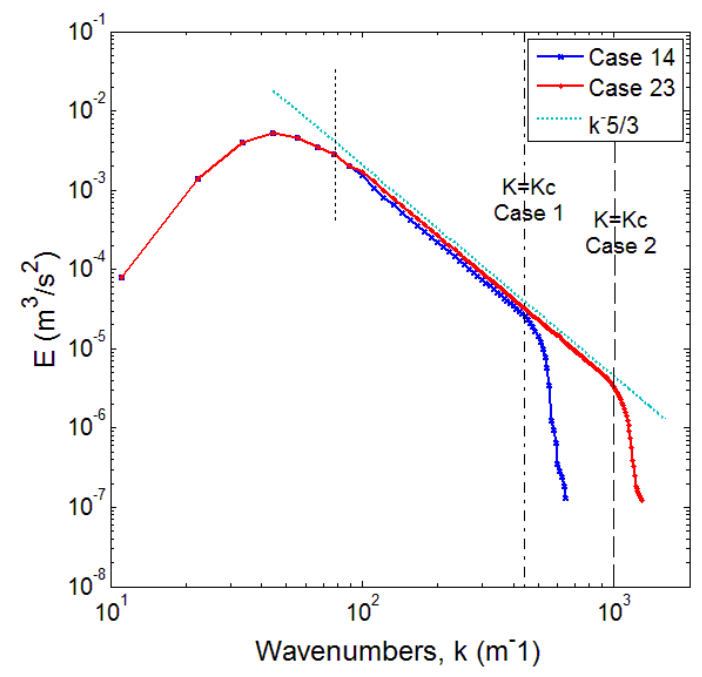

(c) Equal grid resolutions for Case $1\left(\Delta_{1}=0.2 \delta\right)$ and

Case $2\left(\Delta_{2}=0.1 \delta\right)$

Figure 4.3. Energy spectra analysis for cases in which the grid size is maintained constant and the filter width is varied. Case $1\left(\Delta_{1}=0.2 \delta\right)$ and Case $2\left(\Delta_{2}=0.1 \delta\right)$ 
Grid-converged spectra profiles of both filter width cases are compared in different combinations such as; (a) relatively coarse grids (Case 13 \& Case 23), (b) finest grids (Case 14 \& Case 24) and (c) same grid size but different filter widths (Case 14 \& Case 23). A comparison of gridconverged solutions of energy spectra taken from Figure 4.2 for filter width $\Delta_{1}$ and $\Delta_{2}$ is presented in Figure 4.3. From the figure, it is found that converged solutions of both fixed filter widths are in very good agreement with the plotted $-5 / 3$ slope. Moreover, taken filter widths are lying in the inertial subrange as expressed by dashed vertical lines and captured all the energy containing eddies are captured properly. It appears from Figure 4.3(a) that Case 23 is more likely to provide more accurate solution compared to Case 13, but Case 13 is deemed acceptable from the LES principle as well as the requirement for numerical convergence. The comparison for finest grid resolutions, Case 14 and Case 24, which are also grid-converged solutions of energy spectra for fixed filter widths $\Delta_{1}$ and $\Delta_{2}$ are presented in Figure 4.3(b). It is seen that the spectra profiles follow the same pattern as in the earlier comparison. However, from the comparison it appears that a comparatively fine grid captures higher wavenumbers compared to relatively coarse grid while the filter width is the same and the solutions with both grids appear to have negligible numerical error.

The application of LES is often motivated by a desire to predict turbulent flows at as low a computational cost as possible; and certainly at a cost significantly below that of DNS. To this end it is interesting to compare results for two different filter widths but with the same grid resolution and hence approximately the same computational cost. Figure 4.3(c) shows the energy spectra for Cases 14 and 23. Both simulations are grid-converged, both cost about the same to compute and both conform to the principles of LES. The latter case has a higher effective cut-off wavenumber due to its having a smaller filter width. As shown in Figure 4.2, Case 13 produces the same spectral result as does Case 14 but at half the grid resolution in each coordinate 
direction. When combined with a higher stable time step, Case 13 could cost up to 16 times less to compute than either Case 14 or Case 23. Producing energy spectra which conform to LES principles is but one criterion for judging LES quality. In Section 4.2.3.2 an error analysis relative to experimental data is conducted and further comment is made on both computational cost and the most appropriate filter width. Overall, the figure with all possible the grid converged solution combinations of Case $1\left(\Delta_{1}=0.2 \delta\right)$ and Case $2\left(\Delta_{2}=0.1 \delta\right)$ filter widths shows that all combinations satisfy the LES principle by capturing the energy containing range properly. Therefore no simulation is conducted taking $5 \%$ of BLT as the filter width.

\subsubsection{The congruence of energy spectra with flow variables}

Statistical quantities for this flow were collected by time averaging. In this flow case, it appears that the flow achieved statistically steady state at approximately $800 t_{E}$ with $5 \times 10^{-3} S$ recirculation (eddy turnover) time near the step height $(h)$. After an initial run of $800 t_{E}$, the flow velocity is averaged over the time for another $2000 t_{E}$. The average velocity data was obtained at 0.01s interval (that means nearly two eddy turnover time) and statistical averge of those 1000 data points was taken which is considered adequate. The time averaged velocity field corresponding to approximately 270 characteristic domain flow throughs. The effect of the filter width and grid size on energy spectra has been analysed in detail. This prompts one to investigate whether or not seemingly accurate simulations of the energy spectrum are reflected in the accuracy of two dimensionless flow variables, namely mean velocity and turbulence intensity. Both of the variables are important for describing the nature of the flow qualitatively and quantitatively.

\section{Mean velocity}

Figure 4.4 presents the mean velocity, obtained from Cases 11-14, normalised by the reference velocity for fixed filter width Case $1\left(\Delta_{1}=0.2 \delta\right)$ at four different streamwise test locations. The overall reference velocity is maintained within $1 \%$ of the ideally desired value $(7.77 \mathrm{~m} / \mathrm{s})$ at a 
distance of $3 h$ upstream of the step. From Figure 4.4, it appears that mean velocity profiles at different test locations in Case $13\left(\delta \mathrm{x}=0.125 \Delta_{1}\right.$ and FGR 8$)$ and Case $14\left(\delta \mathrm{x}=0.0625 \Delta_{1}\right.$ and FGR 16) are closely matched to each other which is consistent with our findings on the energy spectra shown in Figure 4.2(a). In both cases, $n_{\min }^{+}$is approximately 1 . Therefore, it can be said that mean velocity profiles are numerically converged at grid resolutions Case 13 . It can be seen in Figure 4.2(a) that for Case 11 and Case 12 some of the large energy containing eddies are being filtered out as indicated by a failure the spectra to reach the peak low wave number energy in these cases. This has a profound effect on the flow development and as a result the local Reynolds number is lower compared to those of Case 13 and Case 14.

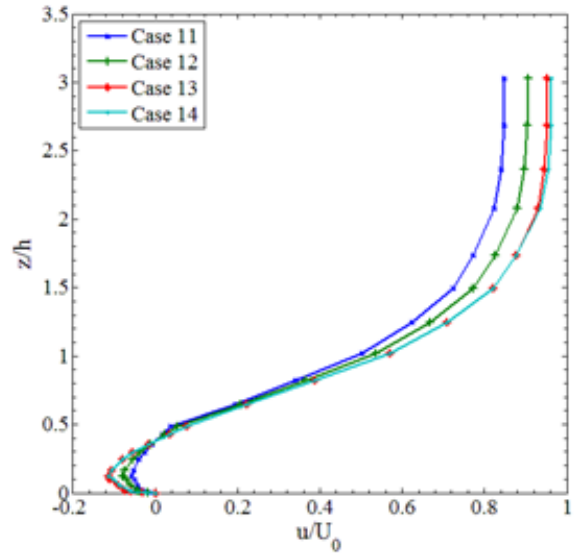

(a) At test section $x / h=4$

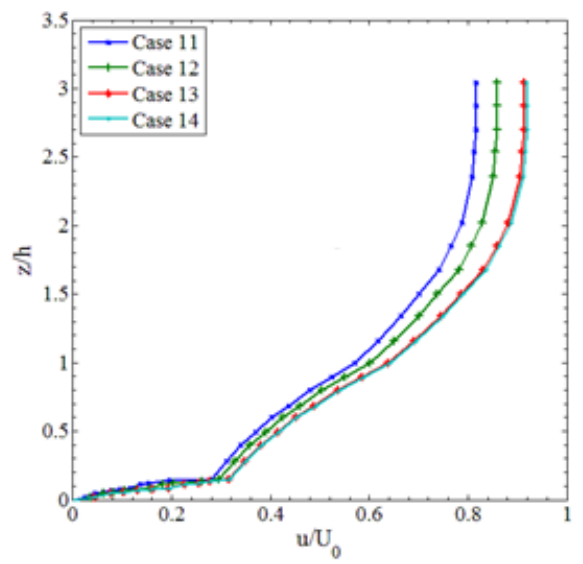

(c) At test section $x / h=10$

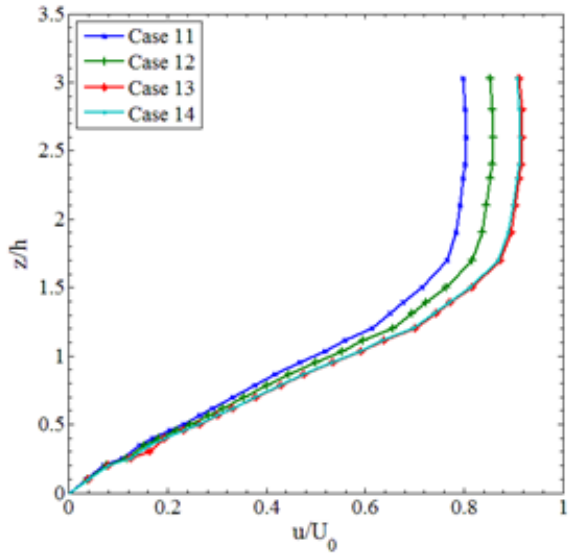

b) At test section $x / h=6$

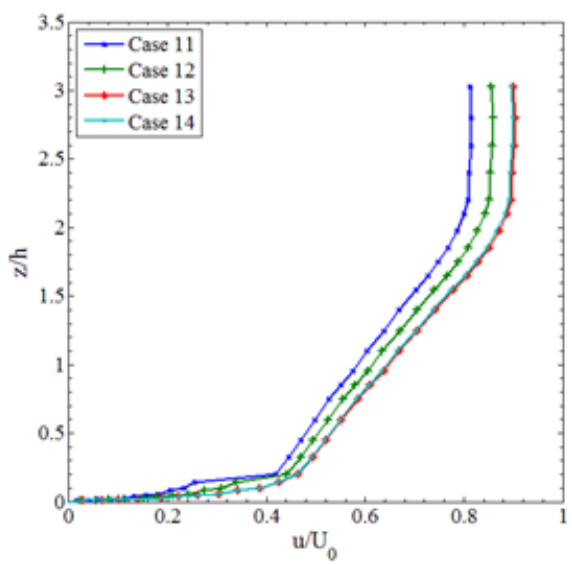

(d) At test section $x / h=19$

Figure 4.4. Mean velocity at four test sections for fixed filter Case $1\left(\Delta_{1}=0.2 \delta\right)$ 
Figure 4.5 shows normalized mean velocity profiles, obtained from Cases 21-24 (where $\Delta_{2}=$ $0.1 \delta)$ at four streamwise test locations. It can be observed that Case $23\left(\delta \mathrm{x}=0.125 \Delta_{2}\right.$ and FGR 8$)$, and Case $24\left(\delta \mathrm{x}=0.0625 \Delta_{2}\right.$ and FGR 16$)$ completely collapse onto each other and furthermore that the solutions for Case 22 are also very close to the solutions for Cases 23 and 24. This reflects Figure 4.2 (b) where these three cases are consistent with the LES principle. Furthermore, the nominal $n_{\text {min }}^{+}$value for these cases are 1 or below.

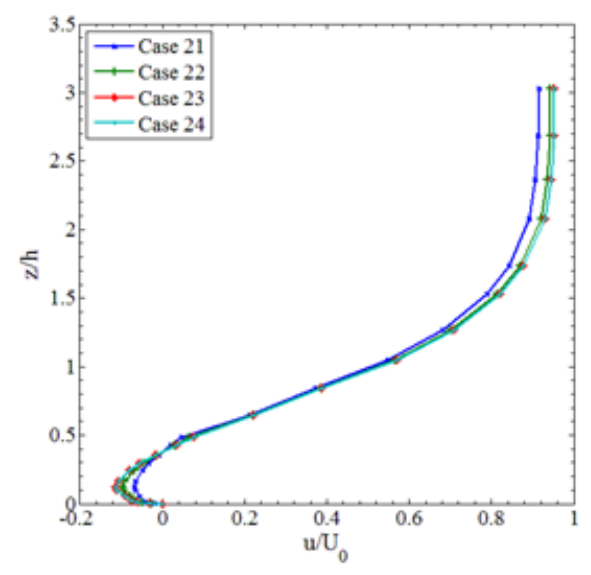

(a) At test section $x / h=4$

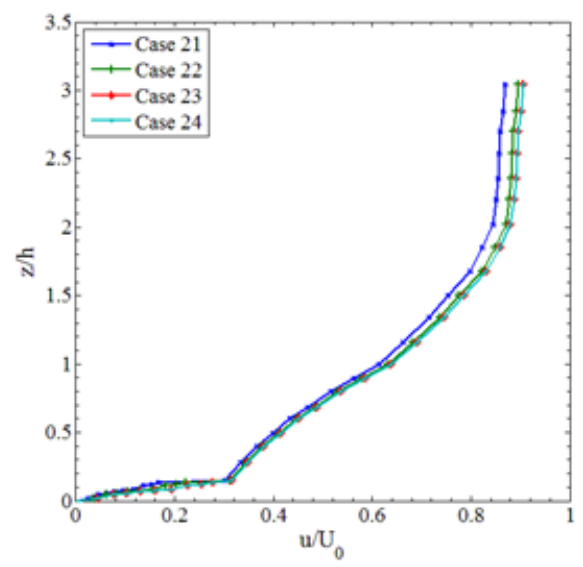

(c) At test section $x / h=10$

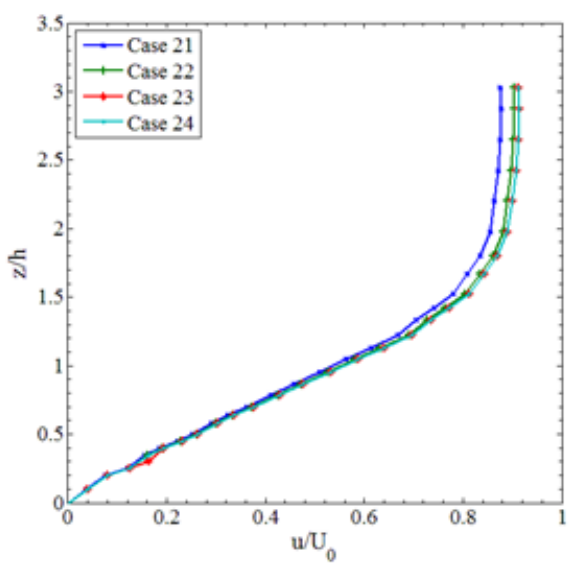

(b) At test section $x / h=6$

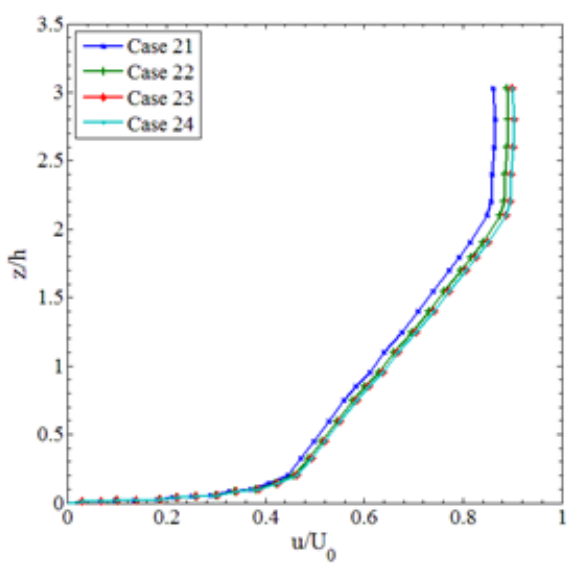

(d) At test section $x / h=19$

Figure 4.5. Mean velocity profiles at four test sections for fixed filter Case $2\left(\Delta_{2}=0.1 \delta\right)$

\section{Turbulence intensity}

Similar to the mean velocity profile, reflection of energy spectra on turbulence intensities has been presented in Figure 4.6 and 4.7 for filter width Case $1\left(\Delta_{1}=0.2 \delta\right)$ and Case $2\left(\Delta_{2}=0.1 \delta\right)$ at 
the same test locations of the backward facing step flow. From Figure 4.6, for Case $1\left(\Delta_{1}=0.2 \delta\right)$, it appears that the numerical solutions obtained for Case 13 (grid $\delta \mathrm{x}=0.125 \Delta_{1}$ and FGR 8) and Case 14 (grid $\delta \mathrm{x}=0.0625 \Delta_{1}$ and FGR 16) almost match each other. This is consistent with Figures 4.2 (a) and 4.4. The turbulence intensities obtained from Case 11 ( $\delta \mathrm{x}=0.5 \Delta_{1}$ and FGR 2$)$ and Case $12\left(\delta \mathrm{x}=0.25 \Delta_{1}\right.$ and FGR 4$)$ are significantly different from the other two cases.

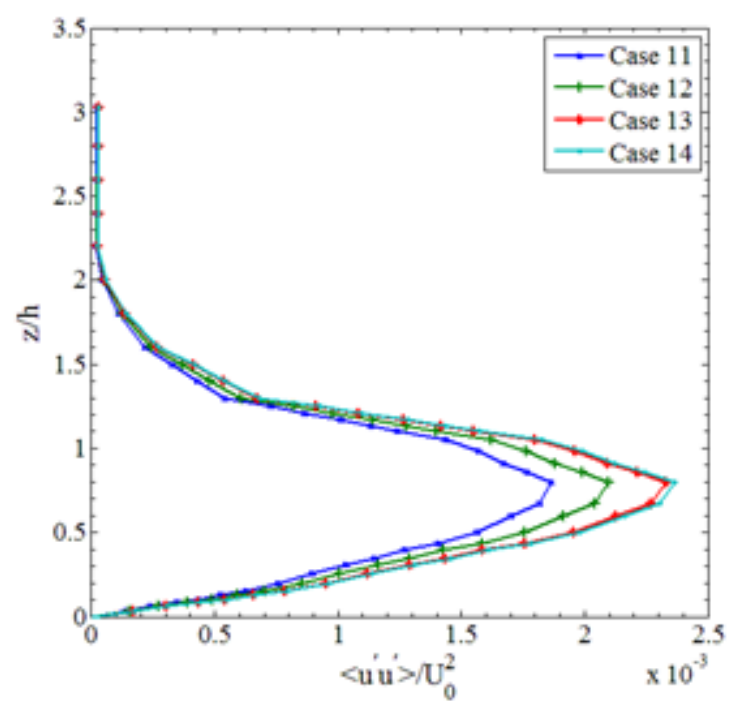

(a) At test section $x / h=4$

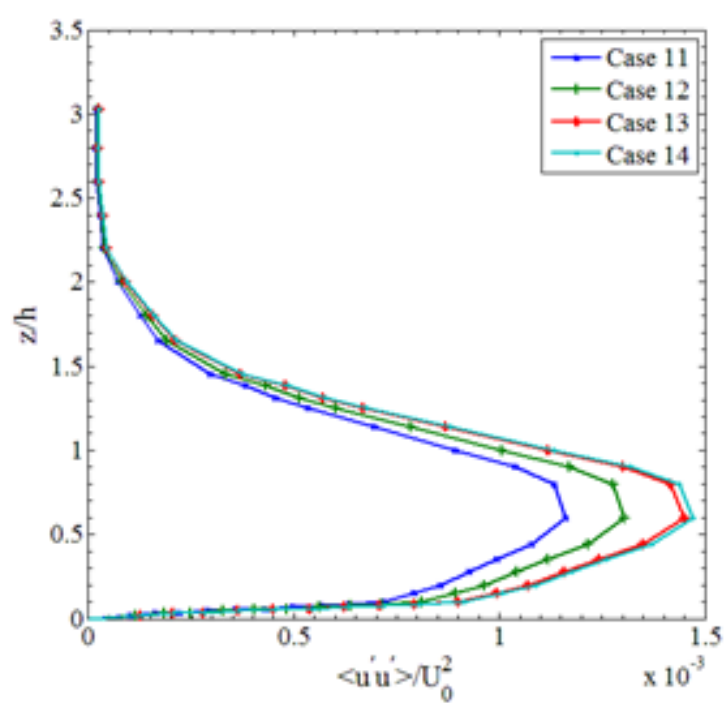

(c) At test section $x / h=10$

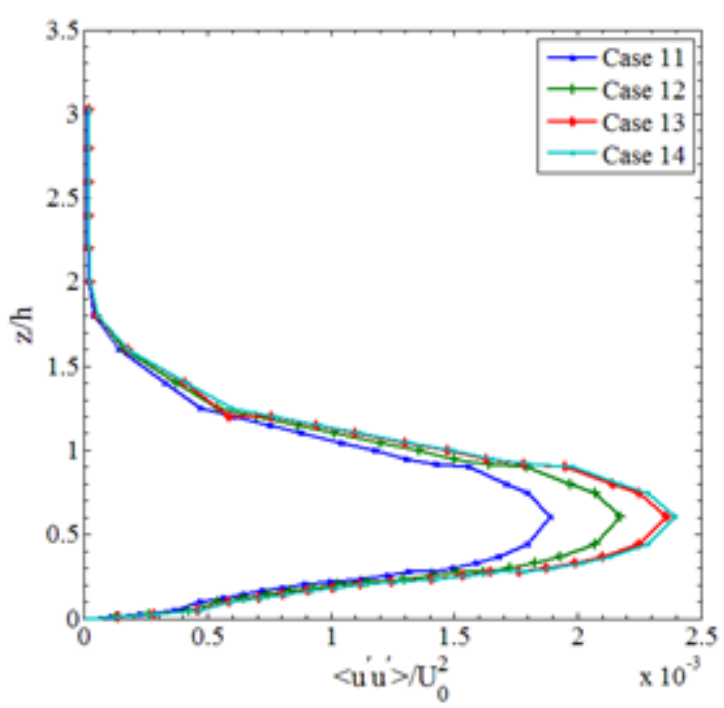

(b) At test section $x / h=6$

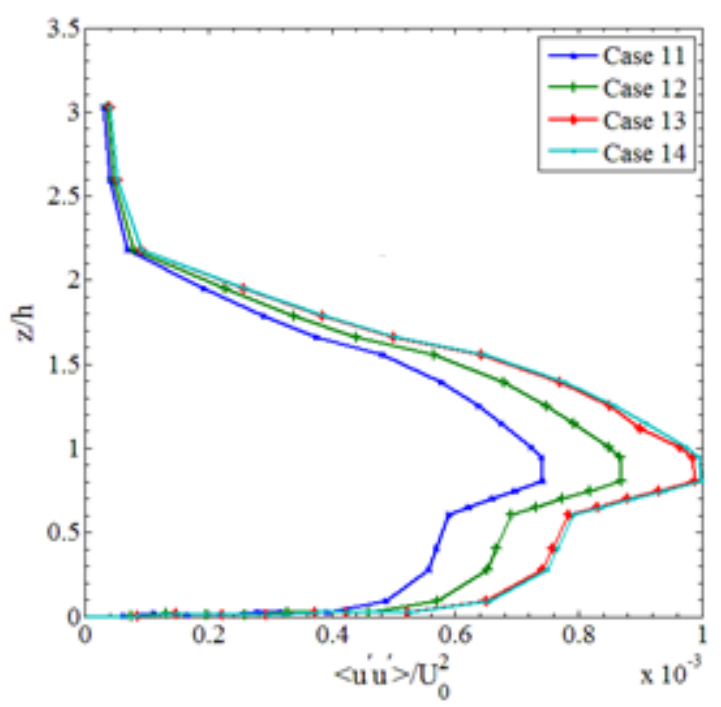

(d) At test section $x / h=19$

Figure 4.6. Turbulence intensities at selected test sections for fixed filter Case $1\left(\Delta_{1}=0.2 \delta\right)$ 
On the other hand, Figure 4.7 shows the reflection of outcome of energy spectra analysis on turbulence intensities for fixed filter width Case $2\left(\Delta_{2}=0.1 \delta\right)$. It can be observed that the turbulence intensity profiles obtained from Case $23\left(\delta \mathrm{x}=0.125 \Delta_{2}\right.$ and FGR 8) and Case 24 $\left(\delta \mathrm{x}=0.0625 \Delta_{2}\right.$ and FGR 16$)$ completely collapse onto each other. It also appears that the solution for Case $22\left(\delta \mathrm{x}=0.25 \Delta_{2}\right.$ and FGR 4$)$ shows some marginal difference compared to the converged solutions. This is consistent with Figures 4.2 (b) and 4.5.

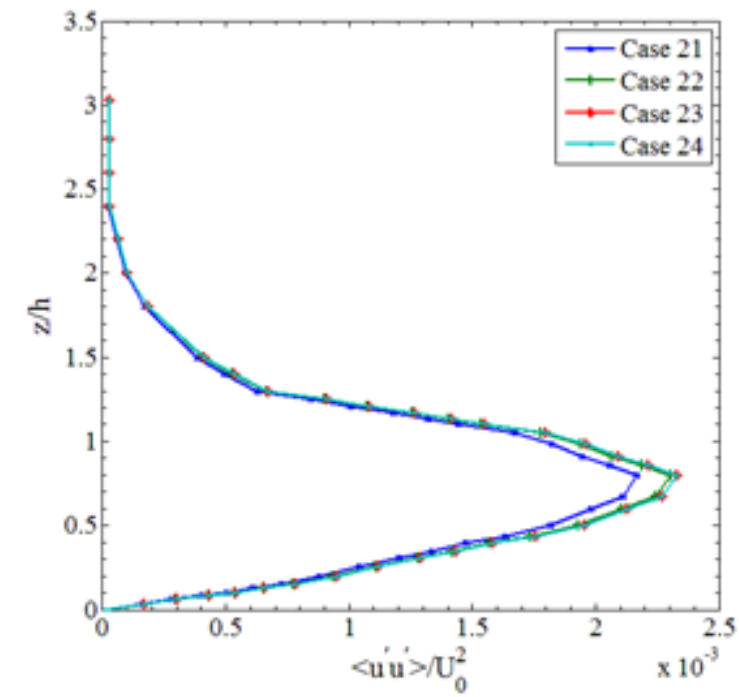

(a) At test section $x / h=4$

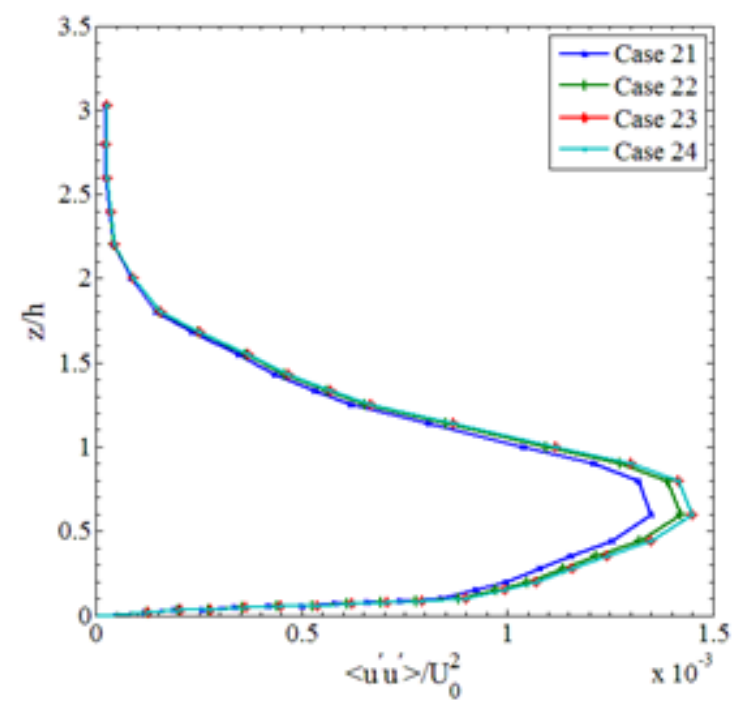

(c) At test section $x / h=10$

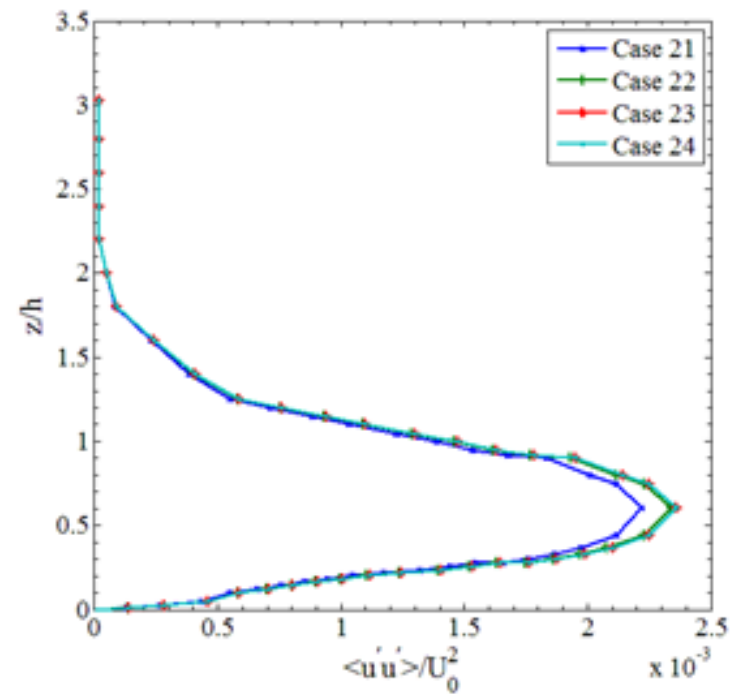

(b) At test section $x / h=6$

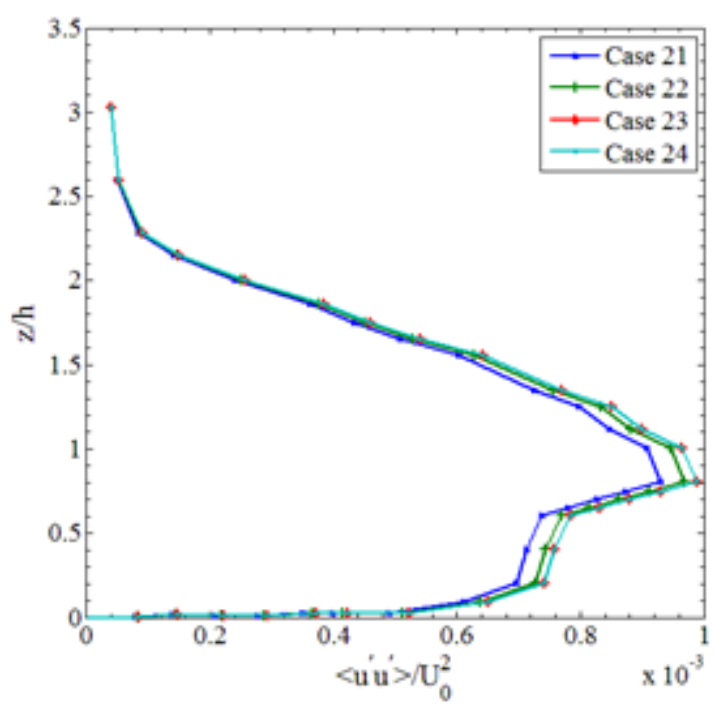

(d) At test section $x / h=19$

Figure 4.7. Turbulence intensities at selected test sections for fixed filter Case $2\left(\Delta_{2}=0.1 \delta\right)$ 


\subsubsection{Validation of the proposed model}

For each filter width case, the simulation results of the two finest resolutions are first compared graphically. Then the results are then compared with the experimental results of Jovic and Driver (1994) using statistical relative error analysis. For the latter, the method of Ierardi et al. (2003) has been followed which is discussed in the Chapter 3 .

\subsubsection{Graphical comparison}

Among the grid-converged solutions, mean normalised streamwise velocity and normalised streamwise velocity variance profiles generated in Case 13 and Case 23 are compared against experimental data first. Afterwards, the combinations of Cases 14 and 24, as the finest grid resolutions of each filter width cases, and Case 14 and 23 as the same grid resolutions but different filter width has been compared with the experimental results of both flow variables.

\section{Mean velocity}

\section{Comparison of coarser grid resolutions}

Mean normalised streamwise velocity profiles generated in Case $13\left(\delta \mathrm{x}=0.125 \Delta_{1}\right.$ and FGR 8$)$ and Case $23\left(\delta x=0.125 \Delta_{2}\right.$ and FGR 8$)$ are compared against experimental data in Figures 4.8. From the comparison it appears that predicted values are in good agreement with experimental data. From further observation, it also appears that Case 23 shows better qualitative agreement to experimental data compared with Case 13 at each of the selected locations. Overall the explicit LES predictions are in good agreement with the experimental data. As may be expected Case 23 with the finer explicit filter width better captures some of the finer details of the flow, more accurately predicting the peak recirculating mean velocity upstream of the reattachment point at $x / h=4$, and the transition of both the mean and variance at the outer edge of the boundary 
layer. The relatively small differences between the predictions for the two filter widths diminish downstream at $x / h=19$ where the complex flow structures due to the step have dissipated.

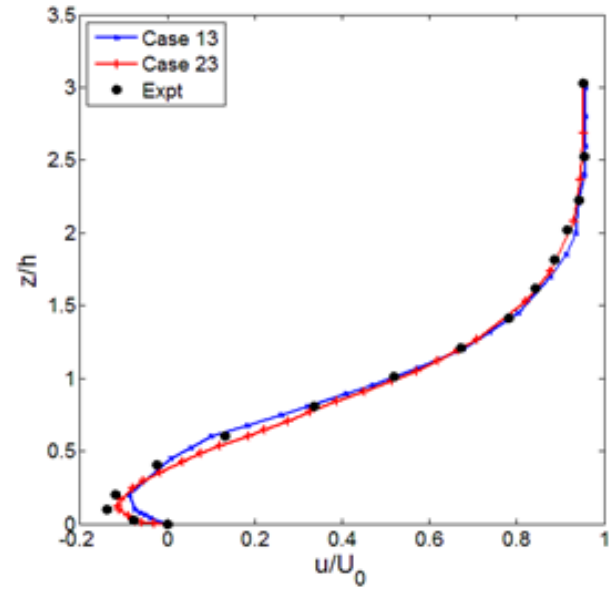

(a) At test section $x / h=4$

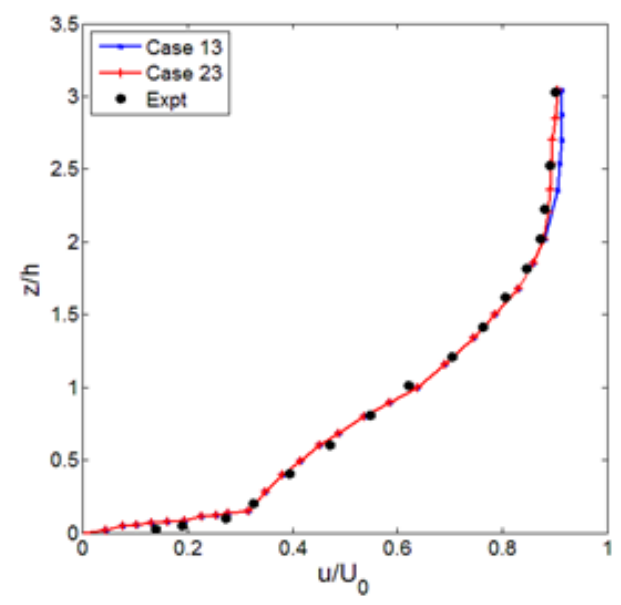

(c) At test section $x / h=10$

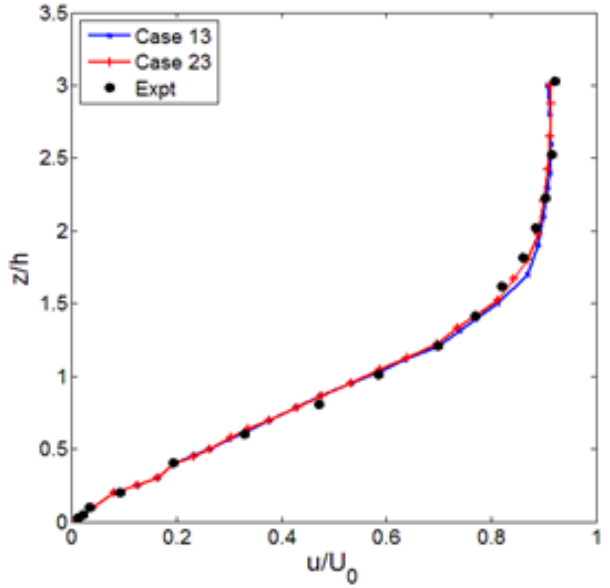

(b) At test section $x / h=6$

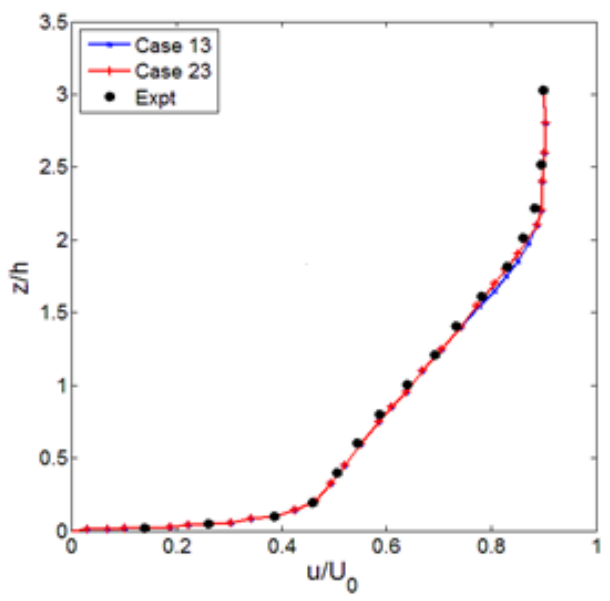

(d) At test section $x / h=19$

Figure 4.8. Mean velocity grid converged solution at relatively coarser grid resolution for fixed filter Case $1\left(\Delta_{1}=0.2 \delta\right)$ and Case $2\left(\Delta_{2}=0.1 \delta\right)$

\section{Comparisons at the finest grid resolutions}

Figure 4.9 shows the results for a comparison of the grid converged solutions with the finest grid resolutions of each of the fixed filter width cases. For Case 1 and Case 2, these are Case 14 $\left(\delta x=0.0625 \Delta_{1}\right.$ and FGR 16) and Case $24\left(\delta x=0.0625 \Delta_{2}\right.$ and FGR 16) respectively. From a comparison of the results, it appears that Case 24 shows better agreement to existing 
experimental data compared to Case 14 at all selected test locations of flow over a backward facing step, especially at $x / h=6$ in the range of $1.5<z / h<2$ and at $x / h=10$ above $z / h>2$.

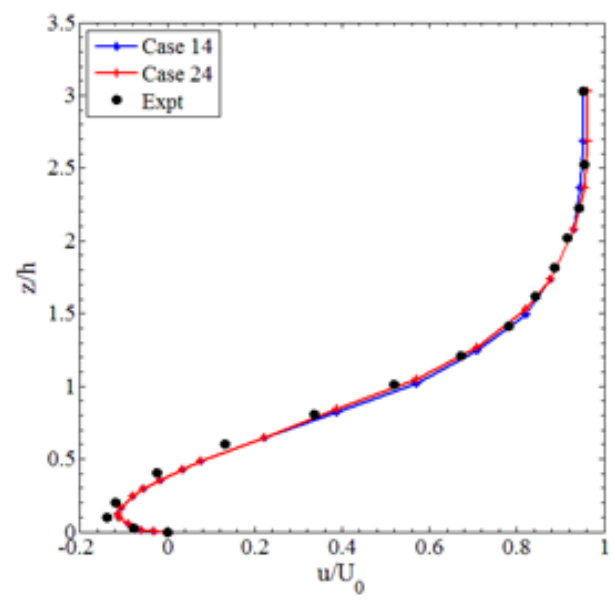

(a) At test section $x / h=4$

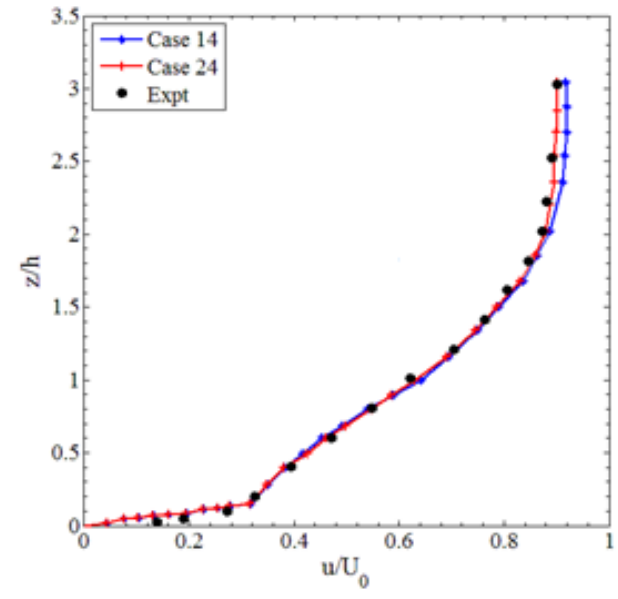

(c) At test section $x / h=10$

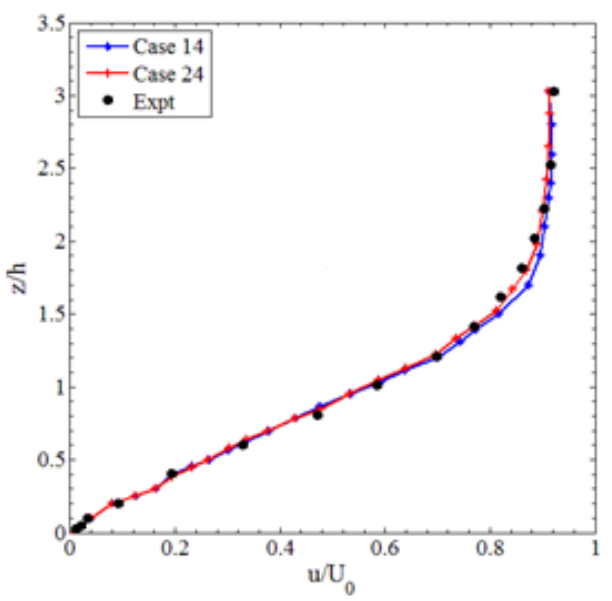

(b) At test section $x / h=6$

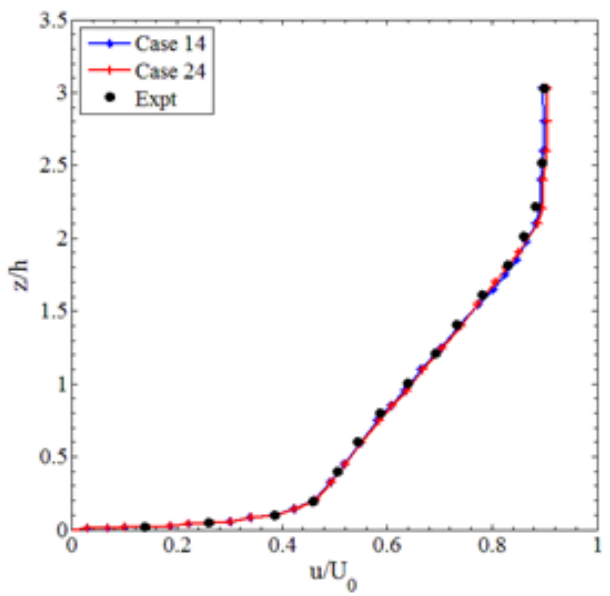

(d) At test section $x / h=19$

Figure 4.9. Mean velocity obtained with the finest grid resolution for fixed filter Case $1\left(\Delta_{1}=\right.$ $0.2 \delta)$ and Case $2\left(\Delta_{2}=0.1 \delta\right)$

\section{The effect of filter width}

Figure 4.10 shows a comparison of the grid-converged solutions of fixed filter Case 1 and Case 2 for the same grid resolutions at different filter width. Presented in Table 4.2, the same grid resolutions at different filter widths and filter to grid spacing ratios (FGR) are Case 14 
$\left(\delta \mathrm{x}=0.0625 \Delta_{1}\right.$ and FGR 16$)$ and Case $23\left(\delta \mathrm{x}=0.125 \Delta_{2}\right.$ and FGR 8$)$. From the comparison, it is found that Case 23 shows relatively better agreement to the experimental results compared to Case 14 at selected test sections. Moreover, it appears that for the same grid resolutions at different filter widths, relatively finer filter width is likely to provide more accurate numerical solutions.

Overall it can be observed that of the results presented in figures 4.8, 4.9 and 4.10 are consistent with the findings of figures 4.3(a), 4.3 (b) and 4.3(c), respectively.

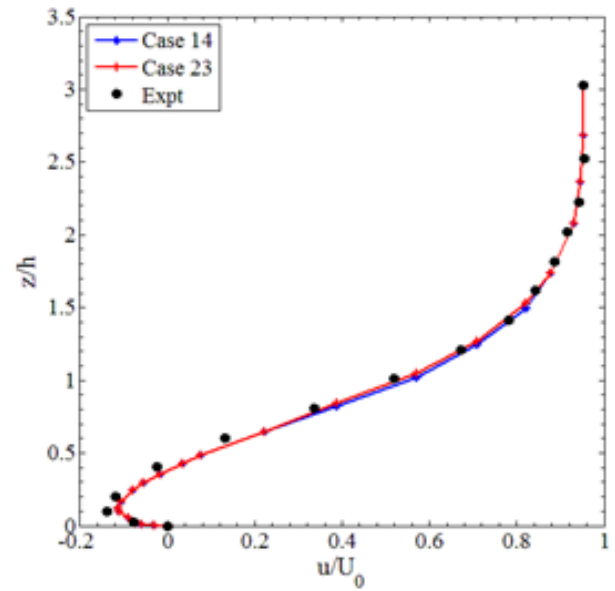

(a) At test section $x / h=4$

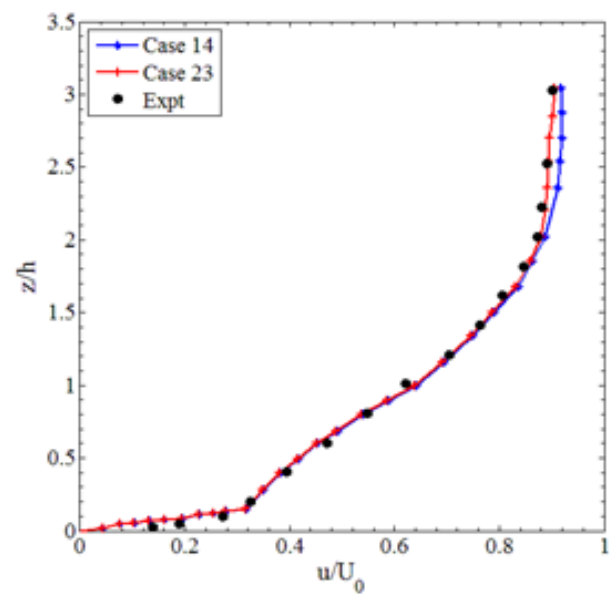

(c) At test section $x / h=10$

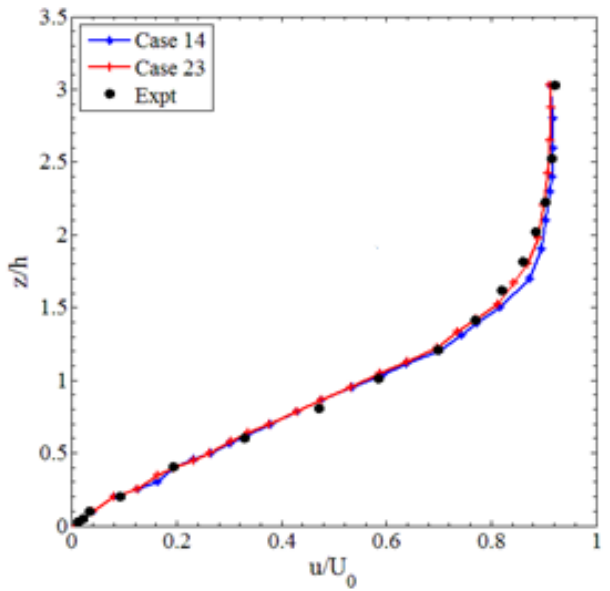

(b) At test section $x / h=6$

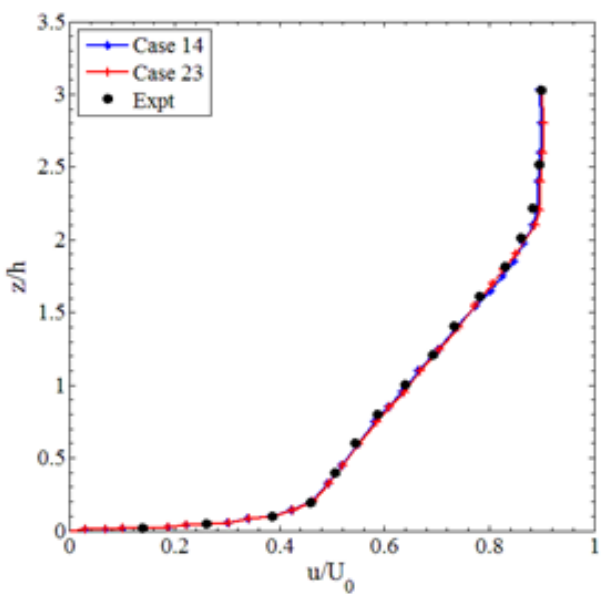

(d) At test section $x / h=19$

Figure 4.10. Mean velocity grid converged solution at same grid resolution for fixed filter Case $1\left(\Delta_{1}=0.2 \delta\right)$ and Case $2\left(\Delta_{2}=0.1 \delta\right)$ 


\section{Turbulence intensity}

\section{Comparison of coarser grid resolutions}

Figure 4.11 shows a comparison of the results for turbulence intensity in the same test locations for fixed filter Case 1 and Case 2. For the purpose of validation of results the fluctuations are normalized using the square of the upstream reference velocity. From the comparison of predicted turbulence intensity, it appears that Case $23\left(\delta \mathrm{x}=0.125 \Delta_{2}\right.$ and FGR 8$)$ is in reasonably good agreement with the experimental results compared to Case $13\left(\delta \mathrm{x}=0.125 \Delta_{1}\right.$ and FGR 8$)$ at

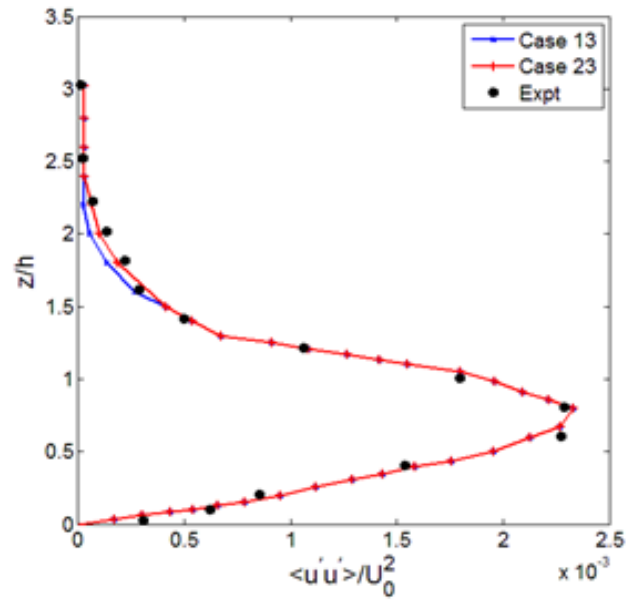

(a) At test section $x / h=4$

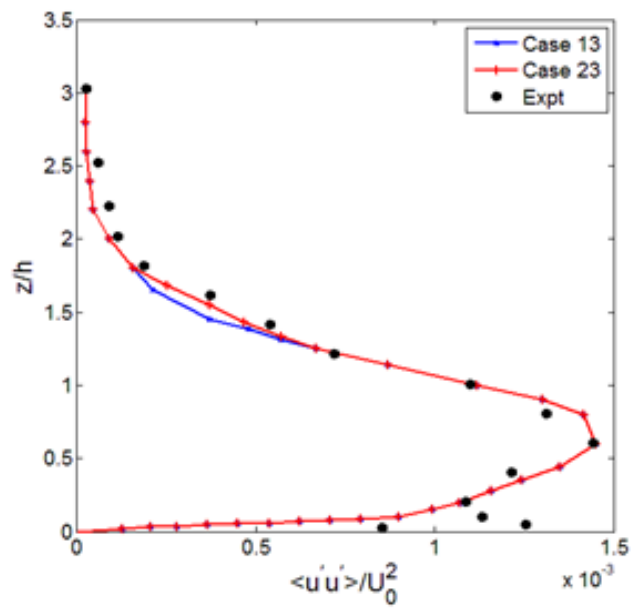

(c) At test section $x / h=10$

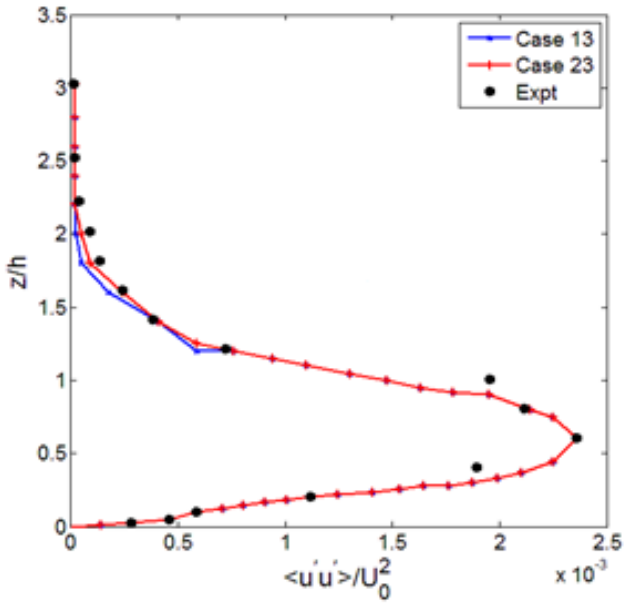

(b) At test section $x / h=6$

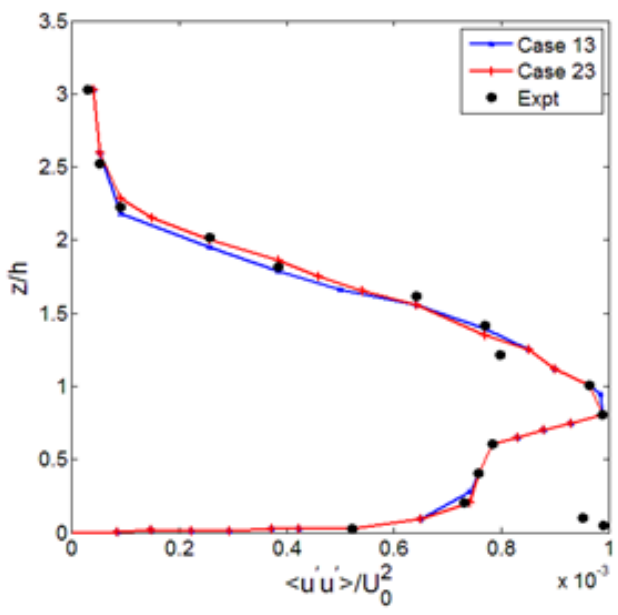

(d) At test section $x / h=19$

Figure 4.11. Grid converged solution of turbulence intensities at relatively coarse grid resolution for fixed filter Case $1\left(\Delta_{1}=0.2 \delta\right)$ and Case $2\left(\Delta_{2}=0.1 \delta\right)$ 
the test locations. For the selected filter widths neither of the explicitly filtered LES cases accurately predicts the near wall peak velocity variance at test sections $x / h=10$ and $x / h=19$. It is also noted that the DNS of Le, Moin and Kim (1997) did not accurately predict the near wall peak at $x / h=10$ but did agree well with the experimental data at $x / h=19$.

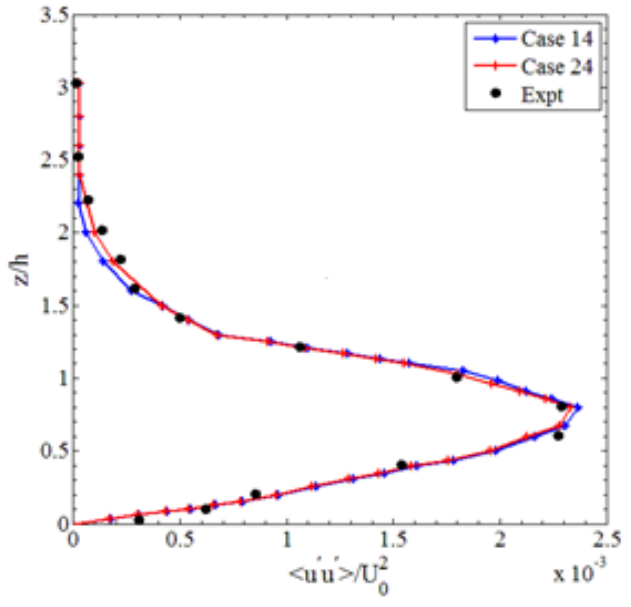

(a) At test section $x / h=4$

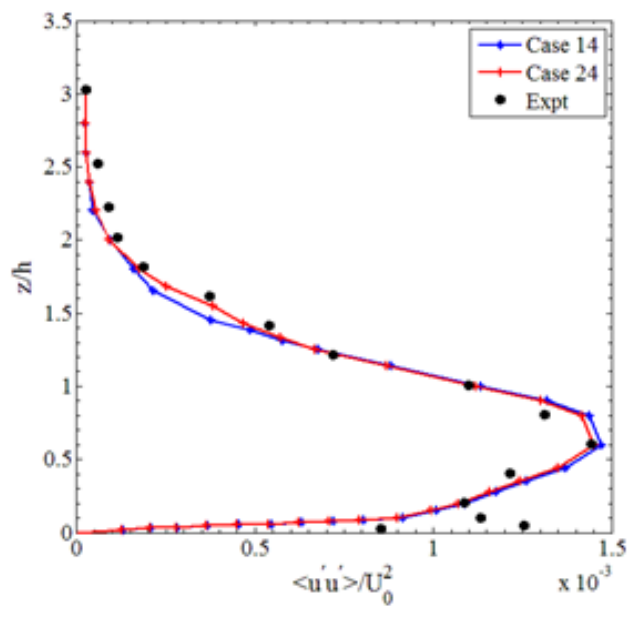

(c) At test section $x / h=10$

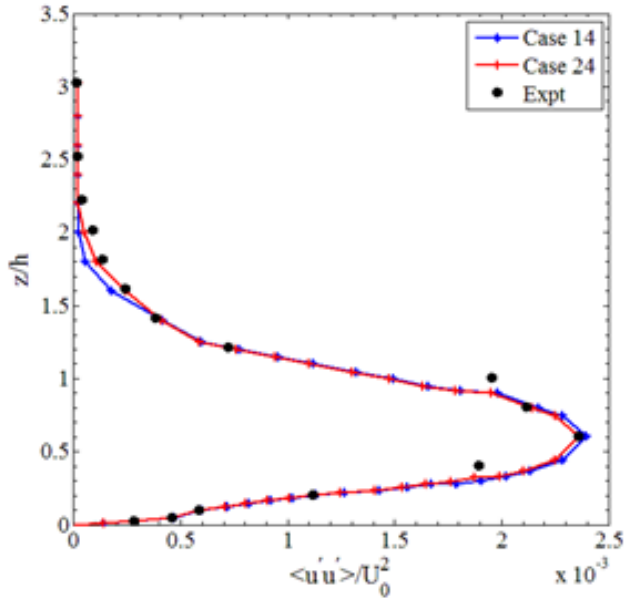

(b) At test section $x / h=6$

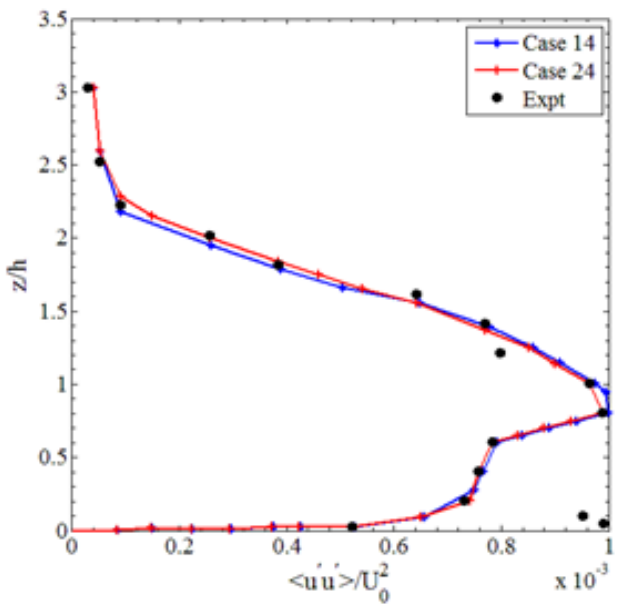

(d) At test section $x / h=19$

Figure 4.12. Turbulence intensities obtained with the finest grid resolution for fixed filter Case $1\left(\Delta_{1}=0.2 \delta\right)$ and Case $2\left(\Delta_{2}=0.1 \delta\right)$

\section{Comparisons at the finest grid resolutions}

Figure 4.12 shows the results for a comparison of grid-converged solutions of finest grid resolutions of fixed filter width cases for turbulent intensities at different test locations. The 
finest grid resolutions of filter Case 1 and Case 2 are Case $14\left(\delta x=0.0625 \Delta_{1}\right.$ and FGR 16) and Case $24\left(\delta x=0.0625 \Delta_{2}\right.$ and FGR 16) respectively. From a comparison of the results, it appears that Case 24 shows better agreement against existing experimental data compared to Case 14, at all selected test locations, particularly at $x / h=4$ and 19 approximately in the range of $1.5<$ $z / h<2.5$, at $x / h=6$ and $x / h=10$ approximately in the range of $1.4<z / h<2.2$ and $1.2<z / h<2$ respectively.

\section{The effect of filter width}

Figure 4.13 shows a comparison of the same grid resolutions at different filter widths for turbulence intensities at selected test locations for fixed filter width Case 1 and Case 2. The same grid resolutions at different filter width and filter to grid spacing ratios (FGR) are Case 14 $\left(\delta x=0.0625 \Delta_{1}\right.$ and FGR 16) and Case $23\left(\delta x=0.125 \Delta_{2}\right.$ and FGR 8). From the comparison, it is found that Case 23 shows relatively better agreement to the experimental results compared to Case 14, at different test sections. Moreover, it appears that for the same grid resolutions at different filter widths, relatively finer filter width is likely to provide more accurate numerical solutions for turbulence intensities at selected test sections.

Overall it can be observed that findings of Figures 4.11-13 are consistent with the findings of figure 4.3 .

\section{Summary of the validation exercise}

Overall, grid-converged solutions with filter width $\Delta_{2}$ taken as $10 \%$ of the BLT show comparatively better agreement over filter width $\Delta_{1}$ taken as $20 \%$ of BLT. It also appears that Case 23 with filter width $\Delta_{2} 10 \%$ of BLT and FGR 8 shows very good agreement to the existing reference data for mean velocity and turbulence intensity flow variables. 


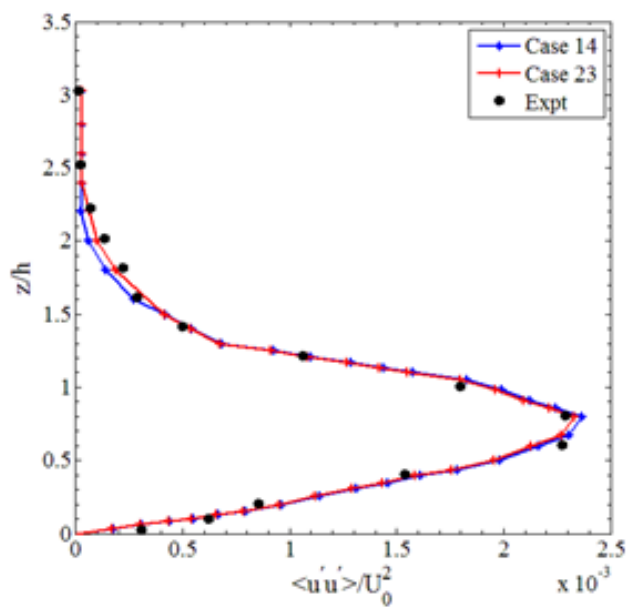

(a) At test section $x / h=4$

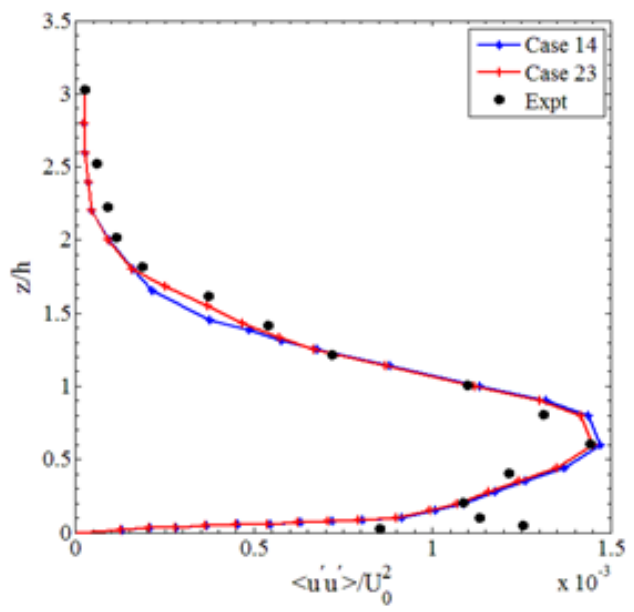

(c) At test section $x / h=10$

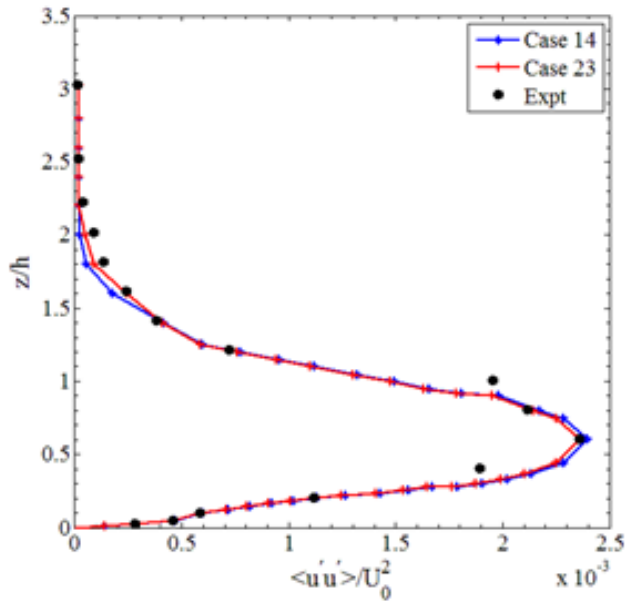

(b) At test section $x / h=6$

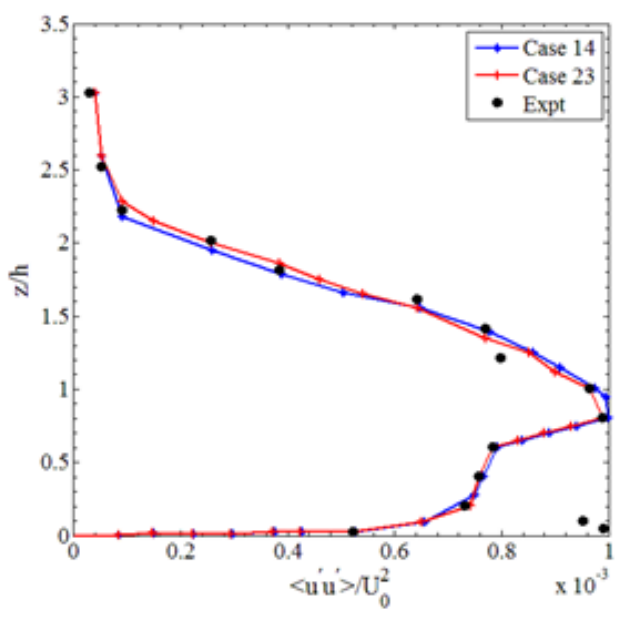

(d) At test section $x / h=19$

Figure 4.13. Grid converged solution of turbulence intensities at same grid resolution for fixed filter Case $1\left(\Delta_{1}=0.2 \delta\right)$ and Case $2\left(\Delta_{2}=0.1 \delta\right)$

\subsubsection{Statistical analysis of predicted outcomes}

Tables 4.3 and 4.4 show the cross-stream averaged relative errors of mean and variance of streamwise velocity for all grid-converged cases (i.e. Cases 13, 14, 23 and 24). Relative errors are calculated for each of the four streamwise locations and the simple average of those four stations. A number of interesting observations can be made. Firstly, the error levels are not excessively high considering the small number of sample points and depending on available computational resources even the most inaccurate of the cases (Cases 13) may be deemed 
acceptable (see more below). Secondly, as expected, due to filtering of high wave number fluctuations the velocity variance errors are generally higher than errors in the mean velocity.

Table 4.3. Cross-stream averaged relative error of mean streamwise velocity in grid-converged cases with different filter widths for flow over a backward facing step

\begin{tabular}{|c|c|c|c|c|}
\hline \multirow{2}{*}{$\begin{array}{c}\text { Test locations } \\
(x / h)\end{array}$} & \multicolumn{4}{|c|}{ Mean velocity } \\
\cline { 2 - 5 } & $\begin{array}{c}\text { Case 13 }(\%) \\
\delta \mathrm{x}=0.125 \Delta_{1}, \mathrm{FGR} 8\end{array}$ & $\begin{array}{c}\text { Case 14 }(\%) \\
\delta \mathrm{x}=0.0625 \Delta_{1}, \mathrm{FGR} 16\end{array}$ & $\begin{array}{c}\text { Case 23 }(\%) \\
\delta \mathrm{x}=0.125 \Delta_{2}, \mathrm{FGR} 8\end{array}$ & $\begin{array}{c}\text { Case 24 }(\%) \\
\delta \mathrm{x}=0.0625 \Delta_{2}, \mathrm{FGR} 16\end{array}$ \\
\hline 4 & 4.19 & 3.06 & 1.21 & 1.18 \\
\hline 6 & 2.79 & 1.29 & 1.25 & 1.12 \\
\hline 10 & 4.34 & 2.22 & 1.89 & 1.06 \\
\hline 19 & 3.73 & 1.13 & 0.67 & 0.93 \\
\hline Average & 3.76 & 1.93 & 1.26 & 256 \\
\hline Relative cost & 1 & 16 & 16 & \\
\hline
\end{tabular}

Table 4.4. Cross-stream averaged relative error of streamwise velocity variance in gridconverged cases with different filter widths for flow over a backward facing step

\begin{tabular}{|c|c|c|c|c|}
\hline \multirow{2}{*}{$\begin{array}{c}\text { Test locations } \\
(x / h)\end{array}$} & \multicolumn{4}{|c|}{ Turbulence intensity } \\
\hline & $\begin{array}{c}\text { Case } 13(\%) \\
\delta \mathrm{x}=0.125 \Delta_{1}, \mathrm{FGR} 8\end{array}$ & $\begin{array}{c}\text { Case 14 }(\%) \\
\delta \mathrm{x}=0.0625 \Delta_{1}, \text { FGR } 16\end{array}$ & $\begin{array}{c}\text { Case } 23(\%) \\
\delta \mathrm{x}=0.125 \Delta_{2}, \text { FGR } 8\end{array}$ & $\begin{array}{c}\text { Case 24 }(\%) \\
\delta \mathrm{x}=0.0625 \Delta_{2}, \text { FGR } 16\end{array}$ \\
\hline 4 & 4.65 & 3.89 & 3.43 & 2.56 \\
\hline 6 & 5.93 & 5.33 & 4.89 & 4.37 \\
\hline 10 & 6.19 & 5.78 & 5.41 & 5.13 \\
\hline 19 & 5.63 & 5.29 & 5.08 & 4.81 \\
\hline Average & 5.60 & 5.07 & 4.70 & 4.22 \\
\hline Relative cost & 1 & 16 & 16 & 256 \\
\hline
\end{tabular}

Relative error analysis allows a more precise comment on the trade-off between accuracy and computational cost than was possible in the comparison of the energy spectra. The final rows in Tables 4.3 and 4.4 indicate the approximate cost of the simulations relative to Case 13 which is the coarsest grid-converged simulation. This cost is estimated from the number of grid points and the allowable stable time step. Comparison of Cases 14 and 23 indicates that for a fixed computational cost the accuracy may be improved by reducing the filter size. This is especially so for the mean velocity which is improved considerably in Case 23 relative to Case 14 in the separated flow region just downstream of the step. The improvement in the accuracy of velocity variance predictions ( $r m s$ profiles of the velocity fluctuations normalised by $U_{0}^{2}$ ) is also apparent but smaller in magnitude due to it being a squared quantity. Case 24 is 16 times more 
computationally expensive than Cases 14 and 23 yet achieves only a relatively modest improvement in accuracy largely because the filter width remains unchanged relative to Case 23 and the near wall structures are not fully captured. If resources permit greater computational load, one would be better off reducing the filter width by half while maintaining FGR at 8 . In fact with finer filters it may be possible to achieve grid convergence with even lower FGR thus not increasing the cost as much as might otherwise be expected (c.f. Figure 4.2(b) where Case 22 with FGR = 4 is nearly, but not quite, grid converged). From a computational cost point of view,

Case 24 requires sixteen times higher computational cost than Case 23, apart from having a reduced need for temporal resolution. Moreover averaged relative error for two flow variables are within within 5\% which is good as per Roache (1998) and Roy (2003). Therefore, it can be considered as a practically viable case.

From the overall statistical error analysis, it considered that obtained numerical results for both flow variables at selected test locations are in good agreement with experiment data. It is to be noted that in LES, the real velocity is not calculated, rather it is the filtered velocity. The simulations for one filter width is expected to converge to the real solution filtered with that filter width. Therefore, it is expected that Cases 14 and 24, being the converged solutions at two different filter widths, do not give the same results. If we compare Case 14 and Case 24 in Figures 4.9 (especially $\mathrm{b}$ and c) and Table 4.3 we can see slight differences as expected. Comparing quantitative averaged mean errors Case 14 has $1.93 \%$ a compared to $1.07 \%$ of Case 24.

\subsubsection{Comparison between simulation with implicit and explicit filtered LES}

The computational domains of Chapter 4 and Chapter 3 are same, but the physical resolutions are different due to the grid selection procedure. In Chapter 3 grids are based on step height to grid 
spacing ratio $(h / \delta z)$. On the other hand, grids in Chapter 4 are based on a certain percentage of the BLT.

Table 4.5. Comparison of relative error of streamwise velocity and velocity variance between implicit and explicit filtered LES

\begin{tabular}{|c|c|c|c|c|c|c|c|c|c|c|c|c|c|}
\hline \multirow{4}{*}{ Grids } & \multirow{4}{*}{$\begin{array}{l}\text { Test } \\
\text { sec. } \\
(x / h)\end{array}$} & \multirow{2}{*}{\multicolumn{3}{|c|}{$\frac{\text { Implicit }}{\text { Very fine }(h / \delta z=20)}$}} & \multicolumn{9}{|c|}{ Explicit } \\
\hline & & & & & \multicolumn{3}{|c|}{ Case $14\left(\Delta_{1}=0.2 \delta\right)$} & \multicolumn{3}{|c|}{ Case $23\left(\Delta_{2}=0.1 \delta\right)$} & \multicolumn{3}{|c|}{ Case $24\left(\Delta_{2}=0.1 \delta\right)$} \\
\hline & & \multirow{2}{*}{$n^{+}$} & \multicolumn{2}{|c|}{ Relative error (\%) } & \multirow{2}{*}{$n^{+}$} & \multicolumn{2}{|c|}{ Relative error (\%) } & \multirow{2}{*}{$n^{+}$} & \multicolumn{2}{|c|}{ Relative error (\%) } & \multirow{2}{*}{$n^{+}$} & \multicolumn{2}{|c|}{ Relative error (\%) } \\
\hline & & & Mn. Vel. & Trb. Int. & & Mn. Vel. & Trb. Int. & & Mn. Vel. & Trb. Int. & & Mn. Vel. & Trb. Int. \\
\hline \multirow{2}{*}{$480 \times 160 \times 320$} & 4 & & & & \multirow{2}{*}{$\leq 0.93$} & 3.06 & 3.89 & \multirow{2}{*}{$\leq 0.79$} & 1.21 & 3.43 & & & \\
\hline & 6 & & & & & 1.29 & 5.33 & & 1.25 & 4.89 & & & \\
\hline \multirow{2}{*}{$600 \times 240 \times 400$} & 4 & \multirow{2}{*}{$\leq 0.46$} & 4.38 & 6.58 & & & & & & & & & \\
\hline & 6 & & 5.73 & 7.12 & & & & & & & & & \\
\hline \multirow{2}{*}{$960 \times 320 \times 640$} & 4 & & & & & & & & & & \multirow{2}{*}{$\leq 0.43$} & 1.18 & 2.56 \\
\hline & 6 & & & & & & & & & & & 1.12 & 4.37 \\
\hline
\end{tabular}

For implicit LES solution of very fine resolution (VF) with $h / \delta z=20$, filter width $\Delta=0.02 \delta$ and $\mathrm{FGR}=1$ from Chapter 3 is taken to compare with explicitly filtered LES solution whose grid resolution lies between the resolution of Case 14 with filter width $\Delta=0.2 \delta$, Case 23 and Case 24 with filter width $\Delta=0.1 \delta$. Table 4.5 presents the comparison of relative error of streamwise mean velocity and turbulence intensity at test sections $x / h=4$ and $x / h=6$ between implicit and explicit filtered LES. From the table, it appears that explicit Case 14 and 23, despite having higher nominal $n^{+}$at relatively coarse grid than implicit case with VF resolution, provides quite good agreement with experimental data and relative errors are within acceptable range of 5\% (Roache 1998, Roy 2003) at selected test locations for both of the flow variables. It is also found that although $n_{\min }^{+}$of implicit VF is almost similar to the explicit Case 24, but the Case 24 provides much better agreement with the experimental data compared to the VF. The comparison between this two schemes shows explicit LES performs much better than implicit LES which clearly demonstrate the advantage of using explicitly filtered LES over implicit LES. 


\subsubsection{Prominent features of the flow}

Figure 4.14 presents the instantaneous streamwise velocity at $12 \mathrm{~s}$ along the midsection of the high Reynolds number flow over a backward facing step. Results are for Case 23 which is the finer filter width case with $F G R=8$. The simulation exhibits the major features expected in such a flow, with the separation region downstream of the step, the reattachment point and redeveloping boundary layer clearly evident. The reattachment length from instantaneous filtered velocity for Case 23, calculated according to the method of Le, Moin and Kim (1997) is $X_{r}=$ $6 h \pm 0.21 h$ which compares very well with the experimentally observed length of $6 h \pm 0.15 h$. The predicted reattachment length for Case 13 with the coarser filter width $\left(\Delta_{1}=0.2 \delta\right)$ is also acceptably good although slightly less accurate at $X_{r}=6 h \pm 0.28 h$.

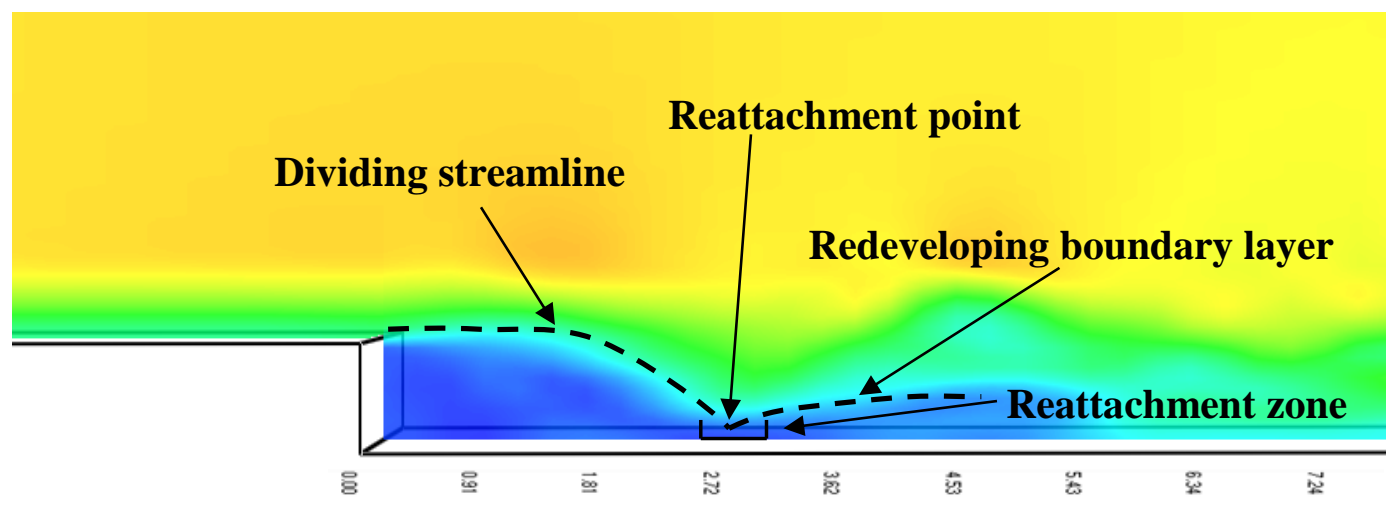

$\operatorname{vel}(\mathrm{m} / \mathrm{s})$

Figure 4.14. Instantaneous streamwise velocity at $12 \mathrm{~s}$ along the midsection of the high Reynolds number flow over a backward facing step. Results are for Case 23

\subsection{Conclusions}

Flow over a backward facing step is a widely used benchmark case for its prominent flow features such as flow separation, reattachment, and redevelopment of the boundary layer. In this study, this benchmark case is considered as having a high Reynolds number turbulent flow with $R e_{\lambda} 115$. A systematic approach to explicitly filtered large eddy simulations (LES) is used to simulate this flow case. Two filter widths $\Delta_{1}$ and $\Delta_{2}$ are selected as $10 \%$ and $20 \%$ of the 
boundary layer thickness respectively. While conducting the energy spectra analysis, focus is given on the necessity to consider the fact that whether selected filter widths $\Delta_{1}$ and $\Delta_{2}$ fulfil the criteria for satisfying the principle of LES by capturing the energy containing lengthscales of eddies. The cases with the finer filter width (Case 23 and Case 24) are more accurate than the cases with the coarser filter (Case 13 and 14) and increasing the FGR from 8 to 16 (i.e. comparing Case 13 with Case 14 and Case 23 with 24) leads to a reduction in the errors but less so for the finer filter width cases. It indicates that although deemed grid-converged from the perspective of the energy spectra some grid-sensitivity remains, albeit small and diminishing with refinement of the grid. From the energy spectrum analysis and comparison with experimental results, it appears that filter width taken as $10 \%$ of the BLT with filter to grid spacing ratio (FGR) 8 is more likely to provide an appropriate solution in an explicit scheme with optimal computational resources. It satisfies the LES principle by capturing the energy containing eddies. Moreover, it shows very good agreement with the $-5 / 3$ universal theoretical slope that is proportional to wavenumbers $\kappa^{-5 / 3}$ of energy spectra and the taken filter width lies within the inertial sub range. This study also shows that at sufficiently high Reynolds number turbulent flows the inertial subrange develops and clearly exists in the energy spectra. Furthermore, simulations outputs of other flow variables such as the mean velocity and turbulence intensities of the benchmark case show very good agreement with experimental data at selected test locations. This justifies the important fact that a filter width taken as $10 \%$ of the BLT with FGR 8 is adequate to obtain appropriate LES solutions using the explicit scheme for the test case considered. Comparison between the two LES schemes (implicitly and explicitly filtered) shows the explicitly filtered LES performs much better than the implicitly filtered LES at coarser grid resolution and the obtained numerical results with explicit LES are within the 
acceptable range of 5\%. Additionally, the prominent flow features of Case 23 show that the explicit scheme captures all the essential physics of the flow. Overall, this study demonstrates that an appropriate choice of filter width and grid resolution can be used to obtain numerically converged solution in the case of high Reynolds number flow. 


\section{Chapter 5}

\section{Low Reynolds number turbulent flow}

Thus far, we have demonstrated that explicit filtering is highly effective for turbulent flows that exhibit a clearly defined inertial range. However, it is important to investigate the effectiveness of explicit filtering in flow regions in which turbulence is not fully established in the sense that an inertial subrange is not so distinct. Such flows are observed in differentially heated cavities. We have demonstrated that an appropriate choice of LES filter width and grid resolution can be used to obtain numerically converged solutions in the case of high Reynolds number flows. We now turn our attention to a low Reynolds number turbulent flow.

\subsection{Studied numerical configuration}

Numerical and experimental studies in differentially heated cavities are a fruitful source of data on turbulent buoyancy driven flows. Here such a flow is used as our low Reynolds number test case. The computational domain conforms precisely to that of the experimental set up of Cheesewright et al. (1986). Figure 5.1 shows a schematic of the configuration indicating the hot and cold vertical walls and the remaining surfaces which are adiabatic. A detailed picture is given of the flow in the vicinity of the heated wall where both velocity and temperature boundary layers develop due to buoyancy effects. The schematic also shows the flow direction of the fluid and the development of anti-symmetric boundary layers driven by the temperature differential leading to an upward flow direction near the heated wall and downward flow direction near the cold wall. Due to changes in shear stress along the walls of the cavity turbulence occurs with different ranges of length and time scales, and this provides a useful test of the explicitly filtered LES method developed in this study. 
The temperatures of the hot and cold walls are constant and uniform with the hot wall maintained at $77.2^{\circ} \mathrm{C}$ whereas the cold wall temperature is maintained at $31.4^{\circ} \mathrm{C}$. The thermal diffusivity and volumetric expansion coefficient of the fluid, which is air, are $2.27 \times 10^{-5} \mathrm{~m}^{2} / \mathrm{s}$ and $3.30 \times$ $10^{-3} \mathrm{~K}^{-1}$, respectively. The dimensions of the cavity are $x \times y \times z=0.5 \mathrm{~m} \times 1.0 \mathrm{~m} \times 2.5 \mathrm{~m}$.

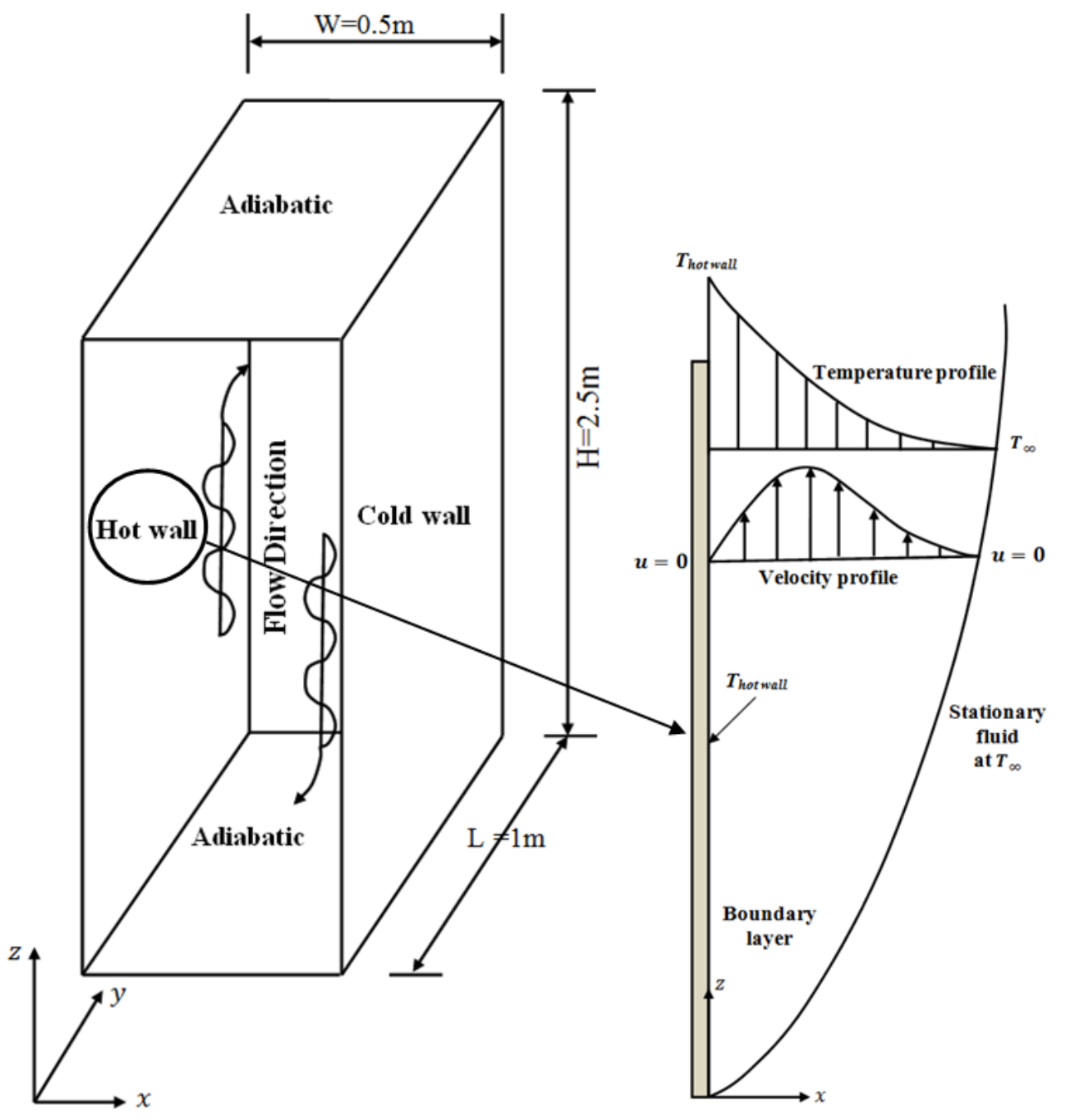

Figure 5.1. Schematic of buoyancy driven flow inside a differentially heated rectangular cavity with particular emphasis is given to boundary layer development near the hot wall In this present study $R e_{\lambda}$ is found to be approximately 25 and the flow is therefore considered as having a low Reynolds number (Sreenivasan 1994). Unlike the momentum driven flow over the backward facing step, the cavity flow is buoyancy driven and transition to turbulence is 
customarily characterised by the Rayleigh number $(R a)$. It is useful insofar as it indicates whether conduction is dominant over convection, or vice versa. In this study, the Rayleigh number $(R a)$ in the simulations is $4.68 \times 10^{10}$, the same as the experiment by Cheesewright et al. (1986).

Table 5.1. Filter sizes $\Delta_{1}$ and $\Delta_{2}$ for a turbulent flow at low Reynolds numbers

\begin{tabular}{|c|c|c|c|c|}
\hline \multicolumn{2}{|c|}{ Case 1 } & \multicolumn{2}{|c|}{ Case 2 } & $\begin{array}{c}\text { Filter/grid } \\
\text { ratio }\end{array}$ \\
\hline Fixed Filter width $\Delta_{1}=0.2 \delta$ & \multicolumn{2}{|c|}{ Fixed Filter width $\Delta_{2}=0.1 \delta$} & $\Delta / \delta \mathrm{x}$ \\
\hline Case 11 & $\delta \mathrm{x}=0.5 \Delta_{1}$ & Case 21 & $\delta \mathrm{x}=0.5 \Delta_{2}$ & 2 \\
\hline Case 12 & $\delta \mathrm{x}=0.25 \Delta_{1}$ & Case 22 & $\delta \mathrm{x}=0.25 \Delta_{2}$ & 4 \\
\hline Case 13 & $\delta \mathrm{x}=0.125 \Delta_{1}$ & Case 23 & $\delta \mathrm{x}=0.125 \Delta_{2}$ & 8 \\
\hline Case 14 & $\delta \mathrm{x}=0.0625 \Delta_{1}$ & Case 24 & $\delta \mathrm{x}=0.0625 \Delta_{2}$ & 16 \\
\hline
\end{tabular}

\section{Boundary conditions}

For the numerical set up of the test case, a no-slip boundary condition was set for the velocities at the solid walls. For the temperature, a Dirichlet boundary condition was applied to the hot $\left(T_{H}=77.2^{\circ} \mathrm{C}\right)$ and cold $\left(T_{C}=31.4^{\circ} \mathrm{C}\right)$ walls. The top and the bottom walls are considered to be adiabatic, Neumann boundary condition was invoked for the temperature, i.e. $\partial T / \partial n=0$, where $n$ is representing the perpendicular direction to the wall.

\section{Filter to grid spacing ratio (FGR)}

In this study filter to grid spacing ratios (FGRs) of 2, 4, 8 and 16 are used for each filter width. Table 5.1 shows that a total of eight simulations need to be performed for two filter widths that are selected as $10 \%$ and $20 \%$ of the boundary layer thickness (BLT). The BLT of flows past a heated vertical plate can be estimated using an analytical expression based on the Grashof $\left(G r_{z}\right)$ and Prandtl (Pr) numbers (Holman 2004),

$$
\frac{\delta}{Z}=3.93 \operatorname{Pr}^{-\frac{1}{2}}(0.952+\operatorname{Pr})^{1 / 4} G r_{z}^{-1 / 4}
$$


where $z$ is the streamwise distance downstream from the start of the boundary layer, the Prandtl number $(P r)$ is taken to be 0.7 and the Grashof number is defined by,

$$
G r_{z}=\frac{g \beta}{v^{2}}\left(T_{s}-T_{\infty}\right) z^{3}
$$

where, $g$ is the acceleration due to gravity $\left(9.81 \mathrm{~m} / \mathrm{s}^{2}\right), \beta$ is the thermal expansion coefficient $\left(1 / T_{\infty}\right), v=1.8 \times 10^{-5} \mathrm{~kg} / \mathrm{ms}$ is the kinematic viscosity of the fluid, $T_{S}$ is the surface temperature, $T_{\infty}$ is the ambient temperature. In the absence of a similar expression for flows in differentially heated cavities, above correlations are used and the characteristic BLT is calculated at the mid-height of the cavity. The analytically estimated typical BLT at the mid-height in the cavity flow is $\delta \leq 7.8 \times 10^{-2} \mathrm{~m}$ which compares favourably with $\delta \leq 8.5 \times 10^{-2} \mathrm{~m}$ measured by Cheesewright et al. (1986).

Table 5.2. Grid resolutions for a buoyancy driven differentially heated cavity flow at a low Reynolds number, $R e_{\lambda}=25$

\begin{tabular}{|c|c|c|c|}
\hline \multicolumn{4}{|c|}{$\begin{array}{c}\text { Row Reynolds number turbulent flow inside a Cavity } \\
\left.\text { (Estimated Reynolds number, } R e_{\lambda}=25\right)\end{array}$} \\
\hline Test cases & $N_{x} \times N_{y} \times N_{z}$ & $n_{\text {hot Wall }}^{+}$ & $n_{\text {Cold Wall }}^{+}$ \\
\hline Case 11 & $40 \times 20 \times 100$ & $\leq 4.52$ & $\leq 5.22$ \\
\hline Case 12 & $80 \times 40 \times 200$ & $\leq 2.95$ & $\leq 3.15$ \\
\hline Case 13 & $160 \times 80 \times 400$ & $\leq 1.04$ & $\leq 1.16$ \\
\hline Case 14 & $320 \times 160 \times 800$ & $\leq 0.66$ & $\leq 0.73$ \\
\hline Case 21 & $80 \times 40 \times 200$ & $\leq 2.18$ & $\leq 2.31$ \\
\hline Case 22 & $160 \times 80 \times 400$ & $\leq 0.88$ & $\leq 0.98$ \\
\hline Case 23 & $320 \times 160 \times 800$ & $\leq 0.52$ & $\leq 0.63$ \\
\hline Case 24 & $640 \times 320 \times 1600$ & $\leq 0.21$ & $\leq 0.34$ \\
\hline
\end{tabular}

The rationale behind the filter selection as a certain proportion of BLT has been described in the earlier part of the Chapter 2. Here, for fixed filters $\Delta_{1}$ and $\Delta_{2}$, the filter width is taken as twice of the grid size (considered as a coarse mesh), and this is the baseline case. We used a FGR of 2 for 
the baseline cases because this ratio seems to be most commonly adopted in recently published studies (Gullbrand 2002, Gullbrand and Chow 2003, Radhakrishnan and Bellan 2012, 2013 and 2015). Each fixed filter FGR is then refined progressively by 4, 8 and 16 times to obtain a gridconverged solution.

\section{Grid resolutions of flow inside a rectangular cavity}

Table 5.2 presents the grid resolutions for buoyancy driven rectangular cavity flow taken for two selected fixed filter widths Case $1\left(\Delta_{1}=0.2 \delta\right)$ and Case $2\left(\Delta_{2}=0.1 \delta\right)$ which are taken as $20 \%$ and $10 \%$ of the boundary layer thickness $(\delta)$ respectively. $N_{x}, N_{y}$ and $N_{z}$ represents the number of grids (or meshes) along the wall normal $\left(L_{x}\right)$, spanwise $\left(L_{y}\right)$ and streamwise $\left(L_{z}\right)$ length directions. A rectangular grid spacing was used in all simulations. Since buoyancy driven cavity flow consists of complex flow patterns near the walls, the grid was refined as $0.5,0.25,0.125$ and 0.0625 of the filter widths for Case 1 and Case 2 (refer to Table 5.1 and 5.2) in the vicinity of the wall surfaces up to $2 \delta$. In the domain beyond $2 \delta$ the cell sizes were increased normal to the horizontal and vertical directions. The expansion ratio is 2.

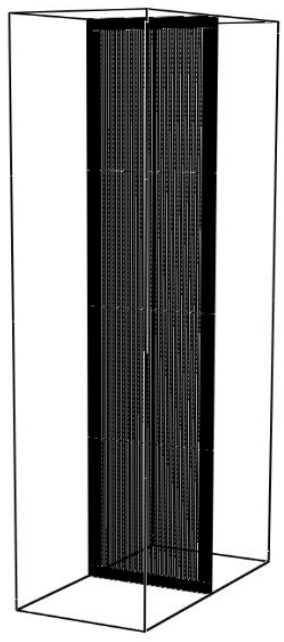

(a)

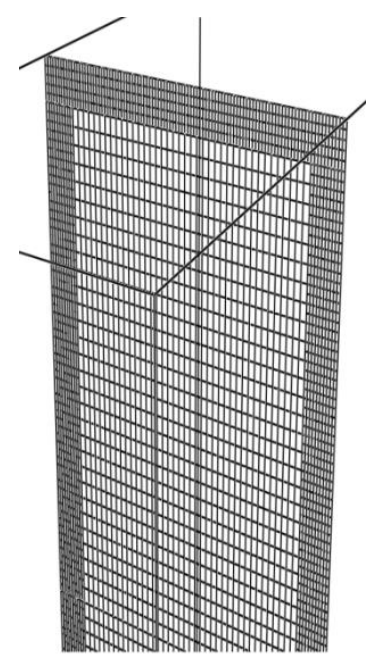

(b)

Figure 5.2. Schematic diagram of grid resolutions for (a) Case 12 (b) Enlarged view near the top wall of rectangular cavity 
The nominal dimensionless wall units $n_{\min }^{+}$of the grid points closest to the walls are also presented in Table 5.2. Figure 5.2(a) presents the schematic of the complete computational domain and grid resolutions of Case $12(80 \times 40 \times 200)$ (from Table 5.2) and Figure 5.2(b) presents the enlarged version of partial configuration near the top wall to show the grid arrangement more clearly. The total simulation time for the buoyancy driven cavity case was set at 300s. The flow achieved statistically-steady state at approximately $300 t_{E}$ where unit turnover time is $1.7 \times 10^{-1} \mathrm{~s}$ considering the maximum BLT as same size as the largest eddy (Tennekes and Lumley 1972). After an initial run of $300 t_{E}$, the flow velocity and temperature is averaged over the time for another $1500 t_{E}$. The average velocity data was obtained at $0.01 \mathrm{~s}$ interval (nearly one eddy turnover time in each interval) and statistical average of 25000 data points was taken which is considered adequate.

\subsection{Results and discussions}

\subsubsection{Energy spectra analysis}

An important feature of buoyant flow in an enclosure is that flow evolves continuously along the walls due to the effect of shear stress and convective heat transfer. Furthermore, depending on the Rayleigh number $(R a)$, the flow can be laminar or turbulent. Paolucci et al. (1989) report that the transition from laminar to turbulent flow occurs at Rayleigh number between $10^{8}$ and $10^{9}$ and increasing the $R a$ renders the flow increasingly turbulent. The $R a$ in this study is considered $4.68 \times 10^{10}$. Moreover, due to wall shear stress turbulence occurs at different ranges of length and time scales in the flow. For these reasons, the flow field is quite complex in the cavity flow. In this section results are analysed based on energy spectra to ensure that the results obtained from cavity flow which is considered as low Reynolds number flow are consistent with the proposed approach in the explicit scheme.

Figure 5.3 shows the grid-convergence of the three-dimensional energy spectra obtained for fixed filter Case $1\left(\Delta_{1}=0.2 \delta\right)$ and Case $2\left(\Delta_{2}=0.1 \delta\right)$. The Kolmogorov's theoretical $-5 / 3$ scaling 
for the inertial sub-range is observed tangential to the energy spectra. The beginning of the inertial subrange is shown along with short vertical dotted lines separating the energy containing and inertial ranges. Vertical lines have also been included to locate the nominal cut-off wave number corresponding to the filters $\left(\kappa_{c}=\pi / \Delta\right)$. From the figure, for fixed filter width $\Delta_{1}$, it found that the three-dimensional energy spectrum profile of Case 13 (grid $\delta x=0.125 \Delta_{1}$ and FGR 8) and Case 14 (grid $\delta x=0.0625 \Delta_{1}$ and FGR 16) are almost identical and appears that numerical solution converged at Case 13. Similarly, for fixed filter width $\Delta_{2}$ it appears that Case 23 (grid $\delta x=0.125 \Delta_{2}$ and FGR 8) and Case 24 (grid $\delta x=0.0625 \Delta_{2}$ and FGR 16) are almost collapse onto each other except in the high wavenumber region and the numerical convergence can be considered to be occurred at Case 23. For both filter width cases, it appears that relatively coarse grids (low FGRs) are unable to capture the energy containing eddies properly especially in Case 1. However increased resolution within the fixed filter width captures the energy containing eddies well. Moreover it resolves the small scale eddies at higher wavenumbers.

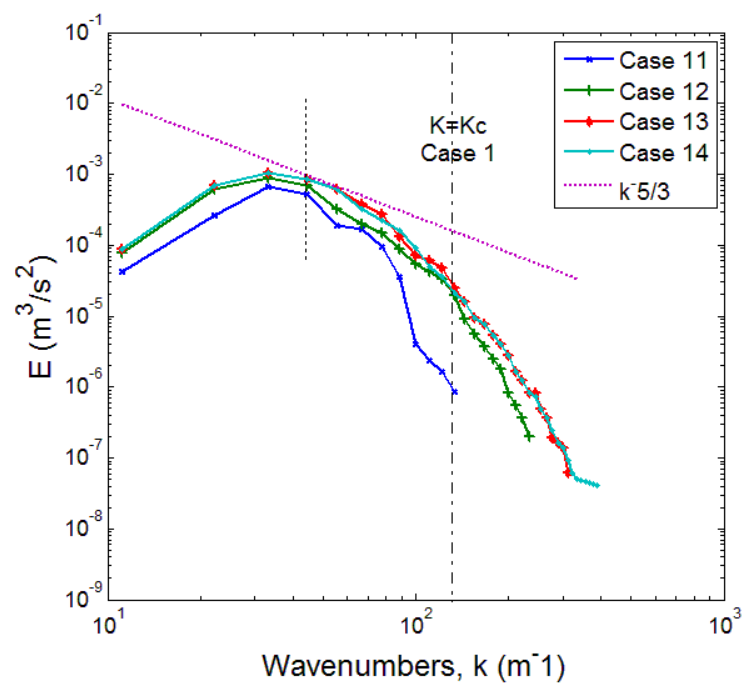

(a) Case $1\left(\Delta_{1}=0.2 \delta\right)$

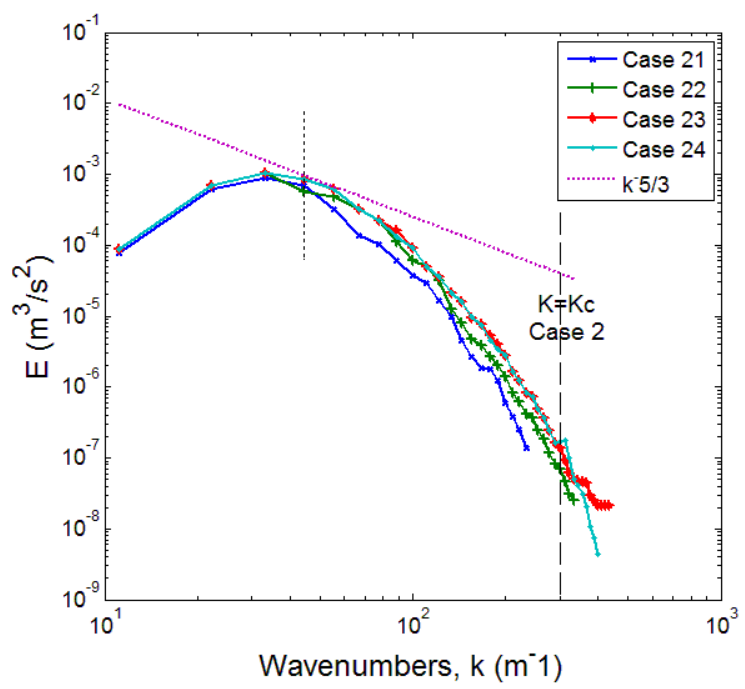

(b) Case $2\left(\Delta_{2}=0.1 \delta\right)$

Figure 5.3. Grid-convergence of three-dimensional energy spectra for the low Reynolds number flow with two different fixed filter widths 


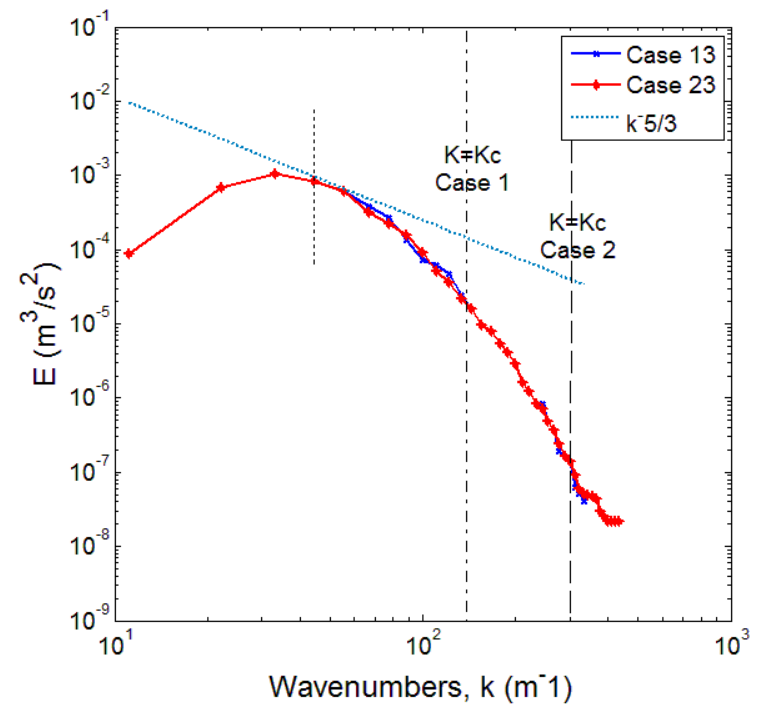

(a) Grid converged energy spectra of Case 1 $\left(\Delta_{1}=0.2 \delta\right)$ and Case $2\left(\Delta_{2}=0.1 \delta\right)$

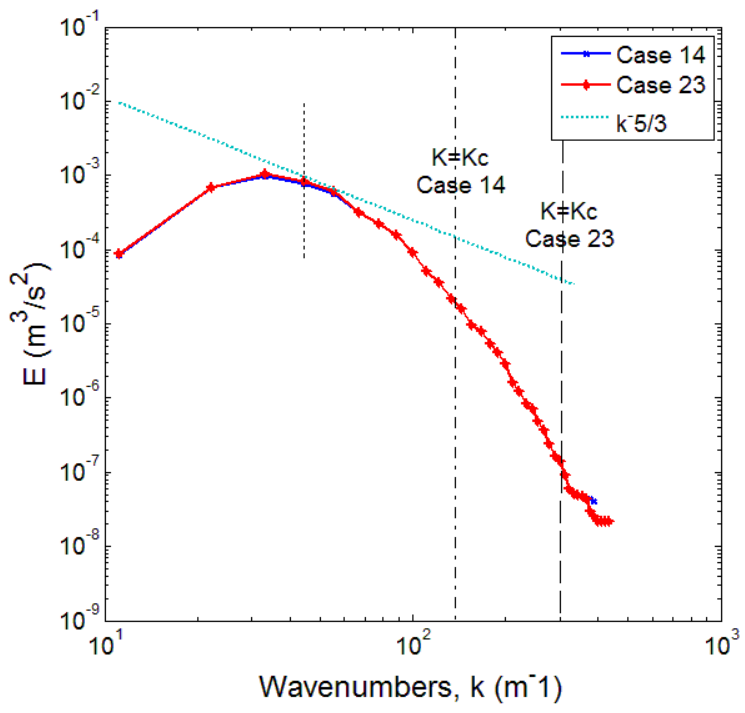

(b) Energy spectra with finest grids of Case 1 $\left(\Delta_{1}=0.2 \delta\right)$ and Case $2\left(\Delta_{2}=0.1 \delta\right)$

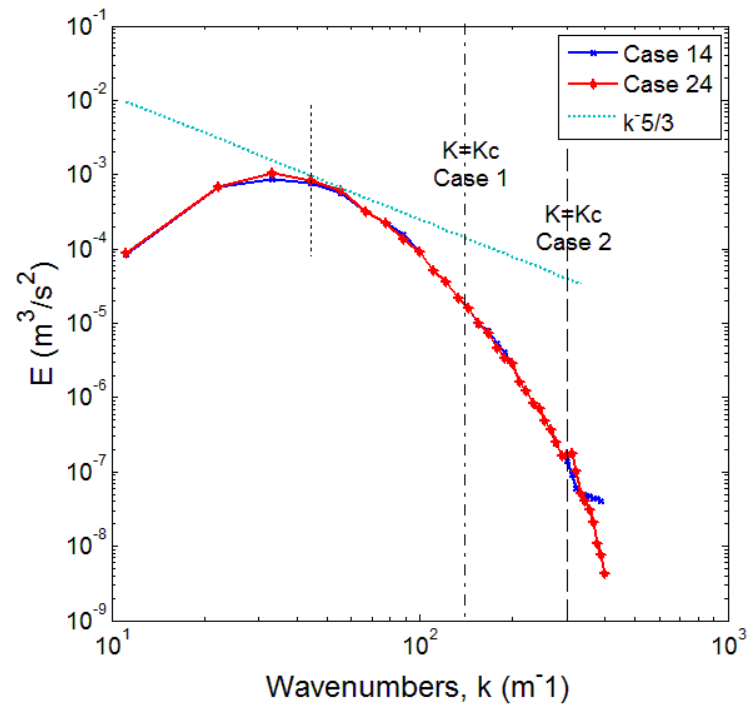

(c) Same grid resolution of Case $1\left(\Delta_{1}=0.2 \delta\right)$ and

Case $2\left(\Delta_{2}=0.1 \delta\right)$

Figure 5.4. Energy spectra analysis with various grid and fixed filter combination for Case 1

$$
\left(\Delta_{1}=0.2 \delta\right) \text { and Case } 2\left(\Delta_{2}=0.1 \delta\right)
$$

As for the high Reynolds number flow over the backward facing step, grid-convergence appears to occur when FGR is increased to 8 (i.e. Case 13 and Case 23 are both converged). The convergence is once again more rapid with increasing FGR when a finer filter is used. These numerical similarities between the grid-convergence of low and high Reynolds number energy 
spectra are overshadowed by the very obvious differences between the shapes of those spectra. While the energy containing range is well captured, it is quite apparent that the inertial sub-range does not approach the same theoretical scaling as does the high Reynolds number case, this phenomenon is also due to the fact that one flow is forced convection and the other one is natural convection. This makes it somewhat difficult to determine if the simulations conform to LES principles.

Grid converged spectra profiles of both filter width cases are compared in different combinations such as; (a) relatively coarse grids (Case 13 \& Case 23), (b) same grid size but different filter widths (Case $14 \&$ Case 23) and (c) finest grids (Case $14 \&$ Case 24). Comparison of grid converged solutions of energy spectra taken from figure 5.3 for filter width $\Delta_{1}$ and $\Delta_{2}$ are presented in figure 5.4(a). The comparison for finest grid resolutions Case 14 and Case 24 which are also grid converged solution of energy spectra for fixed filter widths $\Delta_{1}$ and $\Delta_{2}$ are presented in figure 5.4(b) but computationally 16 times expensive than Case 13 and Case 23. From the comparison, it is found that spectra profiles are following same pattern as in the earlier comparison. However, from the comparison it appears that a comparatively fine grid captures higher wavenumbers compared to relatively coarse grid while the filter width is the same and solution with both grids are free from numerical error. In a similar way, for same grid size but different filter widths, numerical solutions are compared between Case 14 and Case 23 is shown in figure 5.4(c). Comparison shows that relatively finer filter width $\Delta_{2}=0.1 \delta$ captures more wavenumbers along the $-5 / 3$ slope compared to the coarser filter width $\Delta_{1}=0.2 \delta$. It shows definite advantage of smaller filter width while the computational requirements will be the same. Like figure 5.3, the theoretical $-5 / 3$ slope is plotted in the figure 5.4 to identify the inertial sub range and a small vertical demarcation to separate energy containing and inertial range. It is seen that the inertial range is very small in both cases (presented in figure 5.3 and figure 5.4). It is assumed that in energy spectra profiles, the reason that the inertial slope is not developing 
properly is due to low Reynolds number. Several authors (Mansour et al. 1994, Sreenivasan 1994 and Djenidi et al. 2013) suggest that inertial range in low Reynolds number $\left(R e_{\lambda}<50\right)$ is scarcely manifest in the energy spectra because the lengthscale separation among the large scale and small scale eddies is too small.

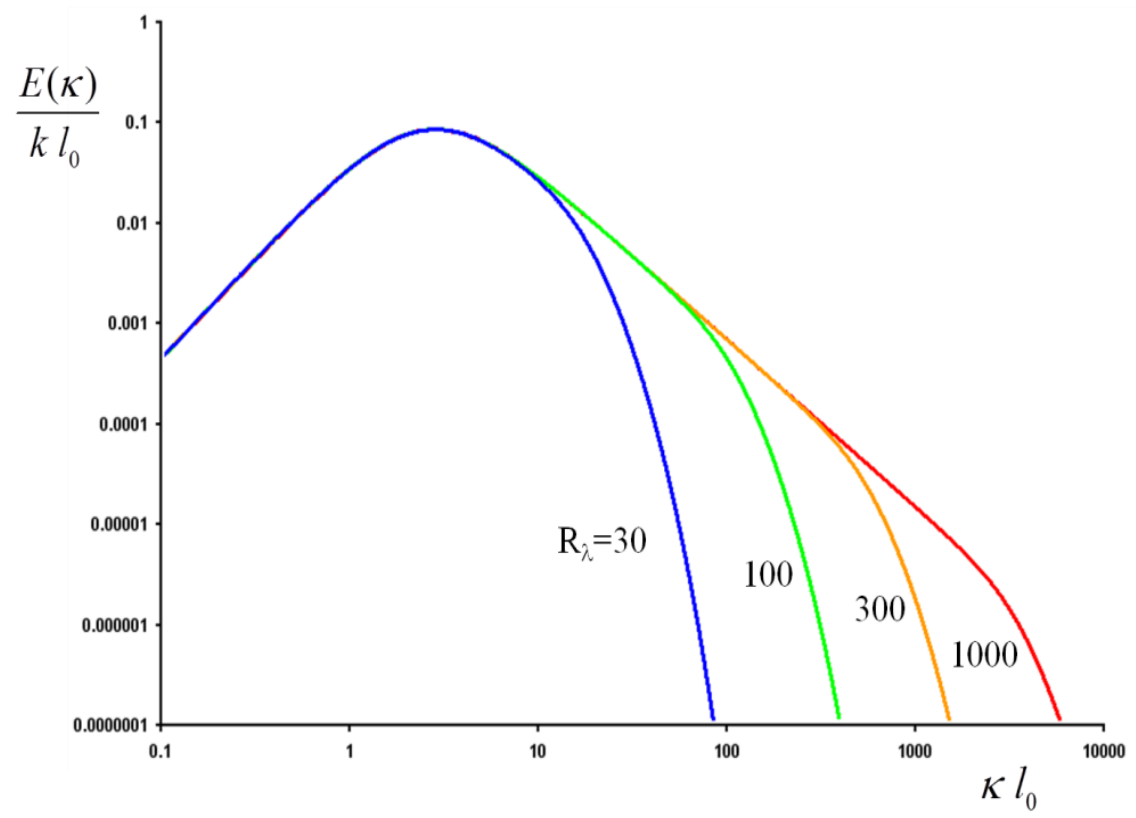

Figure 5.5. The model spectra at various Reynolds numbers (Pope 2000)

To justify this above mentioned fact, Pope (2000) suggests the model energy spectrum at different Reynolds numbers as shown in figure 5.5 where values of spectra $E(\kappa)$ and wavenumbers $\kappa$ are normalized by the integral lengthscale $(l)$ and turbulent kinetic energy $(k)$ respectively and presented as universal form. The expression for model spectra function is,

$$
E(\kappa)=C \varepsilon^{2 / 3} \kappa^{-5 / 3} f_{l}(\kappa l) f_{\eta}(\kappa \eta)
$$

For large $\kappa l$, the function of wavenumbers based on integral lengthscale $f_{l}(\kappa l)$ will become unity in the above expression. Here, $C$ is the Kolmogorov constant and $f_{\eta}(\kappa \eta)$ is function of wavenumbers normalized by the Kolmogorov lengthscale $(\eta)$ can expressed as,

$$
f_{\eta}(\kappa \eta)=\exp \left[-\beta\left(\left[(\kappa \eta)^{4}+c_{\eta}^{4}\right]^{1 / 4}-c_{\eta}\right)\right]
$$

where, $\beta$ and $c_{\eta}$ are constants. 
It clearly appears from the figure 5.5 that at low Reynolds number energy spectra do not necessarily follow the Kolmogorov universal scaling in the inertial subrange and the range is very small so that it hardly exists in the spectra. It also shows that the theoretical universal slope $-5 / 3$ which is proportional to the wavenumbers $\kappa^{-5 / 3}$ develops at relatively high Reynolds numbers where the energy containing range (eddies are more anisotropic in nature) and universal equilibrium range (eddies are isotropic in nature) exist clearly. This inertial range can be expressed as a constant slope on the spectrum.

If we again examine figure 5.4 it appears that the results of combinations of grid-converged solutions of energy spectra of selected filter widths of cavity flow are clearly in agreement and following the same trend with the model spectra for low Reynolds number flows. We could say that it may have a very small $-5 / 3$ slope region that would just be a tangent to the profile of spectra which separates the energy containing and inertial subrange that has been presented by a small demarcation line in both Figures 5.3 and 5.4. From the figures it appears that the cut off wavenumbers $\left(\kappa_{c}\right)$ corresponding to the selected filter widths taken as $20 \%$ and $10 \%$ of the BLT are within the inertial subrange and are capturing the energy containing range very well, which also satisfies the principle of LES. From Reynolds number dependent model spectra such as those shown in Figure 5.5 it is found that the spectrum corresponding to a Taylor Reynolds number of 30 is very similar in shape to that obtained here for $R e_{\lambda}$. A similar configuration studied by Lau et al. (2013) also produced similar spectra to those shown below. Although analytically difficult to determine convergence to LES principles at such low Reynolds numbers the supporting literature gives us reasonable confidence that our simulations are in fact LES compliant. Therefore no simulation is conducted taking 5\% of BLT as the filter width. 


\subsubsection{The congruence of energy spectra results with flow variables}

The accurate modelling of energy spectra is congruent with the accuracy of two dimensionless flow variables, namely mean velocity and non-dimensional mean temperature is presented in this section. Both of the variables are equally important for describing the nature of the flow qualitatively and quantitatively inside the cavity.

\section{Mean velocity}

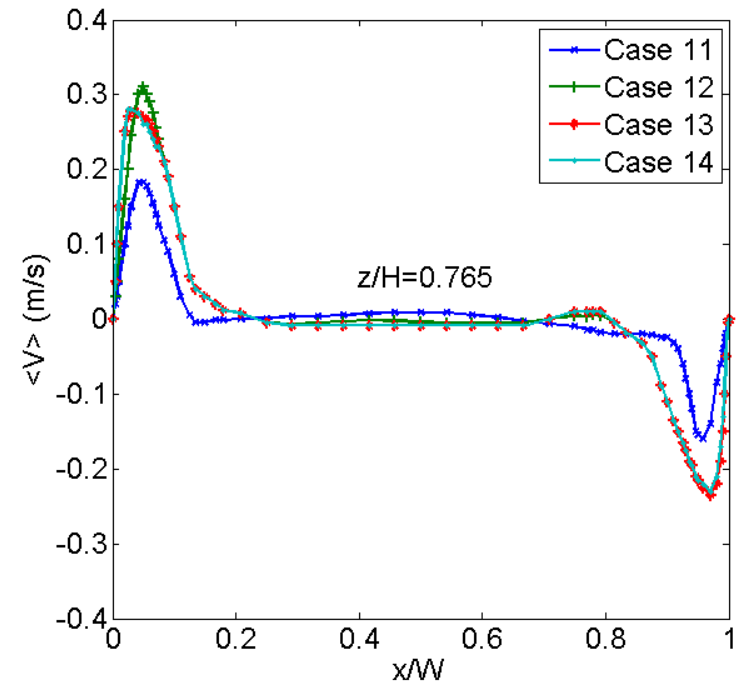

(a) At test section $z / H=0.765$

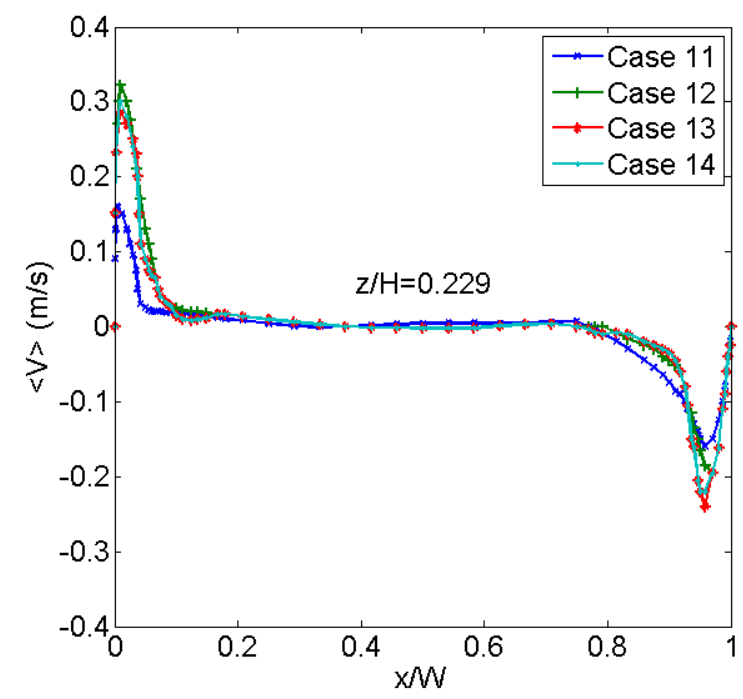

(c) At test section $z / H=0.229$

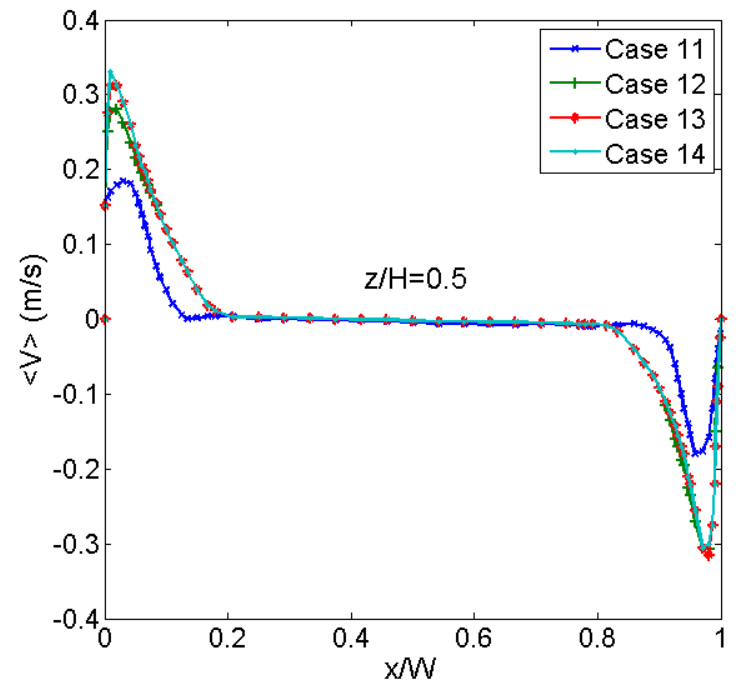

(b) At test section $z / H=0.5$

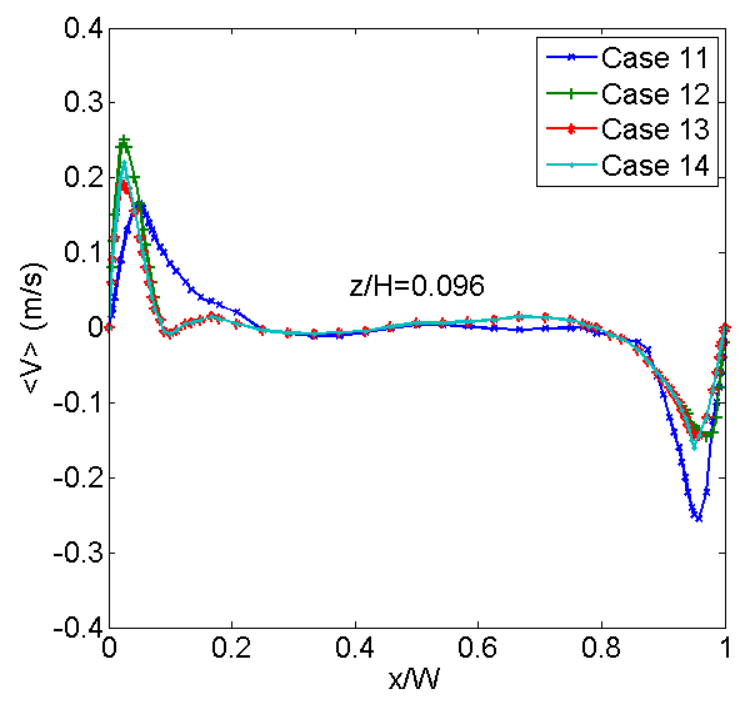

(d) At test section $z / H=0.096$

Figure 5.6. Mean velocity at four test sections for fixed filter Case $1\left(\Delta_{1}=0.2 \delta\right)$ 
Figure 5.6 presents the mean velocity, obtained from Cases 11-14 for fixed filter width Case 1 $\left(\Delta_{1}=0.2 \delta\right)$, at four locations along the cavity height. From Figure 5.6, it appears that mean velocity profiles at different test locations in Case $13\left(\delta x=0.125 \Delta_{1}\right.$ and FGR 8) and Case 14 $\left(\delta x=0.0625 \Delta_{1}\right.$ and FGR 16) are closely matched to each other, similar to the energy spectra in Figure 5.3(a). In both cases, the estimated $n_{\min }^{+}$value is approximately $0.65-1.15$. These are also closely followed by Case 12 profiles. Therefore, mean velocity profiles demonstrate the numerically converged solution at grid resolutions of Case 13.

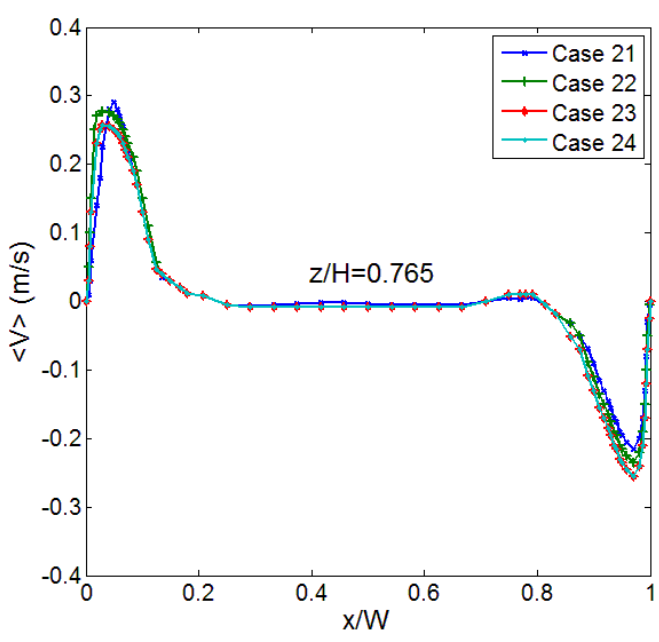

(a) At test section $z / H=0.765$

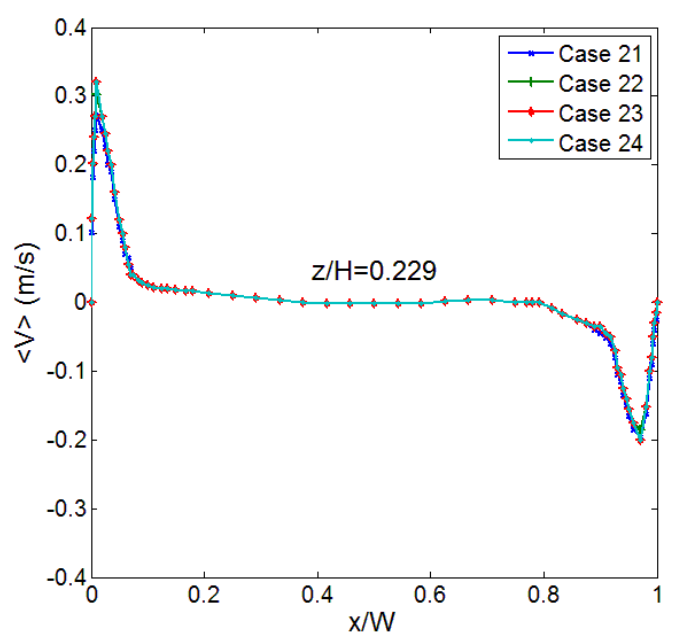

(c) At test section $z / H=0.229$

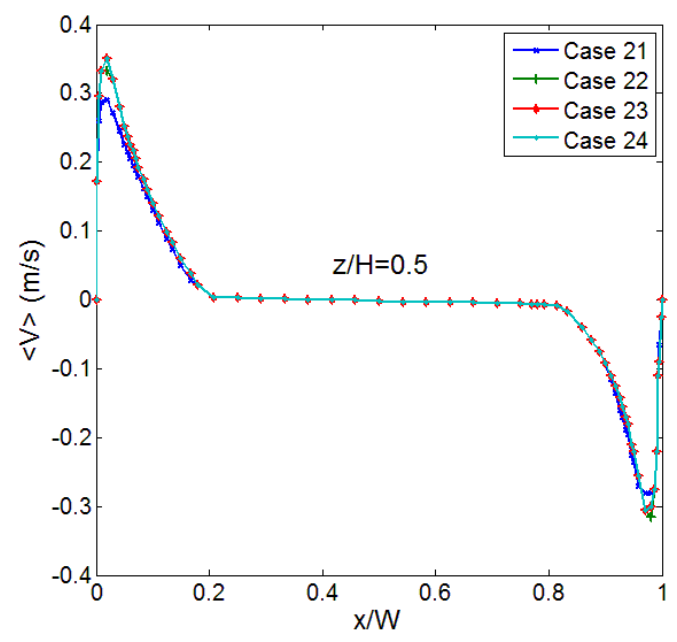

(b) At test section $z / H=0.05$

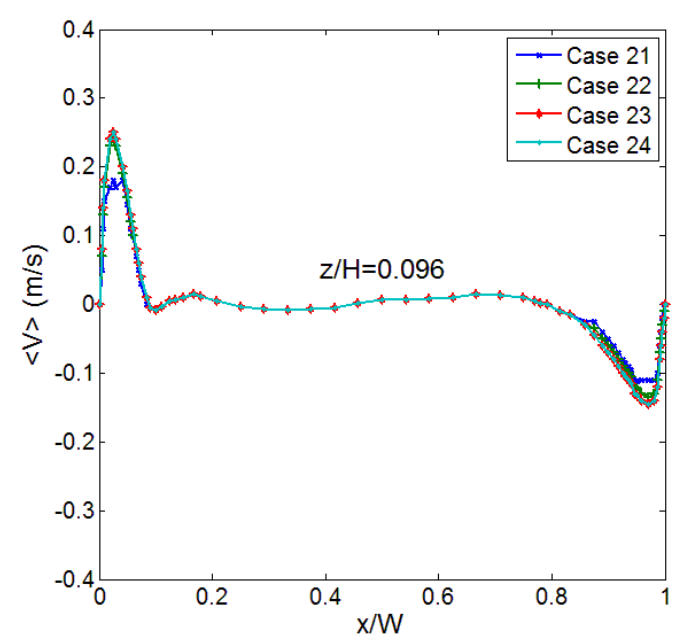

(d) At test section $z / H=0.096$

Figure 5.7. Mean velocity at four test sections for fixed filter Case $2\left(\Delta_{2}=0.1 \delta\right)$ 
Figure 5.7 shows mean velocity profiles obtained from Cases 21-24 (where $\Delta_{2}=0.1 \delta$ ) at four locations. It can be observed that Case $23\left(\delta x=0.125 \Delta_{2}\right.$ and FGR 8) and Case $24\left(\delta x=0.0625 \Delta_{2}\right.$ and FGR 16) completely collapse onto each other and furthermore the Case 22 solutions are also very close to these solutions. This reflects Figure 5.3 (b) and $n_{\min }^{+}$value for these cases are 0.65 or below.

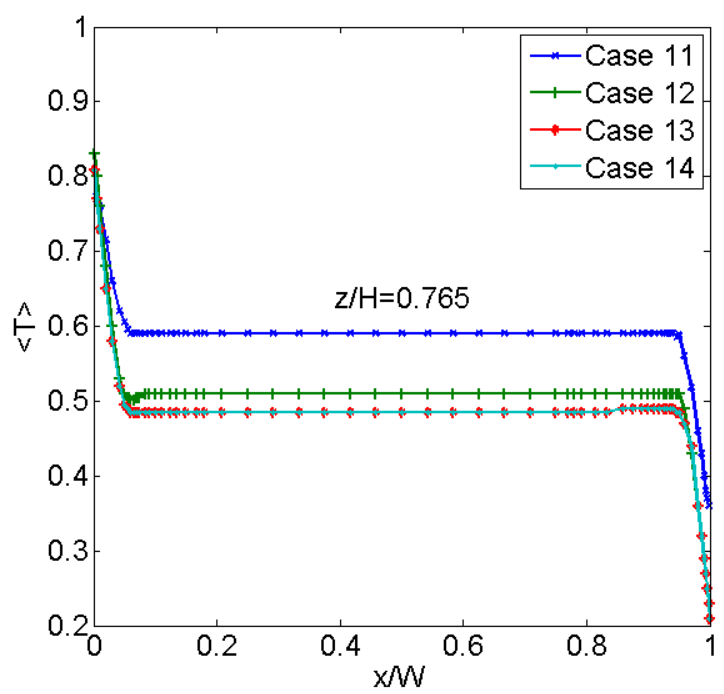

(a) At test section $z / H=0.765$

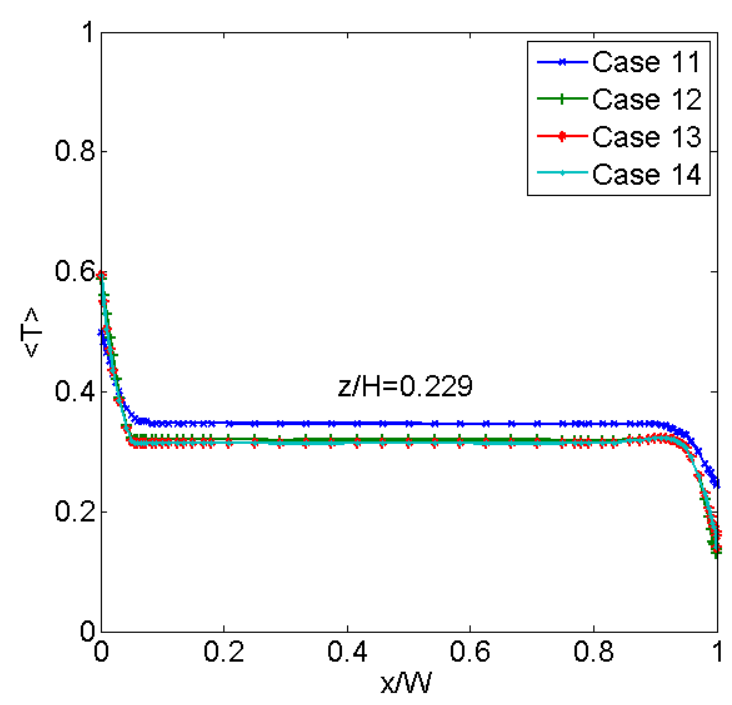

(c) At test section $z / H=0.229$

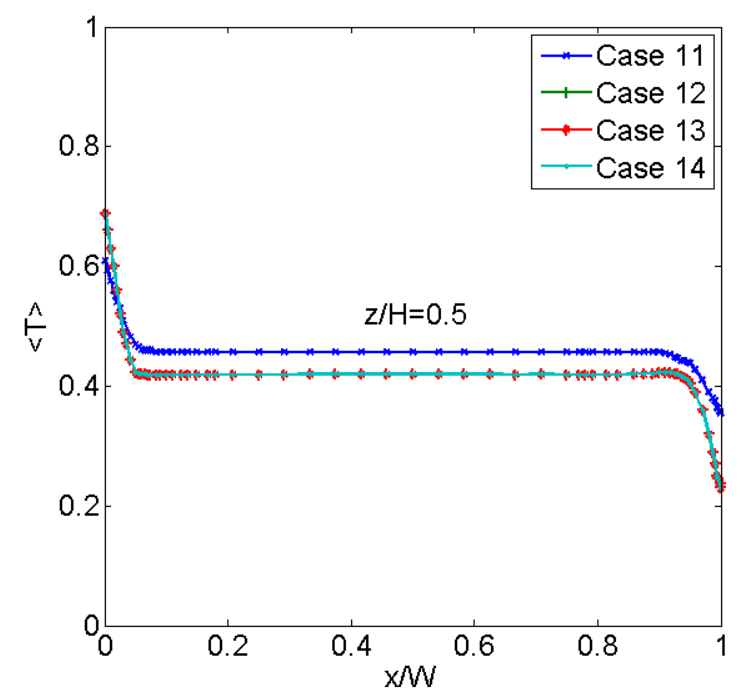

(b) At test section $z / H=0.5$

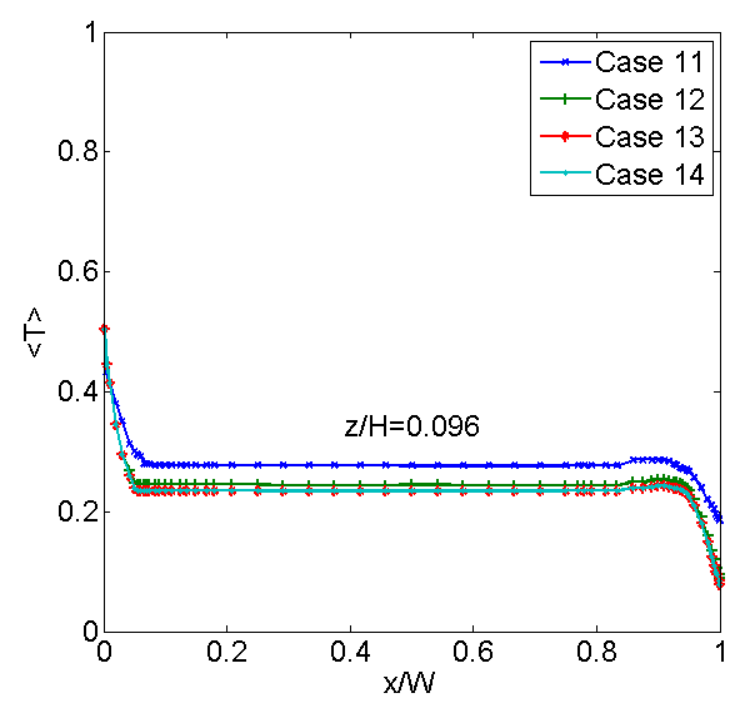

(d) At test section $z / H=0.096$

Figure 5.8. Mean temperatures at four test sections for fixed filter Case $1\left(\Delta_{1}=0.2 \delta\right)$ 


\section{Non dimensional mean temperature}

In Figures 5.8 and 5.9, the non-dimensional mean temperature inside the cavity have been presented which is calculated by using the following equation,

$$
<T>=\left(\tilde{T}-T_{\text {cold }}\right) /\left(T_{\text {hot }}-T_{\text {cold }}\right)
$$

where, $\tilde{T}$ is the Favre averaged temperature of fluid and the temperature of cold and hot walls are presented by $T_{\text {cold }}$ and $T_{\text {hot }}$ respectively.

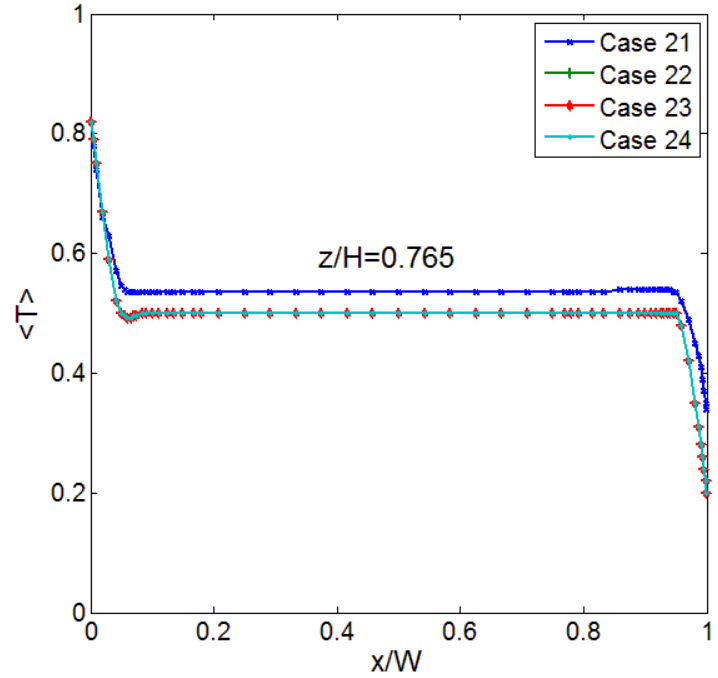

(a) At test section $z / H=0.765$

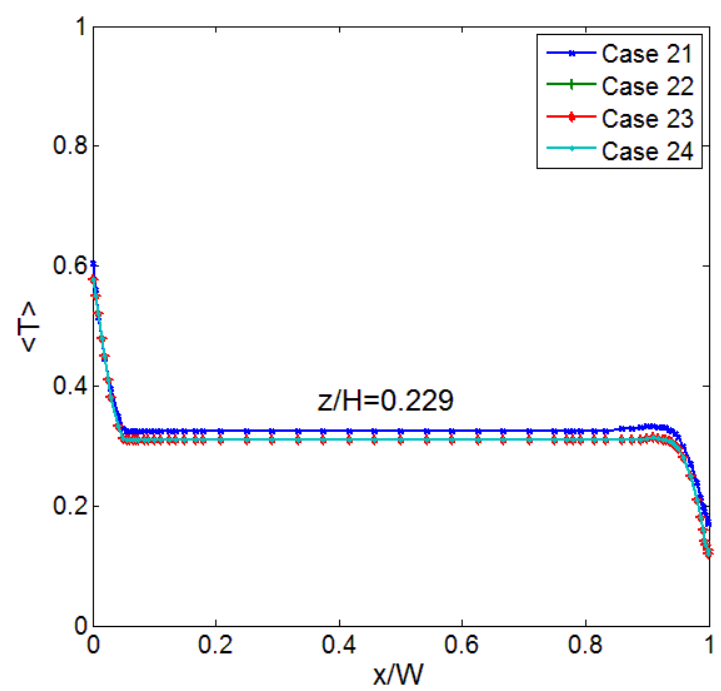

(c) At test section $z / H=0.229$

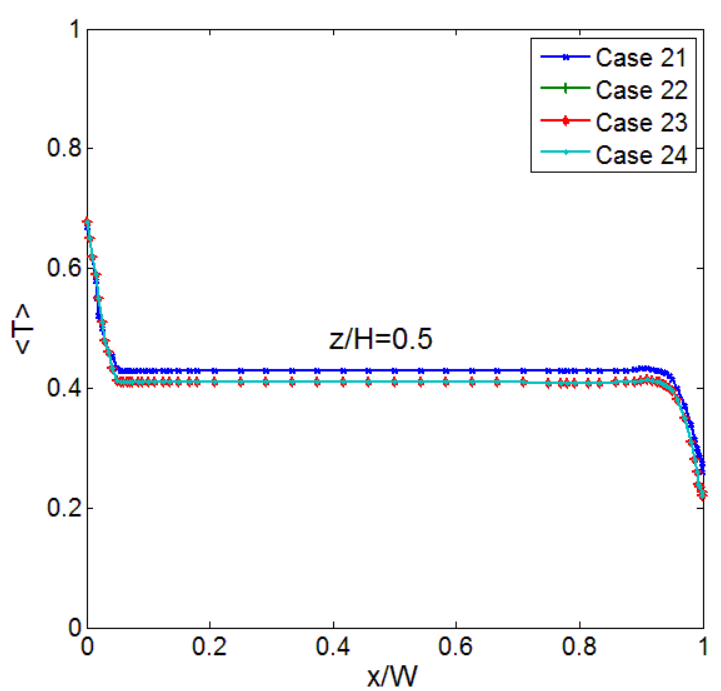

(b) At test section $z / H=0.5$

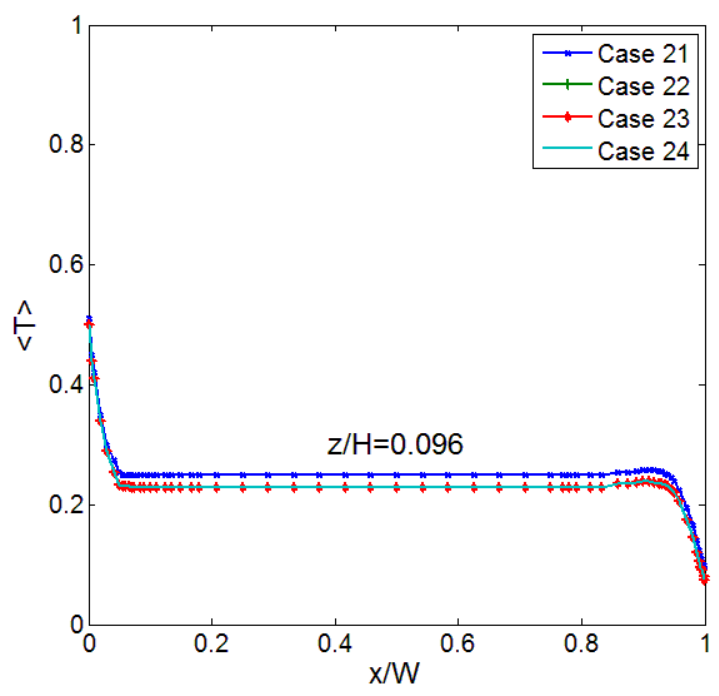

(d) At test section $z / H=0.096$

Figure 5.9. Mean temperature at four test sections for fixed filter Case $2\left(\Delta_{2}=0.1 \delta\right)$ 
Similar to the mean velocity profile, reflection of energy spectra on non-dimensional mean temperature has been presented in Figures 5.8 and 5.9 for filter width Case $1\left(\Delta_{1}=0.2 \delta\right)$ and Case $2\left(\Delta_{2}=0.1 \delta\right)$ at the same test locations inside the cavity. It is to be noted that data right on the walls have not been included, therefore although according to definition $(5.5)<\mathrm{T}\rangle$ should be equal to 1 at the hot wall and zero at the cold wall, these are not presented. From Figure 5.8, for Case $1\left(\Delta_{1}=0.2 \delta\right)$, it appears that the numerical solution obtained for Case 13 (grid $\delta \mathrm{x}=0.125 \Delta_{1}$ and FGR 8) and Case 14 (grid $\delta x=0.0625 \Delta_{1}$ and FGR 16) completely match each other. This is consistent with Figures 5.3 (a) and 5.6. This is evident from Figure 5.3 that larger filter width with low FGR is not capable to capture all energy containing eddies. This effects other flow variables therefore mean temperatures obtained from Case $11\left(\delta \mathrm{x}=0.5 \Delta_{1}\right.$ and FGR 2$)$ and Case $12\left(\delta \mathrm{x}=0.25 \Delta_{1}\right.$ and FGR 4$)$ are significantly different from the other two cases.

On the other hand, Figure 5.9 shows reflection of energy spectra on mean temperature for fixed filter width Case $2\left(\Delta_{2}=0.1 \delta\right)$. It can be observed that dimensionless mean temperature profiles obtained from Case $23\left(\delta \mathrm{x}=0.125 \Delta_{2}\right.$ and FGR 8$)$ and Case $24\left(\delta \mathrm{x}=0.0625 \Delta_{2}\right.$ and FGR 16) completely collapse onto each other. It also appears that the Case $22\left(\delta \mathrm{x}=0.25 \Delta_{2}\right.$ and FGR 4$)$ solution shows very marginal difference compared to the converged solutions. This is consistent with Figures 5.3 (b) and 5.7.

\subsubsection{Validation of the proposed model}

For each filter width case, first, the simulations results with two finest resolutions are compared graphically. Then the results are compared with the experimental results of Cheesewright et al. (1986) using statistical relative error analysis. For the latter, the method of Ierardi et al. (2003) has been followed which is described in Chapter 3 . 


\subsubsection{Graphical comparison}

Grid-converged solutions of Case 13 and Case 23 are considered as the most accurate of the fixed filter widths $\Delta_{1}$ and $\Delta_{2}$ respectively, graphical comparison of mean velocity and dimensionless mean temperature is made for these two cases first. Afterwards, the combinations of Case 14 and Case 24 are considered as the finest grid resolutions of each of the filter width cases, and Cases 14 and 23 as the same grid resolutions but different filter width has been discussed for validations of results of both flow variables.

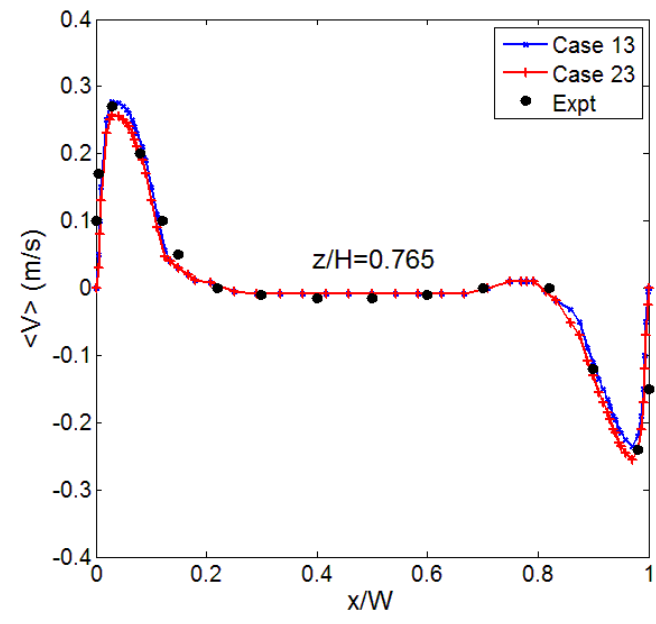

(a) At test section $z / H=0.765$

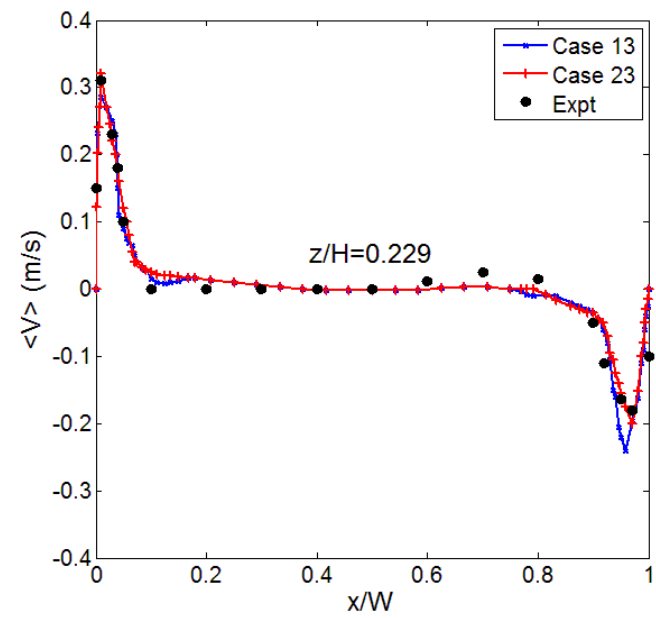

(c) At test section $z / H=0.229$

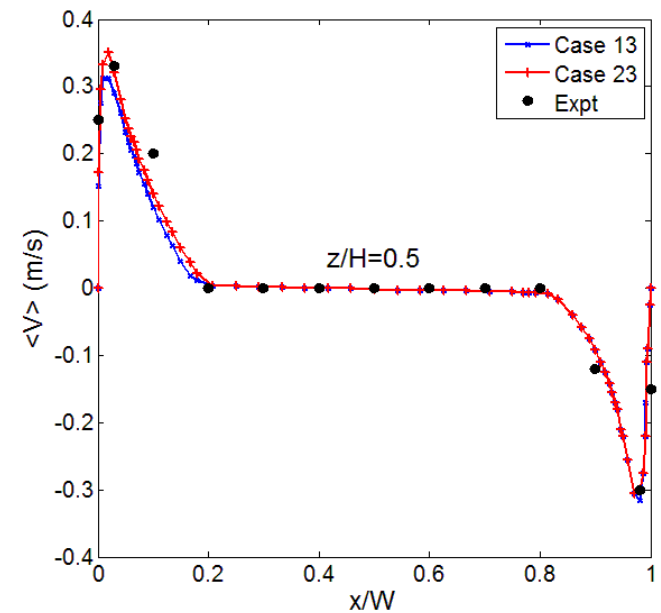

(b) At test section $z / H=0.5$

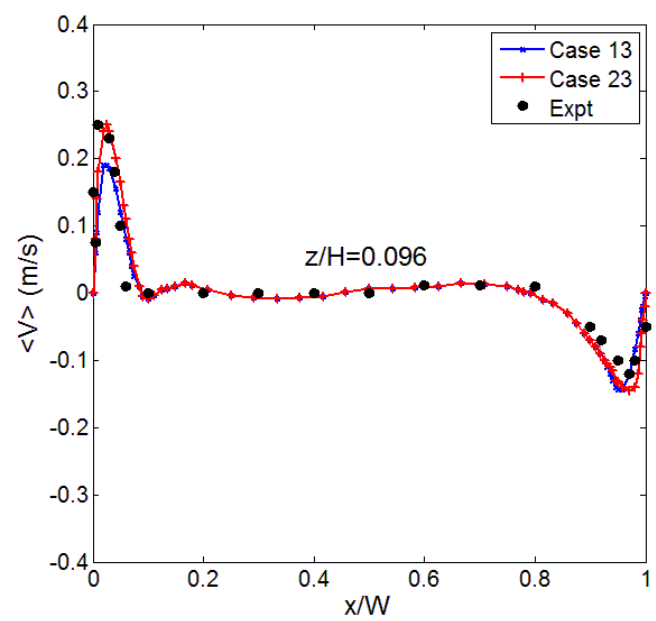

(d) At test section $z / H=0.096$

Figure 5.10. Mean velocity grid converged solution at relatively coarser grid resolution for fixed filter Case $1\left(\Delta_{1}=0.2 \delta\right)$ and Case $2\left(\Delta_{2}=0.1 \delta\right)$ 


\section{Mean velocity}

\section{Comparison of coarser grid resolutions}

Figure 5.10, portrays a comparison of the mean velocity profiles of Case $13\left(\delta x=0.125 \Delta_{1}\right.$ and FGR 8) and Case $23\left(\delta x=0.125 \Delta_{2}\right.$ and FGR 8) with the experimental data of Cheesewright et al. (1986). It can be seen that the predicted values are in overall good agreement with the experiments. Both simulations correctly capture the trend for increasing buoyant velocity and corresponding boundary layer thickening with height in the cavity. Case 23 with the finer filter width predicts the magnitude of the peak velocities a little better than does simulate Case 13 .

\section{Comparisons at the finest grid resolutions}

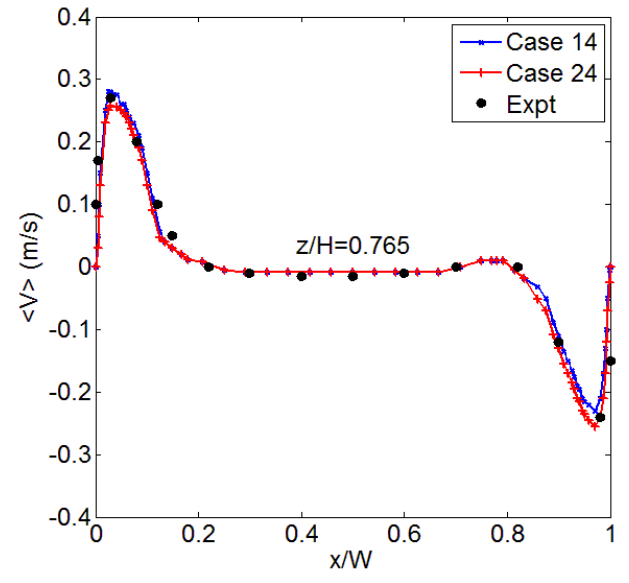

(a) At test section $z / H=0.765$

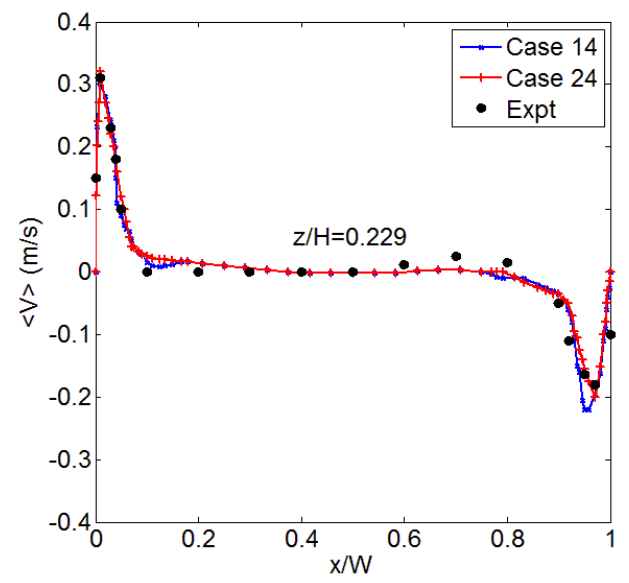

(c) At test section $z / H=0.229$

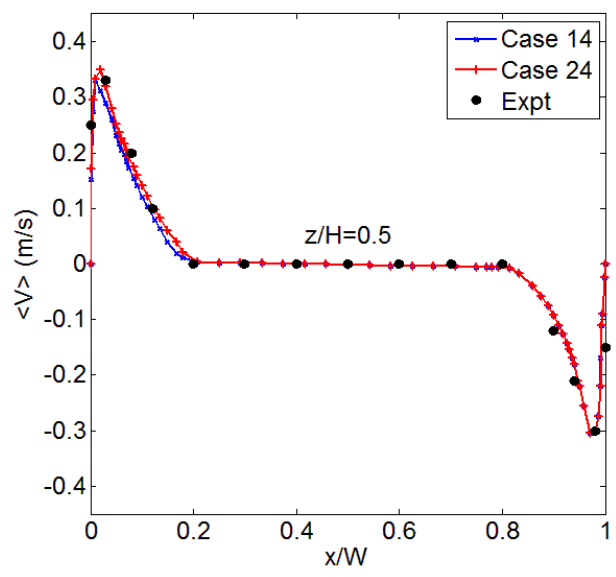

(b) At test section $z / H=0.5$

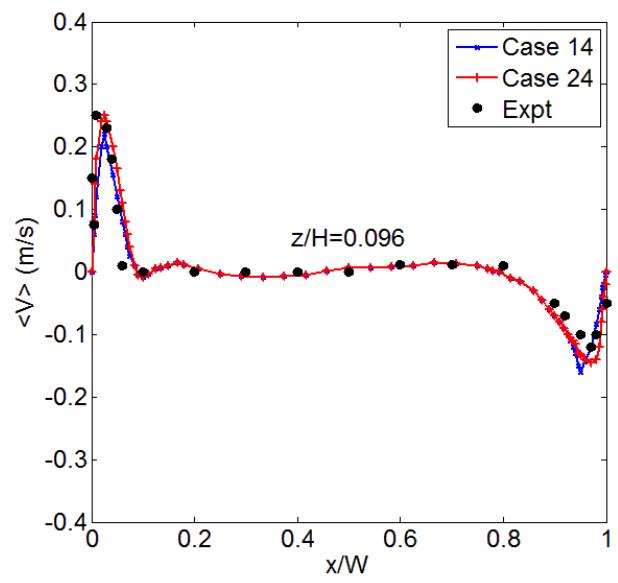

(d) At test section $z / H=0.096$

Figure 5.11. Mean velocity obtained with the finest grid resolution for fixed filter Case 1

$$
\left(\Delta_{1}=0.2 \delta\right) \text { and Case } 2\left(\Delta_{2}=0.1 \delta\right)
$$


Figure 5.11 shows a comparison of the results of grid-converged solutions with the finest grid resolution of each of the fixed filter width cases. For Case 1 and Case 2, these are Case 14 $\left(\delta \mathrm{x}=0.0625 \Delta_{1}\right.$ and FGR 16) and Case $24\left(\delta \mathrm{x}=0.0625 \Delta_{2}\right.$ and FGR 16) respectively. From a comparison of the results, it appears that Case 24 shows better agreement to the existing experimental data compared to Case 14 at all selected test locations inside the differentially heated cavity.

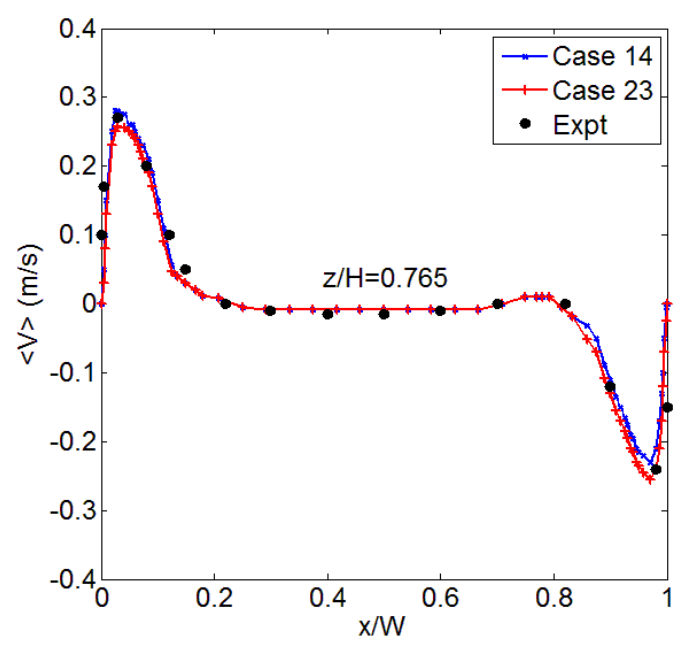

(a) At test section $z / H=0.765$

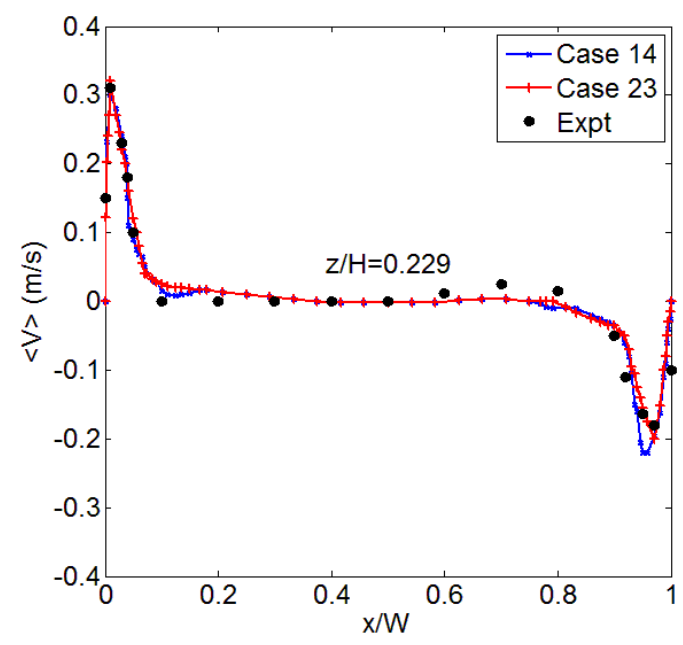

(c) At test section $z / H=0.229$

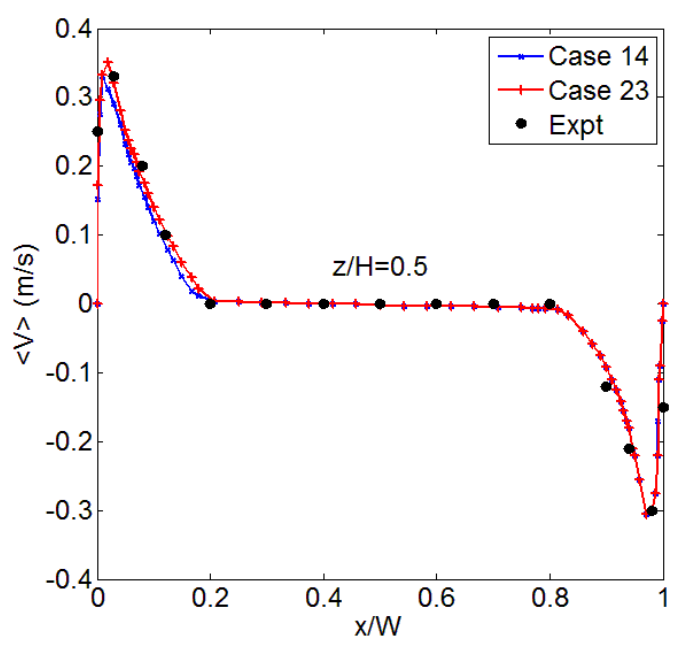

(b) At test section $z / H=0.5$

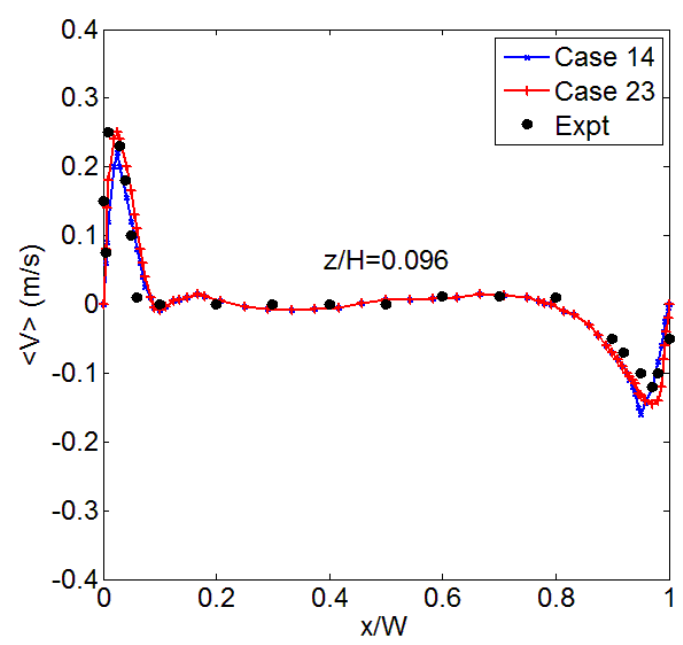

(d) At test section $z / H=0.096$

Figure 5.12. Mean velocity grid converged solution at same grid resolution for fixed filter Case 1

$$
\left(\Delta_{1}=0.2 \delta\right) \text { and Case } 2\left(\Delta_{2}=0.1 \delta\right)
$$




\section{The effect of filter width}

Figure 5.12 shows a comparison of the grid-converged solution of fixed filter Case 1 and Case 2 for the same grid resolutions at different filter width. Presented in Table 5.2, the same grid resolutions at different filter width and filter to grid spacing ratios (FGR), are Case 14 $\left(\delta x=0.0625 \Delta_{1}\right.$ and FGR 16) and Case $23\left(\delta x=0.125 \Delta_{2}\right.$ and FGR 8).

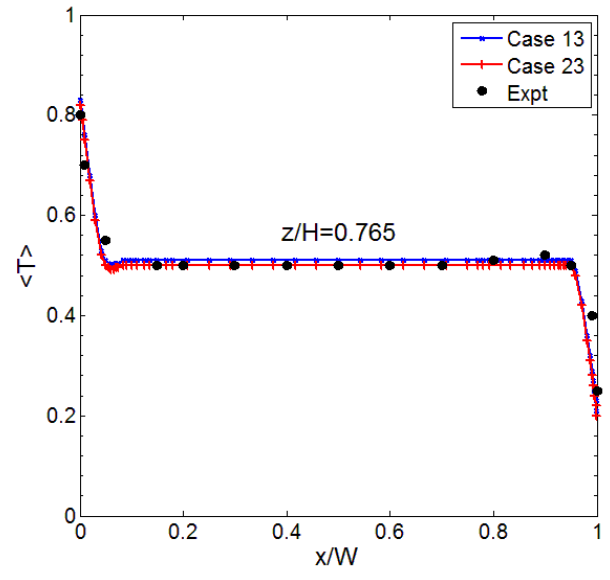

(a) At test section $z / H=0.765$

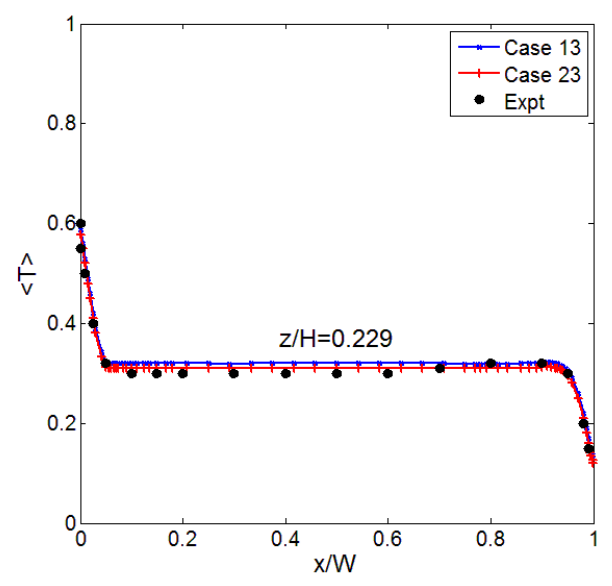

(c) At test section $z / H=0.229$

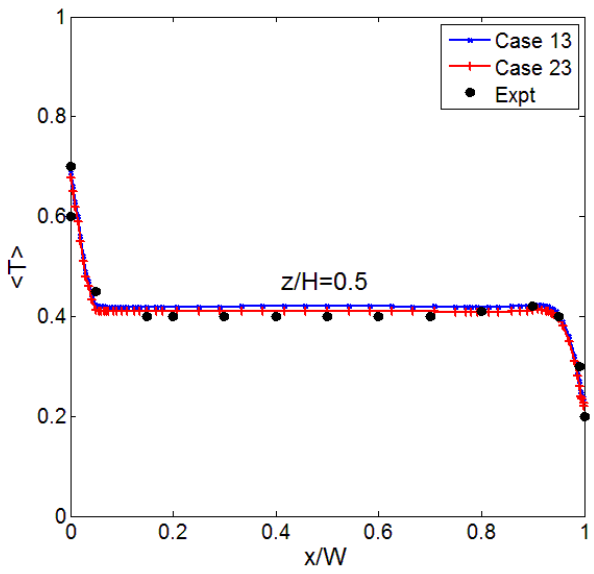

(b) At test section $z / H=0.5$

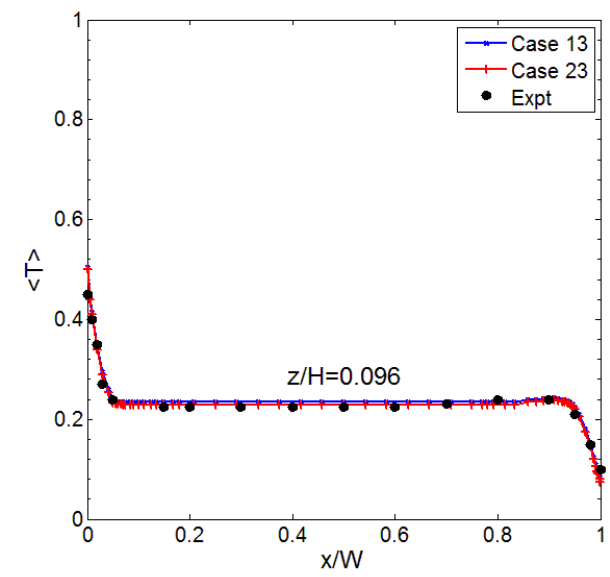

(d) At test section $z / H=0.096$

Figure 5.13. Mean temperature grid-converged solutions at relatively coarser grid resolutions for fixed filter Case $1\left(\Delta_{1}=0.2 \delta\right)$ and Case $2\left(\Delta_{2}=0.1 \delta\right)$

From the comparisons, it is found that Case 23 shows relatively better agreement with the experimental results compared to Case 14 at selected test sections. Moreover, it appears that for the same grid resolutions at different filter widths, relatively a finer filter width is likely to provide more accurate numerical solutions. 
Overall it can be observed that findings of figures 5.10, 5.11 and 5.12 are consistent with the findings of figures 5.4(a), 5.4 (b) and 5.4(c), respectively.

\section{Non dimensional mean temperature}

\section{Comparison of coarser grid resolutions}

Figure 5.13 shows comparisons of the mean dimensionless temperature at the same test locations and also for Case 13 and Case 23. Here both simulations capture the thermal boundary layer accurately while the finer filter case produces slightly more accurate results across the middle of the cavity where uniform temperature distributions are found.

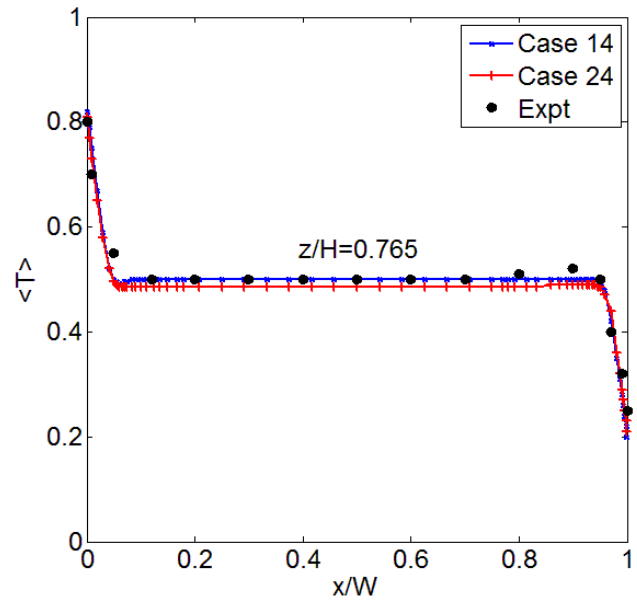

(a) At test section $z / H=0.765$

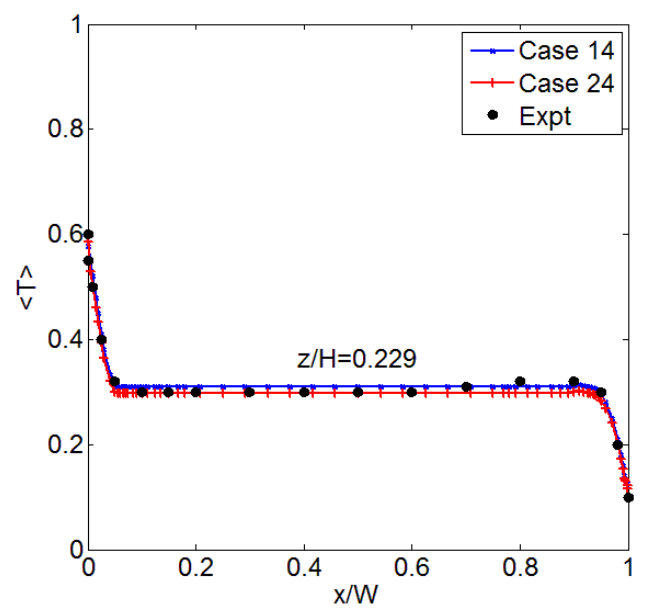

(c) At test section $z / H=0.229$

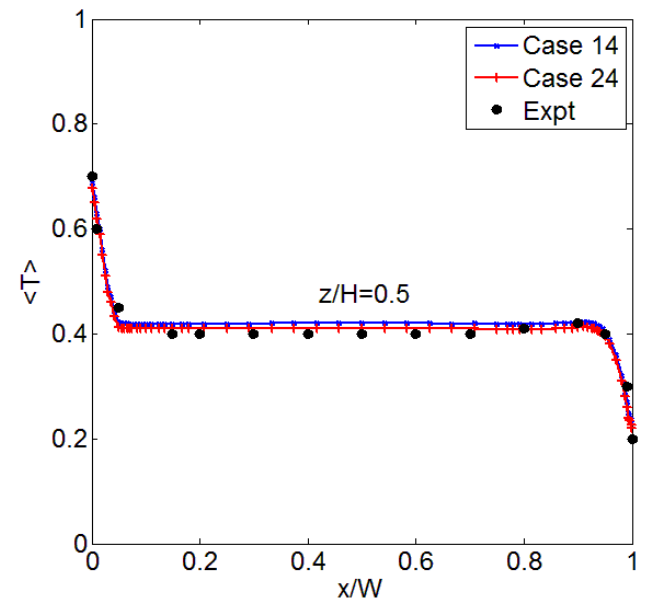

(b) At test section $z / H=0.5$

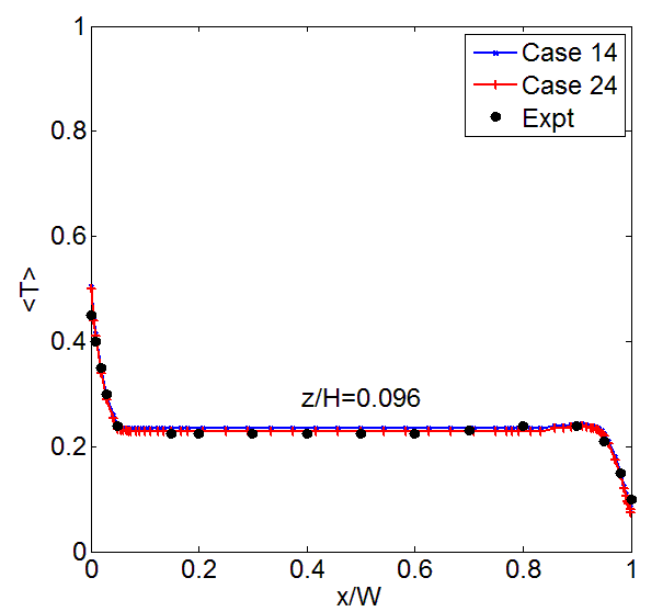

(d) At test section $z / H=0.096$

Figure 5.14. Mean temperature obtained with the finest grid resolution for fixed filter Case 1

$$
\left(\Delta_{1}=0.2 \delta\right) \text { and Case } 2\left(\Delta_{2}=0.1 \delta\right)
$$




\section{Comparisons at the finest grid resolutions}

Figure 5.14 shows the results for comparison of grid-converged solutions with the finest grid resolution of each fixed filter width cases. For, Case 1 and Case 2, these are Case 14 $\left(\delta \mathrm{x}=0.0625 \Delta_{1}\right.$ and FGR 16) and Case $24\left(\delta \mathrm{x}=0.0625 \Delta_{2}\right.$ and FGR 16) respectively. From a comparison of the results, it appears that Case 24 shows relatively better agreement to the existing experimental data compared to Case 14 at selected test locations except at $z / H=0.765$ inside the cavity.

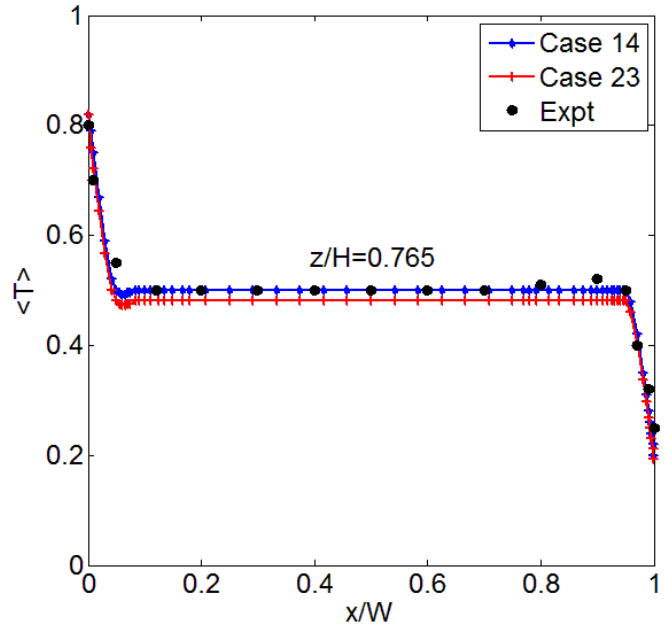

(a) At test section $z / H=0.765$

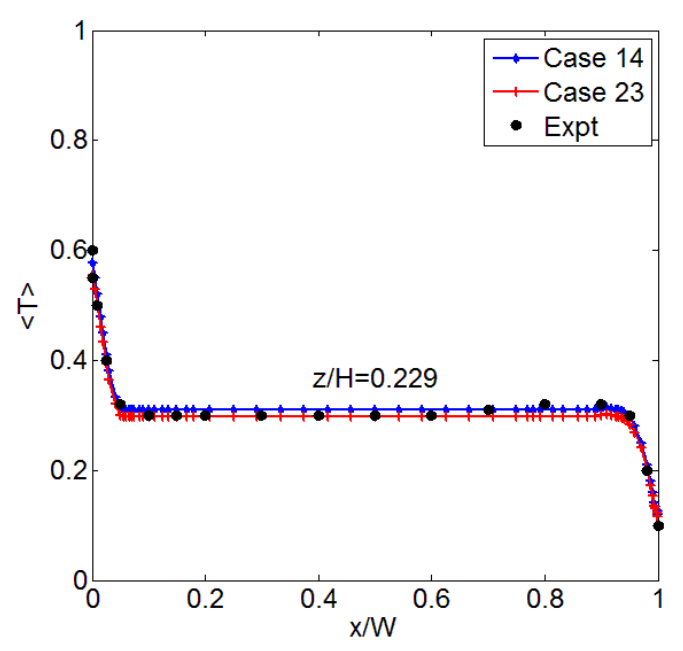

(c) At test section $\mathrm{z} / \mathrm{H}=0.229$

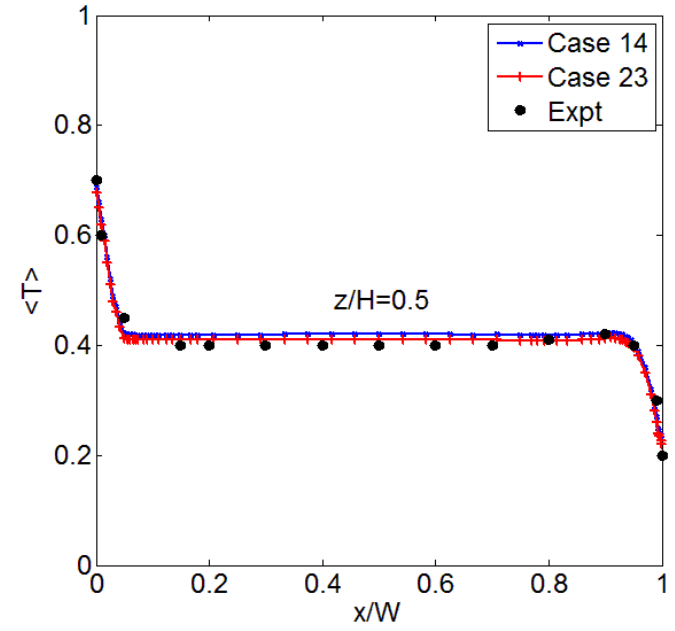

(b) At test section $z / H=0.5$

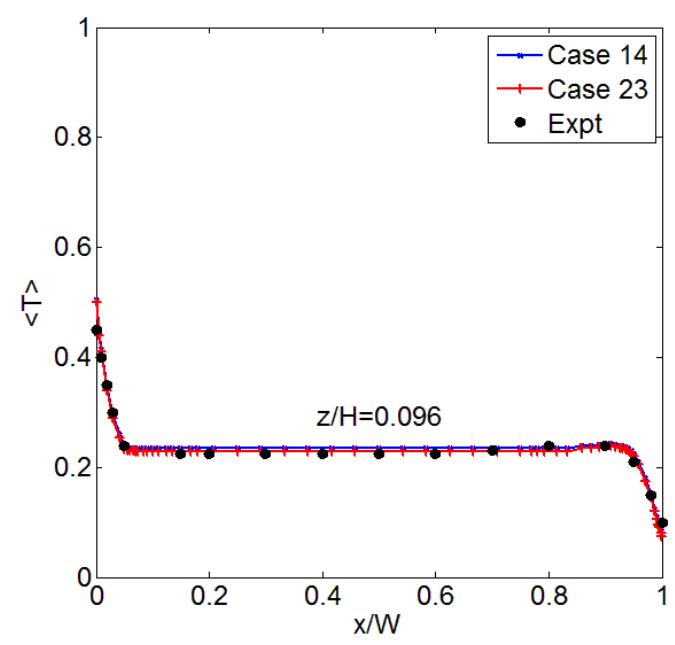

(d) At test section $\mathrm{z} / \mathrm{H}=0.096$

Figure 5.15. Mean temperature grid converged solution at same grid resolution for fixed filter Case $1\left(\Delta_{1}=0.2 \delta\right)$ and Case $2\left(\Delta_{2}=0.1 \delta\right)$ 


\section{The effect of filter width}

Figure 5.15 shows a comparison of the grid-converged solution of fixed filter Case 1 and Case 2 for the same grid resolutions but different filter widths. The same grid resolutions at different filter widths and filter to grid spacing ratios (FGR) are Case $14\left(\delta x=0.0625 \Delta_{1}\right.$ and FGR 16) and Case $23\left(\delta x=0.125 \Delta_{2}\right.$ and FGR 8$)$. From the comparison, it is found that Case 23 shows slightly better agreement to the experimental results compared to Case 14 at selected test sections except at $z / H=0.765$. Moreover, similarly to the mean velocity profiles, it appears that for mean temperature for the same grid resolutions at different filter widths, relatively finer filter width is likely to provide more accurate numerical solutions.

Overall, it can be observed that findings of figures 5.13-15 are consistent with the findings of figure 5.4 .

\section{Summary of results validation}

For buoyancy driven cavity flow overall grid-converged solutions with filter width $\Delta_{2}$ taken as $10 \%$ of the BLT, show comparatively better agreement over filter width $\Delta_{1}$ taken as $20 \%$ of BLT. It also appears that Case 23 with filter width $\Delta_{2} 10 \%$ of BLT and FGR 8 shows very good agreement to the existing reference data for flow variables such as mean velocity and mean temperature. From a computational resource point of view, it requires eight times fewer grid cells than Case 24 apart from having reduced temporal resolution need. More details about the computational cost is discussed in section 5.2.3.2.

\subsubsection{Statistical analysis of predicted outcomes}

Tables 5.3 and 5.4 show the cross-stream averaged relative errors of mean velocity and mean dimensionless temperature, respectively. The results are for all grid-converged cases (i.e. Cases $13,14,23$ and 24) at each of the four vertical locations followed by the simple average of those four stations. The final rows of the tables show the approximate computational cost relative to 
Case 13 which is the coarsest of the grid-converged simulations. Observations to be made from the error tables for this low Reynolds number flow are very similar to those previously discussed for the high Reynolds number flow. Overall the errors are quite small (for tubulence intensity even smaller than for the backward facing step cases) but the same trends exist; namely that errors decrease with a decrease in the filter width, and for a fixed computational cost (c.f. Cases 14 and 23) one is better off by choosing a smaller filter width while maintaining the FGR at the minimum value required to achieve grid convergence. More so than in the high Reynolds number simulations, there appears to be very little benefit in this cavity case to increase FGR beyond a value of 8 as seen by Case 24 which produces much the same overall error as Case 23 while costing 16 times as much.

Table 5.3. Relative mean error analysis of mean velocity and dimensionless temperature for

Case 13 and Case 14 for cavity flow

\begin{tabular}{|c|c|c|c|c|}
\hline \multirow{2}{*}{$\begin{array}{c}\text { Test locations } \\
(z / H)\end{array}$} & \multicolumn{4}{|c|}{ Mean velocity } \\
\cline { 2 - 5 } & $\begin{array}{c}\text { Case 13 }(\%) \\
\delta \mathrm{x}=0.125 \Delta_{1}, \mathrm{FGR} 8\end{array}$ & $\begin{array}{c}\text { Case 14 }(\%) \\
\delta \mathrm{x}=0.0625 \Delta_{1}, \mathrm{FGR} 16\end{array}$ & $\begin{array}{c}\text { Case 23 }(\%) \\
\delta \mathrm{x}=0.125 \Delta_{2}, \mathrm{FGR} 8\end{array}$ & $\begin{array}{c}\text { Case 24 }(\%) \\
\delta \mathrm{x}=0.0625 \Delta_{2}, \mathrm{FGR} 16\end{array}$ \\
\hline 0.765 & 2.88 & 3.02 & 3.15 & 3.11 \\
\hline 0.50 & 3.79 & 3.12 & 3.13 & 2.91 \\
\hline 0.229 & 4.25 & 3.91 & 2.46 & 2.38 \\
\hline 0.096 & 4.13 & 3.83 & 3.18 & 3.18 \\
\hline Average & 3.76 & 3.47 & 2.98 & 2.90 \\
\hline Relative cost & 1 & 16 & 16 & 256 \\
\hline
\end{tabular}

Table 5.4. Relative mean error analysis of mean velocity and dimensionless temperature for Case 23 and Case 24 for cavity flow

\begin{tabular}{|c|c|c|c|c|}
\hline \multirow{2}{*}{$\begin{array}{c}\text { Test locations } \\
(z / H)\end{array}$} & \multicolumn{4}{|c|}{ Non-dimensional temperature } \\
\cline { 2 - 5 } & $\begin{array}{c}\text { Case 13 }(\%) \\
\delta \mathrm{x}=0.125 \Delta_{1}, \mathrm{FGR} 8\end{array}$ & $\begin{array}{c}\text { Case 14 }(\%) \\
\delta \mathrm{x}=0.0625 \Delta_{1}, \mathrm{FGR} 16\end{array}$ & $\begin{array}{c}\text { Case 23 }(\%) \\
\delta \mathrm{x}=0.125 \Delta_{2}, \mathrm{FGR} 8\end{array}$ & $\begin{array}{c}\text { Case 24 }(\%) \\
\delta \mathrm{x}=0.0625 \Delta_{2}, \text { FGR 16 }\end{array}$ \\
\hline 0.765 & 2.87 & 2.35 & 2.41 & 2.93 \\
\hline 0.50 & 3.51 & 3.47 & 2.83 & 2.81 \\
\hline 0.229 & 3.39 & 2.76 & 2.65 & 2.12 \\
\hline 0.096 & 2.39 & 2.41 & 2.17 & 2.15 \\
\hline Average & 3.04 & 2.75 & 2.52 & 2.50 \\
\hline Relative cost & 1 & 16 & 16 & 256 \\
\hline
\end{tabular}




\subsubsection{Prominent flow features}

Figures 5.16 and 5.17 present snapshots of the velocity and temperature fields in the low Reynolds number flow as it develops in the differentially heated cavity. Results are for Case 23 which is the finer filter width case with FGR $=8$. Turbulent buoyancy driven flows in differentially heated cavities exhibit several regions in which the flow separates from the walls and is becomes recirculatory. This has a profound effect on the overall dynamics of the cavity, and it provides a somewhat demanding test of the effectiveness of the explicit approach to LES taken in this work. Lau et al. (2013) formulated dynamic sub-grid LES models to simulate Cheesewright et al. (1986) experiments on the flow described in this work. Their work indicated the presence of a small eddy in the upper corner of the enclosure, and located between the heated wall and the upper adiabatic surface. The average diameter of the eddy is about $0.02 \mathrm{~W}$. This small eddy is clearly captured in our work, as can be seen from the instantaneous flow field presented in figure 5.16(c), and it has dimensions that are of a similar magnitude to that described by Lau et al. (2013).

The reversal in the direction and the changes in magnitude of the velocity vectors also evident in figure 5.16(c) indicate that a second recirculation region exists in close proximity to the upper wall of the cavity. At the instant represented in the figure it is in the vicinity of about $0.3 \mathrm{~W}$ from the heated wall and $0.2 \mathrm{~W}$ from the upper adiabatic surface. This is very close agreement with the findings of Lau et al. (2013) who observed a similar phenomenon and this further vindicates the validity of the explicit filtering scheme presented in this paper. As the fluid flows in the upper region of the cavity towards the cooled vertical wall it relaminarises before it descends under the influence of gravity. This general behaviour is reflected in figure 5.16, and it is interpreted by Lau et al. (2013) as resulting from the upper adiabatic surface acting as a sink for turbulence, thus reducing the production of turbulent kinetic energy. 


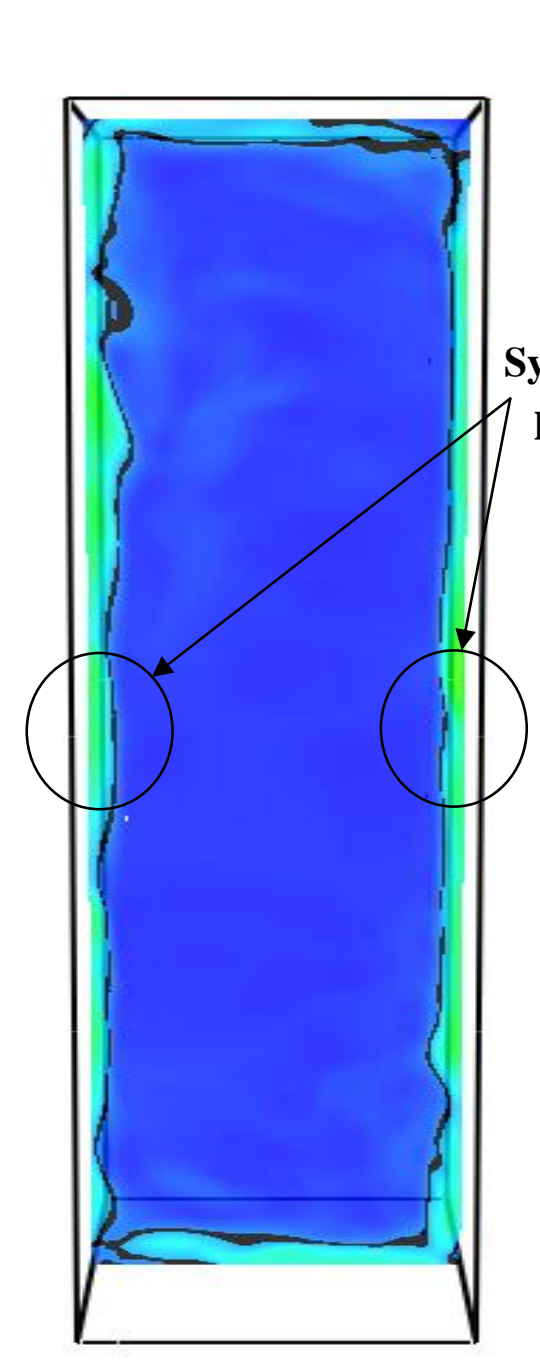

(a)

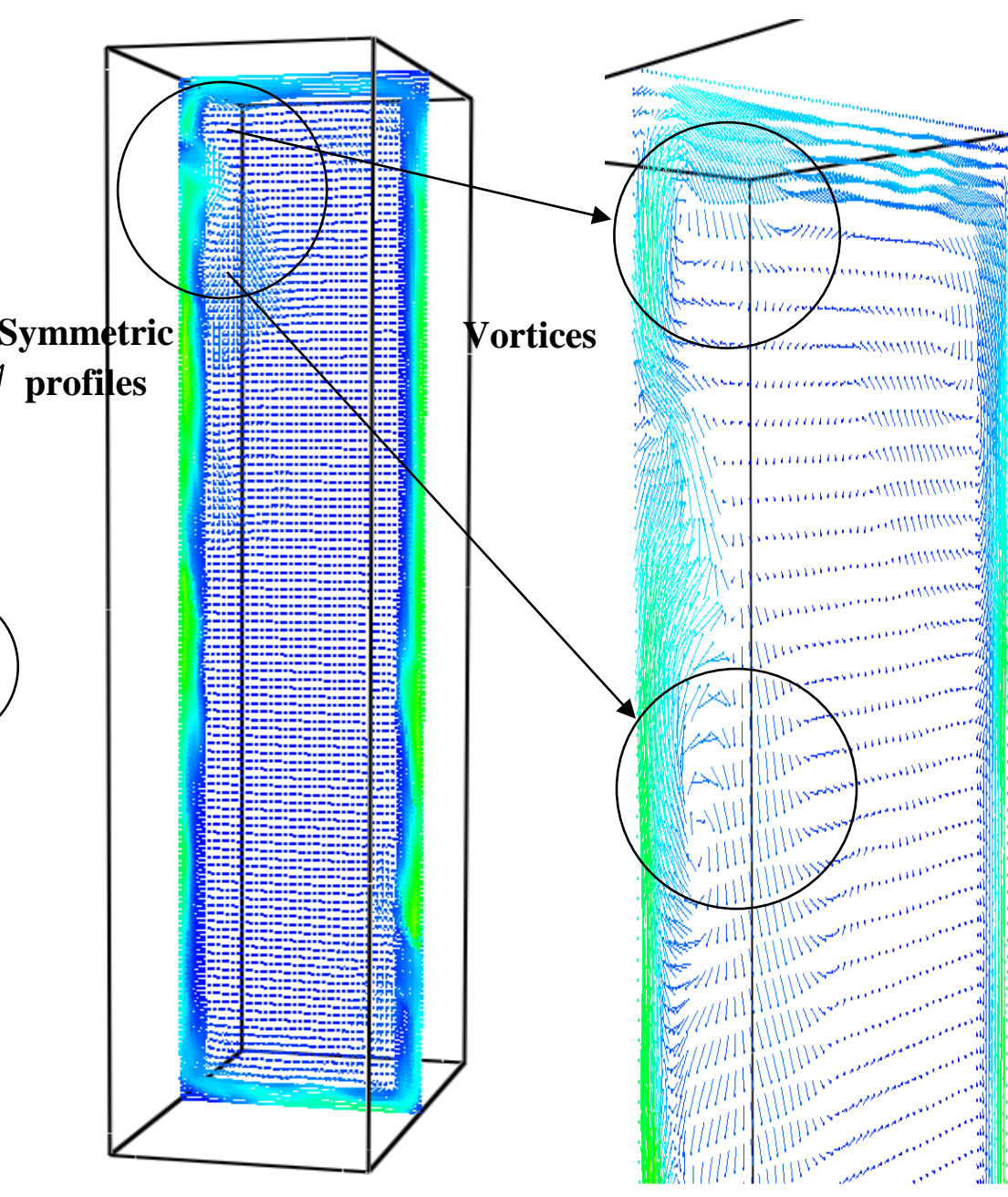

(b)
Slice

$\mathrm{m} / \mathrm{s}$

0.56

0.51

0.45

0.40

0.34

0.28

0.23

0.17

0.11

0.06

(c)

Figure 5.16. Snapshot of the shaded streamline plot (a) and vector plots (b) \& (c) of velocity inside the differentially heated cavity along the midsection at quasi-steady state after approximately $150 \mathrm{~s}$. Results are for Case 23 


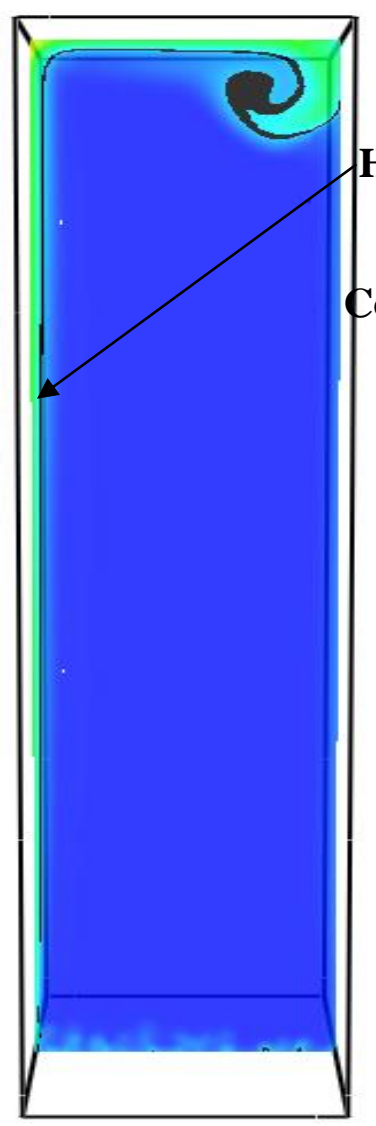

(a)

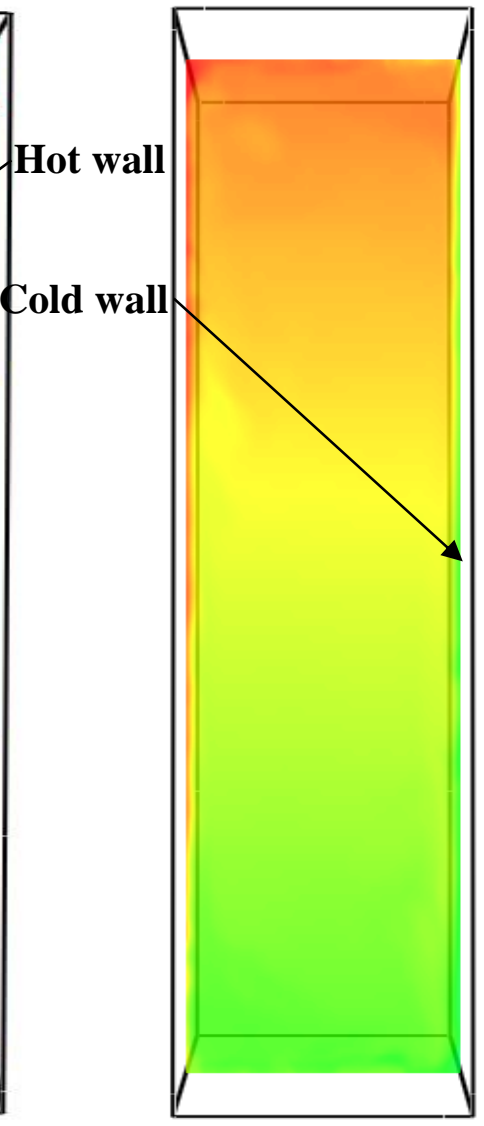

(b)

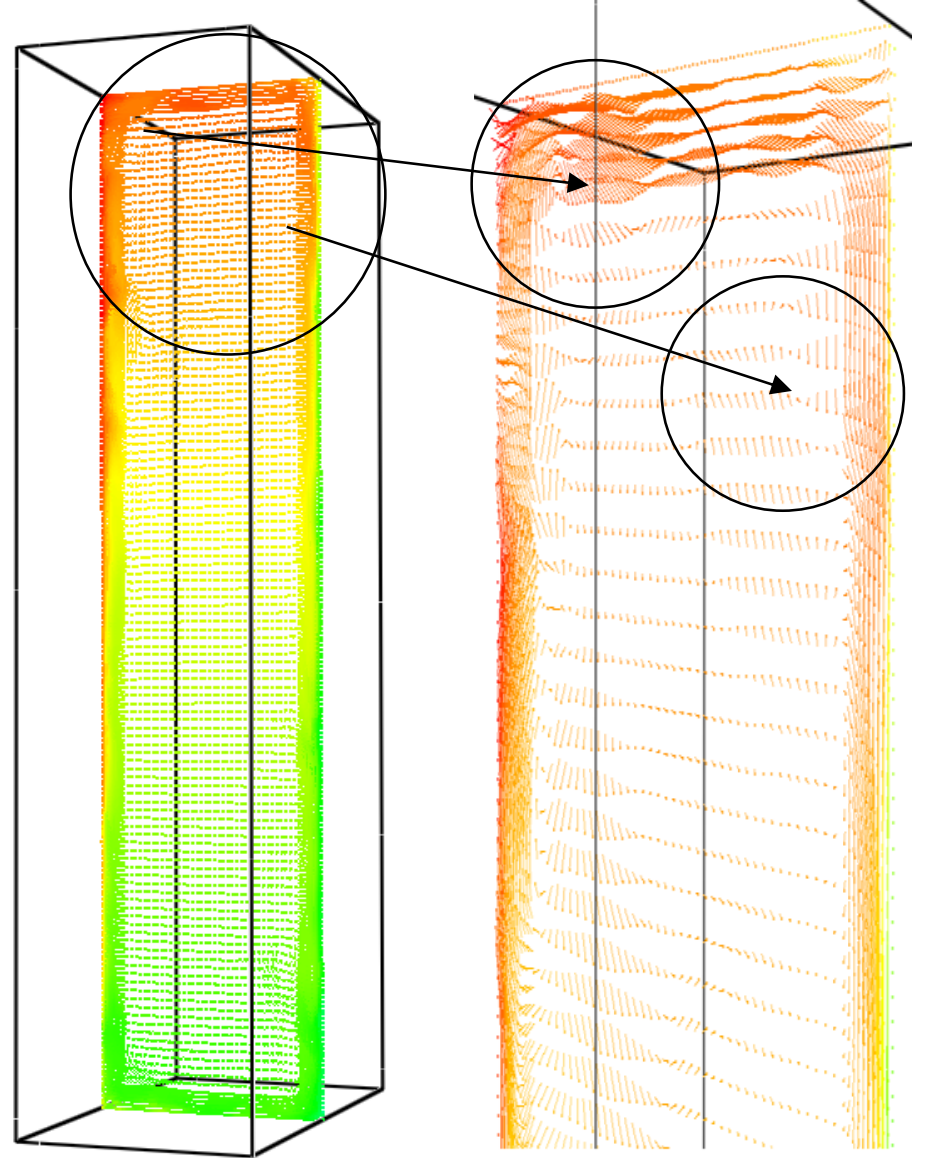

(c)

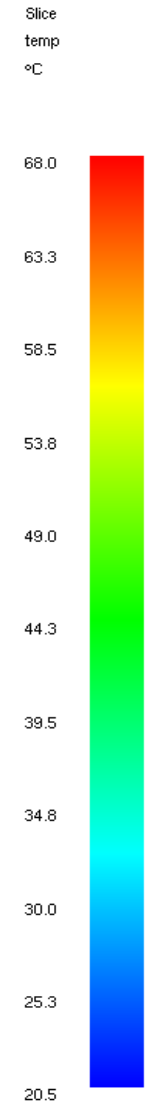

(d)

Figure 5.17. A snapshot of the isotherms plots obtained after $2 \mathrm{~s}$ of initiating the experiment, (b) depicts the temperature distribution at the quasi steady state. (c) \& (d) that also indicate the quasi-steady state temperature and flow field on the mid-section of a differentially heated cavity. Results are for Case 23 after approximately $150 \mathrm{~s}$ 
The experimental studies of Ozoe et al. (1985) demonstrate that eddies form close to the heated wall of differentially heated cavities (they did not present data for flow adjacent to the cooled wall). In their work they studied buoyancy driven flows of water at a Rayleigh number of $6.3 \times 10^{10}$ and they observed that perturbations in the mean flow, indicating the formation of eddies, occurring up to $20 \mathrm{~mm}$ from the heated wall. Figure 5.16(c) shows that in our work large eddies are predicted to be generated along both the heated and cooled walls, and it can be observed that the centres of the eddies are approximately $0.2 \mathrm{~W}$ from the walls. This is in close agreement with the observations made by Lau et al. (2013), which again by the principle of consilience provides support for the explicit filtering process presented in this work. The production of eddies, particularly in the upper region of the heated wall and lower region of the cooled wall as indicated in figure 5.17(c) promotes the thermal stratification that is also evident in figure 5.17. Overall, it appears that solution with the smaller filter width $10 \%$ of BLT and FGR 8 i.e. Case 23, captures the detailed physics of prominent flow features within the differentially heated cavity.

\subsection{Conclusions}

A systematic explicitly filtered large eddy simulation (LES) is used to simulate buoyancy driven turbulent flow due to natural convection inside a differentially heated rectangular cavity. This widely used benchmark is considered a low Reynolds number test case with $R e_{\lambda} 25$. While conducting energy spectra analysis, focus is given on the necessity to consider whether selected filter widths $\Delta_{1}$ and $\Delta_{2}$ fulfil the criteria for satisfying the principle of LES by capturing the energy containing length scales of eddies. In this test case, inertial subrange does not approach the theoretical slope like high $R e$ case which shows somewhat lack of LES principle. From the energy spectrum analysis and comparison with experimental results, it appears that solution with 
the smaller filter width $10 \%$ of BLT and FGR 8 i.e. Case 23 is more likely to provide an appropriate solution in an explicit scheme with optimal computational resources. By presenting energy spectra analysis it also justifies the important fact that low Reynolds number turbulent flow do not necessarily follow the $-5 / 3$ Kolmogorov universal scaling for inertial subrange and the range is very small that hardly exist in the spectra as the large and small scale lengthscales are difficult to separate. Overall error analysis and good agreement of simulation flow variables (i.e. mean velocity and temperature) results with experimental reference data also justifies that the smaller filter width with $10 \%$ of BLT and FGR 8 is sufficient for obtaining an appropriate solution for low Reynolds number turbulent flow inside the cavity. In addition to these, the prominent flows feature presentation of this flow shows that all essential physics of the flow can be captured properly. 


\section{Chapter 6}

\section{Conclusions and future work}

\subsection{Conclusions}

The inexorable progress of engineering science enables us to analyse systems in increasing spatial and temporal detail. For example large eddy simulation (LES) provides engineers and scientists with a powerful practical tool to evaluate the effects of eddies generated by turbulent flows. This is particularly important when we wish to study in detail how heat mass and momentum are transformed by eddies in the flow. The significant contribution of this study is the introduction of a new concept to select the filter in an explicit LES scheme based on physical parameter boundary layer thickness (BLT) to simulate wall-bounded turbulent flows. A systematic approach consistent with LES principles to obtain flow solutions using an explicit scheme integrating a damped standard Smagorinsky model is proposed and successfully applied to the benchmark case studies of high and low Reynolds number turbulent flows.

In this study, a second-order implicitly filtered LES code, Fire Dynamics Simulator (FDS), is taken as the baseline source code. A numerical study of a flow over a backward facing step has been simulated using four different SGS models in FDS to identify the most promising SGS model and to address the adequacy of a second-order numerical scheme. According to the results, the standard Smagorinsky eddy viscosity model is appeared to be the most accurate model out of four. It is shown that the Smagorinsky model has the highest degree of accuracy for a given mesh size. Analysis of the results of the further simulation with standard Smagorinsky model in terms of relative error and tendency of GCI justifies the adequacy of second-order numerical scheme. Having gained confidence in the second-order numerical scheme with the 
standard Smagorinsky model, this LES sub model with the addition of damped wall function along with the second-order scheme is modified to implement the explicitly filtered LES scheme discussed above.

Explicitly filtered LES offers the possibility of refining the numerical grid while maintaining the filter width constant, allowing for numerical errors to be minimised so that model performance may be assessed independently of the numerical error. While explicitly filtered LES schemes have been presented in the past they have often been for simple flows and the explicit filter width has been selected based on a priori DNS. In the present study explicitly filtered LES has been presented for two, relatively complex wall bounded flows. The first being a high Reynolds number flow over a backward facing step and the second a low Reynolds number flow in a differentially heated cavity. A systematic approach based on independent refinement of the explicit filter width and the grid resolution was suggested. In the absence of a priori DNS data, the filter for these wall bounded flows was initially set at $20 \%$ of a characteristic boundary layer thickness (BLT) and later refined to 10\%. This choice was made based on the boundary layer resolution suggestions found in the literature. As the BLT is a function of a streamwise coordinate, a particular reference point is chosen to determine BLT for each Re cases. For each of the two explicit filter settings the grid was progressively refined from a filter to grid size ratio (FGR) of two through to an FGR of 16.

Analysis of the energy spectra reveal, that for the two flow regimes, both explicit filter width settings can produce grid-converged results that are consistent with the principles of LES; namely that the filter lies within the inertial sub-range and the energy containing range is resolved. The former principle could be not be achieved for low Re flow due to the nature of the flow itself. From the energy spectrum analysis and comparison with experimental results, it 
appears that an appropriate solution can be obtained. For both filter widths this was achieved with an FGR of eight. This finding is at odds with some of the literature which suggests that for second-order numerical schemes an FGR of four is sufficient. The near-convergence of our simulations using an explicit filter of $10 \%$ of the BLT and FGR of four indicates that the required FGR is in fact filter width dependent with the possibility that smaller filters will permit lower values of the FGR.

Comparison against experimental data shows that the explicit LES scheme using a filter width of either $10 \%$ or $20 \%$ of BLT can capture the major features of the flow quite well with relative errors generally less than $5 \%$. The only shortcoming of note was the failure to accurately predict the near-wall velocity variance maxima downstream of the backward facing step. Comparison of the relative errors and computational load for the two different filter widths reveals that there is little benefit from increasing FGR above eight and that if resources permit greater grid-resolution then from the point of view of accuracy one is best off reducing the filter width while maintaining (or even slightly reducing) the FGR. Furthermore, this study shows the performance of explicit LES is much better than the implicit LES at coarser grid resolution which clearly demonstrate the advantage of using explicit LES over implicit LES to obtain accurate numerical solution.

\subsection{Future work and recommendations}

The two test cases numerically simulated in this study are important for their prominent flow features where fire is not involved. Hence, it is important to check the capability of the systematic explicit scheme that was developed for obtaining an appropriate and practical numerical solution for important flows where fire involved such as building-fires, grass fires and the lofting of firebrands by convection currents that arise from wildfires etc. Explicit filter width 
taken as 10\% of BLT and FGR of 8 is a good choice to obtain grid converged solution. However it is observed that one is best off reducing the filter width while maintaining (or even slightly reducing) the FGR, explicit filter width as 5\% of BLT and FGR of 4 can be explored. It is recommended that these suggested combinations of the filter with and FGR can be applied to small to medium scale scenarios involving compartment fires as well as vegetative fuel burning in landscapes. In landscape fire scenarios, the Australian grassfire experiment (Mell et al. 2007) can be considered as a relatively simple case to check the proposed scheme's ability to reproduce the experimental data. Furthermore, Simpson's rule with discrete and/ or Gaussian distribution to set the grid point weighting within filter width can be explored.

\subsection{Applications}

In the medium term, if the outcome of this study can lead to use the state-of-the art CFD-based fire model FDS for post fire analysis (both building and wildland fires) to obtain appropriate numerically grid converged solution without compromising accuracy, it will have a significant practical impact. Similarly, fire safety engineers may benefit by having a model capable of predicting the rate of spread and intensity of fires in buildings under a wide range of fire scenarios for designing fire protection systems. In the long term (the next generation), this study can lead to development of a real time bushfire modelling version to facilitate developing an early detection and warning system, as opposed to using simple empirical fire models. In future, this real time fire modelling tool can be used by first responders for tactical information on fire spread. 


\section{References}

Akselvoll, K, \& Moin, P 1993. Large eddy simulation of turbulent confined co-annular jets and turbulent flow over a backward facing step, RTF-63. Thermo-sciences Division, Dept of Mech. Engng, Stanford University.

Baldwin, BS, \& Lomax, H 1978, "Thin Layer Approximation and Algebraic Model for Separated Turbulent Flows", AIAA Paper 78, pp. 257.

Baldwin, BS, \& Barth, TJ 1990, A One-Equation Turbulence Transport Model for High Reynolds Number Wall-Bounded Flows, NASA TM 102847.

Balsara, DS, \& Shu, CW 2000, 'Monotonicity preserving weighted essentially non-oscillatory schemes with increasingly high order of accuracy', Journal of Computational Physics, vol. 160 , pp. 405-452.

Bardina J, Ferziger JH, \& Reynolds WC. 1980. Improved subgrid scale models for large eddy simulation. AIAA Journal, Paper- 80-1357

Bou-Zeid, E, Overney, J, Rogers, BD and Parlange MB, 2009, The Effects of Building Representation and Clustering in Large-Eddy Simulations of Flows in Urban Canopies, Boundary-Layer Meteorology, Vol 132, Issue 3, pp 415-436.

Brandt, T 2004, 'Studies on numerical errors in large eddy simulation', Licentiate's thesis, Helsinki University of Technology, Helsinki.

Carati, D., Winckelmans, G. \& Jeanmart, H. 2001. On the modelling of the subgrid-scale and filtered-scale stress tensors in large-eddy simulation. J. Fluid Mech. 441, 119-138.

Cebeci, T. and Smith, A.M.O. 1974. Analysis of turbulent boundary layers. Academic Press.

Cheesewright, R, King, KJ \& Ziai, S 1986, 'Experimental data for the validation of computer codes for the prediction of two-dimensional buoyant cavity flows', in ASME Winter Annual Meeting, HTD-60, Anaheim, pp. 75-81.

Choi, H \& Moin, P 2012, 'Grid-point requirements for large eddy simulation: Chapman's estimates revisited', Physics of Fluids, vol. 24, issue 1, pp. 011702 (1-5).

Chow, F.K \& Moin, P 2003, 'A further study of numerical errors in large eddy simulations', Journal of Computational Physics, vol. 184, pp. 366-380.

Clark, R, Ferziger, J \& Reynolds, W 1979. 'Evaluation of subgrid-scale models using an accurately simulated turbulent flow', Journal of Fluid Mechanics, vol. 91, pp. 1-16. 
Deardorff, J.W. 1970, A numerical study of three-dimensional turbulent channel flow at large Reynolds numbers, Journal of Fluid Mechanics, Vol. 41, pp. 453-480.

Deardorff, JW 1972, 'Numerical investigation of neutral and unstable planetary boundary layers, Journal of the Atmospheric Sciences, vol. 29, pp. 91-115.

Deardorff, JW 1973, 'The use of subgrid transport equations in a three-dimensional model of atmospheric turbulence', Journal of Fluids Engineering, vol. 95, pp. 429-438.

Djenidi, L, Antonia, RA, \& Tardu, S 2013, 'Breakdown of Kolmogorov's scaling in grid turbulence, in Proceedings of the 14th European Turbulence Conference, 1-4 September 2013, Lyon, France.

George, WK 2005, Lectures in Turbulence for the 21st Century, Department of Thermo and Fluid Engineering, Chalmers University of Technology, Gothenburg, Sweden, p. 64.

Germano M, Piomelli, U, Moin, P \& Cabot, WH 1991, 'A dynamic subgrid-scale eddy viscosity model', Physics of Fluids, A3, pp. 1760-1765.

Geurts, BJ \& Froehlich, J 2002, 'A framework for predicting accuracy limitations in large-eddy simulation', Physics of Fluids, vol. 14, no. 6, L41-L44.

Ghosal, S \& Moin, P 1995, 'The basic equations of the large eddy simulation of turbulent flows in complex geometry', Journal of Computational Physics, vol. 118, pp. 24-37.

Ghosal, S 1996, 'An analysis of numerical errors in large-eddy simulations of turbulence', Journal of Computational Physics, vol. 125, pp. 187-206.

Glendening JW, Haack, T 2001, 'Influence of advection differencing error upon large-eddy simulation accuracy', Boundary Layer Meteorology, vol. 98, no.1, pp. 127-153.

Gullbrand, J 2001, 'Explicit filtering and subgrid-scale models in turbulent channel flow', Annual Research Briefs, Center for Turbulence Research, Stanford University, pp. 31-42.

Gullbrand, J 2002, 'Grid-independent large-eddy simulation in turbulent channel flow using three-dimensional explicit filtering', Annual Research Briefs, Center for Turbulence Research, Stanford University, pp. 167-179.

Gullbrand, J 2004, 'Dynamic modeling in large-eddy simulation of turbulent channel flow, investigation of two-dimensional versus three-dimensional test filtering, International Journal of Numerical Methods for Heat \& Fluid Flow, vol. 14, no. 4, pp. 467-492.

Gullbrand, J \& Chow, FK 2003, 'The effect of numerical errors and turbulence models in largeeddy simulations of channel flow, with and without explicit filtering', Journal of Fluid Mechanics, vol. 495, pp. 323-341. 
Harlow, FH \& Welch, JE 1965. Numerical Calculation of Time-dependent Viscous Incompressible Flow of Fluid with Free Surface, Physics of Fluids vol. 8: pp 2182-89.

Holman, JP 2004, Heat Transfer, 9th edn, McGraw Hill Press.

Ierardi, JA, Barnett, JR 2003, 'A quantitative method for calibrating CFD model calculations'. Proceedings of the CIB-CTBUH international conference on tall buildings, International Council for Research and Innovation in Building and Construction (CIB), pp. 507-514.

Isomoto K, \& Honami S 1989. The effect of inlet turbulence intensity on the reattachment process over a backward-facing step, ASME Transactions Journal of Fluids Engineering, vol.111, pp 87-92.

Jones, WP, \& Launder, BE 1972, 'The prediction of laminarization with a two-equation model of turbulence', International Journal of Heat and Mass Transfer, vol. 15, pp. 301-314.

Jovic, S \& Driver, DM 1994, 'Backward-facing measurement at low Reynolds number $\mathrm{Re}_{\mathrm{h}}=$ 5000', NASA Technical Memorandum 108807, pp. 1-24.

Kempf, A, Geurts, B, \& J. Ofelein, 2011, Error analysis of large-eddy simulation of the turbulent non-premixed Sydney bluff-body flame, Combustion and Flame, vol. 158, pp. 2408-2419.

Keskinen JP, Vuorinen V, Kaario O, Larmi M, 2016. Influence of mesh deformation on the quality of large eddy simulations. International Journal for Numerical Methods in Fluids, Pape ID: 2712.

Kraichnan RH. 1976, Eddy viscosity in two and three dimensions. Journal of Atmospheric Science Vol 33:1521-36.

Kim, J, Moin, P \& Moser, R 1987, 'Turbulence statistics in fully developed channel flow at low Reynolds number', Journal of Fluid Mechanics, vol. 177, pp. 133-166.

Kolmogorov, AF 1941, 'On degeneration of isotropic turbulence in an incompressible viscous fluid', Comptes Rendus (Doklady) de l'Academie des Sciences del'URSS, vol. 31, no. 6, pp. $538-540$.

Kolmogorov, AF 1941, 'The local structure of turbulence in incompressible viscous fluid for very large Reynolds numbers', Comptes Rendus (Doklady) del'Academie des Sciences de l'U R-SS, vol. 30, no. 4, pp. 301-305.

Kravchenko, AG \& Moin, P 1997, 'On the effect of numerical errors in large eddy simulations of turbulent flows', Journal of Computational Physics, vol. 131, pp. 310-322. 
Kravchenko, A \& Moin, P 2000, 'Numerical studies of flow over a circular cylinder at $\mathrm{Re}_{\mathrm{d}}=$ 3900', Physics of Fluids, vol. 12, pp. 403-417.

Kuehn, DM 1980. Effects of Adverse Pressure Gradient on the Incompressible Reattaching Flow over a Rearward-Facing Step, AIAA Journal, vol.18, 343-344.

Laufer, J, 1954, The structure of turbulence in fully developed pipe flow, NACA, TRN-1174.

Lau, GE, \& Yeoh, GH, Timchenko V \& Reizes JA 2013, 'Large-eddy simulation of turbulent buoyancy-driven flow in a rectangular cavity', International Journal of Heat and Fluid Flow, vol. 39, pp. 28-41.

Launder, BE, \& Sharma, BI 1974, 'Application of the energy dissipation model of turbulence to the calculation of flow near a spinning disc', Letters in Heat and Mass Transfer, vol. 1, no. 2, pp. 131-138.

Launder, BE, Reece, GJ, \& Rodi, W 1975, 'Progress in the Development of a Reynolds-Stress Turbulent Closure.', Journal of Fluid Mechanics, vol. 68(3), pp. 537-566.

Le, H, \& Moin, P 1997, 'Direct numerical simulation of turbulent flow over a backward facing step', Journal of Fluid Mechanics, vol. 330, pp. 349-374.

Le, M \& Moser, RD 2015, Direct numerical simulation of turbulent channel flow up to $\mathrm{Re}_{\tau} \sim 5200$, Journal of Fluid Mechanics (accepted).

Leslie, D.C. \& Quarini, G.L. 1979, The application of turbulence theory to the formulation of subgrid modelling procedures, Journal of Fluid Mechanics, Vol. 91, pp. 65-91.

Lesieur M \& Metais O. 1996, New trends in large-eddy simulations of turbulence. Annual Review of Fluid Mechanics. Vol 28:45-82.

Leonard, A 1974. 'Energy cascade in large-eddy simulations of turbulent fluid flows', Advances in Geophysics A, vol. 18, pp. 237-248

Libby, PA 1996, Introduction to Turbulence, Taylor \& Francis, Washington, DC.

Lilly, D.K. 1966, The representation of small-scale turbulence in numerical simulation experiments, in Proc. IBM Scientific Computing Symposium on Environmental Sciences (NCAR), Paper No. 281.

Lilly, DK 1992, 'A proposed modification of the Germano subgrid-scale closure method', Physics of Fluids A, vol. 4, pp. 633-635. 
Lund, TS 1997, 'On the use of discrete filters for large eddy simulation', Annual Research Briefs, Center for Turbulence Research, NASA/Ames, Stanford University, pp. 83-95.

Lund, TS \& Kaltenbach, HJ 1995, 'Experiments with explicit filtering for LES using a finitedifference method', Annual Research Briefs, Center for Turbulence Research, Stanford University, pp. 91-105.

Majander, P \& Siikonen, T 2002, 'Evaluation of Smagorinsky-based sub grid scale models in a finite-volume computation', International Journal for Numerical Methods in Fluids, vol. 40, pp. 735-774.

Mansour, N \& Wray, A 1994, 'Decay of isotropic turbulence at low Reynolds number, Physics of Fluids, vol. 6, no. 2, pp. 808-814.

Mahesh, K, Constantinescu, G, Apte, S, Ham, F \& Moin, P 2006, 'Large-eddy simulation of reacting turbulent flows in complex geometries', Journal of Applied Mechanics, vol. 73, no. 3, pp. 374-382.

Mathew, J, Lechner, R, Foysi, H , Sesterhenn, J \& Friedrich R, 2003. An explicit filtering method for large eddy simulation of compressible flows, Physics of Fluids, vol. 15, pp 2279.

McGrattan, KB, Klien, B, Hostikka, S \& Floyd, J 2011, Fire Dynamics Simulator Version 6: Technical Reference Guide, National Institute of Standards and Technology, Special Publication, pp. 1018-6.

McDermott, RJ 2014. A velocity divergence constraint for large-eddy simulation of low-mach flows, Journal of Computational Physics, vol. 274, pp. 413-431.

Mell, W, Jenkins, MA, Gould, J 2007, 'A physics-based approach to modelling grassland fires', International Journal of Wildland Fire, vol. 16, pp. 1-22.

Mell, W, Manzello, SL, \& Maranghides, A 2010, 'The wildland-urban interface fire problem current approaches and research needs', International Journal of Wildland Fire, vol. 19, pp. 238-251.

Mell, W, Maranghides, A, McDermott, R \& Manzello, SL 2009, 'Numerical simulation and experiments of burning Douglas fir trees', Combustion and Flame, vol. 156, pp. 20232041.

Meneveau, C 1994, 'Statistics of turbulence subgrid-scale stresses: necessary conditions and experimental tests', Physics of Fluids, A, no. 6, p. 815. 
Meneveau C, Lund TS \& Cabot WH. 1996. A Lagrangian dynamic subgrid-scale model of turbulence. Journal of Fluid Mechanics, Vol 319:353-85.

Meyers, J, Geurts, BJ \& Baelmans, M 2003, 'Database analysis of errors in large-eddy simulation', Physics of Fluids, vol. 15, no. 9, pp. 2740-2755.

Meyers, J \& Sagaut, P 2007, 'Is plane-channel flow a friendly case for the testing of large-eddy simulation subgrid-scale models?', Physics of Fluids, vol. 19, pp. 048105 (1-4).

Moin, P, Squires, K, Cabot, W \& Lee, S 1991, 'A dynamic subgrid-scale model for compressible turbulence and scalar transport', Physics of Fluids, A3, pp. 2746-2757.

Moinuddin, KAM, Prasannan, K, Lalunio, R \& Thorpe GR 2010, 'Numerical modelling of fire spread in landscapes', 8th Asia-Oceania Symposium on Fire Science and Technology, Melbourne, Australia, pp. 1-11.

Morinishi, Y, Lund, TS, Vasilyev, OV, Moin, P 1998, 'Fully conservative higher order finite difference schemes for incompressible flow', Journal of Computational Physics, vol. 143, pp. 90-124.

Morinishi, Y, Vasilyev, O 1998, 'Subgrid scale modeling taking the numerical error into consideration', Annual Research Briefs, Center for Turbulence Research, NASA/Ames, Stanford University, pp. 237-253.

Najjar, FM \& Tafti, DK 1996, 'Study of discrete test filters and finite difference approximations for the dynamic subgrid-scale stress model', Physics of Fluids, vol. 8, pp. 1076-1088.

Neto, AS, Grand, D, M`etais O, \& Lesieur, M. 1993. A numerical investigation of the coherent vortices in turbulence behind a backward-facing step, Journal of Fluid Mechanics, vol.256, $1-25$.

Ozeo, H, Mouri, A.,Ohmuro, M., Churchill, S. W. and Lior, N. 1985. Numerical calculations of laminar and turbulent natural convection in water in rectangular channels heated and cooled isothermally on the opposing vertical walls, International Journal of Heat Mass Transfer, Vol. 28 pp 125-138

Panjwani, B, I. S. Ertesvag, A. Gruber, K. E. Rian 2010. Large eddy simulation of backward facing step flow, 5th National Conference on Computational Mechanics, Trondheim, Norway.

Paolucci, S, Chenoweth, DR 1989, 'Transition to chaos in a differentially heated vertical cavity', Journal of Fluid Mechanics, vol. 201, pp. 379-410. 
Piomelli, U, Moin, P \& Ferziger, JH 1988, 'Model consistency in large eddy simulation of turbulent channel flows', Physics of Fluids, vol. 31, pp. 1884-1891.

Pope, SB 2000, Turbulent flows, Cambridge University Press, Cambridge.

Pope, SB 2004. 'Ten questions concerning the large-eddy simulation', New Journal of Physics, pp. 6-35.

Prandtl, L 1925, 'Z. angew', Journal of Applied Mathematics and Mechanics, vol. 5, no. 1, pp. $136-139$.

Radhakrishnan, S, Bellan, J 2012, 'Explicit filtering to obtain grid-spacing-independent and discretization-order-independent large-eddy simulation of compressible single-phase flow, Journal of Fluid Mechanics, vol. 697, pp. 399-435.

Radhakrishnan, S, Bellan, J 2013, 'Explicit filtering to obtain grid-spacing-independent and discretization-order-independent large-eddy simulation of two-phase volumetrically dilute flow with evaporation', Journal of Fluid Mechanics, vol. 719, pp. 230-267.

Radhakrishnan, S, Bellan, J 2015, 'Explicitly-filtered LES for the grid-spacing-independent and discretization-order-independent prediction of a conserved scalar', Computers \& Fluids, vol. 111, pp. 137-149.

Reynolds, O 1895, 'On the dynamical theory of incompressible viscous fluids and the determination of the criterion', Proceedings of the Royal Society of London, Series A, vol. 186, pp. 123-164.

Richardson, LF 1922, 'The supply of energy from and to atmospheric eddies', Proceedings of the Royal Society of London, vol. 97, p. 686.

Richardson LF 1922, Weather prediction by numerical process, Cambridge University Press, Cambridge.

Roache PJ, 1992. Quantification of uncertainty in computational fluid dynamics, Annual Review Fluid Mechanics, vol. 29:123-60

Roache PJ, 1998. Verification and Validation in Computational Science and Engineering, Hermosa Publishers.

Rogallo RS \& Moin P. 1984, Numerical simulation of turbulent flows. Annual Review of Fluid Mechanics. 16:99-137.

Roy, CJ. 2003. Grid Convergence Error Analysis for Mixed-Order Numerical Schemes, AIAA Journal, vol. 41, pp no. 4. 
Sagaut, P 2001, Large eddy simulation for incompressible flows, Springer.

Schlichting, H 1979, Boundary-layer theory, McGraw Hill Press.

Schumann, U. 1975, Subgrid scale model for finite difference simulations of turbulent flows in plane channels and annuli, Journal of Computational Physics, Vol. 18, pp. 376-404.

Sharikin, A, 2015 Advancing Fire Simulation Through Development of a Fundamental Fire Property Database and its Optimised Use, Phd Thesis, Victoria University, Australia.

Smagorinsky, J 1963. 'General circulation experiments with the primitive equations', Monthly Weather Review, vol. 91, no. 3, pp. 99-164.

Spalart, PR, \& Allmaras, SR 1992, "A One-Equation Turbulence Model for Aerodynamic Flows", AIAA Paper 92-0439.

Sreenivasan, KR 1994, 'On the universality of the Kolmogorov constant', Physics of Fluids, vol. 7, no. 11 , pp. $2778-2784$.

Sullivan, AL 2009, 'Inland surface fire spread modelling, 1990-2007. 1: Physical and quasiphysical models', International Journal of Wildland Fire, vol. 18, pp. 349-368.

Taylor, GI 1935, 'Statistical theory of turbulence: Parts I-III', Proceedings of the Royal Society of London Series A, vol. 151, pp. 421-464.

Tennekes, H \& Lumley, JL 1972, A first course in turbulence, MIT Press.

Toms BA, 2015. Large-eddy Simulation of Flow over a Backward Facing Step: Assessment of Inflow Boundary Conditions, Eddy Viscosity Models, and Wall Functions, Journal of Applied Mechanical Engineering, vol. 4: pp(169).

Tsubokura, M 2001, 'Proper representation of the subgrid-scale eddy viscosity for the dynamic procedure in large eddy simulation using finite difference method', Physics of Fluids, vol. 13 , no. 2 , pp. 500-504.

Van Driest, ER 1956, 'On turbulent flow near a wall', Journal of Aeronautical Sciences, vol. 23, p. 1007.

Vasilyev, OV, Lund, TS \& Moin, P 1998, 'A general class of commutative filters for LES in complex geometries'. Journal of Computational Physics, vol. 146, pp. 82-104.

Vreman AW 2004, 'An eddy-viscosity subgrid-scale model for turbulent shear flow: algebraic theory and applications', Physics of Fluids, vol. 16, pp. 3670-3681.

Vreman, B, Geurts, B \& Kuerten, H 1994, 'Discretization error dominance over sub grid terms in large eddy simulation of compressible shear layers in 2D', Communications in Numerical Methods in Engineering, vol. 10, pp. 785-790. 
Vreman, B, Geurts, B \& Kuerten, H 1996, 'Comparison of numerical schemes in large-eddy simulation of the temporal mixing layer', International Journal for Numerical Methods in Fluids, vol. 22, p. 297.

H. Werner and H. Wengle. 1991 Large-eddy simulation of turbulent flow over and around a cube in a plate channel. In 8th Symposium on Turbulent Shear Flows, Munich, Germany, Technische University Munich, pages 155-168.

White. F. M. 2006. Fluid Mechanics. McGraw-Hill.

Wilcox, DC 1993, Turbulence modeling for CFD, DCW Industries, Inc., La Canada, CA.

Wilcox, DC 1997, Basic Fluid Mechanics, DCW Industries, Inc., La Canada, CA.

Winckelmans, G., Wray, A., Vasilyev, O. \& Jeanmart, H. 2001 Explicit-filtering large-eddy simulation using the tensor-diffusivity model supplemented by a dynamic smagorinsky term. Physics of Fluids, vol. 13, 1385-1403.

You, D, Wang, M, Moin, P \& Mittal, R 2007, 'Large eddy simulation analysis of mechanisms for viscous losses in turbo-machinery tip-clearance flow', Journal of Fluid Mechanics, vol. 586, pp. 177-204. 


\section{Appendix A}

\section{Details of Energy Spectra}

\section{A.1 Introduction}

The concept of energy spectra plays a crucial role in developing an LES scheme. Energy spectra provide detailed information on how the eddies evolve and transfer kinetic energies among themselves with different ranges of lengthscales in a system. Moreover, the effectiveness of LES schemes to obtain appropriate solutions are closely related to energy spectra analysis. In this appendix, the concepts of Reynolds number and energy spectra in turbulent flow are discussed in detail.

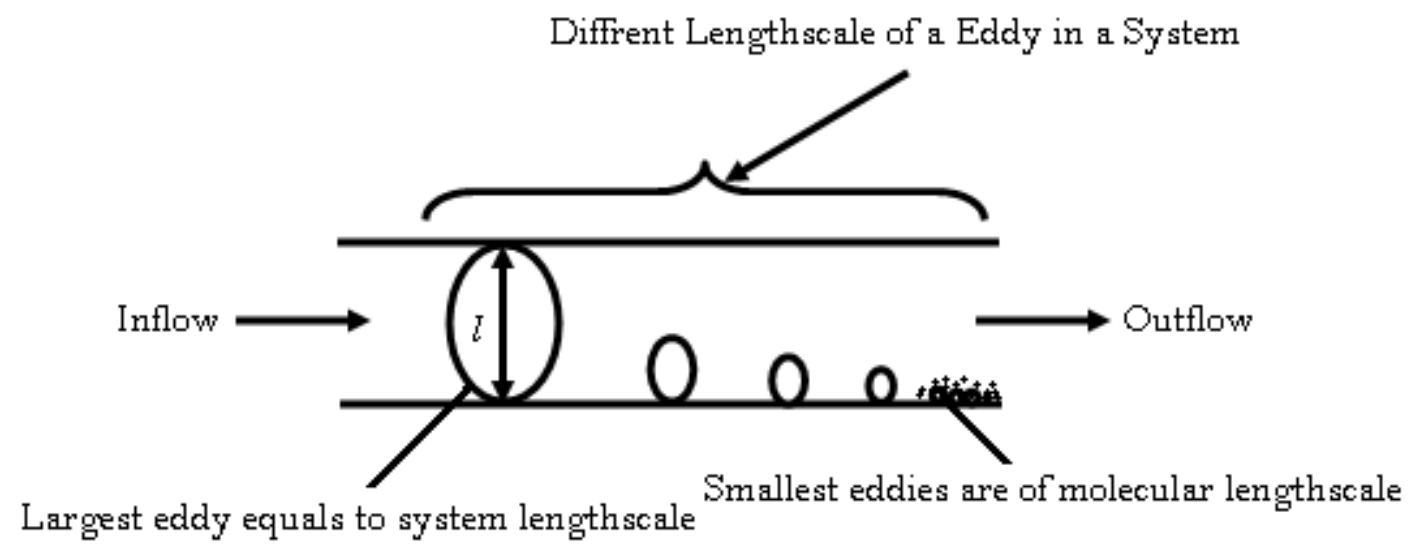

Figure A.1. Evolving lengthscales of an eddy in a system

\section{A.2 Concept of Reynolds number in turbulent flows}

Reynolds number is the most important parameter to define the nature of the flow and whether it is laminar or turbulent. In the case of laminar flow, the fluctuations of flow fields die out because of its steady and stable nature. On the other hand, in turbulent flow it originates because of its random nature. Turbulent flows are lumps of rotating fluid masses which are also known as 
eddies. There are eddies of different sizes in a turbulent flow. For example, if we consider a pipe flow like the one presented in Figure A.1, then we have a large eddy that will be of system lengthscale (here, it will be the equivalent of pipe diameter) and we can have small eddies of molecular lengthscale.

The large eddy extracts the energy from the mean flow because of instabilities in the mean flow. It can be said that mean flow has fluctuations with respect to time which actually triggers the turbulence. Because of these instabilities, the large eddy extracts energy from the mean flow. When the large eddy extracts energy from the mean flow, it will evolve into smaller and smaller eddies to which energy is subsequently passed. That means the energy will pass from the large eddy to the smaller eddy and the smaller eddy to smallest eddy of the molecular lengthscale. And, the entire energy cascade goes through smaller and smaller eddies till it is mopped up by the smallest eddies due to viscous dissipation. This energy cascading process is properly illustrated by the graphical representation among different eddy lengthscales (Figure 1.3) in section 1.3 of Chapter 1.

An important question to ask now is, why cannot large eddy energy be mopped up due to viscous dissipation and why do we have to go to the smallest eddy scale? Before we try to obtain an answer, we will first have to understand the differences in large scale and small scale eddies (presented in Table A.1).

Table A.1. Different parameters related to eddy scales

\begin{tabular}{|c|c|c|}
\hline Scales & Largest eddy & Smallest eddy \\
\hline Length scale & $l_{0}$ & $\eta$ \\
\hline Velocity scale & $u_{0}$ & $u_{\eta}$ \\
\hline Time scale & $t_{0}$ & $t_{\eta}$ \\
\hline
\end{tabular}

Here, if we know the lengthscale and velocity scale of different eddy scales we can easily determine the time scale. And, the lengthscale of the large eddy is the order of the system 
lengthscale. In addition to these, the transition behaviour from laminar to turbulent flow can be illustrated with the help of a Reynolds number which qualitatively represents the inertia to viscous forces. So, in this energy cascading process what is the significance of the Reynolds number? If the Reynolds number is either high or low, what does it actually mean?

What is meant is that with respect to the system lengthscale, the inertia force is dominant over the viscous force. Over the large eddy lengthscale the inertia force is much more dominant over the viscous force. That is, whatever the energy large eddy extracts from the mean flow that cannot be dissipated by the large eddy in the form of viscous dissipation. The viscous effects are negligible compared to the inertial effects for the large eddy. But when it comes to smaller and smaller eddies, the size or lengthscale of the eddy is small. That means the inertial force with the lengthscale will become smaller and smaller.

Compared to these, the Reynolds number is also becoming smaller with the reduced lengthscale. When we examine the smallest eddy, the Reynolds number will be just good enough to dissipate all the energy that has been cascaded from the larger eddy scale to the smaller eddy scale. So, it can be said that smallest eddies are good enough to dissipate all the energy through viscous dissipation. That means the smallest eddies will be characterized by a Reynolds number based on that lengthscale of the first order where the inertial force will be balance by the viscous force. So the Reynolds number will be to the order of 1 .

This can be by explained by the energy cascading process where the energy cascades down from the largest eddy to the smallest eddy. Let us first consider the rate of extraction of turbulent kinetic energy (TKE) from the mean flow,

$$
\pi \sim \frac{\text { Turbulentkineticenergy }}{\text { Timescale }}
$$


where, the largest eddies which will have energy of order $u_{0}^{2}$ and the turn-over time scale will be $t_{0}=l_{0} / u_{0}$, and it is a dynamically evolving phenomena. So,

$$
\pi \sim u_{0}^{3} / l_{0}
$$

And the rate of dissipation of turbulent kinetic energy at the smallest eddy scale,

$$
\varepsilon \sim \mathcal{v} e_{i j} e_{i j}
$$

where, $v$ is the kinematic viscosity and $e_{i j}$ is the rate of deformation tensor associated with the smallest eddy and the rate of deformation is given by velocity gradient at smallest eddy scale.

$$
\varepsilon \sim v\left(\frac{u_{\eta}}{\eta}\right)^{2}
$$

For having a dynamic balance, it must have the rate of extraction of turbulent kinetic energy (TKE) from the mean flow $(\pi)$ of the order of the rate of dissipation of turbulent kinetic energy $(\varepsilon)$ at the smallest eddy scale. So, whatever energy has been extracted from the large eddy scale the same energy will be dissipated and this process goes on in a repetitive way. So,

$$
\pi \sim \varepsilon
$$

another consideration is that the Reynolds number based on the smallest eddy lengthscale is the order of 1 . So, it can be written as,

$$
\begin{aligned}
& R e_{\eta} \sim 1 \\
& \frac{u_{\eta} \eta}{v} \sim 1
\end{aligned}
$$

By performing some algebraic manipulation in the above equations it is possible to determine the lengthscale of the largest and smallest scale eddy. The smallest scale of eddies can be seen as,

$$
\eta \sim\left(\frac{v^{3}}{\varepsilon}\right)^{1 / 4}
$$

So it can be said that the smallest eddy lengthscale does not depend on the characteristics of the largest eddy lengthscale; it only depends on the rate of dissipation of turbulent kinetic energy and the kinematic viscosity. This lengthscale is known as the Kolmogorov lengthscale. 
This Kolmogorov lengthscale can be related to the system lengthscale in the following way, from equation (A.4)

$$
\frac{u_{0}^{3}}{l_{0}} \sim v\left(\frac{u_{\eta}}{\eta}\right)^{2}
$$

After performing some algebraic manipulation the above equation can be presented as,

$$
\frac{\eta}{l_{0}} \sim R e_{l}^{-3 / 4}
$$

where, $R e_{l}$ is based on the system or largest eddy length scale. Now, from the above equation, in a similar way, for the velocity scales and the time scales of the smallest eddies compared to largest eddies it can be found that

$$
\begin{aligned}
& \frac{u_{\eta}}{u_{0}} \sim R e_{l}^{-1 / 4} \\
& \frac{t_{\eta}}{t_{0}} \sim R e_{l}^{-1 / 2}
\end{aligned}
$$

So, from the above correlations between the length, velocity and time scales compared to Reynolds number based on largest eddy, it appears that the higher the Reynolds number, the length, velocity and time scales will be varied with respect to the largest and smallest scales (Kolmogorov) of eddy. It also appears that with an increasing Reynolds number, fluid flows become more turbulent and lengthscales of eddies quickly evolve with respect to time. Therefore, it is important to capture all the length- and time scales to obtain the appropriate solutions during simulation of turbulent flows.

\section{A.3 The energy cascades}

In fluid flows, turbulence can take place in different scales ranging from large to smaller scales. And, these eddy scales decrease with the increase of the Reynolds number. Turbulent flow contains energy and distributes it in various scale levels of fluid flow motions. This is also known as the energy cascade system of turbulence. 
The idea of an energy cascade system was first introduced by Richardson (1922). Based on energy cascade theory, large eddies contain kinetic energy and they transfer this kinetic energy to the smallest scales. This process continues right up to the smallest scales of turbulence and at this stage energy is dissipated due to viscous action. According to Richardson, large eddies are unstable in nature and these eddies break up and form small eddies. During this time large eddies transfer energy into the small eddies. Similarly, these small eddies again break up and transfer their energy to comparatively smaller eddies. This process continues up to the smallest scale when the eddy motion becomes stable and the viscous effect is dominant. For instance, comparatively smaller eddies continue to transfer the energy until the Reynolds number $\operatorname{Re}(l) \equiv$ $u(l) * l / v$ is small enough that eddies are stable in nature.

The energy from the large eddies is transferred between the resolved and unresolved scales of motion, that means the net transfer of energy is from large to small scales. In addition, the smallest scale eddies of fluid motions are transferring energy by dissipation in the form of heat due to viscous effect. A question to raise here: where is the heat is going? The heat which is produced, which is hardly noticeable, is going into raising the temperature of the system.

In LES, dissipation of energy from the small scale eddies due to viscous effect can be considered as a source of energy and this phenomena is known as backscatter (Pope 2000). It is a challenging issue while designing the sub-grid closures in LES and it is often critical to account for the effect of these small scales in the large scale motions. In most simple LES cases, the subgrid closures consider that the production of sub-grid kinetic energy is equal to the total kinetic energy from dissipation.

The waves (in Fourier space) or eddies (in physical space) of different size, exchange energy with each other and they vary along three directions in space and time (Tennekes and Lumley 
1972). For example, eddies of size $l$, will have characteristic velocity $u(l)$ and the time scale is $\tau(l)=l / u(l)$. The rate of dissipation $(\varepsilon)$ can be obtained by counting the total amount of energy that is transferred by the largest eddies which will have energy of order $u_{0}^{2}$ and the time scale will be $\tau_{0}=l_{0} / u_{0}$ and the rate of energy transfer is equivalent to $u_{0}^{2} / \tau_{0}$. So, the rate of dissipation can be defined as $u_{0}^{3} / l_{0}$. At a high Reynolds number the effect of turbulent viscosity is ignored to predict the dissipation.

The energy cascade theory demonstrates how energy is transferred from large scale to small scale eddies though many questions about the size of eddies are unanswered. Moreover, the energy cascade theory is unable to answer which lengthscale of the smallest eddies is responsible for dissipating energy and cannot provide an answer for the relationship of velocity and timescale with decreased eddy length $l$. These questions are answered by Kolmogorov (1941) in stating three hypotheses. These three hypotheses are known as: the Kolmogorov's hypothesis of local isotropy, the first similarity hypothesis and the second similarity hypothesis.

\section{A.3.1 Hypothesis of local isotropy}

According to this hypothesis, at high Reynolds numbers, the small-scale eddies are isotropic in nature. It assumes that large scale eddies are anisotropic in nature and lie in the energy containing range: and the lengthscale of eddies is known as the integral lengthscale. If the large scale eddies length is considered as $l$, then it should be $l>l_{E I}$, where the demarcation of $l_{E I}$ separates the energy containing and inertial sub-range region. It is believed that, in the universal equilibrium range $\left(l<l_{E I}\right)$ large eddies break down and transfer the energy to the small eddies. In effect, in that range, all small eddies show a similar nature.

Moreover, they lose all directional information like turbulent flow at a high Reynolds number. In addition, this universal state can be explained by the two dominant processes of energy cascade 
theory. That means, large scale eddies break down successively transferring energy to the small scale and it takes place in the inertial sub-range. Whereas comparatively small scale eddies dissipate energy due to viscous $(v)$ effect which is more clearly demonstrated by his second hypothesis.

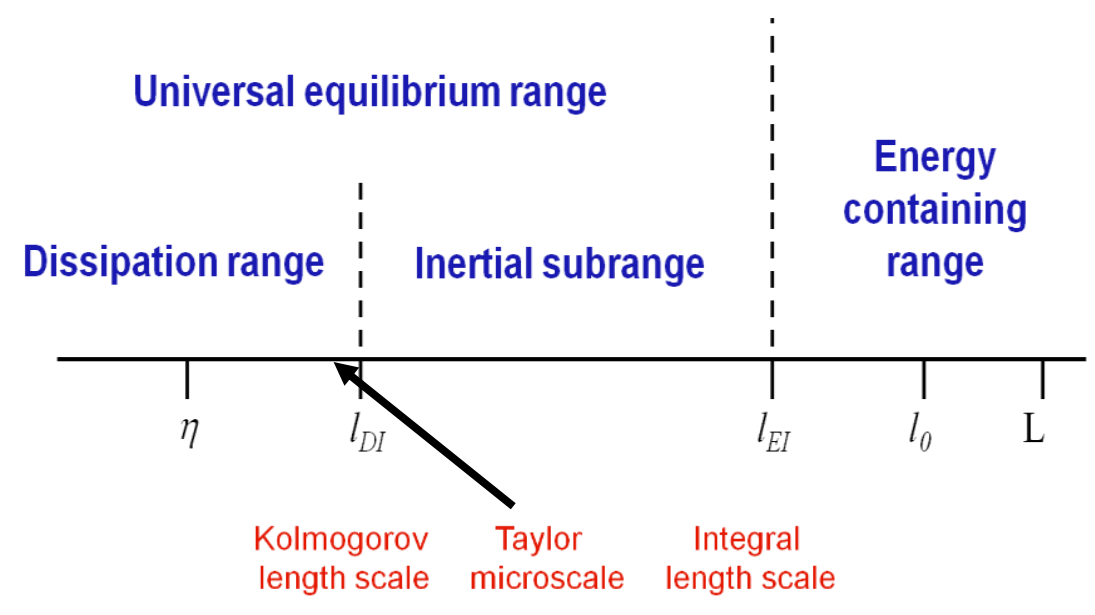

Figure A.2.Various ranges of turbulence at different lengthscales.

\section{A.3.2 First similarity hypothesis}

First similarity hypothesis presents the importance of small scale eddies in turbulent flow that can be uniquely defined by the viscosity of fluids and energy dissipated by the small eddies in the dissipation range. At a relatively high Reynolds number, eddies are more isotropic nature in the dissipation range.

'In every turbulent flow at sufficiently high Reynolds number, the statistics of the small scale motion $\left(l<l_{E I}\right)$ have a universal form that is uniquely determined by the viscosity $v$ and rate of energy dissipation $\varepsilon^{\prime}$ (Pope 2000).

From Figure A.2, the demarcation line $l_{E I}$ separates the energy containing range and the universal range. The large scale waves or eddies are consistent in the energy containing range and are anisotropic in nature along different directions with time. On the other hand, the energy 
cascading takes place in the universal range. Here, the large scale eddies break down and become smaller and smaller. These small eddies are isotropic in nature and exchange energy between each other. This eddy lengthscale is known as the Taylor's microscale. The Taylor microscale is an intermediate scale that is larger than the Kolmogorov scale but is small enough to be influenced by the viscous effect. DNS resolves up to the Kolmogorov scale but in practice that may not be possible. So, the DNS resolves down to the Taylor microscale. On the other hand, the LES must resolve down to the inertial subrange which is larger than the Taylor microscale. It assumes that at inertial sub-range viscous effect is negligible for dissipating kinetic energy.

In this range $\left(l<l_{E I}\right)$, the Kolmogorov length, velocity and time-scale can be determined by using viscosity and rate of dissipation. These parameters are also known as Kolmogorov's scales.

$$
\begin{gathered}
\eta \equiv\left(v^{3} / \varepsilon\right)^{1 / 4} \\
u_{\eta} \equiv(\varepsilon v)^{1 / 4} \\
\tau_{\eta} \equiv(v / \varepsilon)^{1 / 2}
\end{gathered}
$$

Moreover, these scales also provide the correlation between the dissipation and viscosity and characterize the velocity of the dissipative eddies (Pope 2000).

$$
\begin{gathered}
\varepsilon=v\left(u_{\eta} / \eta\right)^{2}=v / \tau_{\eta}{ }^{2} \\
u_{\eta}=\eta / \tau_{\eta}
\end{gathered}
$$

From Kolmogorov's scale, the ratios between the small scale and large scale eddies can be estimated in terms of a Reynolds number. Therefore, the following equations are again recalled from the previous section,

$$
\begin{gathered}
\eta / l_{0} \sim R e^{-3 / 4} \\
u_{\eta} / u_{0} \sim R e^{-1 / 4} \\
\tau_{\eta} / \tau_{0} \sim R e^{-1 / 2}
\end{gathered}
$$


It is clear from the above correlations that, at a high Reynolds number, velocity and time scales of the small eddies will be too small compared to large eddies. Moreover, the lengthscale $l$ in this range is small compared to $l_{0}$ (equivalent to system characteristic length) but relatively too large compared to the Kolmogorov's lengthscale. This hypothesis demonstrates that with increasing system lengthscale, fluid flows inherently become more turbulent in nature.

\section{A.3.3 Second similarity hypothesis}

This hypothesis provides information on the intermittent lengthscale of eddies in the universal equilibrium range which also separates the energy containing and dissipation range in the energy cascading process.

'In every turbulent flow at sufficiently high Reynolds number, the statistics of the motions of scale $l$ in the range $l_{0} \gg l \gg \eta$ have a universal form that is uniquely determined by the viscosity $v$ and rate of energy dissipation $\varepsilon^{\prime}$ (Pope 2000).

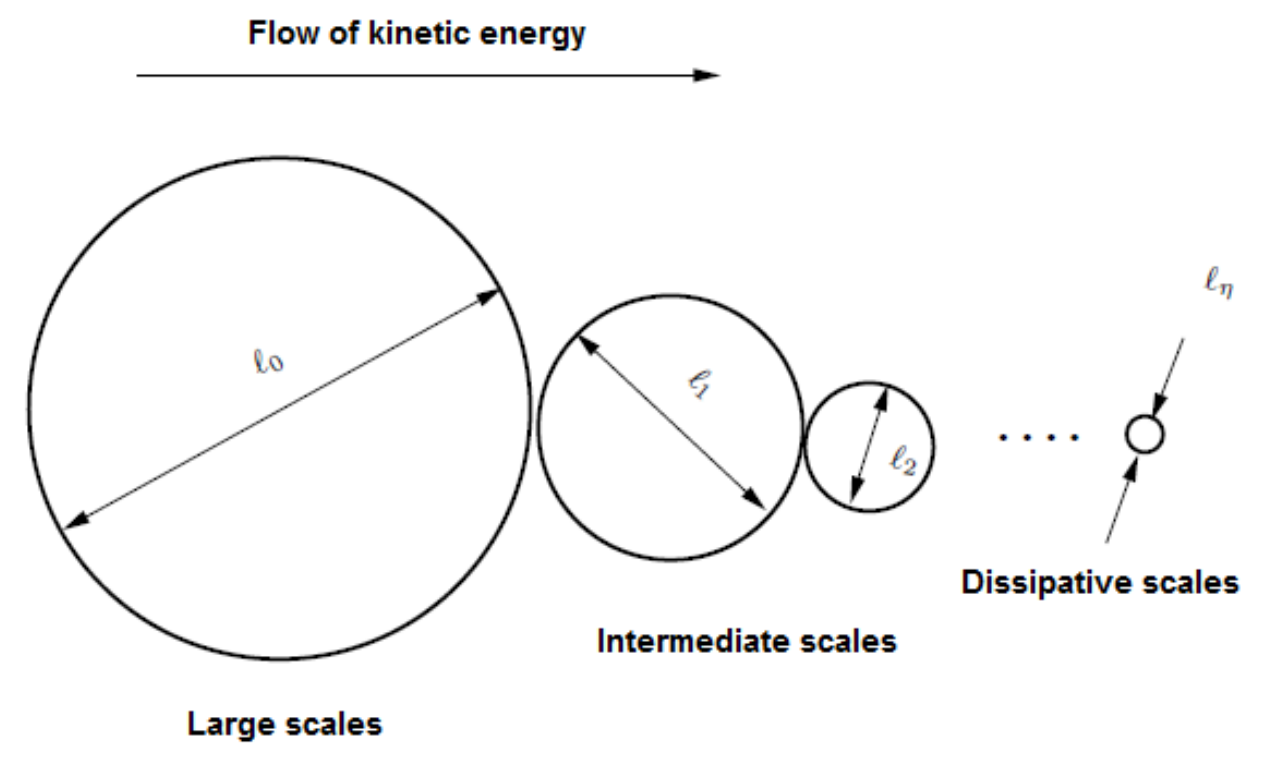

Figure A.3. Energy cascading with spectrum of eddies with different scales. 
From Figure A.2, it is found that in the universal equilibrium range, the length scale $l_{D I}$ separates the inertial and the dissipation range assumed $l_{D I}=60 \eta$ at high Reynolds number. So, the eddy with a lengthscale of $l$ in the inertial sub-range can be written as $l_{E I}>l>l_{D I}$ and the dissipation range as $l<l_{D I}$. The inertial sub-range considers the inertial effects of the flow which is independent of viscous effect. On the other hand, in the dissipation range, the viscous effects are dominant for all of the dissipation. Moreover, it assumes that the eddy size $l$ in the energy containing range would be $l_{E I}=\frac{1}{6} l_{0}<l<6 l_{0}$. This region consists of large eddies which contain bulk energy.

$$
l_{E I} \equiv T\left(l_{E I}\right)=T(l)=T_{D I} \equiv T\left(l_{D I}\right)=\varepsilon
$$

The different range of eddy scales shown in Figure A.3, shows how the energy is produced in the energy containing range and is distributed through the viscous effect in the dissipation range. It assumes that the rate of energy transfer from the large scale $T_{E I}$ to the small scale eddies is equivalent to the energy transfer to the inertial range $T(l)$. Furthermore, the energy leaving from the inertial range is equivalent to the energy entered in the dissipation range $T_{D I}$ and dissipated with rate $\varepsilon$ (Pope 2004). This hypothesis demonstrates an important fact: that production of energy in the largest scales in the energy containing range must be dissipated by the smallest scale eddies in the dissipation range by the viscous effect. The amount of energy production in the energy containing range should be equal to the amount of energy dissipation in the dissipation range. The inertial range is the intermittent range or universal range which helps to transfer the energy from large scale eddies to small scale eddies. Moreover, this hypothesis helps to simulate the turbulent flows with sufficient accuracy. It is believed that almost $80 \%$ energy is resolved if large scale eddies or the energy containing range is captured properly of any system 
(Pope 2000). Therefore, the LES principle encompasses requirements for setting its filter width in the inertial subrange and capturing all energy containing eddies properly.

\section{A.4 Kolmogorov's -5/3 law (theoretical slope)}

In turbulent flows, it is important to estimate how the turbulent kinetic energy due to velocity fluctuations is distributed among eddies with different lengthscales. An eddy with lengthscale $l$ and the corresponding wavenumber to this lengthscale of $\kappa=2 \pi / l$, and the energy contained among the wavenumbers that range from $\kappa_{a}$ to $\kappa_{b}$ can be expressed as,

$$
k_{\left(\kappa_{a}, \kappa_{b}\right)}=\int_{\kappa_{a}}^{\kappa_{b}} E(\kappa) d \kappa
$$

The contribution of the total dissipation rate $\varepsilon$ from the fluctuating part can be expressed as

$$
\varepsilon_{\left(\kappa_{a}, \kappa_{b}\right)}=\int_{\kappa_{a}}^{\kappa_{b}} 2 \nu \kappa^{2} E(\kappa) d \kappa
$$

The first similarity of Kolmogorov's hypothesis (Kolmogorov 1941) for the condition $\kappa>\kappa_{E I} \equiv$ $2 \pi / l_{E I}$ which falls in the universal equilibrium range and the energy spectrum function depends on the $v$ and $\varepsilon$ in this range. And, according to the second hypothesis the wavenumbers that fall in the inertial subrange $\kappa_{E I}<\kappa<\kappa_{D I} \equiv 2 \pi / l_{D I}$, the energy spectrum function is

$$
\begin{aligned}
& E(\kappa) \propto \varepsilon^{2 / 3} \kappa^{-5 / 3} \\
& E(\kappa)=C \varepsilon^{2 / 3} \kappa^{-5 / 3}
\end{aligned}
$$

The above equation describes the famous Kolmogorov $-5 / 3$ spectrum. Where, $C$ is the Kolmogorov constant which was experimentally determined as 1.5. For a deeper understanding of Kolmogorov's $-5 / 3$ law, let us consider the power law of a spectrum that can be written as

$$
E(\kappa)=A \kappa^{-p}
$$

where, $A$ and $p$ are constant. Now, the total energy contained by the wavenumbers that is greater than $\kappa$ is 


$$
k_{(\kappa, \infty)}=\int_{\kappa}^{\infty} E\left(\kappa^{\prime}\right) d \kappa^{\prime}=\frac{A}{p-1} \kappa^{-(p-1)}
$$

The equation is for $p>1$, but the integration diverges if $p \leq 1$.

On the other hand, the total dissipation for the wavenumbers less than $\kappa$ is

$$
\varepsilon_{(0, \kappa)}=\int_{0}^{\kappa} 2 v \kappa^{\prime 2} E\left(\kappa^{\prime}\right) d \kappa^{\prime}=\frac{2 v A}{3-p} \kappa^{3-p}
$$

The above equation converges for the condition $p<3$, while the integration diverges for $p \geq 3$.

In that case $p=\frac{5}{3}$ which lies in the middle of the range $(1,3)$ of the Kolmogorov spectrum will converge the integrals for both turbulent kinetic energy, $\kappa_{(\kappa, \infty)}$ and the amount of dissipation, $\varepsilon_{(0, \kappa)}$ will converge. 


\section{Appendix B}

\section{Simulation of Turbulent Pipe Flow}

\section{B.1 Introduction}

To solve the fluid flow problems numerically, three key elements are involved in computational fluid dynamics (CFD): numerical schemes and grid generation; algorithm development; and turbulence modelling. While for the first two elements rigorous mathematical formulations exist, turbulence modelling is less precise due to the complex nature of turbulent flows. Simulating turbulence is a fundamental problem of fluid dynamics. Therefore, before implementing an explicit scheme in a default version of FDS to simulate turbulent flows for preliminary simulations, two numerical algorithms (RANS codes) have been developed to solve a simple one-dimensional turbulent pipe flow. It is believed that the quality of turbulent flow simulations depend on the accuracy of eddy models to obtain appropriate numerical solutions. Two eddy models namely the Cebeci-Smith (1974) and $k-\varepsilon$ models are used in this study to account for the turbulence in the pipe flow. Furthermore, this study demonstrates the importance of eddy models along with numerical schemes for obtaining appropriate solutions of turbulent flows.

\section{B.2 Governing equations and boundary conditions}

Figure B.1 presents a fully developed flow inside a circular pipe. In the two-dimensional schematic diagram, $x$ and $y$ represents the streamwise and radial direction of the pipe. Flow inside the pipe can be laminar or turbulent depending upon the Reynolds number. For the preliminary simulations of one-dimensional turbulent flow inside the pipe, two Reynolds numbers $\left(R e_{\tau}\right)$ based upon friction velocity $\left(u_{\tau}\right)$ are considered: 180 and 395 respectively. 


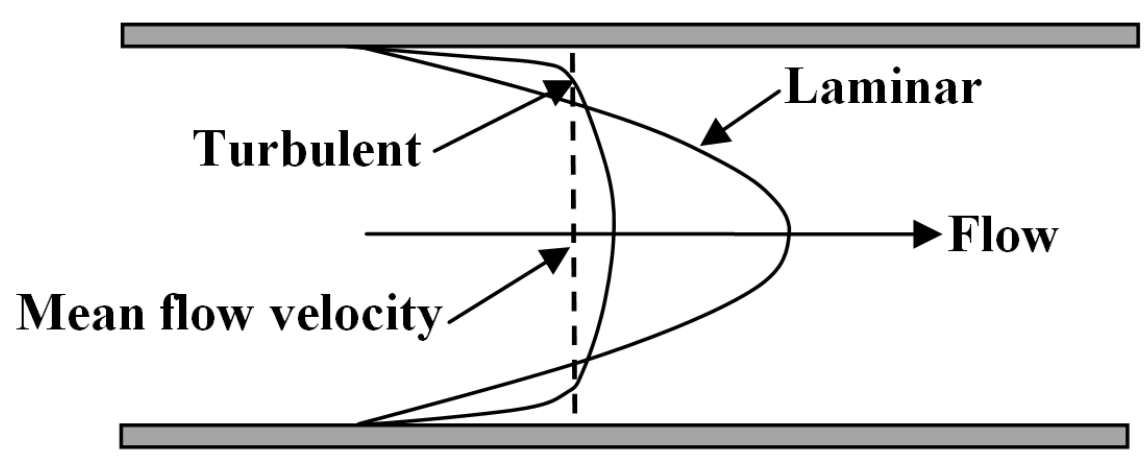

Figure B.1. Fully developed turbulent flow inside a circular pipe

The governing equation and the boundary conditions for the one-dimensional turbulent pipe flow are as follows,

$$
\left(v+v_{t}\right) \frac{\partial u}{\partial y}=1-y, \quad 0 \leq y \leq \alpha
$$

Boundary conditions are,

$$
\text { at } y=0, \quad u(0)=0, \quad \frac{\partial u}{\partial y}=0
$$

And,

$$
\text { at } y=\alpha, \quad u(\alpha)=\beta \text {; }
$$

where, $v$ is the kinematic viscosity, $v_{t}$ is the turbulent kinematic viscosity, $u$ is the velocity along the flow direction, $\alpha$ and $\beta$ are initial values that can be taken arbitrarily. Static kinematic viscosities are considered $0.00556 \mathrm{~m}^{2} / \mathrm{s}$ and $0.002532 \mathrm{~m}^{2} / \mathrm{s}$ for Reynolds numbers taken $\left(R e_{\tau}\right) 180$ and 395 respectively. The Reynolds number based on the frictional velocity is computed as $R e_{\tau}=u_{\tau} R / v ; R$ is the radius or centre line of the pipe where streamwise velocity is maximum $\left(u_{\max }\right)$. In these preliminary simulations, for the given Reynolds number, nondimensional velocity $u^{+}$has been plotted as a function of dimensionless distance $\left(y^{+}\right)$to the wall as, 


$$
u^{+}=f\left(y^{+}\right)
$$

where, $u^{+}=u / u_{\tau}$; mean velocity $(u)$ scaled to the wall friction velocity $\left(u_{\tau}\right)$ and dimensionless wall unit $y^{+}$is computed as $y u_{\tau} / \nu$. Here, the wall friction velocity is defined as $u_{\tau}=\sqrt{\tau_{\text {wall }} / \rho}$; where $\tau_{\text {wall }}$ is wall shear stress and $\rho$ is the density of the fluid which is considered unity for all simulations. Surface roughness is ignored and for all simulation inside the pipe surface is assumed smooth. The turbulent pipe flow is driven by the constant streamwise pressure gradient $(-\partial p / \partial x)$ which is considered 1 for all simulations.

\section{B.3 Numerical method}

For the simulations of turbulent pipe flow at various Reynolds numbers, non-uniform grid is used. To capture the detailed physics of fluid near the wall relatively fine meshes are used near the pipe wall and coarsen towards the radial direction of the pipe. The governing equation second-order finite difference scheme for one-dimensional grid points (presented in Figure B.2) is solved as follows,

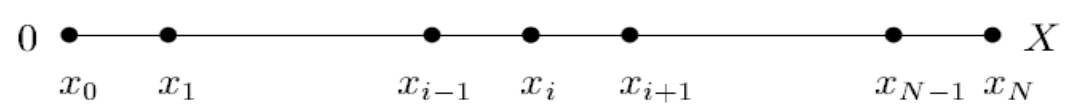

Figure B.2. Geometric arrangement of one-dimensional grid points

Derivatives in the partial differential equation are commonly computed by the linear combination of the function values at each of the assigned grid points and that can be written as,

$$
u_{i}=u\left(x_{i}\right), \quad i=0,1 \ldots \ldots . N
$$

For non-uniform grids, the following approximation of second-order derivatives of finite difference scheme has been used,

$$
\left(\frac{\partial^{2} u}{\partial x^{2}}\right)_{i}=\left[\frac{\partial}{\partial x}\left(\frac{\partial u}{\partial x}\right)\right]_{i}=\frac{\left(\frac{\partial u}{\partial x}\right)_{i+1 / 2}-\left(\frac{\partial u}{\partial x}\right)_{i-1 / 2}}{\partial x}
$$

After mathematical manipulation the above equation can be expressed as, 


$$
\left(\frac{\partial^{2} u}{\partial x^{2}}\right)_{i}=\frac{1}{\left(\frac{h_{1}}{2}+\frac{h_{2}}{2}\right)}\left(\frac{u_{i+1}-u_{i}}{h_{2}}-\frac{u_{i}-u_{i-1}}{h_{1}}\right)
$$

In addition, the following equation is used for grid stretching near the wall region,

where,

$$
y(i)=\frac{y^{+}(i)}{R e_{\tau}}
$$

$$
y^{+}(i)=\operatorname{Exp}\left(\log \left[y^{+}(1)\right]+(i-1)\left(\frac{\log \left[R e_{\tau} / y^{+}(1)\right]}{N-1}\right)\right.
$$

For all the simulations, first grid point $\left(y^{+}\right)$adjacent to the wall is considered as 0.025 (presented in Table B.1). Moreover, the tri-diagonal matrix algorithm (TDMA) method is used to solve the linear values of partial differential equations. Moreover, iterative method has been used to solve the governing equations. In simulations, for the primary guess the following initial velocity profiles are considered in the laminar (near the wall) and the turbulent (far off the wall) region,

$$
u^{+}=y^{+} \quad \text { for } y^{+}<10
$$

and

$$
u^{+}=\frac{1}{\kappa} \ln \left(y^{+}\right)+B \quad \text { for } y^{+} \geq 10
$$

where, $\kappa=0.41$ is the von Karman constant and $B$ is the integration constant which is considered 5.2.

Table B.1. Considered $y^{+}$values for Cebeci-Smith and $k-\varepsilon$ model

\begin{tabular}{|c|c|}
\hline RANS Models & $\left(\boldsymbol{y}^{+}\right)$values \\
\hline Cebeci-Smith & 0.025 \\
\hline$k-\varepsilon$ model & 0.025 \\
\hline
\end{tabular}

\section{B.4 Cebeci-Smith model}

To account for the eddy viscosity in one-dimensional turbulent flow inside the pipe, the CebeciSmith (Cebeci and Smith 1974), which is also known as the zero equation turbulence model, has 
been implemented in the RANS code accordingly. During calculation of eddy viscosity it divides the boundary layer in two different zones (Wilcox 1993).

$$
v_{t}= \begin{cases}v_{t_{\text {inner }}} & \text { for } y \leq y_{\text {crossover }} \\ v_{t_{\text {outer }}} & \text { for } y \leq y_{\text {crossover }}\end{cases}
$$

Here, $y_{\text {crossover }}$ is the smallest distance which separates two layers of the boundary layer (inner and outer) where $v_{t_{\text {inner }}}$ is equal to $v_{t_{\text {outer }}}$. Additionally, $v_{t_{\text {inner }}}$ represents the turbulent viscosity near the wall and $v_{t_{\text {outer }}}$ computes the outer boundary layer and can be expressed as follows,

$$
v_{t_{\text {inner }}}=l_{\text {mix }}^{2}\left[\left(\frac{\partial u}{\partial y}\right)^{2}+\left(\frac{\partial v}{\partial x}\right)^{2}\right]^{1 / 2}
$$

where,

$$
l_{m i x}=\kappa y\left[1-e^{-y^{+} / A^{+}}\right]
$$

Here, $\kappa=0.41$, and $A^{+}=26\left[1+y(d p / d x) / \rho u_{\tau}^{2}\right]^{-1 / 2}$ are closure coefficients respectively. And

$$
v_{t_{\text {outer }}}=\alpha u_{e} \delta_{v}^{*} F_{K l e b}(y ; \delta)
$$

where, $\alpha=0.0168$ is closure coefficient and $u_{e}$ is boundary layer edge velocity. The term $F_{K l e b}$ is the Klebanoff intermittency function and $\delta_{v}^{*}$ is the velocity thickness that can be defined as,

And

$$
F_{K l e b}(y ; \delta)=\left[1+5.5\left(\frac{y}{\delta}\right)^{6}\right]^{-1}
$$

$$
\delta_{v}^{*}=\int_{0}^{\delta}\left(1-u / u_{e}\right) d y
$$

all these closure coefficients and additional terminology are incorporated in the code properly to compute the eddy viscosity using the Cebeci-Smith zero equation turbulence model. Later, the numerical results obtained are compared to the DNS results. 


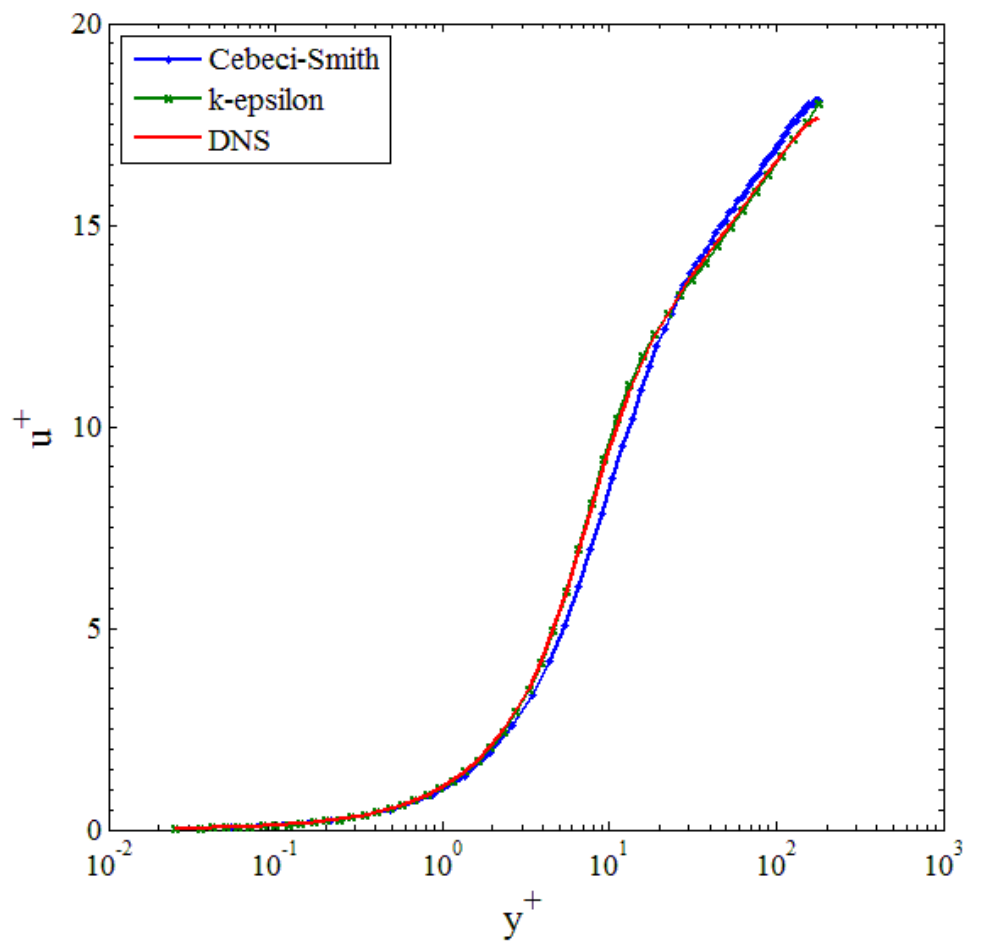

Figure B.3. Dimensionless velocity profile at Reynolds number $\left(R e_{\tau}=180\right)$

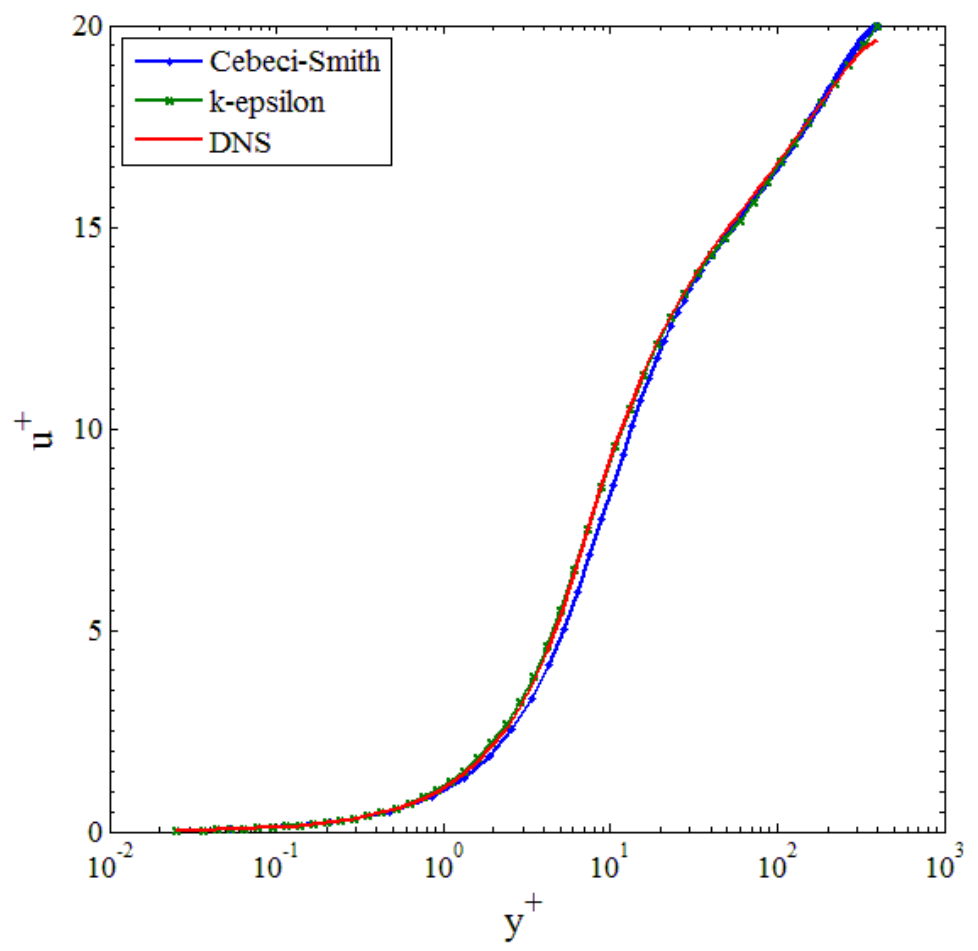

Figure B.4. Dimensionless velocity profile at Reynolds number $\left(R e_{\tau}=395\right)$ 


\section{B.5 $\boldsymbol{k}-\boldsymbol{\varepsilon}$ model}

For simulations of fully developed turbulent channel flow, turbulence is also accounted for by using the $k-\varepsilon$ model. The $k-\varepsilon$ eddy model is the widely used two-equations turbulence model. The detailed description $k-\varepsilon$ model has been discussed in the Reynolds averaged Navier-Stokes (RANS) section of Chapter 1. The following equation has been used to account for the eddy viscosity,

$$
v_{T}=\frac{C_{\mu} k^{2}}{\varepsilon}
$$

To compute the turbulent kinetic energy $(k)$ and the dissipation $(\varepsilon)$, the following equations have been used,

$$
\begin{gathered}
\frac{\partial k}{\partial t}+\bar{u}_{j} \frac{\partial k}{\partial x_{j}}=-\overline{u_{\imath}^{\prime} u_{\jmath}^{\prime}} \frac{\partial \bar{u}_{\imath}}{\partial x_{j}}-\varepsilon+\frac{\partial}{\partial x_{j}}\left[\left(v+v_{T} / \sigma_{k}\right) \frac{\partial k}{\partial x_{j}}\right] \\
\frac{\partial \varepsilon}{\partial t}+\bar{u}_{j} \frac{\partial \varepsilon}{\partial x_{j}}=-C_{\varepsilon 1} \frac{\varepsilon}{k} \overline{u_{\imath}^{\prime} u_{\jmath}^{\prime}} \frac{\partial \bar{u}_{\imath}}{\partial x_{j}}-C_{\varepsilon 2} \frac{\varepsilon^{2}}{k}+\frac{\partial}{\partial x_{j}}\left[\left(v+v_{T} / \sigma_{\varepsilon}\right) \frac{\partial \varepsilon}{\partial x_{j}}\right]
\end{gathered}
$$

Here, $C_{\varepsilon 1}=1.44, C_{\varepsilon 2}=1.92, C_{\mu}=0.09, \sigma_{k}=1.0, \sigma_{\varepsilon}=1.3$ are closure coefficients and constants that are implemented in the RANS code. Moreover, the numerical solution obtained using the $k-\varepsilon$ model has been compared to the existing DNS results for the taken Reynolds numbers to check the accuracy of the model.

\section{B.6 Results and discussion}

For one-dimensional turbulent pipe flow, the dimensionless velocity profile $\left(u^{+}\right)$is compared to normalized distance from the wall $\left(y^{+}\right)$for Reynolds numbers $\left(R e_{\tau}\right) 180$ and 395 based on wall friction velocity. Obtained numerical results of pipe flow from Cebeci-Smith and $k-\varepsilon$ models are compared to the DNS results of Kim et al. (1987) for $R e_{\tau}=180$ and Moser et al. (1998) for $R e_{\tau}=395$. Here, Figure B.3 shows a comparison of the Cebeci-Smith and the $k-\varepsilon$ models to 
the DNS results of $R e_{\tau}=180$. From the comparison it appears that $k-\varepsilon$ shows very good agreement with the DNS data whereas the Cebeci-Smith model shows a significant variation. In Figure B.4, the numerical solution of the Cebeci-Smith and $k-\varepsilon$ models are compared to DNS data for Reynolds number $R e_{\tau}=395$. Similar to the comparison of $R e_{\tau}=180$, it appears that at a high Reynolds, number $R e_{\tau}=395$, once again the $k-\varepsilon$ model shows very good agreement to the DNS data compared to the Cebeci-Smith model. In both Reynolds number cases CebeciSmith shows marginal deviation compared to DNS data.

The most probable reason is that the Cebeci-Smith model is an algebraic model which uses mixing length theory to compute eddy viscosity. Moreover, it does not solve any partial differential equations (PDE's) to correlate the eddy viscosity to lengthscales of the mean flow. For this reason, these types of algebraic models are defined as 'incomplete' eddy models for accounting for turbulent viscosity. Hence, algebraic models are not used for turbulence modelling of practical applications. On the other hand, the $k-\varepsilon$ model solves two additional PDE's for turbulent kinetic energy $(k)$ and the dissipation $(\varepsilon)$ to account for turbulent viscosity and correlates the obtained eddy viscosity to the mean flows. These features make the $k-\varepsilon$ model more powerful to account for the turbulence in many practical applications where secondary fluid flow motions such as vortices and flow separation take place. Another important feature of the $k-\varepsilon$ model is that it is also able to predict the transition from laminar to turbulent flow. Moreover, due to all of these features, the $k-\varepsilon$ model is capable of predicting turbulent flow relatively more accurately compared to the algebraic or zero equation turbulence models.

\section{B.7 Conclusions}

The one-dimensional turbulent pipe flow case study focuses on the importance of eddy viscosity models to predict turbulent flows accurately. This study also shows the accuracy of the second- 
order numerical scheme which has been used to solve the Navier-Stokes equations. Moreover, this numerical study gives emphasis on grid generation and their contribution to obtain numerical solutions of turbulent flow with sufficient accuracy. Grids are stretched along the radial direction using exponential grid generation processes. Near the wall, grids are kept sufficiently small to capture the wall effect on turbulence properly. The $y^{+}$value adjacent to the wall is considered as 0.025 for both RANS models. From an analysis of the result it appears that the assigned $y^{+}$value is sufficiently small to capture the turbulence near the wall properly. Moreover, the numerical results obtained using the Cebeci-Smith and the $k-\varepsilon$ models at different Reynolds number are compared to the DNS results. From the comparison, it appears that compared to Cebeci-Smith model, $k-\varepsilon$ model is in very good agreement with the DNS results. Moreover, outcomes of this numerical study demonstrate that the second-order numerical code (default version of FDS uses a second-order numerical scheme to solve the governing equations) is capable of reproducing the DNS results with sufficient accuracy. 


\section{Appendix C}

\section{Relation between grid and $U_{e}$}

To maintain the flow continuity, higher boundary layer edge velocity is predicted for coarser grids compared to the simulations where finer grid is used (refer to Chapter 3 velocity profile Figures 3.2 and 3.3). The following velocity contour diagrams with coarse and fine grids (refer to Table 3.1) at location $x / h=4$ is presented here to demonstrate this rationale,

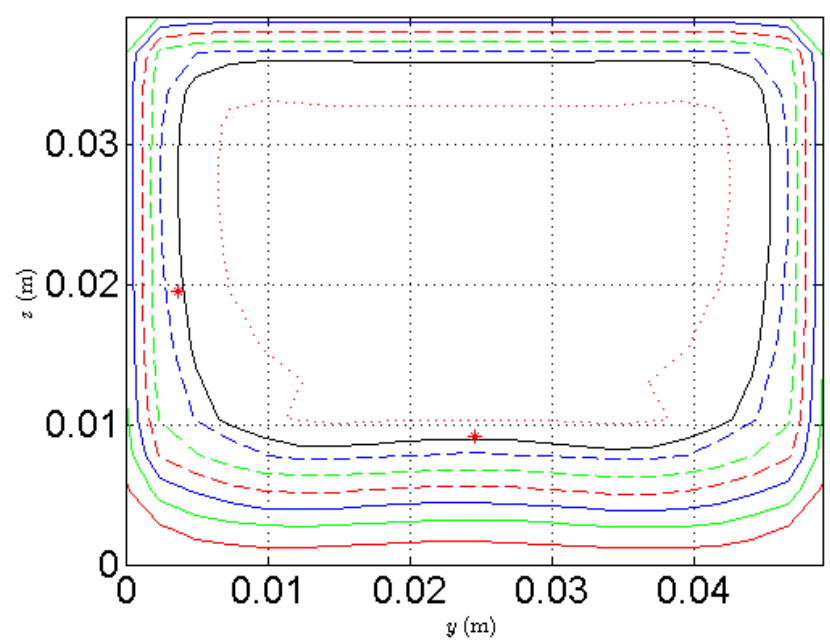

(a) Coarse grid

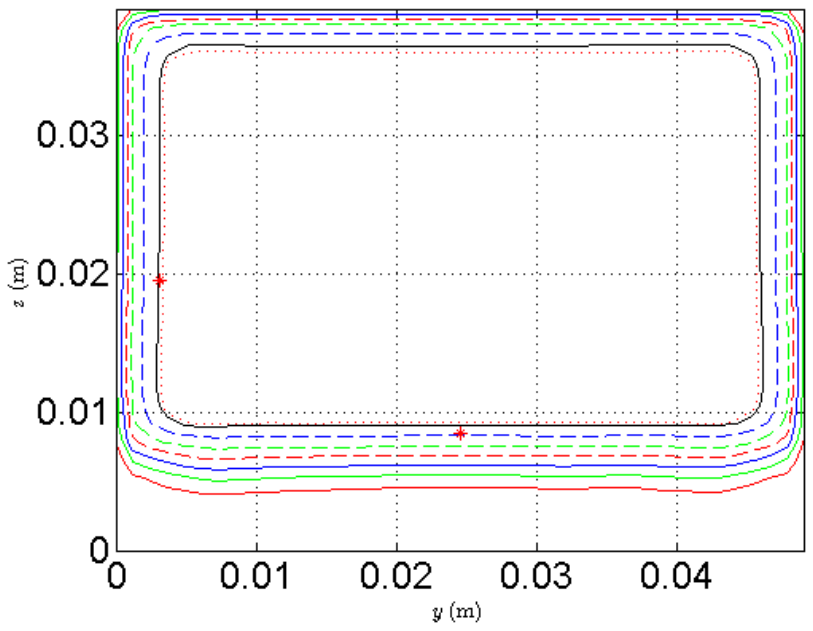

(b) Fine grid

Figure C.1. Velocity contour plots at location $x / h=4$ where black lines represent predicted $0.9 U_{e}$ contours for coarse and fine grids 